\title{
Radiotracers for Examining Biological Functions of Plants and
} Microbes

\author{
A dissertation presented to the \\ Faculty of the Chemistry Department at the \\ University of Missouri-Columbia \\ In Partial Fulfillment \\ of the Requirements for the Degree \\ Doctor of Philosophy \\ by \\ Alexandra Bauer Housh \\ Dr. Richard A. Ferrieri, Dissertation Supervisor \\ May 2021
}


The undersigned, appointed by the dean of the Graduate School, have examined the dissertation entitled

\section{Radiotracers for Examining Biological Functions of Plants and Microbes}

Presented by Alexandra Bauer Housh

a candidate for the degree of Doctor of Philosophy

and hereby certify that, in their opinion, it is worthy of acceptance.

Professor Richard Ferrieri

Professor Silvia Jurisson

Professor Michael Greenlief

Professor Gary Stacey 


\section{DEDICATION}

To my mentors and professors

past and present

who empowered me and shaped me

into the scientist I am today.

This is for you. 


\section{ACKNOWLEDGEMENTS}

I must first acknowledge my advisor, Dr. Richard Ferrieri. Learning the ins and outs of the field from you has been the greatest pleasure. More than that, you showed me what it means to love what you do. Truly, you are the best mentor I have ever had. I am thankful for our entire research group, past and present, which often felt like family and whom I will always keep in my heart as friends. I would also like to thank Drs. Jurisson, Greenlief, and Stacey for being a part of my doctoral committee and shaping my graduate school experience. Your input has been invaluable.

Behind every successful woman, there is the family that shaped her. To my parents- I would not be here today, or be who I am today, without you. A very special acknowledgement goes out to my siblings, Robby, Bethany and Dylan- whose footsteps I have always walked in and who taught me resilience in the face of challenges. To everyone; aunts, uncles, cousins, grandparents, siblings, nieces and nephews- all of my achievements are, in part, a result of your support, love, example, and sacrifice. When I think of an event in my life worth celebrating, I want most to celebrate with you.

Thank you to my friends, who have become like family. There are too many to name, and not enough words to express how important you all have been to my morale throughout this graduate school process. There were days I felt lost, but the encouraging texts, letters, gifts, hugs, board game marathons, movie nights, study dates, and delicious food cooked together helped me find my way again. I did it, but I did not have to do it alone.

Last, but certainly not least, I want to acknowledge my husband, Kurt. You have been my dearest friend, greatest comfort, and main inspiration throughout my graduate school experience. You make me want to be the best version of myself every single day. Earning our doctorates together is something of which I am immeasurably proud. Thank you for always believing in me. I cannot wait to see what we accomplish next. 


\section{Table of Contents}

Acknowledgements

List of Figures

$\begin{array}{ll}\text { List of Tables } & \mathbf{x}\end{array}$

List of Equations $\quad$ xi

$\begin{array}{lll}\text { Abstract } & \text { xii }\end{array}$

Chapter One: Nuclear and Mass Spectrometry Based Technologies to Examine Plant Nutrition and Nutrient Acquisition

1.1 Importance of Understanding Plants - Origins of Radiotracers in Plant Science $\quad 1$

1.2 Radioisotope Tracers: Evaluation of Nuclear Technologies 3

1.3 Plant Nutrient Needs and Plant Growth Promoting Bacteria as a Nutrition-

$\begin{array}{ll}\text { Enhancement Approach } & 7\end{array}$

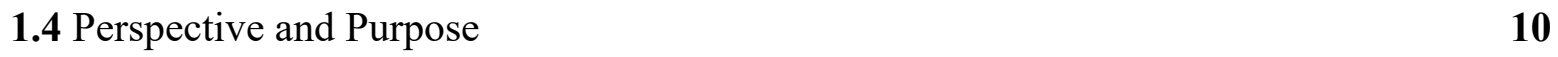

$\begin{array}{ll}1.5 \text { Abbreviations } & 11\end{array}$

$\begin{array}{ll}\text { References } & 12\end{array}$

Chapter Two: Assessment of a $\left[{ }^{18}\right.$ F]-4-Fluorophenylboronic Acid Radiotracer for Imaging Boron in Maize

$\begin{array}{ll}\text { 2.1 Preface } & 17\end{array}$

$\begin{array}{ll}2.2 \text { Introduction } & 17\end{array}$

$\begin{array}{lr}2.3 \text { Results } & \mathbf{2 0}\end{array}$

$\begin{array}{ll}2.4 \text { Discussion } & 31\end{array}$

2.5 Materials and Methods $\quad 38$

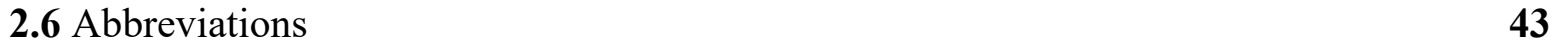

References $\quad 44$

Chapter Three: Functional mutants of Azospirillum brasilense elicit beneficial physiological and metabolic responses in Zea mays contributing to increased host iron assimilation while boosting crop yield and seed iron and ferritin content.

3.1 Preface 
3.3 Results and Discussion $\quad \mathbf{5 2}$

3.4 Materials and Methods $\quad \mathbf{7 6}$

$\begin{array}{ll}3.5 \text { Abbreviations } & 93\end{array}$

$\begin{array}{ll}\text { References } & 94\end{array}$

Chapter Four: Plant Growth Promoting Bacteria Improve Maize Zinc Assimilation: An Examination of the Mechanisms of Action using Functional Mutants of Azospirillum brasilense

$\begin{array}{ll}\text { 4.1 Preface } & 101\end{array}$

$\begin{array}{ll}4.2 \text { Introduction } & 101\end{array}$

$\begin{array}{ll}\text { 4.3 Results and Discussion } & 103\end{array}$

$\begin{array}{ll}4.4 \text { Conclusion } & 108\end{array}$

$\begin{array}{ll}\text { 4.5 Materials and Methods } & 108\end{array}$

$\begin{array}{ll}\text { 4.6 Abbreviations } & 112\end{array}$

$\begin{array}{ll}\text { References } & 113\end{array}$

Chapter Five: Examining Effects of Rhizobacteria in Ameliorating Abiotic Crop Stresses using Carbon-11 Radiotracing

5.1 Preface $\quad 116$

5.2 Introduction $\quad 116$

$\begin{array}{ll}5.3 \text { Results } & 118\end{array}$

5.4 Discussion $\quad 126$

$\begin{array}{lr}5.5 \text { Conclusion } & 130\end{array}$

$\begin{array}{ll}\text { 5.6 Materials and Methods } & 130\end{array}$

$\begin{array}{ll}5.7 \text { Abbreviations } & 137\end{array}$

$\begin{array}{ll}\text { References } & 138\end{array}$ 
Chapter Six: A Guiding Light to Sustaining Life Functions Belowground: Light effects on biological nitrogen fixation, carbon dioxide assimilation, and iron uptake in functional mutants of Azospirillum brasilense.

$\begin{array}{ll}\text { 6.1 Introduction } & 144\end{array}$

6.2 Results and Discussion 149

$\begin{array}{ll}\text { 6.3 Conclusion } & 163\end{array}$

$\begin{array}{ll}\text { 6.4 Materials and Methods } & 165\end{array}$

$\begin{array}{ll}\text { 6.5 Abbreviations } & 173\end{array}$

$\begin{array}{ll}\text { References } & 174\end{array}$

Chapter Seven: Concluding Remarks: Global Concerns with Future Sustainable Agriculture and Future Use of Radiotracers in the Real-World

7.1 A Recap of Challenges Facing Sustainable Agriculture $\quad 178$

7.2 How Far We Have Come 179

7.3 Rhizospheric Microorganisms as a Community- Not Isolates 180

$\begin{array}{ll}\text { 7.4 Real-World Radiotracing } & 181\end{array}$

$\begin{array}{ll}\text { References } & 183\end{array}$

Appendix: Correlating Bacterial Biological Function to Plant Carbon Allocation and Exudation

$\begin{array}{lr}\text { A.1 Preface } & 184\end{array}$

$\begin{array}{ll}\text { A.2 Introduction } & 184\end{array}$

$\begin{array}{ll}\text { A.3 Materials and Methods } & 185\end{array}$

$\begin{array}{lr}\text { A.4 Results } & 193\end{array}$

$\begin{array}{ll}\text { References } & 194\end{array}$

$\begin{array}{ll}\text { Vita } & 195\end{array}$ 


\section{LIST OF FIGURES}

\section{Chapter One Figures}

1.3.1. Comparison of auxin production capacities of Azospirillum brailense functional mutants

\section{Chapter Two Figures}

2.3.1. Synthesis and Administration of a $\left[{ }^{18} \mathrm{~F}\right] \mathrm{FPBA}$ radiotracer to maize seedlings $\quad \mathbf{2 1}$

2.3.2. Original images for composite image in Figure 2.3.1 B

2.3.3. Isolated root images, showing localization of $\left[{ }^{18} \mathrm{~F}\right] \mathrm{FPBA},\left[{ }^{18} \mathrm{~F}\right] \mathrm{FDG}$ and

$\left[{ }^{11} \mathrm{C}\right]$ photoassimilates in roots

2.3.4. Phenotypes of maize root growth (B73 inbred) germinating in different concentrations of PBA and FPBA

2.3.5. Phenotypes of maize $B 73$ root growth germinating in different concentrations of BA

2.3.6. Percentages of $B 73$ maize seedlings with lateral roots five days after germination

2.3.7. Phenotypes of seedlings resulting from PBA and FPBA treated Arabidopsis thaliana siliques

2.3.8. Mimicking of B deficiency symptoms by PBA/FPBA

2.3.9. Analysis of primary root lengths of $B 73$ maize seedlings treated with water, BA, FPBA, and PBA

2.3.10. Primary root length relative to water control of WT, rte/+, and rte/rte maize seedlings that were germinated in different concentrations of PBA

2.3.11. ICP-MS analysis of different elemental contents in roots of five days old maize seedlings (B73 inbred), that were germinated in FPBA or water

\section{Chapter Three Figures}

3.2.1. Mechanisms for higher plant assimilation of iron from soil

3.3.1. Radioactive Fe-59 reveals features for A. brasilense promotion of host assimilation and whole-plant transport of ferrous iron $\left(\mathrm{Fe}^{2+}\right)$ and ferric iron $\left(\mathrm{Fe}^{3+}\right)$

3.3.2. Drop plate assay analysis on the extent of bacteria colonization of maize roots 
3.3.3. Radiographic imaging of plant tissues reveal different spatial patterning of radioactive ${ }^{59} \mathrm{Fe}$ as ferric $\left(\mathrm{Fe}^{3+}\right)$ and ferrous $\left(\mathrm{Fe}^{2+}\right)$ ionic forms

3.3.4. LA-ICP-MS and TEM reveal spatial patterning of Fe-56 in plant tissues that correlates with root cellular morphological changes due to beneficial microbes

3.3.5. Carbon-11 aids in mapping maize physiological and metabolic responses to bacteria inoculation

3.3.6. Visual assay of root $\mathrm{pH}$ in 3-day old maize seedlings reveals a higher level of acidic $\mathrm{pH}$ surrounding HM053 and FP10 inoculated roots

3.3.7. Shared metabolic branch point in the biosynthesis of Auxin and DIMBOA

3.3.8. In vitro chemotaxis assays to examine effects of DIMBOA on Bacteria growth

3.3.9. Plant growth rates measured as plant height and stem diameter over time

3.3.10. Leaf chlorophyll content and leaf thickness as a function of treatment type

3.3.11. Effect of treatment on corn seed yield. Seed yield was quantified as number of kernels per cob

3.3.12. Seed iron content as total iron, ferric iron, and ferrous iron as a function of treatment type

3.3.13. Seed iron-ferritin content as a function of treatment type

3.4.1. Maize plants raised for ${ }^{59} \mathrm{Fe}$ studies were grown aeroponically in a commercial growth chamber

3.4.2. Maize plants raised in Turface ${ }^{\mathrm{TM}}$ for ${ }^{11} \mathrm{C}$ radiotracer studies

3.4.3. Outdoor potted plant studies

\section{Chapter Four Figures}

4.3.1. Dynamic Zn-65 transport over 3-hours of acquisition

4.3.2 $\mathrm{NaI}(\mathrm{Tl})$ gamma detection of plant tissues exhibit differences in uptake and allocation of $\mathrm{Zn}-65$ associated with different functional mutants of $A$. brasilense 4.3.3. Principal component analysis correlates $\mathrm{Zn}-65$ uptake and root-shoot allocation

4.3.4. Radiographic images of maize plants after exposure to $\mathrm{Zn}-65$ as a function of inoculation with ipdC, FP10 and HM053 bacteria 
4.3.5. Kernel $\mathrm{Zn}$ content measured using ion chromatography

\section{Chapter Five Figures}

5.3.1. Root Characteristics of Maize under Abiotic Stress

5.3.2. Carbon-11 aids in mapping maize physiological responses to applied abiotic stresses of salinity and nitrogen limitation and to the introduction of microbial inoculant onto these stresses

5.3.3. Carbon-11 aids in mapping changes in maize carbon metabolism as a function of applied abiotic stresses of salinity and nitrogen limitation and to the introduction of microbial inoculant onto these stresses

5.3.4. Tissue starch levels are presented for shoots and root tissues as a function of salinity stress

5.3.5. ICP-MS data shows levels of $\mathrm{K}^{+}$and $\mathrm{Ca}^{2+}$

\section{Chapter Six Figures}

6.2.1. Light Piping Setup and Evidence of Light Piping in Maize

6.2.2. ARA of $A$. brasilense inoculated maize roots as a function of white light and total darkness

6.2.3. ARA of $A$. brasilense inoculated maize roots as a function of blue and red light exposure

6.2.4. ${ }^{59} \mathrm{Fe}^{2+/ 3+}$ Radiotracer uptake as a percentage of total tracer administered over time as a function of light status and bacteria biological function

6.2.5. Principal component analysis of ${ }^{59} \mathrm{Fe}^{2+/ 3+}$ radiotracer uptake data over time as a function of light status and bacteria biological function

6.2.6. Percent Assimilation of $\left[{ }^{11} \mathrm{C}\right] \mathrm{CO}_{2}$ in bacteria liquid cultures in darkness and exposed to white lights across morning and afternoon

6.2.7. $\left[{ }^{11} \mathrm{C}\right] \mathrm{CO}_{2}$ Metabolism Charts for the HM053 functional mutant of $A$. brasilense

6.2.8. $\left[{ }^{11} \mathrm{C}\right] \mathrm{CO}_{2}$ Metabolism Charts for the ipdC functional mutant of $A$. brasilense 
6.2.9. $\left[{ }^{11} \mathrm{C}\right] \mathrm{CO}_{2}$ Metabolism Charts for the FP10 functional mutant of $A$. brasilense

6.4.1. Image Intensifier for capturing light piping in maize

6.4.2. Experimental set up for acetylene reduction assay

6.4.3. Experimental set up for acetylene reduction assay root incubation chamber and sampling port

6.4.4. Experimental Set-up for $\left[{ }^{11} \mathrm{C}\right] \mathrm{CO}_{2}$ assimilation studies

171

\section{Appendix Figures}

A.3.1. Switchgrass in rhizobox setup

A.3.2. Localization of high colonization of roots by RAM10 H. seropedicae by

fluorescence imaging

A.3.3. $\left[{ }^{13} \mathrm{C}\right] \mathrm{CO}_{2}$ incubation chamber setup for switchgrass plants

A.3.4. Sample preparation for LAIRMS analysis

190

A.3.5. Root mapping of ablations and data collection for analysis

192 


\section{LIST OF TABLES}

\section{Chapter One Tables}

1.3.1. Essential Nutrients for Plants

\section{Chapter Two Tables}

2.3.1. Reactions conditions investigating solvents for maximum yield

2.3.2. Reaction conditions investigating reaction time and temperature for maximum yield $\mathbf{2 3}$ 


\section{LIST OF EQUATIONS}

Chapter Three Equations

$\begin{array}{lr}\text { 3.4.1. Calculating Specific Activity } & \mathbf{8 3}\end{array}$

$\begin{array}{ll}\text { 3.4.2. Radiation Decay Equation } & \mathbf{8 3}\end{array}$ 


\title{
Radiotracers for Investigating Biological Functions of Plants and Microbes
}

\author{
Alexandra Bauer Housh \\ Dr. Richard A. Ferrieri, Dissertation Advisor \\ ABSTRACT
}

Tracers are used for qualitative and quantitative investigation of a system. Radiotracers have a radionuclide to observe chemical or biological processes by detection of the radionuclide's decay energy. They are non-disruptive and non-destructive to living systems and can be quantified, imaged, and measured in real time, adding value. This work focuses on radiochemistry and radiotracer techniques to understand maize uptake and localization of micronutrients and the impact of Azospirillum brasilense microbial interactions on these processes. Further, it explored how such interactions can influence stress responses in maize. Finally, it examined how the natural biological functions of $A$. brasilense bacteria respond to light stimulus conducted through the plant tissues.

In this dissertation, the efficacy of using 4-fluorophenylboronic acid (FPBA) as a boton (B) imaging agent, which is a derivative of the B deficiency mimic phenylboronic acid (PBA), was explored. It is shown that radioactively labelled $\left[{ }^{18} \mathrm{~F}\right] \mathrm{FPBA}\left(\mathrm{t}_{1 / 2}=110 \mathrm{~m}\right)$ accumulates at the root tip, the root elongation zone and at lateral root initiation sites in maize roots, and also translocates to the shoot where it accumulates along leaf edges. This is the first time a radiotracer has been utilized to image B in plant systems. Nutritional iron $(\mathrm{Fe})$ content was explored in Azospirillum brasilense associated maize. ${ }^{59} \mathrm{Fe}\left(\mathrm{t}_{1} / 2=44.5 \mathrm{~d}\right)$ was used to trace iron uptake kinetics and allocation to leaf. In the presence of functional mutants of this bacteria, iron uptake and allocation to leaf was enhanced in maize seedlings. Maize were grown to maturity and plants associated with the bacteria had greater crop yield (kernels $\left.\mathrm{cob}^{-1}\right)$ and enhanced iron and protein ferritin- the bioavailable form of iron to humans- seed content. Similar studies were completed using zinc $\left({ }^{65} \mathrm{Zn}, \mathrm{t}_{1}=244 \mathrm{~d}\right)$, where it was noted that the presence of the low-auxin producing and 
nitrogen-fixing bacteria strain, ipdC, enhanced zinc uptake but had no enhancement effect on allocation or zinc seed filling. Carbon metabolism in response to stresses and microbial interaction was also investigated in maize with $\left[{ }^{11} \mathrm{C}\right] \mathrm{CO}_{2}\left(\mathrm{t}_{1} / 2=20.4 \mathrm{~m}\right)$ radiotracer. In association with $A$. brasilense, maize fixed more carbon dioxide, allocated more ${ }^{11} \mathrm{C}$-photosynthates to the roots, and produced more ${ }^{11} \mathrm{C}$-exudates than control maize. Metabolic differences were studied via radio-HPLC and radio-TLC to reveal association enhanced ${ }^{11} \mathrm{C}$ flow into hydrophobic structural components and amino acids. When nitrogen stressed, non-inoculated maize exhibited a decrease in carbon dioxide fixation, root allocation of ${ }^{11} \mathrm{C}$-photosynthates, and decreased ${ }^{11} \mathrm{C}$ exudation compared to control maize. They also saw increased ${ }^{11} \mathrm{C}$ flow into hydrophobic structural components and sugars. When inoculated with $A$. brasilense and subjected to nitrogen stress, the same enhancements occurred- but fixation, allocation, and exudation recovered to near control maize levels, suggesting these bacteria ameliorate some abiotic stresses. Finally, ${ }^{59} \mathrm{Fe}$ and $\left[{ }^{11} \mathrm{C}\right] \mathrm{CO}_{2}$ radiotracers were applied to the functional mutants of $A$. brasilense to uncover how various biological functions were impacted by light exposure. First, light transmittance from shoot to root tissues, called light piping, in maize was shown using a DSLR camera and image intensifier. Studies showed the functional mutants with biological nitrogen fixation (BNF) capacity had enhanced assimilation of ${ }^{59} \mathrm{Fe}$ when exposed to light relative to dark treatments and greater activity of the nitrogenase enzyme as measured by acetylene reduction assay in light, with a greater response noted for red than blue light wavelengths. Carbon assimilation as $\left[{ }^{11} \mathrm{C}\right] \mathrm{CO}_{2}$ and subsequent metabolism in these bacteria were also impacted by light stimulus. 


\section{Chapter One: Nuclear Based Technologies to Examine Plant Nutrition and Nutrient}

\section{Acquisition}

\subsection{Importance of Understanding Plants - Origins of Radioisotope Tracers in Plant Science}

Plants play a vital role in the world and interact with both living and non-living things every day. Humans depend on plants for various purposes including food, shelter, medicine, fuel, and fibers ${ }^{1}$. Beyond that, plants can function to reduce erosion, mitigate pollution, and contribute to nutrient-cycling, which a plethora of organisms, from bacteria to humankind, rely on to survive. Because their functions are so important, the study of plants is a crucial endeavor to the advancement, and even survival, of our species and to maintaining biodiversity of others.

To further that endeavor, isotope tracers have been used as a unique and powerful method for gaining insight into the biological and physical functions of plants. They can be used to follow metabolites from initial plant uptake via roots or shoots through every step in a plant's metabolic processes and can even help researchers examine a plant's nutrient exchange in the presence of other organisms in their complex ecosystem. Some of the more common tracers used in plant research are isotopes of carbon. In total, there are fourteen isotopes of carbon, two of which are stable and twelve of which are radioisotopes. Only a subset are useful in examining biological processes based on their half-lives and availability, predominantly ${ }^{11} \mathrm{C},{ }^{12} \mathrm{C},{ }^{13} \mathrm{C}$, and ${ }^{14} \mathrm{C}$.

In the past, radioactive isotopes of carbon have provided enormous insight into how plants use their carbon resources. The earliest experiments on the pathways of carbon in photosynthetic and heterotrophic metabolism using radioactive carbon, ${ }^{11} \mathrm{C}$, as a tracer were performed by Martin Kamen, Samuel Ruben and their colleagues in $1938^{2}$. However, the short half-life of ${ }^{11} \mathrm{C}$ $\left(\mathrm{t} \frac{1}{2} 20.4 \mathrm{~m}\right)$ posed severe limitations on identification of metabolic intermediates with the analytical technology available at the time. This limitation was the major stimulus for using a 
radioactive carbon isotope of longer half-life, ${ }^{14} \mathrm{C}(\mathrm{t} t / 25280 \mathrm{y})^{3}$. In later studies, there was renewed interest and use of ${ }^{11} \mathrm{C}$ in plant studies because of the ease in measuring gamma radiation non-destructively in plant tissues - something that ${ }^{14} \mathrm{C}$ could not provide. Indeed, numerous investigations can be found where $\left[{ }^{11} \mathrm{C}\right] \mathrm{CO}_{2}$ was leveraged to measure ${ }^{11} \mathrm{C}$ photosynthate transport within intact plants, which provided enormous insight into source-sink relations (for a review see reference 4$)^{4}$. However, with the availability of modern radioanalytical tools, there has been another resurgence in the use of ${ }^{11} \mathrm{C}$ radiotracing to examine plant metabolic regulation. Applications here have included studies on plant-pest interactions both aboveground ${ }^{5-10}$ and belowground ${ }^{11,12}$, studies on plant starch regulation and carbon mobilization ${ }^{13}$, hormone signaling ${ }^{14,15}$, and more recently in studies on plant-microbe interactions ${ }^{16-19}$.

While it is one of the most utilized radioisotope tracers in plant research, carbon is not the only element useful in understanding plant metabolism and plant interactions with the environment and organisms around it. One of the first radiotracers used in plants was a radioisotope of lead $\left({ }^{212} \mathrm{~Pb}\right)$ by George de Hevesy in 1923 to investigate broad bean uptake and allocation of $\mathrm{Pb}^{20}$. Indeed, various radiotracers have been utilized to investigate plant processes such as ${ }^{22} \mathrm{Na}$ to investigate dynamic salt uptake in Setaria veridis ${ }^{21},{ }^{59} \mathrm{Fe}$ to study iron remobilization efficiency toward nitrogen and iron deficiency resistance in wheat ${ }^{22}$, and even ${ }^{18} \mathrm{~F}$ labeled fluorodeoxyglucose $\left(\left[{ }^{18} \mathrm{~F}\right] \mathrm{FDG}\right)$, originally synthesized to study living human brain glucose metabolism in $1976^{23}$, to investigate and image photoassimilate translocation in various plant species ${ }^{24}$. More elements and tracers will be discussed later throughout this document. It is important to emphasize that understanding plant metabolism and how and when it is altered is 
vital in modern agriculture, i.e., plant biotechnology to improve crop bioenergy efficiency, crop calorie and nutritional production, as well as medicinal/materials utility.

\subsection{Radioisotope Tracers: Evaluation of Nuclear Technologies}

Radiotracers are compounds containing in their structure at least one radionuclide for the purpose of observing physical, chemical, or biological processes by detection of the radionuclide's decay energy ${ }^{25}$. In an ideal radiotracer, a radioisotope replaces a stable isotope of the same element. This substitution works because, theoretically, all isotopes of a given element behave identically in terms of their physical and chemical characteristics. Using non-radioactive chemical methods of quantification, nano- to microgram amounts of metabolite are generally needed to elicit a response. Radiotracers allow detection with approximately $10^{5}$ smaller quantities because each detectable disintegration corresponds to the decay of just a single atom ${ }^{25}$. Thus, it becomes possible to detect metabolites occurring at very low concentrations that are challenging to detect via other chemical methods. It is also then possible to account for each atom of radioisotope introduced into a system, which is challenging with stable isotope experiments.

The use of radiotracers requires basic assumptions be made including; (1) radioactivity administered does not alter, physically or chemically, the system investigated; (2) the amount of radiotracer administered does not change the natural physiological concentration of unlabeled metabolite; (3) only the radionuclide itself is traced, which may not reflect the original chemical form when first introduced to the system; and (4) the radiotracer behaves identically chemically and physically, as the unlabeled compound ${ }^{25}$. A caveat to the fourth assumption is the kinetic isotope effect. In cases where the radioisotope being used for the radiotracer is different enough in mass to the stable, natural isotope there can be differences in chemical and physical processes 
such as diffusion, dissolution, and enzyme-mediated steps ${ }^{26}$. This is observed for isotopes of carbon, in which the difference is diffusion speed, dissolution, and even carboxylation during the photosynthesis steps cause differences in ${ }^{13} \mathrm{C} /{ }^{12} \mathrm{C}$ ratios across various plant tissues. In fact, ${ }^{13} \mathrm{C} /{ }^{12} \mathrm{C}$ measurements are often taken in plant tissues to classify them by their photosynthetic pathways $^{26}$. The kinetic isotope effect is not only noted in lighter elements such as carbon; it is also noted in heavier micronutrients in plants, like zinc, where the ${ }^{66} \mathrm{Zn} /{ }^{64} \mathrm{Zn}$ ratio differs between root and shoot tissues as well as from the soil in which plants are found ${ }^{27}$. Such isotope effects are also observed for radioisotopes of elements.

The radiotracers used in this dissertation research are predominantly positron emitters, with some beta and gamma emitters as well. In positron emission, the nucleus decays by emission of a positron, or anti-electron ${ }^{28}$, and a neutrino resulting in a daughter nucleus with one fewer proton while maintaining a constant mass number ${ }^{25}$. This positron will lose energy along its trajectory by collisions with matter until it slows enough to collide with an electron in an event called annihilation. Annihilation produces two $511 \mathrm{keV}$ collinear photons, which are emitted in directions $180^{\circ}$ from one another ${ }^{28}$. Because gamma rays are without mass and charge, they are barely attenuated along their path through, in this case, plant tissues and can be detected by coincidence detectors for dynamic PET imaging or by thallium-activated sodium iodide (NaI(Tl)) crystals for total activity quantification.

In terms of imaging positron decay, the spatial resolution is a direct result of the positron's kinetic energy. This is because the more energy a positron has, the farther distance it travels from the radiotracer location before annihilating, resulting in lower resolution ${ }^{28}$. To enhance the spatial resolution of positron imaging, autoradiography can be completed. Autoradiography was first done by accident in 1867 , before radioactivity was even discovered, when uranium salts 
blackened a film emulsion made of silver iodide and solver chloride ${ }^{29}$. The use of autoradiography as a technique to detect and image radiotracers really began just after World War II ${ }^{29}$. Modern phosphor films are no longer made with silver halide emulsions. The new phosphor, called Fuji's photostimulable phosphor, is made up of barium fluorobromide (BaFBr) with trace amounts of $\mathrm{Eu}^{2+}$ deposited on a flexible polyester film ${ }^{30}$. After exposure to the radioactive tissue, the $\mathrm{Eu}^{2+}$ ions are struck by ionizing radiation and lose an electron to become $\mathrm{Eu}^{3+}$ ions. These electrons enter the conduction band of the crystal and become trapped in a metastable state ${ }^{30}$. Now higher in energy than it was originally, a lower-frequency light insufficient in energy to ionize more $\mathrm{Eu}^{2+}$ ions (red light) can return the trapped electrons to the conduction band. When these electrons leave the conduction band, they release blue-violet light, which is detected by the phosphor imager, and a radio-image results. This technology enhances image resolution because exposing the plant tissue (or whatever biological tissue contains the radiotracer) to a film which will absorb the positron energy directly before annihilation occurs ${ }^{28}$, which minimizes the uncertainty in terms of distance the positron has traveled from the radiotracer localization site in the tissue. The trade-off in this imaging method is that rather than dynamic imaging, positron autoradiography results in a static image ${ }^{28}$, a snapshot in time.

In beta decay, the unstable nucleus will emit a beta particle, or electron originating in the nucleus, along with an anti-neutrino, which results in a daughter nucleus with one more proton but the same mass number as the parent nuclide ${ }^{25}$. The beta particle will carry energy through the surrounding plant tissue until it transfers all of its energy across multiple interactions with matter along the way. As a charged particle with mass, it will be more attenuated in plant tissues than gamma rays. Because there are no coincidence annihilation photons associated with beta decay 
PET imaging cannot be completed, only phosphor plate autoradiography, which has appreciable resolution but are only static images.

The $\mathrm{NaI}(\mathrm{Tl})$ detector, a scintillation detector, is also useful for total quantification of radionuclides that do not have annihilation photons (as well as those that do) presuming they have sufficient and detectable gamma rays associated with their radioactive decay schemes. In scintillation detection, a small percentage of the energy deposited in the scintillation crystals by the gamma rays is converted to light, which is subsequently converted into an electrical signal enhanced along photomultiplier tubes to elicit a signal ${ }^{25}$.

Disadvantages to using radiotracers must be considered and include radionuclide characteristics and availability as well as their potential health hazard to researchers utilizing them ${ }^{25}$. If the half-life of the desired radioisotope is very short, as in ${ }^{11} \mathrm{C}(20.4 \mathrm{~m})$ and ${ }^{18} \mathrm{~F}$ $(109.8 \mathrm{~m})$, it is necessary to perform the experiments at the same location as radionuclide production, or geographically close ${ }^{28}$. It is also true that the natural processes or changes investigated is limited by the lifespan of the radiotracer being such that radiotracers with halflives in terms of minutes can trace processes across a few hours but not much longer ${ }^{28}$. In addition, if the radioisotope is very long-lived, tool or instrument contamination can occur, which can lead to problems in their utility for other analyses. The available specific activity of a radionuclide is of great importance too, to avoid disturbing the system ${ }^{25}$. Finally, radiotracers pose radiation exposure risks, especially with shorter half-lives in which a great initial activity is required at the start of an experiment, thus shielding and safety precautions must be taken and can be costly ${ }^{25}$. Other challenges can include determining an appropriate radionuclide to use for the desired investigation. For example, tracing boron nutrient uptake and allocation is difficult as 
the radionuclides of boron that exist are not amenable for radiotracing due to their extremely short half-lives (e.g., ${ }^{8} \mathrm{~B}$ has the longest half-life at $770 \mathrm{~ms}$ ).

Some of the greatest advantages associated with radiotracer studies are their relative simplicity, extremely low limits of detection, and non-destructive, dynamic real-time information. A very small amount of radiotracer can be used to garner information, which allows investigators to avoid disturbing the living system. This is most beneficial when the radiotracer is chemically identical to the compound of interest. The ability to collect dynamic information allows for investigation of time-dependent processes and changes in the labeled plant's behavior and metabolism, which would otherwise be very challenging, if not impossible, to assess.

If investigations into plant metabolic processes which take place over longer time periods are desirable, or if a detection technique or instrument needs to avoid radiation contamination, stable isotope techniques can become desirable. The constraints are that samples must be destroyed in order to detect the elements or metabolites of interest and dynamic data is not achievable ${ }^{28}$. A combination of stable isotope detection via mass spectrometry techniques and radiotracer quantification and imaging can be powerful complementary tools which can bolster conclusions drawn from data and give information about immediate metabolism and physiology effects versus long term effects on plant productivity and nutrition.

\subsection{Plant Nutrient Needs and Plant Growth Promoting Bacteria as a Nutrition- Enhancement Approach}

Plant growth and development is dependent mostly on the combination and concentration of macro- and micronutrients (Table 1.3.1) in the soil ${ }^{31}$. Macronutrients such as nitrogen $(\mathrm{N})$, carbon $(\mathrm{C})$, phosphorus $(\mathrm{P})$, potassium $(\mathrm{K})$, etc. are the foundation of cellular components such as proteins, DNA, and lipids ${ }^{32}$. They are the elements required in largest quantity in plant 
nutrition, growth, and development. Micronutrients such as zinc ( $\mathrm{Zn})$, iron (Fe), boron (B), etc. are required in much smaller quantities in plants and typically function as cofactors for enzymes $^{31}$. All nutrients obtained by plant roots from the soil are known as mineral nutrients while those obtained from the air through photosynthesis, diffusion, and respiration are the nonmineral nutrients (oxygen $(\mathrm{O})$, carbon $(\mathrm{C})$, and hydrogen $(\mathrm{H}))^{32}$. Plants can take in and metabolize macro- and micronutrients differently due to a diverse range of factors influencing the efficiency of nutrient acquisition. Some plants have developed specialized adaptations for more successfully acquiring certain nutrients based on their natural environment soil conditions and nutrient limitations ${ }^{31}$.

\begin{tabular}{|c|c|c|c|}
\hline \multicolumn{2}{|c|}{$\begin{array}{l}\text { Macronutrient (>0.5 g kg plant dry } \\
\text { weight }^{-1} \text { ) }\end{array}$} & \multicolumn{2}{|c|}{$\begin{array}{l}\text { Micronutrient ( }<0.5 \mathrm{~g} \mathrm{~kg} \text { plant dry } \\
\text { weight }^{-1} \text { ) }\end{array}$} \\
\hline C & Carbon & $\mathrm{Fe}$ & Iron \\
\hline $\mathbf{H}$ & Hydrogen & $\mathrm{Mn}$ & Manganese \\
\hline $\mathbf{O}$ & Oxygen & $\mathrm{Cu}$ & Copper \\
\hline $\mathrm{N}$ & Nitrogen & $\mathrm{Zn}$ & Zinc \\
\hline $\mathrm{P}$ & Phosphorus & Mo & Molybdenum \\
\hline K & Potassium & B & Boron \\
\hline $\mathrm{S}$ & Sulfur & $\mathrm{Cl}$ & Chlorine \\
\hline $\mathrm{Ca}$ & Calcium & $\mathrm{Ni}$ & Nickel \\
\hline \multirow[t]{2}{*}{$\mathrm{Mg}$} & Magnesium & $\mathrm{Na}$ & Sodium \\
\hline & & Co & Cobalt \\
\hline
\end{tabular}

Table 1.3.1- Essential Nutrients for Plants. Macronutrients are located in the left-hand column with non-mineral nutrients appearing in bold text. Micronutrients appear in the right-hand column. 
As sessile organisms, plants encounter a variety of stresses such as drought, flooding, salinity and soil nutrient limitation, and must employ methods of adjustment in their location of growth. In fact, plants will experience such stresses over every developmental stage of their life span, and often even experience multiple stresses at once ${ }^{33}$. It is currently estimated that $30 \%$ of global crop production is lost as a result of abiotic stresses ${ }^{34}$. One approach to mitigation of such stresses, crop losses, and poor plant growth and performance can be found in plant growth promoting bacteria (PGPB). The literature establishes that many PGPB can strongly influence plant growth and increase crop yields. Various suggested mechanisms for such benefits to host plants include: enhancement of host micronutrient uptake from the soil ${ }^{35,36}$, induction of phytopathogen resistance ${ }^{37}$, greater host nitrogen uptake due to biological nitrogen fixation $(\mathrm{BNF})^{38}$, and phytostimulation through the microbial production of plant growth hormones such as auxin, cytokinins, giberellins, and nitric oxide $^{17,39,40}$.

There are many different PGPB common in plant rhizospheres, which associate epiphytically or endophytically in high numbers $\left(\sim 10^{8} \mathrm{gfw}^{-1}\right)$ without the induction of plant defense responses ${ }^{41-43}$. One Gram-negative bacteria, Azospirillum brasilense, is one of the most wellknown PGPB, a diazotrophic epiphyte. This bacterium is capable of BNF and can also produce the plant growth hormone, auxin ${ }^{38}$.

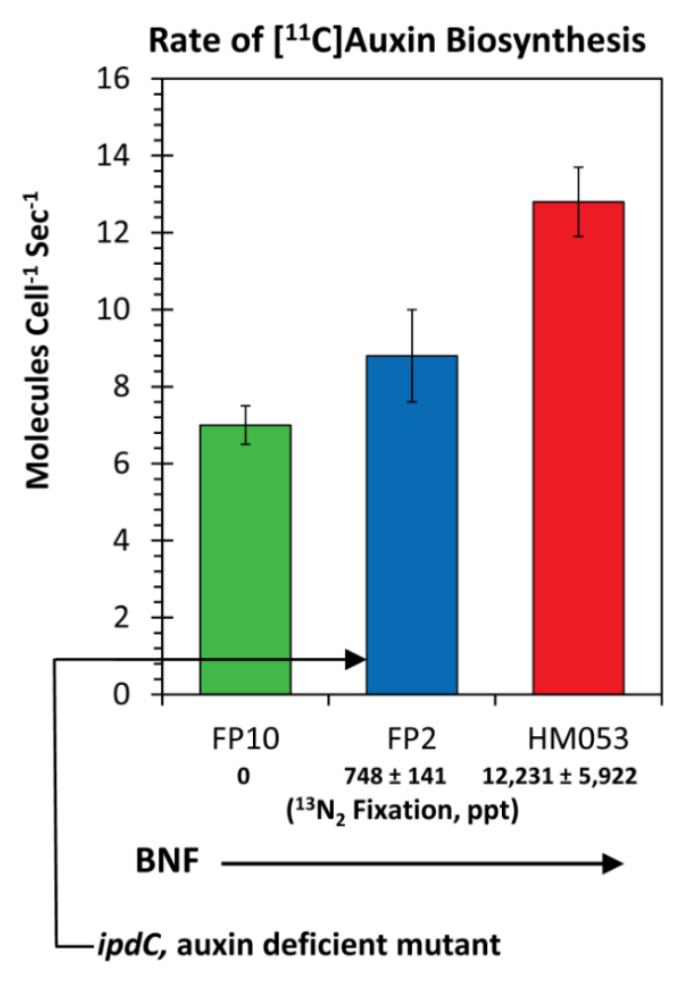

Figure 1.3.1- Comparison of auxin production capacities of Azospirillum brailense functional mutants $^{40}$ 
Commercial inoculants of various functional strains of this bacteria have been characterized and tested over the years for their impact on grain crops, like maize, under a series of environmental conditions $^{44-46}$. This work relies on $A$. brasilense functional mutants across a spectrum of biological functions spanning varying levels of auxin biosynthetic capacity to BNF capacity (Figure 1.3.1 $)^{40}$. The use of functional mutants to examine plant-microbial interactions has been used extensively in other PGPB systems to examine the effects of associated microorganisms on plant physiology ${ }^{47}$. The functional mutants of $A$. brasilense used this work include HM053, a Nif ${ }^{+}$constitutively expressed strain hyper-fixing $\mathrm{N}_{2}$ and producing high levels of auxin; FP10, a Nif ${ }^{-}$ strain deficient in $\mathrm{N}_{2}$-fixation, but produces auxin albeit at a lower capacity than HM053; and $i p d C$, a strain significantly reduced in its capacity to produce auxin along the indole-3-pyruvic acid pathway while maintaining wild-type levels of BNF capacity.

\subsection{Perspective and Purpose}

In thirty years, the current global food production will be insufficient to fully feed the projected population of 9.1 billion people ${ }^{48}$. Maintaining our food security requires the global agricultural industry to increase production by at least $60 \%$ while combating water scarcity, climate change, and soil micronutrient deficiencies. Cereal grains like rice and corn are particularly important as they provide nearly a third of all food calories to more than 4.5 billion people living in developing countries ${ }^{49}$. Excessive use of fertilizers and exhaustive cultivation of high-yield crops has led to severe soil micronutrient deficiencies in approximately $40 \%$ of all arable land, especially of iron and zinc ${ }^{50}$. Meanwhile, salinity is an ever increasing threat to food security as climate change conditions drive an excess accumulation of salt ${ }^{51}$.

This dissertation research explores maize nutrient uptake and allocation with the tools of radiotracer and radio-imaging technologies combined with stable isotope and other analytical 
techniques. It also explores the utilization of plant growth promoting bacteria (PGPB) to mitigate abiotic stresses such as various soil nutrient limitations and excess soil salinity with the advantages of faster deployment to the field over typical crop-breeding methods while being a socially preferred alternative to genetic modification. While evidence exists that certain beneficial microbes can influence plant growth through improved micronutrient uptake, we know little about the mechanisms of action, which, if fundamentally understood, could lay the foundation for translating this approach to general farming practices. Radiotracers are a unique tool for the investigation of plant nutrition, metabolism, and plant-microbe interactions due to their precise, dynamic, and non-disruptive nature. They allow insight into the kinetics of micronutrient uptake and plant metabolism, micronutrient localization in whole plant tissues, as well as a measurements of carbon metabolic flux, which are unique to our lab and very few others worldwide.

\subsection{Abbreviations}

$\begin{array}{ll}{ }^{18} \text { F]FDG } & \text { F-18 labeled fluorodeoxyglucose } \\ \text { PET } & \text { Positron Emission Tomography } \\ \text { NaI(TI) } & \text { Thallium-activated sodium iodide } \\ \text { PGPB } & \text { Plant growth promoting bacteria } \\ \text { BNF } & \text { Biological nitrogen fixation }\end{array}$




\section{References}

(1) Foundation, C.-12. Importance of Plants |CK-12 Foundation https://flexbooks.ck12.org/cbook/ck-12-biology-flexbook2.0/section/9.2/primary/lesson/importance-of-plants-bio (accessed Feb 19, 2021).

(2) Gest, H. Samuel Ruben's Contributions to Research on Photosynthesis and Bacterial Metabolism with Radioactive Carbon. Photosynthesis Research 2004, 80 (1), 77.

(3) Calvin, M.; Benson, A. A. The Path of Carbon in Photosynthesis. Science 1948, 107 (2784), 476-480.

(4) Minchin, P. E. H.; Thorpe, M. R. Using the Short-Lived Isotope ${ }^{11}$ C in Mechanistic Studies of Photosynthate Transport. Funct Plant Biol 2003, 30 (8), 831-841.

(5) Babst, B. A.; Ferrieri, R. A.; Gray, D. W.; Lerdau, M.; Schlyer, D. J.; Schueller, M.; Thorpe, M. R.; Orians, C. M. Jasmonic Acid Induces Rapid Changes in Carbon Transport and Partitioning in Populus. New Phytologist 2005, 167 (1), 63-72.

(6) Ba, B.; Ra, F.; Cm, O.; Mr, T. Lymantria Dispar Herbivory Induces Rapid Changes in Carbon Transport and Partitioning in Populus Nigra. Entomol Exp Appl 2008, 128 (1), 117-125.

(7) Ferrieri, R. A.; Gray, D. W.; Babst, B. A.; Schueller, M. J.; Schlyer, D. J.; Thorpe, M. R.; Orians, C. M.; Lerdau, M. Use of Carbon-11 in Populus Shows That Exogenous Jasmonic Acid Increases Biosynthesis of Isoprene from Recently Fixed Carbon. Plant, Cell \& Environment 2005, 28 (5), 591-602.

(8) Gómez, S.; Ferrieri, R. A.; Schueller, M.; Orians, C. M. Methyl Jasmonate Elicits Rapid Changes in Carbon and Nitrogen Dynamics in Tomato. New Phytol 2010, 188 (3), 835-844.

(9) Gómez, S.; Steinbrenner, A. D.; Osorio, S.; Schueller, M.; Ferrieri, R. A.; Fernie, A. R.; Orians, C. M. From Shoots to Roots: Transport and Metabolic Changes in Tomato after Simulated Feeding by a Specialist Lepidopteran. Entomologia Experimentalis et Applicata 2012, 144 (1), 101-111.

(10) Hanik, N.; Best, M.; Schueller, M. J.; Tappero, R.; Ferrieri, R. A. Defense Priming in Nicotiana Tabacum Accelerates and Amplifies 'New' C/N Fluxes in Key Amino Acid Biosynthetic Pathways. Plants 2020, 9 (7), 851.

(11) Robert, C. a. M.; Ferrieri, R. A.; Schirmer, S.; Babst, B. A.; Schueller, M. J.; Machado, R. A. R.; Arce, C. C. M.; Hibbard, B. E.; Gershenzon, J.; Turlings, T. C. J.; Erb, M. Induced Carbon Reallocation and Compensatory Growth as Root Herbivore Tolerance Mechanisms. Plant, Cell \& Environment 2014, 37 (11), 2613-2622.

(12) Qu, W.; Robert, C. A. M.; Erb, M.; Hibbard, B. E.; Paven, M.; Gleede, T.; Riehl, B.; Kersting, L.; Cankaya, A. S.; Kunert, A. T.; Xu, Y.; Schueller, M. J.; Shea, C.; Alexoff, D.; Lee, S. J.; Fowler, J. S.; Ferrieri, R. A. Dynamic Precision Phenotyping Reveals Mechanism of Crop Tolerance to Root Herbivory. Plant Physiology 2016, 172 (2), 776-788. 
(13) Song, L.; Agtuca, B.; Schueller, M. J.; Jurisson, S. S.; Stacey, G.; Ferrieri, R. A.

Relationship Between Carbon Mobilization and Root Growth Measured by Carbon-11 Tracer in Arabidopsis Starch Mutants. J Plant Growth Regul 2019, 38 (1), 164-179.

(14) Thorpe, M. R.; Ferrieri, A. P.; Herth, M. M.; Ferrieri, R. A. ${ }^{11}$ C-Imaging: Methyl Jasmonate Moves in Both Phloem and Xylem, Promotes Transport of Jasmonate, and of Photoassimilate Even after Proton Transport Is Decoupled. Planta 2007, 226 (2), 541-551.

(15) Agtuca, B.; Rieger, E.; Hilger, K.; Song, L.; Robert, C. A. M.; Erb, M.; Karve, A.; Ferrieri, R. A. Carbon-11 Reveals Opposing Roles of Auxin and Salicylic Acid in Regulating Leaf Physiology, Leaf Metabolism, and Resource Allocation Patterns That Impact Root Growth in Zea Mays. J Plant Growth Regul 2014, 33 (2), 328-339.

(16) Housh, A. B.; Powell, G.; Scott, S.; Anstaett, A.; Gerheart, A.; Benoit, M.; Waller, S.; Powell, A.; Guthrie, J. M.; Higgins, B.; Wilder, S. L.; Schueller, M. J.; Ferrieri, R. A. Functional Mutants of Azospirillum Brasilense Elicit Beneficial Physiological and Metabolic Responses in Zea Mays Contributing to Increased Host Iron Assimilation. The ISME Journal 2021, 1-18.

(17) Pankievicz, V. C. S.; do Amaral, F. P.; Santos, K. F. D. N.; Agtuca, B.; Xu, Y.; Schueller, M. J.; Arisi, A. C. M.; Steffens, M. B. R.; de Souza, E. M.; Pedrosa, F. O.; Stacey, G.; Ferrieri, R. A. Robust Biological Nitrogen Fixation in a Model Grass-Bacterial Association. Plant J 2015, 81 (6), 907-919.

(18) Lelie, D. van der; Taghavi, S.; Monchy, S.; Schwender, J.; Miller, L.; Ferrieri, R.; Rogers, A.; Wu, X.; Zhu, W.; Weyens, N.; Vangronsveld, J.; Newman, L. Poplar and Its Bacterial Endophytes: Coexistence and Harmony. Critical Reviews in Plant Sciences 2009, 28 (5), 346358. https://doi.org/10.1080/07352680903241204.

(19) Waller, S.; Wilder, S. L.; Schueller, M. J.; Housh, A. B.; Ferrieri, R. A. Quantifying PlantBorne Carbon Assimilation by Root-Associating Bacteria. Microorganisms 2020, 8 (5), 700.

(20) Hevesy, G. The Absorption and Translocation of Lead by Plants. Biochem J 1923, 17 (4-5), 439-445.

(21) Ariño-Estrada, G.; Mitchell, G. S.; Saha, P.; Arzani, A.; Cherry, S. R.; Blumwald, E.; Kyme, A. Z. Imaging Salt Uptake Dynamics in Plants Using PET. Scientific Reports 2019, 9 (1), 18626.

(22) Parveen, S.; Yadav, P.; Singh, B. Radiochemical Evidence for the Contribution of Iron (Using 59Fe) Remobilization Efficiency towards Nitrogen (N) and Fe Deficiency Tolerance in Wheat. Radiochimica Acta 2019, 107 (5), 431-439.

(23) Fowler, J. S.; Ido, T. Initial and Subsequent Approach for the Synthesis of ${ }^{18}$ FDG. Seminars in Nuclear Medicine 2002, 32 (1), 6-12.

(24) Fatangare, A.; Svatoš, A. Applications of 2-Deoxy-2-Fluoro-D-Glucose (FDG) in Plant Imaging: Past, Present, and Future. Front. Plant Sci. 2016, 7. 
(25) Loveland, W.; Morrisey, D. J.; Seaborg, G. T. Modern Nuclear Chemistry; John Wiley \& Sons, INC.: Hoboken, NJ, 2006.

(26) O’Leary, M. H. Carbon Isotopes in Photosynthesis: Fractionation Techniques May Reveal New Aspects of Carbon Dynamics in Plants. BioScience 1988, 38 (5), 328-336.

(27) Caldelas, C.; Weiss, D. J. Zinc Homeostasis and Isotopic Fractionation in Plants: A Review. Plant Soil 2017, 411 (1-2), 17-46.

(28) Kiser, M. R.; Reid, C. D.; Crowell, A. S.; Phillips, R. P.; Howell, C. R. Exploring the Transport of Plant Metabolites Using Positron Emitting Radiotracers. HFSP J 2008, 2 (4), 189204.

(29) AUTORADIOGRAPHY

https://lab.anhb.uwa.edu.au/hb313/main_pages/timetable/lectures/autoradiography.htm (accessed Mar 5, 2021).

(30) FUJIFILM WORLDWIDE - Products - Science Imaging Systems - What is Imaging Plate? https://web.archive.org/web/20060319120039/http://home.fujifilm.com/products/science/ip/princ iple.html (accessed Mar 5, 2021).

(31) Plant-Soil Interactions: Nutrient Uptake | Learn Science at Scitable https:/www.nature.com/scitable/knowledge/library/plant-soil-interactions-nutrient-uptake105289112/ (accessed Feb 19, 2021).

(32) 31.1C: Essential Nutrients for Plants

https://bio.libretexts.org/Bookshelves/Introductory_and_General_Biology/Book\%3A_General_B iology_(Boundless)/31\%3A_Soil_and_Plant_Nutrition/31.1\%3A_Nutritional_Requirements_of_ Plants/31.1C\%3A_Essential_Nutrients_for_Plants (accessed Mar 4, 2021).

(33) Sharma, P.; Jha, A. B.; Dubey, R. S.; Pessarakli, M. Reactive Oxygen Species, Oxidative Damage, and Antioxidative Defense Mechanism in Plants under Stressful Conditions https://www.hindawi.com/journals/jb/2012/217037/ (accessed Feb 18, 2021).

(34) Goswami, D.; Thakker, J. N.; Dhandhukia, P. C. Portraying Mechanics of Plant Growth Promoting Rhizobacteria (PGPR): A Review. Cogent Food \& Agriculture 2016, 2 (1), 1127500.

(35) Brusamarello-Santos, L. C. C.; Alberton, D.; Valdameri, G.; Camilios-Neto, D.; Covre, R.; Lopes, K. de P.; Zibetti Tadra-Sfeir, M.; Faoro, H.; Adele Monteiro, R.; Barbosa-Silva, A.; John Broughton, W.; Oliveira Pedrosa, F.; Wassem, R.; Souza, E. M. de. Modulation of Defence and Iron Homeostasis Genes in Rice Roots by the Diazotrophic Endophyte Herbaspirillum Seropedicae. Sci Rep 2019, 9 (1), 10573.

(36) Singh, D.; Prasanna, R. Potential of Microbes in the Biofortification of Zn and Fe in Dietary Food Grains. A Review. Agron. Sustain. Dev. 2020, 40 (2), 15.

(37) Verhagen, B. W. M.; Glazebrook, J.; Zhu, T.; Chang, H.-S.; van Loon, L. C.; Pieterse, C. M. J. The Transcriptome of Rhizobacteria-Induced Systemic Resistance in Arabidopsis. Mol Plant Microbe Interact 2004, 17 (8), 895-908. 
(38) Fibach-Paldi, S.; Burdman, S.; Okon, Y. Key Physiological Properties Contributing to Rhizosphere Adaptation and Plant Growth Promotion Abilities of Azospirillum Brasilense. FEMS Microbiology Letters 2012, 326 (2), 99-108.

(39) Richardson, A.; Barea, J.; Mcneill, A.; Prigent-Combaret, C. Richardson AE, Barea JM, McNeill AM, Prigent-Combaret C.. Acquisition of Phosphorus and Nitrogen in the Rhizosphere and Plant Growth Promotion by Microorganisms. Plant Soil 321: 305-339. Plant and Soil 2009, 321.

(40) Housh, A. B.; Powell, G.; Scott, S.; Anstaett, A.; Gerheart, A.; Benoit, M.; Waller, S.; Powell, A.; Guthrie, J. M.; Higgins, B.; Wilder, S. L.; Schueller, M. J.; Ferrieri, R. A. Functional Mutants of Azospirillum Brasilense Elicit Beneficial Physiological and Metabolic Responses in Zea Mays Contributing to Increased Host Iron Assimilation. ISME J 2021, 1-18.

(41) do Amaral, F. P.; Agtuca, B. J.; Stacey, G. Setaria Root-Microbe Interactions. In Genetics and Genomics of Setaria; Doust, A., Diao, X., Eds.; Plant Genetics and Genomics: Crops and Models; Springer International Publishing: Cham, 2017; pp 239-250.

(42) Reinhold-Hurek, B.; Hurek, T. Life in Grasses: Diazotrophic Endophytes. Trends Microbiol 1998, 6 (4), 139-144.

(43) Reinhold-Hurek, B.; Hurek, T. Living inside Plants: Bacterial Endophytes. Curr Opin Plant Biol 2011, 14 (4), 435-443.

(44) Bashan, Y.; Holguin, G.; de-Bashan, L. E. Azospirillum-Plant Relationships: Physiological, Molecular, Agricultural, and Environmental Advances (1997-2003). Can J Microbiol 2004, 50 (8), 521-577.

(45) Hungria, M.; Campo, R. J.; Souza, E. M.; Pedrosa, F. O. Inoculation with Selected Strains of Azospirillum Brasilense and A. Lipoferum Improves Yields of Maize and Wheat in Brazil. Plant Soil 2010, 331 (1-2), 413-425.

(46) Dobbelaere, S.; Croonenborghs, A.; Thys, A.; Ptacek, D.; Vanderleyden, J.; Dutto, P.; Labandera-Gonzalez, C.; Caballero-Mellado, J.; Aguirre, J. F.; Kapulnik, Y.; Brener, S.; Burdman, S.; Kadouri, D.; Sarig, S.; Okon, Y. Responses of Agronomically Important Crops to Inoculation with Azospirillum. Functional Plant Biol. 2001, 28 (9), 871-879.

(47) Ravanbakhsh, M.; Kowalchuk, G. A.; Jousset, A. Root-Associated Microorganisms Reprogram Plant Life History along the Growth-Stress Resistance Tradeoff. ISME J 2019, 13 (12), 3093-3101.

(48) Food security and why it matters https://www.weforum.org/agenda/2016/01/food-securityand-why-it-matters/ (accessed Feb 19, 2021).

(49) Shiferaw, B.; Prasanna, B. M.; Hellin, J.; Bänziger, M. Crops That Feed the World 6. Past Successes and Future Challenges to the Role Played by Maize in Global Food Security. Food Sec. 2011, 3 (3), 307. 
(50) Cakmak, I. Plant Nutrition Research: Priorities to Meet Human Needs for Food in Sustainable Ways. Plant and Soil 2002, 247 (1), 3-24.

(51) Zhu, J.-K. Salt and Drought Stress Signal Transduction in Plants. Annu Rev Plant Biol 2002, $53,247-273$. 


\section{Chapter Two: Assessment of a $\left[{ }^{18} \mathrm{~F}\right]$-4-Fluorophenylboronic Acid Radiotracer for Imaging Boron in Maize}

\subsection{Preface}

This project was published on February 1 $1^{\text {st }}, 2020$ in the International Journal of Molecular Sciences (IJMS). As first author on the manuscript, I was involved in the radiotracer development and assessment, administration of the radiotracer to live plants and subsequent autoradiographic imaging, measurement of B in the plant tissues via ICP-MS, writing of the first draft of the manuscript, and subsequent edits. All Arabidopsis B deficiency symptoms and growth response assays are vital to the project conclusions and were completed and contributed by Dr. Michaela S. Matthes of the Paula McSteen, PhD research group within the University of Missouri Division of Plant Sciences.

Housh, A. B.; Matthes, M. S.; Gerheart, A.; Wilder, S. L.; Kil, K.; Schueller, M.; Guthrie, J. M.; McSteen, P.; Ferrieri, R. A. Assessment of a ${ }^{18}$ F-Phenylboronic Acid Radiotracer for Imaging Boron in Maize. International Journal of Molecular Sciences 21, 976 (2020).

\subsection{Introduction}

The trace mineral boron (B) is an essential micronutrient required for plant fitness and crop yield ${ }^{1-2}$, as well as animal and human health ${ }^{3}$. B is a weak Lewis acid and is accessible to the plant in the form of boric acid (BA) and as the borate anion $\left(\mathrm{BO}_{3}{ }^{3-}\right)$. Deficiency of $\mathrm{B}$ in the soil is a factor affecting crop yield worldwide and is reported to be the most widespread micronutrient deficiency ${ }^{4}$. Yet knowledge about the underlying causes and the cellular functions of B is still limited. The best characterized cellular function of B is the crosslinking of pectic subunits, called Rhamnogalacturonan-II (RG-II), in the cell wall ${ }^{5-7}$. Cell wall crosslinking by B is needed for proper cell wall stability and plant development ${ }^{8}$. B binding sites in the cell wall are 
limited and the observation that B levels in the cell wall do not change with altered B levels in the media, while cytosolic levels do, suggests additional roles of B beyond that of the cell wall crosslinking ${ }^{9}$. Indeed, additional roles of B in sugar transport, transpiration, lignification, the metabolism of carbohydrates, RNA, indole-3-acetic acid (IAA), phenols, and as a membrane component have been suggested and demonstrated ${ }^{10-11}$.

Plants respond to B deficiency with a cessation of growth at the growing tips, or meristems, in both the root and the shoot ${ }^{12}$. Meristems are groups of stem cells that eventually give rise to all postembryonic above and below the ground organs ${ }^{13}$. In Arabidopsis, one of the first phenotypic defects of B deficiency is the inhibition of root elongation through effects on the root apical meristem $^{14}$. In maize, B deficiency also affects root growth and reduces crop yields ${ }^{15-16}$. Conversely, treatment of sunflower with BA stimulates adventitious root growth ${ }^{17}$. While there are benefits to increased $\mathrm{B}$, too much is toxic and levels must be carefully controlled by the plant $^{18-19}$.

$\mathrm{B}$ is absorbed by roots as undissociated BA either passively via diffusion when B concentrations are adequate or high, or actively under B deficiency conditions by B import facilitators and export proteins ${ }^{19}$. AtBOR1, the first characterized B transporter in Arabidopsis ${ }^{20}$, exports borate out of the cell. AtNIP5;1 belongs to the major intrinsic protein family and facilitates the import of $\mathrm{BA}^{21}$. B itself regulates uptake by controlling mRNA degradation and protein stability of the transporters ${ }^{19}$. The $\mathrm{B}$ responsivity of $A t \mathrm{NIP} 5 ; 1$ was recently used to develop a B sensor that responds to cytosolic B levels ${ }^{22}$. BOR1 and NIP5 homologs have been identified in several species including maize. The AtBOR1 co-ortholog in maize is called Zmrottenear (Zmrte) and the AtNIP5;1 co-ortholog in maize is called Zmtassel-less1 (Zmtls 1) ${ }^{23-}$ 25. 
One approach to decipher the role of B in plants is to induce B deficiency experimentally. Successful strategies to induce B deficiency have been to use inherently B deficient mutants, like the Atnip 5; 1, Atbor1, Zmtls1, or Zmrte mutants ${ }^{20,21,23-25}$. In recent years, chemical approaches to inducing B deficiency, such as the use of phenylboronic acid (PBA) have been published in petunia $^{26}$, apple ${ }^{27}$, and Arabidopsis ${ }^{28,29}$. PBA lacks the third hydroxyl functional group in its chemical structure and therefore fails to generate bridging cis-diol complexes in the cell walls resulting in a $\mathrm{B}$ deficiency response ${ }^{26}$. The use of this chemical to induce $\mathrm{B}$ deficiency in maize has not been explored.

One reason the cellular functions of B have not been fully resolved is because B is present at low levels in plants ${ }^{30}$. Methods to visualize or quantify B, therefore, have limitations in sensitivity, standardization, reproducibility, and tissue destruction ${ }^{2}$. Past successes in quantifying B in soil and plant systems have mainly been through photometric methods quantified via UV$\mathrm{VIS}^{2,30}$. Other quantification methods such as inductively coupled plasma-mass spectrometry (ICP-MS) or optical emission spectrometry (ICP-OES) have also been used for B quantification in soil and plant tissues, but are destructive and indicate only total B in digested tissue rather than spatial localization of B. Imaging and quantification by chemical methods such as fluorescence and low-energy $\mathrm{x}$-ray fluorescence have not been successful as the natural concentration of B in plants is beneath their detection limits ${ }^{31}$. The same holds true for advanced microscopy techniques like scanning electron microscopy-electron energy loss spectroscopy or transmission electron microscopy (Matthes, McSteen, White, and Schaufflinger, unpublished results). Imaging $\mathrm{B}$ in plant systems has been accomplished by thermal neutron radiography in maize $\mathrm{e}^{32}$ and clover $^{33}$, but high resolution was not achieved. The use of a radiotracer could overcome the limitations of tissue destruction and resolution; however, there are significant challenges for the 
synthesis of a B radiotracer that works in plant systems. First, radionuclides of B are not suitable for incorporation into radiotracers as the longest half-life $\left(\mathrm{t}_{1 / 2}\right)$ belongs to ${ }^{8} \mathrm{~B}$ at $770 \mathrm{~ms}^{34}$, which limits synthesis, purification, and administration to plant systems. Second, since B is found as BA, a logical alternative would be to label oxygen $(\mathrm{O})$ rather than $\mathrm{B}$, but the longest lived radioisotope of $\mathrm{O},{ }^{15} \mathrm{O}$, has a $\mathrm{t}_{1} / 2=2 \mathrm{~m}^{34}$. Labeling hydrogen is not an alternative as it will be lost when B incorporates into the cell wall or reacts with other species in planta in the form of the borate anion.

Here we test the ability to utilize fluorine-18 labelled 4-fluorophenylboronic acid $\left(\left[{ }^{18} \mathrm{~F}\right] \mathrm{FPBA}\right)$ as a radioactive tracer for $\mathrm{B}$ visualization and the consequences of the induction of B deficiency by PBA and unlabeled 4-fluorophenylboronic acid (FPBA) on maize root development. FPBA is a structural analog of PBA where a fluorine group is added across the aromatic ring from the BA group and is more amenable for radiolabeling as fluorine-18 $\left[{ }^{18} \mathrm{~F}\right]$ chemistry is well established and relatively accessible.

\subsection{Results}

Synthesis and Assessment of a $\left[{ }^{18} \mathrm{~F}\right]$-Phenylboronic Acid Radiotracer- We hypothesized that PBA could be radioactively labelled in order to use it as a B radiotracer. As previously published, the radioactive $\left[{ }^{18} \mathrm{~F}\right]$ fluoride can be incorporated onto the boranate ester of the PBA via a copper-mediated radiofluorination ${ }^{35}$. The $\mathrm{t}_{1 / 2}$ of $\left[{ }^{18} \mathrm{~F}\right]$ is sufficiently long at 110 min enabling complex radiochemistry and use of the final radiotracer for imaging purposes ${ }^{34}$. In our experiments $\left[{ }^{18} \mathrm{~F}\right] \mathrm{FPBA}$ was synthesized in approximately $10 \mathrm{~min}$ from 1,4-phenyldiboronic acid precursor, with purification and quantification complete after an average of $90 \mathrm{~min}$ (Figure

2.3.1. A). Specific activity, or measurement comparing radioactive labeled substrate to unlabeled substrate, of this radiotracer was $1.04 \times 10^{6} \mathrm{Ci} \mathrm{mol}^{-1}\left(3.85 \times 10^{16} \mathrm{~Bq} \mathrm{~mol}^{-1}\right)$ as calculated to end of bombardment. This is a valid specific activity for tracing and imaging of $\mathrm{B}$, as it is well 
beneath the expected concentration of B in plant tissues (10-100 $\mathrm{mg} \mathrm{kg}^{-1} \mathrm{dry}$ weight $\left.{ }^{2}\right)$. In contrast to published syntheses of $\left[{ }^{18} \mathrm{~F}\right] \mathrm{FPBA}^{35}$, we added an additional purification step to the tracer production, although with limited success. A Chelex ${ }^{\circledR}$ column removed much of the copper catalyst from the solution prior to separation on the high performance liquid chromatography (HPLC) semi-prep column.

$$
\text { A }
$$<smiles>O=[Se]c1ccc([18OH])cc1</smiles>

$\mathrm{K}^{18} \mathrm{~F} / \mathrm{K}(\mathrm{OTf}), \mathrm{K}_{2} \mathrm{CO}_{3}$ $\mathrm{Cu}(\mathrm{OTf})_{2}$, pyridine

DMF

$110^{\circ} 10 \mathrm{~min}$<smiles>O=C(O)c1ccc(Br)cc1</smiles>

B

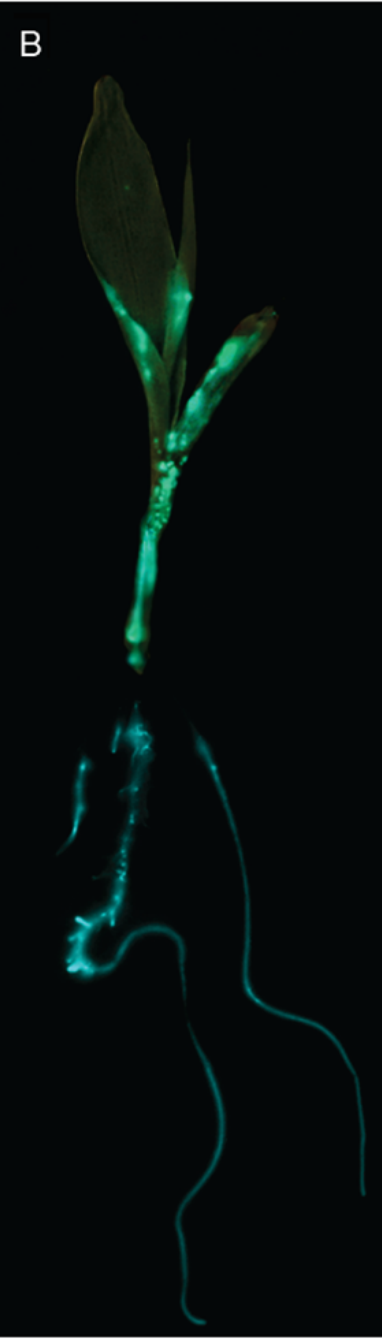

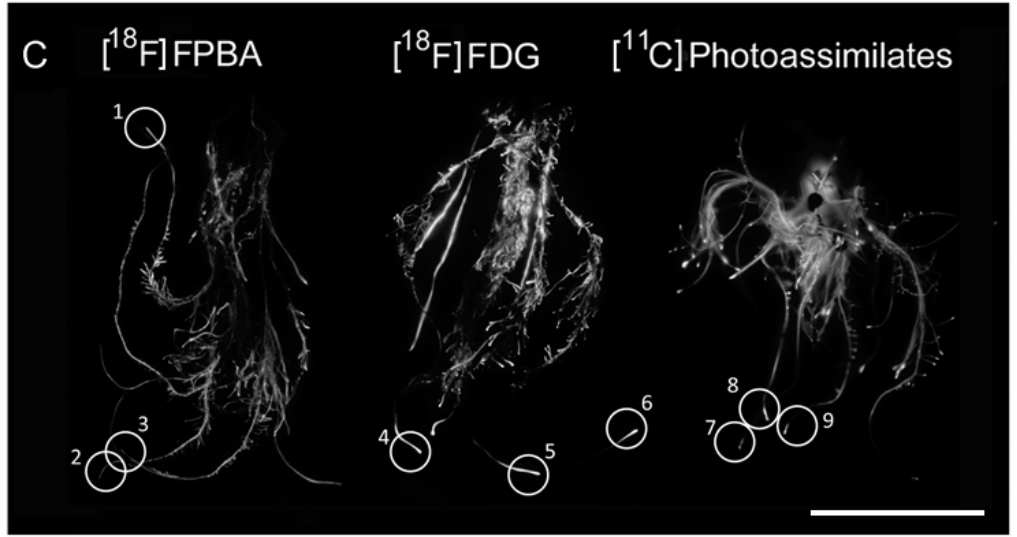

D

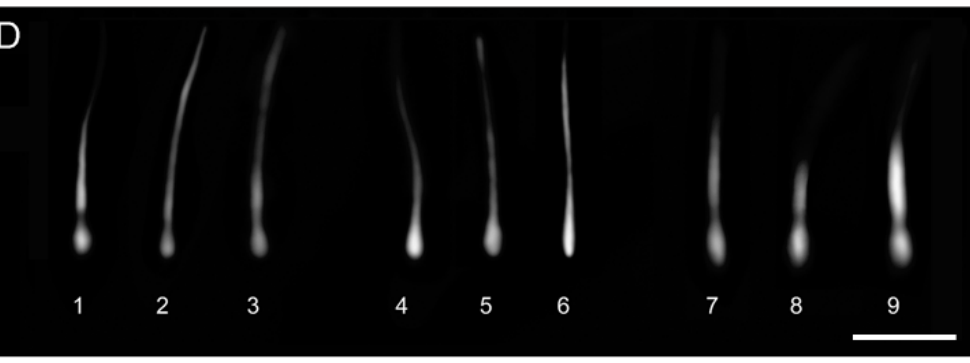

Figure 2.3.1.: Synthesis and Administration of a $\left[{ }^{18} \mathrm{~F}\right] \mathrm{FPBA}$ radiotracer to maize seedlings (inbred B73) A) Single vial synthesis of FPBA from precursor 1,4-diboronic acid using a copper catalyst. B) Radiographic image overlaid on visual image of five days old maize seedling after passive hydroponic administration of $\left[{ }^{18} \mathrm{~F}\right] \mathrm{FPBA}$ radiotracer. C) Threeweek old maize root radiographic images under passive administration of $\left[{ }^{18} \mathrm{~F}\right] \mathrm{FPBA}$, $\left[{ }^{18} \mathrm{~F}\right] \mathrm{FDG}$, and $\left[{ }^{11} \mathrm{C}\right] \mathrm{CO}_{2}$. Regions of interest appearing in circles are shown under greater magnification in panel D. D) Primary root tips and elongation zones as they appear under radiographic imaging using $\left[{ }^{18} \mathrm{~F}\right]-\mathrm{PBA},\left[{ }^{18} \mathrm{~F}\right] \mathrm{FDG}$, and $\left[{ }^{11} \mathrm{C}\right] \mathrm{CO}_{2}$. Scale bar in $\mathrm{C}=15 \mathrm{~cm}$ and in $\mathrm{D}=0.5 \mathrm{~cm}$. 
Variations on the synthesis were tested for maximal percent yield while balancing the effects of the half-life of F-18. First, the reaction solvent was chosen in radioactive iterations of varied reaction conditions (Table 2.3.1.). Time courses were set up in which $\left[{ }^{18} \mathrm{~F}\right] \mathrm{FPBA}$ was made using both DMF and DMSO at $110^{\circ} \mathrm{C}$ across 5,10 , and 20 minutes reaction times. The yield was consistently greater when conducted in DMF, thus it became the reaction solvent.

\begin{tabular}{|c|c|c|c|}
\hline $\begin{array}{l}\text { Solvent of reaction } \\
\text { solution }\end{array}$ & Temperature $\left({ }^{\circ} \mathbf{C}\right)$ & Time of Reaction (min) & Yield as measured by TLC (\%) \\
\hline DMF & 110 & 5 & 15 \\
\hline DMSO & 110 & 5 & 4 \\
\hline DMF & 100 & 10 & 16 \\
\hline DMSO & 110 & 10 & 3 \\
\hline DMF & 110 & 20 & 11 \\
\hline DMSO & 110 & 20 & 7 \\
\hline
\end{tabular}

Table 2.3.1.- Reactions conditions investigating solvents for maximum yield

Once the reaction solvent was selected, reaction temperature and time were evaluated for maximum for time reacted (Table 2.3.2.). Longer reaction times were explored up to $30 \mathrm{~min}$ but no increase in yield was observed after $10 \mathrm{~min}$. Because of the nature of radioactivity, a synthesis should be as short as possible for maximization of product formed and specific activity of the final product. While $\left[{ }^{18} \mathrm{~F}\right]$-fluoride was produced on site via a cyclotron for the radiochemistry, it is possible to order the radionuclide and have it delivered if no access to a cyclotron exists, providing the proper licensing and safety protocols are followed. In the United States there now exist several regional distribution centers that can provide this resource. Thus the tracer could be available to researchers who do not have access to a cyclotron. 


\begin{tabular}{|c|c|c|}
\hline Temperature $\left({ }^{\circ} \mathbf{C}\right)$ & Time of Reaction (min) & Yield as measured by TLC (\%) \\
\hline 100 & 5 & 12 \\
\hline 110 & 5 & 15 \\
\hline 100 & 10 & 13 \\
\hline 110 & 10 & 16 \\
\hline 100 & 20 & 19 \\
\hline 110 & 20 & 11 \\
\hline 100 & 30 & 5 \\
\hline 110 & 30 & 9 \\
\hline
\end{tabular}

Table 2.3.2.- Reaction conditions investigating reaction time and temperature for maximum yield

Localization of $\left[{ }^{18} \mathrm{~F}\right]-P B A$ in Maize Lateral Root Initiation Sites and Leaf Edges- Approximately $200 \mu \mathrm{Ci}$ of the synthesized $\left[{ }^{18} \mathrm{~F}\right] \mathrm{FPBA}$ tracer was hydroponically administered to the root system of maize plants (inbred B73) either at five days post-germination (Figures 2.3.1. B and 2.3.2.) or three weeks post-germination (Figures 2.3.1. C-D and 2.3.3.) by suspending the plant roots in the tracer-water. As a comparison, 2-deoxy-2-[ $\left[{ }^{18} \mathrm{~F}\right]$ fluoro-D-glucose $\left(\left[{ }^{18} \mathrm{~F}\right] \mathrm{FDG}\right)$ and $\left[{ }^{11} \mathrm{C}\right]$ carbon

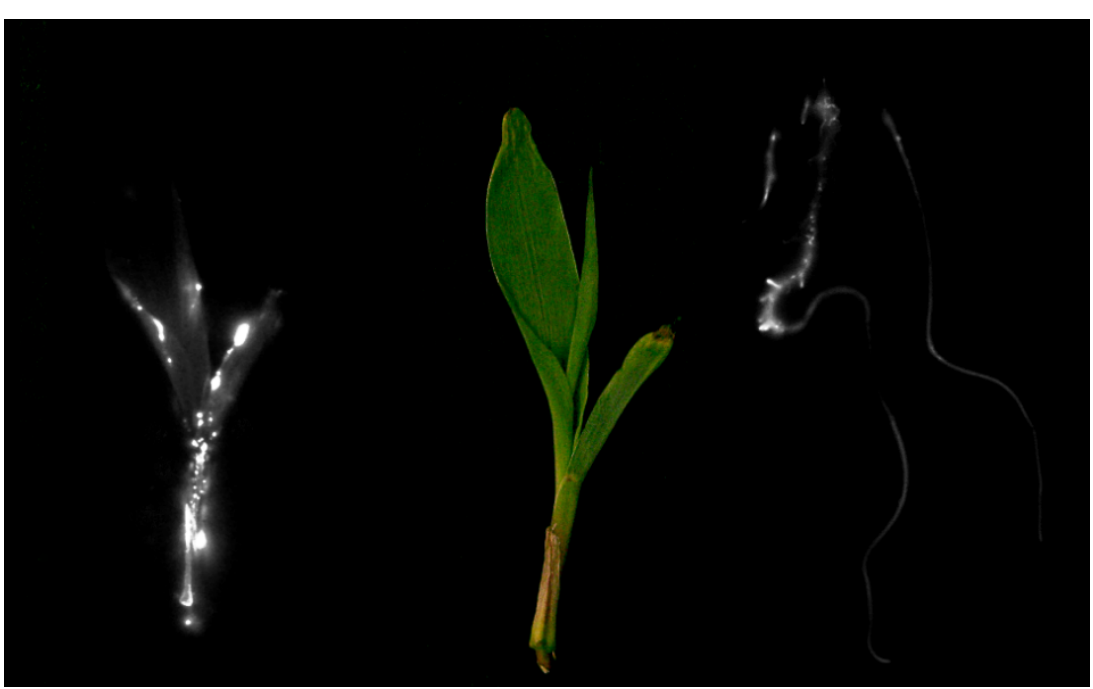

Figure 2.3.2.: Original images for composite image in Figure

2.3.1. B. The Figure in 2.3.1. B is a compositie photograph overlaying an autoradiographic image atop a digital photograph of the stem and leaf. The original, non-overlayed images, appear here. dioxide $\left(\left[{ }^{11} \mathrm{C}\right] \mathrm{CO}_{2},{ }^{11} \mathrm{C} \mathrm{t}_{1 / 2}=\right.$ $20.4 \mathrm{~min})$ were also administered to maize plants three weeks post germination (Figures 2.3.1. C-D and 2.3.3.). $\left[{ }^{18} \mathrm{~F}\right] \mathrm{FDG}$ is used as a glucose mimic and $\left[{ }^{11} \mathrm{C}\right] \mathrm{CO}_{2}$ can be used to image

$\left[{ }^{11} \mathrm{C}\right]$ photoassimilates ${ }^{36}$. Autoradiographic images by 
phosphor film imaging were done as previously described ${ }^{36}$.

Autoradiography is a static, twodimensional radio-imaging technique. Our instrument parameters were set at $100 \mu \mathrm{m}$ resolution.

The images obtained can provide information about static B localization patterns in root and shoot tissues. In

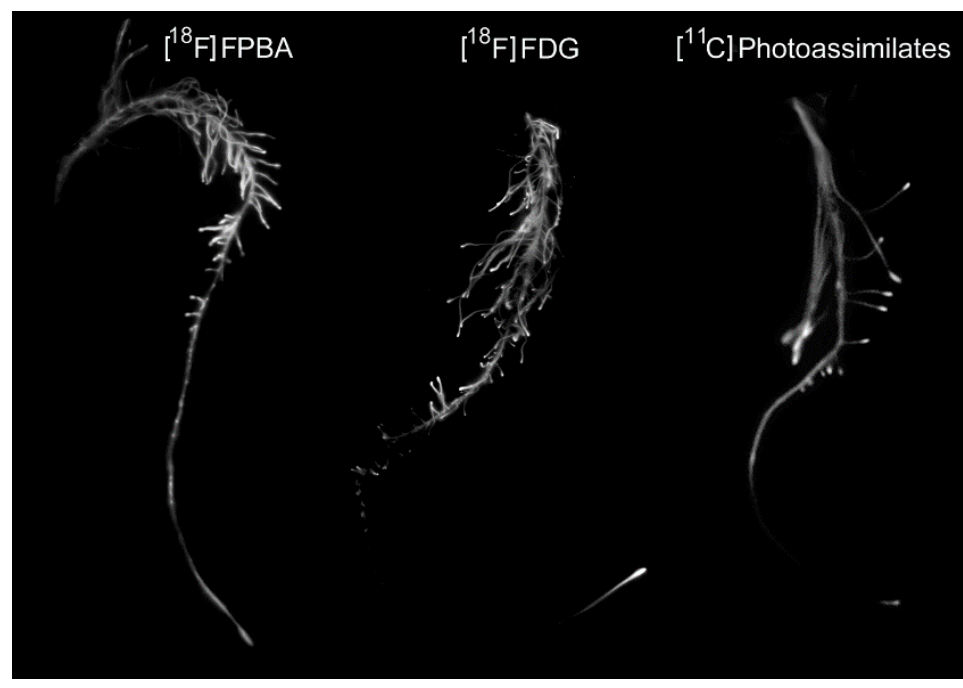

Figure 2.3.3.: Isolated root images, showing localization of $\left[{ }^{18} \mathrm{~F}\right] \mathrm{FPBA},\left[{ }^{18} \mathrm{~F}\right] \mathrm{FDG}$ and $\left[{ }^{11} \mathrm{C}\right]$ Photoassimilates in roots of three week old maize plants (inbred B73) as indicated.

the radiographic images, the tissues with higher concentration of radiotracer uptake appear more intensely white while areas of no uptake appear black. It is apparent from the radiographic images that tracer activity was taken up and localized heterogeneously across the maize roots with greater uptake within the primary root and lateral root initiation sites along the primary root (Figure 2.3.1. B-D, Figures 2.3.2. and 2.3.3.). This differs greatly from the homogenous uptake exhibited by the $\left[{ }^{18} \mathrm{~F}\right] \mathrm{FDG}$ tracer, which was also passively taken up by the roots. The $\left[{ }^{11} \mathrm{C}\right] \mathrm{CO}_{2}$ images resulting from passive tracer uptake through the leaf tissue show similar patterning of $\left[{ }^{11} \mathrm{C}\right]$-photosynthates in the tips of the primary roots (Figure 2.3.1. C-D, Figure 2.3.3.). The $\left[{ }^{18} \mathrm{~F}\right] \mathrm{FPBA}$ radiotracer additionally translocated into the shoot, with signals being detected in the leaf margins (Figures 2.3.1. B and 2.3.2.).

FPBA and PBA elicit similar phenotypic responses; Germination of maize kernels in PBA and FPBA- To enable the interpretation of the localization of $\left[{ }^{18} \mathrm{~F}\right] \mathrm{FPBA}$ in the radiotracer studies and since the effects of PBA on maize development have not been previously explored, we performed extensive testing of the effects of PBA and unlabeled FPBA on maize seedling 
development. We rolled maize kernels of the B73 inbred line into paper towels, incubated them in different concentrations of PBA solution $(0-5 \mathrm{mM})$ and allowed them to germinate for five days in the dark at $28^{\circ} \mathrm{C}$ (Figure 2.3.4.). In comparison to kernels that were germinated in
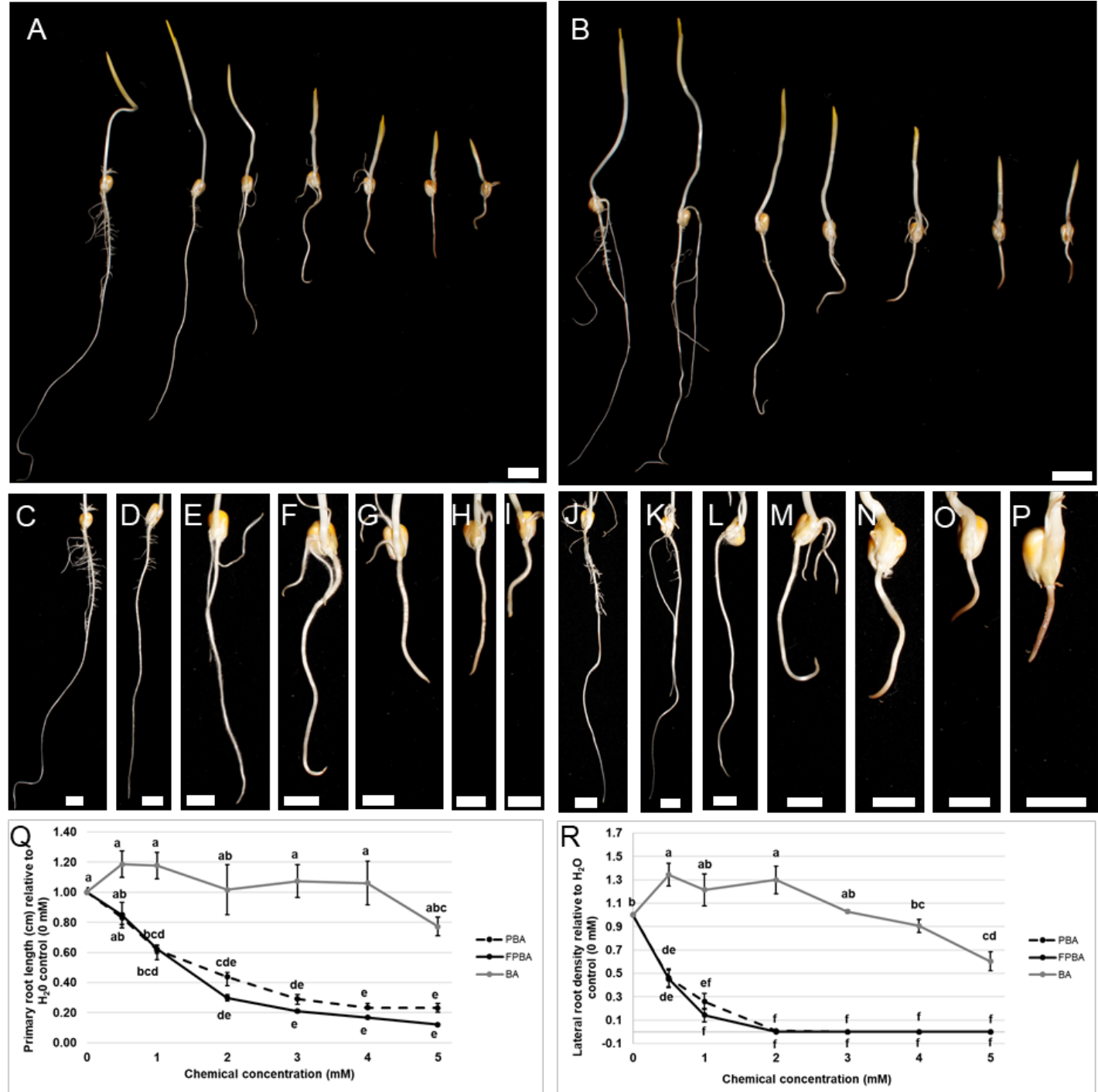

Figure 2.3.4.: Phenotypes of maize root growth (B73 inbred) germinating in different concentrations of PBA and FPBA. A) Maize B73 seedlings five days after germination in varying concentrations of PBA (from left to right: $\mathrm{H}_{2} \mathrm{O}, 0.5 \mathrm{mM}, 1 \mathrm{mM}, 2 \mathrm{mM}, 3 \mathrm{mM}, 4 \mathrm{mM}$ and $5 \mathrm{mM}$ ). B) Maize B73 seedlings five days after germination in varying concentrations of FPBA (from left to right: $\mathrm{H}_{2} \mathrm{O}, 0.5 \mathrm{mM}, 1 \mathrm{mM}, 2 \mathrm{mM}, 3 \mathrm{mM}, 4 \mathrm{mM}$ and $5 \mathrm{mM}$ ). C) - I) close up of B73 roots five days after germination in $\mathrm{H}_{2} \mathrm{O}(\mathrm{C}), 0.5 \mathrm{mM}$ PBA (D), $1 \mathrm{mM}$ PBA (E), 2 mM PBA (F), 3 mM PBA (G), 4 mM PBA (H) and 5 mM PBA (I). J) - P) Close up of B73 roots five days after germination in $\mathrm{H}_{2} \mathrm{O}(\mathrm{J}), 0.5 \mathrm{mM}$ FPBA $(\mathrm{K}), 1 \mathrm{mM}$ FPBA (L), $2 \mathrm{mM}$ FPBA $(\mathrm{M}), 3 \mathrm{mM}$ FPBA $(\mathrm{N}), 4 \mathrm{mM}$ FPBA (O) and $5 \mathrm{mM}$ FPBA (P). Q) Statistical analysis of primary root length of B73 seedlings grown in varying concentrations of PBA, FPBA or BA. R) Statistical analysis of lateral root density of B73 seedlings grown in varying concentrations of PBA, FPBA or BA. Different letters indicate statistical significance at $\mathrm{p}<0.05$ according to ANOVA and a posthoc Tukey test. Scale bar in $\mathrm{A}, \mathrm{B}=2 \mathrm{~cm}$ and in $\mathrm{C}-$ $\mathrm{P}=1 \mathrm{~cm}$. 
Millipore water, the PBA treated kernels developed into seedlings with shorter roots and

coleoptiles (Figure 2.3.4. C-I). We focused on the effects of PBA on root growth and found that the lowest concentration of PBA, which elicited a significant decrease in primary root length,

was $1 \mathrm{mM}$ (Figure 2.3.4. Q). The effect of F-PBA on maize root development was tested (0 - $5 \mathrm{mM}$ FPBA), as done for PBA.

After five days in the dark at $28^{\circ} \mathrm{C}$ a significant reduction was detected in primary root length in seedlings treated with FPBA compared to seedlings germinated in Millipore water, starting at 1 mM FPBA (Figure 2.3.4. J-P). The reduction in root growth with FPBA was not significantly different from PBA treated B73 kernels, as determined by analysis of variance (ANOVA) followed by a Tukey test for multiple testing correction (Figure 2.3.4. Q). Treatment with BA did not lead to a reduction in primary root length (Figure 2.3.5.), rather a slight increase in primary root length was observed when seedlings were germinated in either $0.5 \mathrm{mM}$ BA or $1 \mathrm{mM}$ BA. Higher
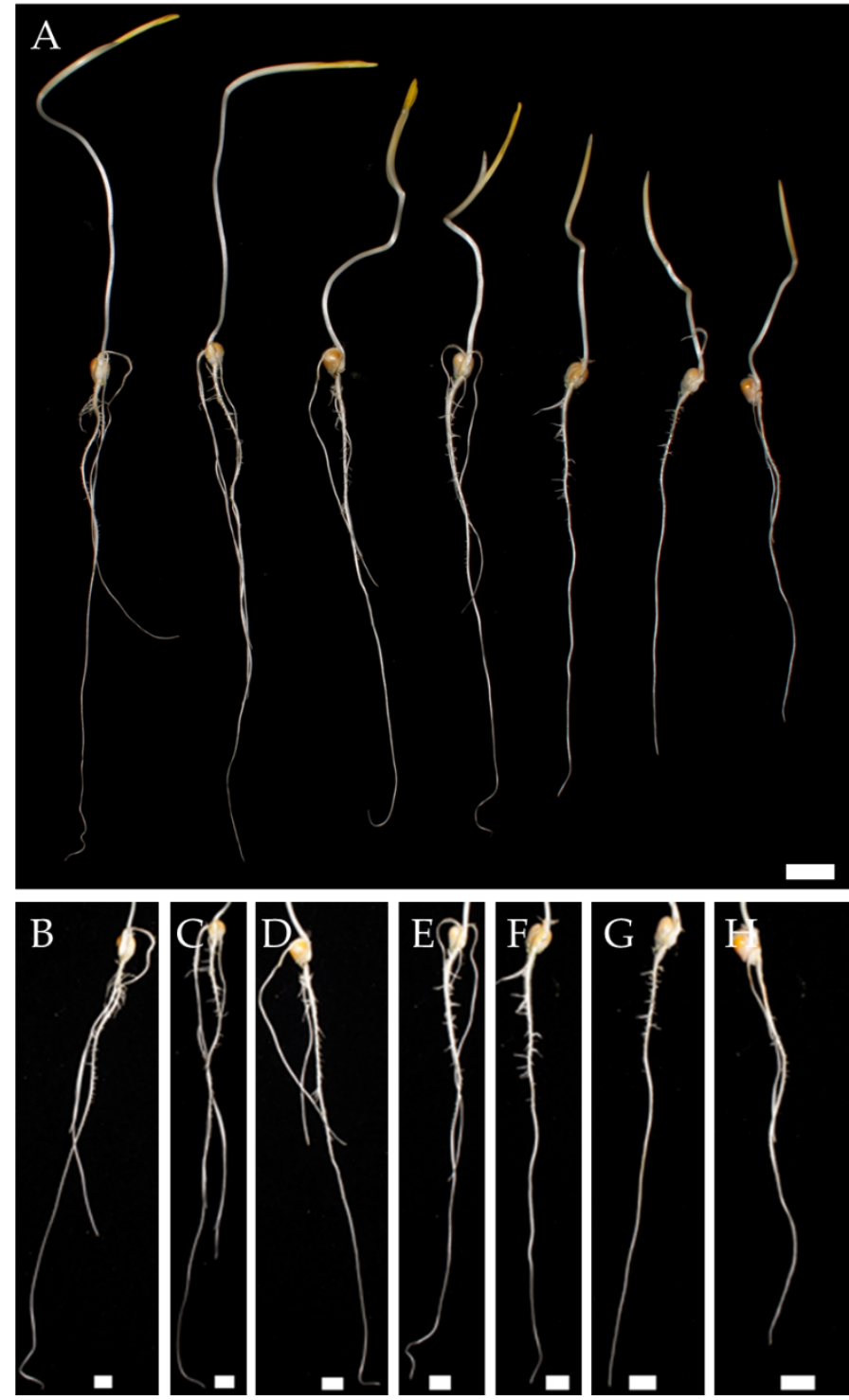

Figure 2.3.5.: Phenotypes of maize $B 73$ root growth germinating in different concentrations of $B A$. A) Maize B73 seedlings five days after germination in varying concentrations of $\mathrm{BA}$ (from left to right: $\mathrm{H} 2 \mathrm{O}$, $0.5 \mathrm{mM}, 1 \mathrm{mM}, 2 \mathrm{mM}, 3 \mathrm{mM}, 4 \mathrm{mM}$ and $5 \mathrm{mM}$ ). B) - H) close up of $\mathrm{B} 73$ roots 5 days after germination in $\mathrm{H} 2 \mathrm{O}$ (B), $0.5 \mathrm{mM}$ BA (C), $1 \mathrm{mM}$ BA (D), $2 \mathrm{mM}$ BA (E), 3 mM BA (F), $4 \mathrm{mM} \mathrm{BA}(\mathrm{G})$, and $5 \mathrm{mM} \mathrm{BA}(\mathrm{H})$. Scale bar in $\mathrm{A}=2 \mathrm{~cm}$, and $\mathrm{B}-\mathrm{H}=1 \mathrm{~cm}$. 
concentrations $(5 \mathrm{mM})$ led to a reduction in primary root length, but not to the extent PBA did (Figure 2.3.4. Q). Next, the occurrence and density of lateral roots in B73 seedlings that were germinated in different concentrations of PBA, FPBA and BA were analyzed (Figures 2.3.4.

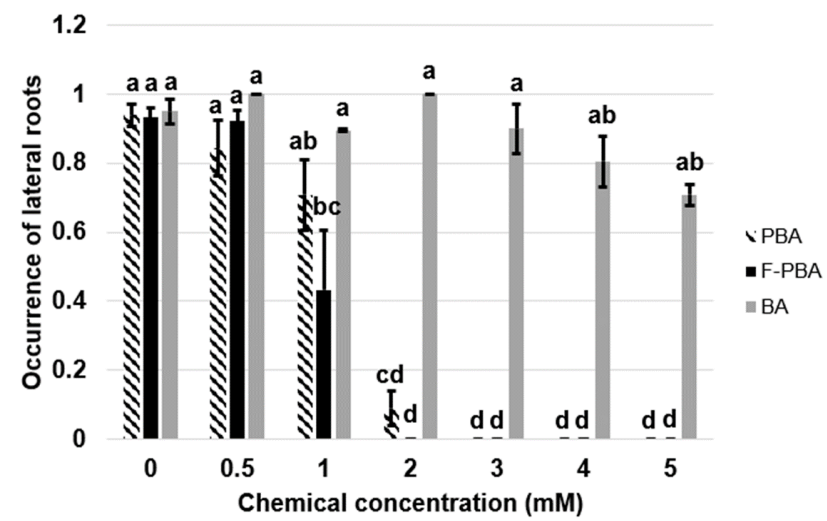

Figure 2.3.6.: Percentages of B73 maize seedlings with lateral roots five days after germination in different concentrations of PBA, FPBA and BA. Dissimilar letters indicate differences of $\mathrm{p}<0.05$ (ANOVA). and 2.3.6.). Concentrations of $2 \mathrm{mM}$ or higher of both FPBA and PBA led to a total absence of lateral roots, while the tested concentrations of BA $(0 \mathrm{mM}-5 \mathrm{mM})$ did not (Figure 2.3.6.). The occurrence of a "nude" root phenotype appeared with lower concentrations of FPBA than PBA (compare 1 $\mathrm{mM}$ to $2 \mathrm{mM}$ in Figure 2.3.6.). We measured

the area of the primary root where lateral roots occurred and counted all lateral roots that had developed to calculate the lateral root density per $\mathrm{cm}$. Similar to the effects on primary root length, PBA and FPBA elicited similar effects on lateral root density (Figure 2.3.4. R). Low concentrations $(0.5 \mathrm{mM})$ of either chemical led to a significant decrease in lateral roots and germination of B73 seedlings in $2 \mathrm{mM}$ PBA/FPBA or higher resulted in a lateral root density of zero (Figure 2.3.4. R). In comparison, low concentrations of BA $(0.5 \mathrm{mM}-2 \mathrm{mM})$ led to a slight, but significant increase in lateral root density (Figure 2.3.4. R). The highest concentration of BA used in this study ( $5 \mathrm{mM})$ led to a significant decrease in lateral root density, although it did not reach zero, like the PBA and FPBA treatment (Figure 2.3.4. R). FPBA induces rootless Arabidopsis seedlings- To further validate that PBA and FPBA elicit similar effects, we treated developing Arabidopsis siliques with 50 mM PBA and 50 mM FPBA. 
PBA was previously shown to induce

Arabidopsis seedlings with no primary $\operatorname{root}^{28}$.

FPBA treatment of developing siliques led to the

same rootless phenotype (Figure 2.3.7.).
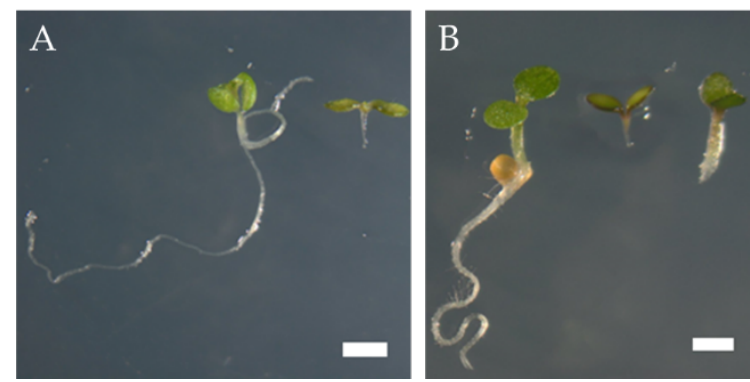

Mimicking of B deficiency symptoms by

Figure 2.3.7. : Phenotypes of seedlings resulting from PBA and FPBA treated Arabidopsis thaliana siliques. A) $50 \mathrm{mM}$ PBA

PBA/FPBA; Co-treatment of PBA/FPBA and

$B A$ decreases the PBA/FPBA induced (left: normal, right: $m p$ phenocopy). B) $50 \mathrm{mM}$ FPBA (left: normal, middle: $m p$ phenocopy, right: $b d l$ phenocopy). Scale bars $=1 \mathrm{~cm}$

inhibition of primary root length in maize- In order to assess whether the observed reductions in primary root lengths were due to B deficiency, we tested whether the PBA/FPBA induced inhibition of primary root length can be alleviated by BA. Since PBA/FPBA are hypothesized to
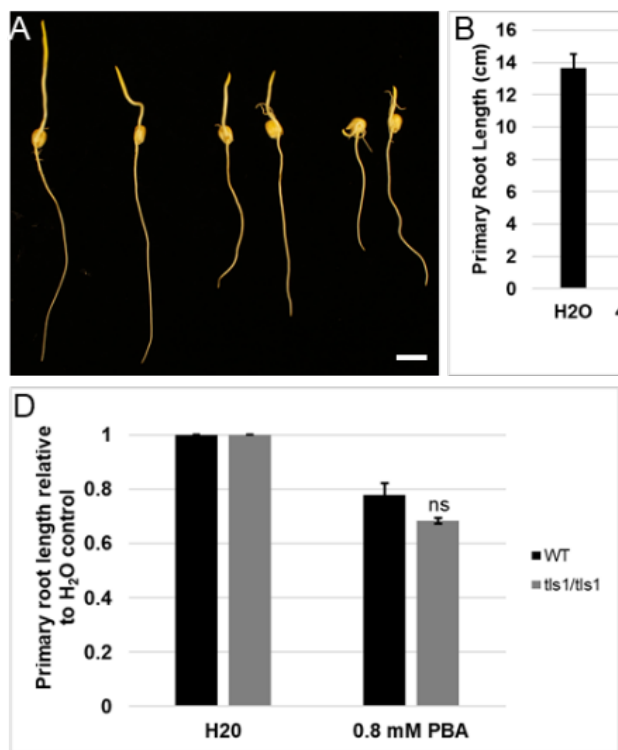
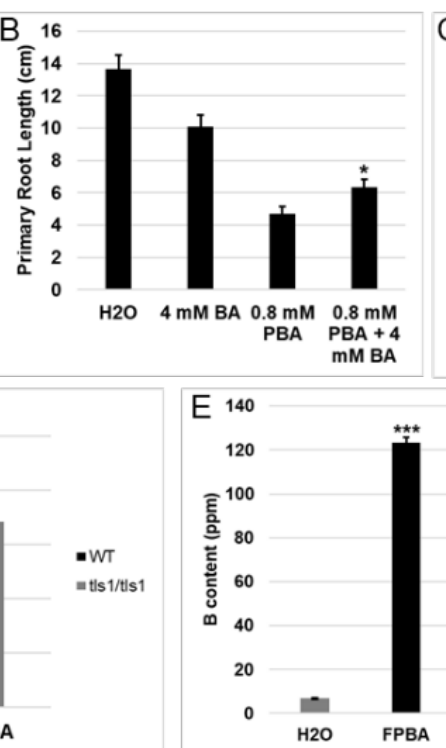
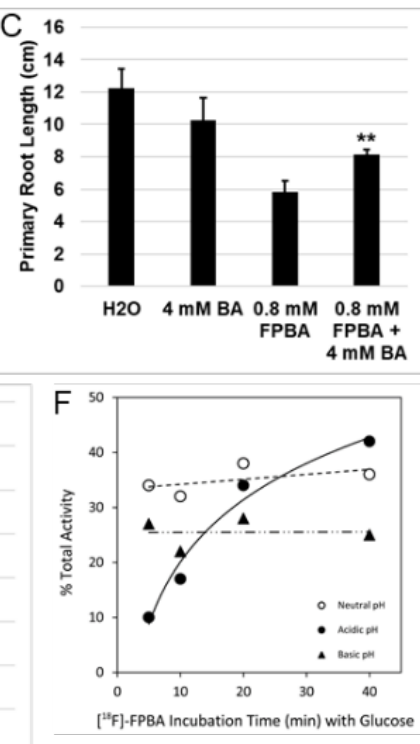

Figure 2.3.8 Mimicking of B deficiency symptoms by PBA/FPBA. A) Rescue of PBA/FPBA induced primary root length defects by BA. From left to right: Seedlings germinated for five days in $\mathrm{H}_{2} \mathrm{O}, 4 \mathrm{mM}$ BA, $0.8 \mathrm{mM}$ PBA, $0.8 \mathrm{mM}$ PBA $+4 \mathrm{mM}$ BA, $0.8 \mathrm{mM}$ FPBA, $0.8 \mathrm{mM}$ FPBA +4 mM BA. B) Statistical analysis of primary root length of the rescue of PBA induced primary root length defects by BA as exemplified in A). Shown is an individual experiment. C) Statistical analysis of primary root length of the rescue of FPBA induced primary root length defects by BA as exemplified in A). Shown is an individual experiment. D) Analysis of primary root lengths relative to $\mathrm{H}_{2} \mathrm{O}$ control for $Z m t l s 1$ and normal seedlings grown for five days in either $\mathrm{H}_{2} \mathrm{O}$ or $0.8 \mathrm{mM}$ PBA. E) B measurements via ICP-MS in roots of seedlings germinated for five days in either $\mathrm{H}_{2} \mathrm{O}$ or $2 \mathrm{mM}$ FPBA. F) Binding affinity of $\left[{ }^{18} \mathrm{~F}\right] \mathrm{FPBA}$ to glucose. Scale bar in A) $=2 \mathrm{~cm} .{ }^{*} \mathrm{p}<0.05,{ }^{*} \mathrm{p}<0.01$, and $* * * \mathrm{p}<0.001$ (Student's t-test). ns $=$ non significant. 


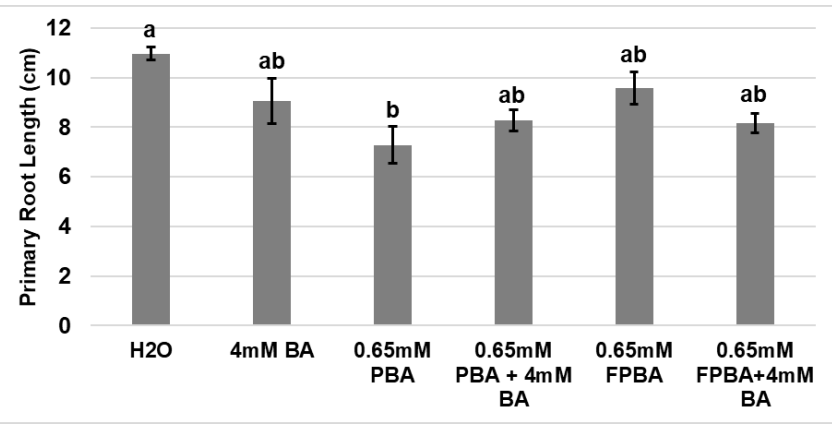

Figure 2.3.9. : Analysis of primary root lengths of $B 73$ maize seedlings germinated for five days in $\mathrm{H}_{2} \mathrm{O}, 4 \mathrm{mM}$ BA, $0.65 \mathrm{mM}$ PBA, $0.65 \mathrm{mM}$ PBA +4 mM BA, $0.65 \mathrm{mM}$ FPBA, and $0.65 \mathrm{mM} \mathrm{FPBA}+4$ $\mathrm{mM}$ BA. Dissimilar letters indicate differences of $\mathrm{p}$ $<0.05$ (ANOVA). compete with BA for its binding sites, it was predicted that higher concentrations of BA in comparison to $\mathrm{PBA} / \mathrm{FPBA}$ concentrations would be needed to rescue root growth. Therefore, B73 kernels in $0.8 \mathrm{mM}$ PBA/FPBA were co-incubated with $4 \mathrm{mM}$ BA (Figure 2.3.8. and 2.3.9.) and dark germinated for five days at $28^{\circ} \mathrm{C}$. Treatment

with these concentrations of PBA and FPBA led to signficantly shorter primary roots in comparison to the $\mathrm{H}_{2} \mathrm{O}$ treatment (Figure 2.3.9.). In the individual experiments, co-treatments with BA led to significantly longer primary roots in comparison to PBA or FPBA treatment alone according to Student's t- test (Figure 2.3.8. A-C). A combined analysis still led to slightly longer primary roots, yet no statistical significance was detected (Figure 2.3.9.).

The B deficient mutants Zmtls1 and Zmrte are slightly more sensitive to PBA treatment compared to their normal siblings- We next tested the sensitivity of the inherently B deficient

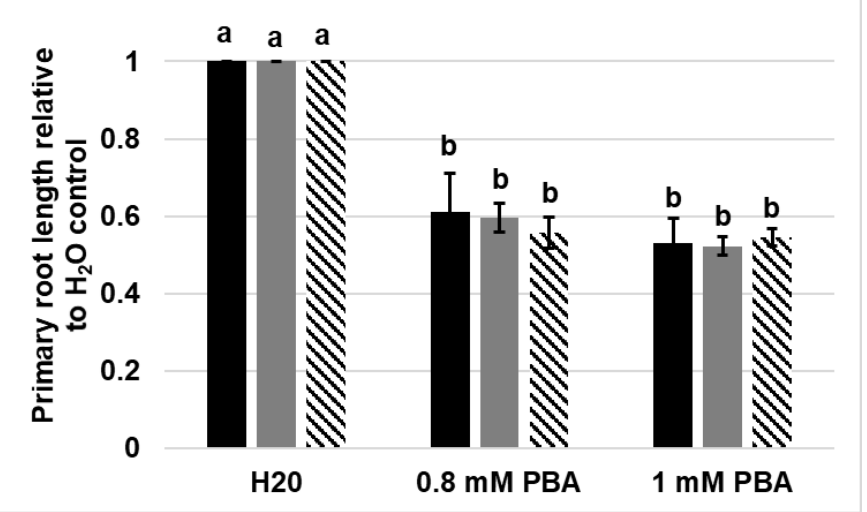

Figure 2.3.10.: Primary root length relative to water control of WT, rte/+, and rte/rte maize seedlings that were germinated in different concentrations of PBA. Dissimilar letters indicate differences of $\mathrm{p}<0.05$ (ANOVA). mutants Zmtls1 and Zmrte to PBA, by germinating mutant segregating lines in the B73 genetic background in $0.8 \mathrm{mM}$ PBA

(Figures 2.3.8. D and 2.3.10.). Both mutants were previously shown to have reduced cytosolic B levels ${ }^{23-24}$. Zmtls1 was also shown to have reduced RG-II crosslinking in the cell wall ${ }^{23}$. PBA $(0.8$ 
$\mathrm{mM}$ ) led to a significant decrease of primary root length in both normal and mutant siblings compared to the $\mathrm{H}_{2} \mathrm{O}$ control (Figures 2.3.8. D and 2.3.10.). PBA treatment $(0.8 \mathrm{mM})$ led to a slightly stronger, yet not significant, reduction of primary root length in both Zmtls 1 and Zmrte mutants compared to their normal siblings (Figures 2.3.8. D and 2.3.10.).

FPBA treatment causes enhanced cellular B levels in roots- PBA has previously been shown to deliver B to the cell in Arabidopsis ${ }^{37}$ and the induction of B deficiency symptoms is based upon PBA's interference with cell wall crosslinking. In order to test whether FPBA behaves in the same way regarding B delivery to cells, we quantified B levels using ICP-MS in five days old roots of seedlings germinated from kernels which were treated with $2 \mathrm{mM} \mathrm{F-PBA}$ or $\mathrm{H}_{2} \mathrm{O}$

(Figure 2.3.8. E). The amount of B measured was the summation of the concentration of both ${ }^{10} \mathrm{~B}$ and ${ }^{11} \mathrm{~B}$ isotopes naturally abundant. We found that B levels in FPBA treated roots were about 18 times higher compared to the $\mathrm{H}_{2} \mathrm{O}$ control (Figure 2.3.8. E). Other elements measured alongside $\mathrm{B}$ during the analysis (Figure 2.3.11.) showed no significant differences in concentration between treatments with the exception of

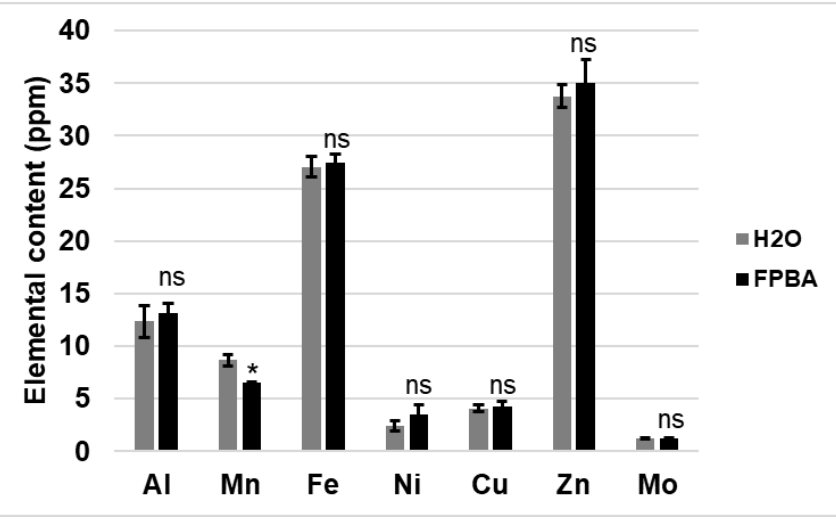

Figure 2.3.11.: ICP-MS analysis of different elemental contents in roots of five days old maize seedlings (B73 inbred), that were germinated in FPBA or H, O. * indicates $\mathrm{p}<0.05$ (Student's t-test). manganese, which showed significant reduction in the FPBA treated roots compared to $\mathrm{H}_{2} \mathrm{O}$ control roots.

$\left[{ }^{18} \mathrm{~F}\right]$ FPBA binds cis-diol groups- To test the prediction that $\left[{ }^{18} \mathrm{~F}\right] \mathrm{FPBA}$, like PBA and $\mathrm{BA}{ }^{26,38}$, has high affinity for covalent bonding with cis-diol groups, we performed an experiment to test binding affinity with glucose which is a proposed binding site in vivo ${ }^{38}$. If a tracer is designed to 
mimic $\mathrm{B}$ behavior, it must bind cis-diols. Binding affinity was assessed with introduction of a cis-diol to the tracer at biologically relevant $\mathrm{pH}$ and separation of bound and unbound tracer was measured via radio-Thin Layer Chromatography (radio-TLC). Glucose naturally exists in various physical conformations consisting in a linear structure, a six-membered ring pyranose structure and a five-membered ring furanose structure, with the most common being pyranose in aqueous solutions. The furanose conformations of glucose also exist at low percentages and would have the strongest binding affinity for the $\left[{ }^{18} \mathrm{~F}\right] \mathrm{FPBA}$ based on the angles of the cis-diol bonds. The $\mathrm{pH}$ conditions can have an effect on the relative prevalence of the glucose conformers. Our data shows that as $\mathrm{pH}$ decreases, the total percent of bound radiotracer increases over time, while the neutral and basic $\mathrm{pH}$ show greater initial binding, with no enhancement of tracer binding over time (Figure 2.3.8. F).

\subsection{Discussion}

B deficiency occurs in soils worldwide ${ }^{4}$ and has negative effects on plant growth and performance. The underlying molecular mechanisms of how B deficiency limits plant growth are only marginally understood, in particular because of the limited availabilities of B imaging techniques. Here, we report the development of a B radiotracer in maize that is based upon the boronic acid FPBA. Boronic acids, like PBA, have been implicated in inducing B deficiency symptoms ${ }^{26,28}$. To our knowledge, this is the first report of assessing the usability of a B deficiency mimic as a B radiotracer in plants and for assessing the effects of PBA on maize root development.

$\mathrm{B}$ belongs to a group of elements that does not possess any radioactive isotope with a $t_{1 / 2}$ appropriate for isotopic labelling and autoradiography, since the longest $\mathrm{t}_{1 / 2}$ belongs to ${ }^{8} \mathrm{~B}$ at 770 $\mathrm{ms}^{34}$. A similar restriction is seen for $\mathrm{O}$ (longest $\mathrm{t}_{1 / 2}=2 \mathrm{~min}$ ). Because of these challenges in the 
possibility of labelling BA (the plant accessible form of B), we explored ways of labelling the B deficiency mimic PBA instead.

The most suitable radioisotopes for imaging an organic-based small molecule, like PBA, are those found before atomic number 10 (neon) on the periodic table ${ }^{39}$. Radioisotopes before carbon often have a $t / 2$ which is too short for synthesis, purification, and detection, while moving beyond into larger elements begins changing the chemical and physical properties of the small molecule. Such changes challenge the usefulness as a tracer of genuine chemical-of-interest behavior ${ }^{40}$. This makes ${ }^{18} \mathrm{~F}$ an attractive radioisotope for radiolabeling PBA.

Fluorine is more electronegative than the hydrogen it is replacing in PBA, but this is advantageous because the binding of BA and PBA in vivo is a result of deprotonation of the acidic hydrogens on the BA group for binding to cis-diols. Decreasing the $\mathrm{pK}_{\mathrm{a}}$ of the $\mathrm{BA}$ group increases the binding affinity of the tracer for its substrate ${ }^{41}$.

The radiotracer structure is not novel, as the replacement of a BA group with ${ }^{18} \mathrm{~F}$ on benzene rings has been an important aspect in medical research ${ }^{42}$. The radiotracer synthesis method described in this work is an automated version of a previously described manual synthesis ${ }^{35}$. Automated synthesis enhances safety for personnel and increases the level of radioactivity usable in the synthesis. Removal of the copper catalyst with a Chelex ${ }^{\circledR}$ column and slight alterations to reagents and reaction times were also made.

The unique aspect of the radiotracer primarily lies in its application here for B imaging in live plant systems, which has not been previously explored. This radiotracer has advantages of a streamlined synthesis and hydroponic passive administration to plant ${ }^{5}$. This allows avoidance of plant-stress responses that might otherwise have an impact on the observations and data. The stability and binding studies show $\left[{ }^{18} \mathrm{~F}\right] \mathrm{FPBA}$ has appreciable binding to the $c i s$-diols like 
glucose across various $\mathrm{pH}$ values (Figure 2.3.8. F) which means it will be able to bind to substrates across the entire spectrum of expected $\mathrm{pH}$ values in living plant systems and remain stable.

Autoradiography allows imaging of the localization of BA binding sites under the assumption that the radiotracer is intact and validly behaves just as BA in plant systems. The binding affinity and stability studies corroborate tracer physical integrity in vivo and the phenotypic studies suggest PBA behavior of the FPBA molecule in maize. The images can be overlaid on visual photographs to make more definitive conclusions concerning localization of the radiotracer and proposed purpose of higher B levels in such locations (Figures 2.3.1. B and

\subsection{2.).}

This $\left[{ }^{18} \mathrm{~F}\right] \mathrm{FPBA}$ tracer shows different imaging locations and distribution from typical $\left[{ }^{18} \mathrm{~F}\right] \mathrm{FDG}$ tracers especially along the elongation zone of the primary and lateral roots, which indicates binding specificity of the tracer (Figures 2.3.1. B-D, 2.3.2. and 2.3.3.). In five-daysold maize seedlings (Figures 2.3.1. B and 2.3.2.) the $\left[{ }^{18} \mathrm{~F}\right] \mathrm{FPBA}$ radiotracer localized primarily to lateral roots and to specific locations in the young leaves, while uptake in three-weeks-old maize plants was mostly restricted to the root system (Figures 2.3.1. C,D and 2.3.3.). The root tip and elongation zone displayed the most intense signal of the tracer with a region in between the two devoid of any signal (Figure 2.3.1. D Numbers 1-3). This particular pattern was also observed in the $\left[{ }^{11} \mathrm{C}\right]$-photoassimilate tracer (Figure 2.3.1. D Numbers 7-9) and suggests that the location of B binding sites and areas of high metabolism and carbon at least partially overlap. PBA was previously used for the design of a fluorescent marker and used to visualize B binding sites in maize and sunflower roots ${ }^{43}$. In this study by Gluesenkamp et al. in 1997, the PBA marker localized to the elongation zone and spared the meristematic zone at the root tip, which 
for the authors was in line with previous reports stating only expanding tissues have a high demand for $\mathrm{B}^{38}$. It is known that root meristem activity is inhibited under $\mathrm{B}$ deficiency ${ }^{44}$, suggesting a need for B in meristematic areas. The differences in localization between our tracer and the reported fluorescent marker are therefore likely due to structural differences of the two molecules. In five-days-old seedlings the $\left[{ }^{18} \mathrm{~F}\right] \mathrm{FPBA}$ radiotracer was additionally detected in the shoot at the edges of the leaves (Figures 2.3.1. B and 2.3.2.). This pattern is reminiscent of patterns of B accumulation, since excess B typically accumulates at the leaf edges as well ${ }^{45}$, therefore indicating that $\left[{ }^{18} \mathrm{~F}\right] \mathrm{FPBA}$ can be used to visualize sites of B occurrence in plants.

One of the fastest reported responses upon B deficiency is the cessation of root elongation in both the primary and the lateral roots ${ }^{12}$. When maize B73 kernels were germinated in PBA or FPBA a significant reduction of primary root length and a significant reduction of lateral root density was observed in comparison to kernels that were germinated in $\mathrm{H}_{2} \mathrm{O}$ (Figure 2.3.4.). The non-significant difference between PBA and FPBA treated seedlings indicates that the effects of PBA and FPBA on maize root development are comparable. This conclusion is also supported by an Arabidopsis experiment, where treatment of developing siliques with either PBA or FPBA leads to rootless Arabidopsis seedlings (Figure 2.3.7.). Browning of the root tip and swelling of the roots was also observed, particularly with higher concentrations of either chemical used (Figure 2.3.4. C-P). These findings are in good agreement with reported B deficiency symptoms in plant roots ${ }^{12}$, suggesting that PBA and FPBA can also be used in maize to induce B deficiency symptoms as reported for other species ${ }^{26}$. On the contrary, germination of B73 maize kernels in BA led to the opposite effects, namely slightly longer primary roots and an increase in lateral root density (Figures 2.3.4. Q-R and 2.3.5.). It further did not lead to a loss of lateral roots in any of the tested concentrations (Figure 2.3.6.). 
In all experiments FPBA was found to be slightly more potent in inducing phenotypic defects compared to PBA. Primary root length in B73 seedlings was shorter with lower concentrations of FPBA compared to PBA (Figure 2.3.4. Q), a "nude" root phenotype occurred with lower concentrations of FPBA (Figures 2.3.4. $\mathbf{R}$ and 2.3.6.), and the treatment of Arabidopsis embryos with FPBA led to two distinct phenotypes, suggesting either an earlier or prolonged effect of FPBA or slightly different targets of FPBA compared to PBA (Figure 2.3.7.). Although there are exceptions, it is generally assumed that boronic acids with lower $\mathrm{pK}_{\mathrm{a}} \mathrm{s}$ bind cis-diols more strongly. It is reasonable to speculate that the observed differences between PBA and FPBA $\left(\mathrm{pK}_{\mathrm{a}} \mathrm{s}\right.$ of 8.8 and 8.6 respectively ${ }^{46}$ ) are related to differences in their binding strengths such that FPBA binds cis-diols more strongly compared to PBA.

High concentrations of BA are toxic to plants and can lead to similar phenotypes to those observed under B deficiency. For example, excessive B has been reported to inhibit root growth in Arabidopsis ${ }^{47}$. While germinating B73 maize kernels in BA we also observed a reduction of primary root length in the highest BA concentrations likely because of toxicity. We measured via ICP-MS an 18-fold increase in B content in FPBA-treated roots compared to $\mathrm{H}_{2} \mathrm{O}$ control roots (Figure 2.3.8. E), indicating that FPBA is taken up by the roots. Other elements measured were not significantly different (Figure 2.3.11.). An exception to this was manganese, which showed significant reduction in FPBA-treated roots compared to the $\mathrm{H}_{2} \mathrm{O}$ control (Figure 2.3.11). The reason for this observation remains the subject of future analysis.

Because of the increase in B level, the observed reduction in primary root length and the browning of the tissue in PBA and FPBA treatments could be due to toxic cytosolic B contents. However, our data suggest that the reduction of primary root length caused by FPBA/PBA is due to B deficiency rather than toxicity, because: 1) Addition of BA (4 mM) partially restored the 
PBA-induced $(0.8 \mathrm{mM})$ reduction of primary root length (Figures 2.3.8. A-C and 2.3.9.), and 2) the maize mutants $t l s l$ and rte appeared to be slightly more sensitive to PBA treatment (Figures 2.3.8. D and 2.3.10.). The fact that addition of BA did not lead to a more severe reduction of primary root lengths in B73 seedlings makes it unlikely that the initial defects observed with PBA treatment are due to B toxicity, since the addition of BA would cause an even higher toxicity effect. The partial rescue in contrast of a full rescue by co-incubation of PBA with BA can likely be explained by the higher binding strength of PBA in comparison to BA because of their $\mathrm{pK}_{\mathrm{a}}$ values ${ }^{46}$, which will shift the odds for competitive binding in favor of PBA. One could hypothesize that once PBA binds a B- binding site within the cell wall, BA's lower binding strength cannot equally compete with PBA and the binding site is "lost" for BA. This scenario also explains why only high amounts of BA can partially rescue B deficiency defects induced by PBA as some available binding sites would be bound by BA. At the same time, BA concentrations cannot be increased infinitely as further increase in BA concentrations would lead to toxicity symptoms. This hypothesis is supported by previous studies with PBA, where PBAinduced defects were only partially or not at all rescued with added BA ${ }^{26,28}$.

The responsivity to PBA is variable between different biological replicates (seed batches). Although the duration of the treatments was constant between the experiments, actual root lengths were variable between the individual experiments. Because of this variability the partial rescue of the PBA and FPBA induced primary root length defects by BA could reach statistical significance only by direct comparison (Figure 2.3.8. B-C), but not when corrected for multiple testing (Figure 2.3.9.).

In a second line of experiments to assess the induction of B deficiency in maize seedlings by PBA, inherently B-deficient maize mutants $t l s l$ and $r t e$ both appeared slightly more sensitive to 
PBA treatment in comparison to their respective normal siblings (Figures 2.3.8. D and 2.3.10.) and displayed a higher reduction of primary root lengths when incubated in lower concentrations of PBA compared to their normal siblings. Zmtls1 is a mutant of a B importer gene, coorthologous to the Arabidopsis NIP5;1 B importer ${ }^{21}$, and Zmrte is co-orthologous to the Arabidopsis BOR1 B exporter ${ }^{20}$. Zmtls 1 completely lacks the $t l s 1$ gene, but can be rescued by addition of BA because of passive B transport ${ }^{23,48}$. The slight hypersensitivity of $Z m t l s 1$ and Zmrte to PBA suggests that PBA likely is transported passively. Direct transport of PBA by TLS1 or RTE cannot be excluded and will need to be assessed in the future by, for example, oocyte transport assays ${ }^{49}$. The reported role of PBA in interfering with cell wall crosslinking could be a cause of the enhanced sensitivity of Zmtls1 to PBA, as Zmtls1 was shown to have a reduced RG-II-B crosslinking, increased RG-II monomers, and reduced cellular B content ${ }^{23}$, which tempt the following speculations: 1) Less PBA is needed to occupy the remaining Bbinding sites; and 2) the reduced cellular B content in Zmtls 1 cannot successfully compete with PBA. Similar speculations can be drawn for Zmrte, although reduced RG-II-B crosslinking or altered RG-II monomers have not been shown in this mutant ${ }^{24}$. It is interesting to note that the responsivity of PBA as detected by a reduction in primary root lengths was different in Zmtls 1 compared to Zmrte lines (comparing Figures 2.3.8. D and 2.3.10.). PBA $(0.8 \mathrm{mM})$ led to an approximately $40 \%$ reduction of primary root lengths in normal siblings in Zmrte segregating lines (Figure 2.3.10), which is comparable to the observations with B73 (Figure 2.3.9.). The same concentration of PBA led to an approximately $23 \%$ reduction in primary root length in Zmtls1 segregating lines (Figure 2.3.8. D). Both mutants originated in different backgrounds than B73, yet have been backcrossed into B73 several times ${ }^{23-24}$. It is likely that remnants of the original backgrounds, specifically for Zmtls1 segregating lines cause the difference in phenotypic 
expression. Further research is needed to elucidate and confirm underlying causes for the displayed hypersensitivity and variation between genotypes, which is beyond the scope of this report.

In conclusion, we report the development a $\left[{ }^{18} \mathrm{~F}\right] \mathrm{FPBA} \mathrm{B}$ radiotracer to image $\mathrm{B}$ binding sites in maize. In good agreement with previous reports, our tracer localizes to particularly the tip, the elongation zone, and lateral roots in the maize root, showing a demand for B in these regions. Follow up phenotypic analyses with PBA and unlabeled FPBA suggest that the observed root defects are caused by B deficiency symptoms, likely because of an interference in Bdependent cell wall crosslinking. Our phenotypic data therefore support the usability of $\left[{ }^{18} \mathrm{~F}\right] \mathrm{FPBA}$ to image $\mathrm{B}$ binding sites in planta.

\subsection{Materials and Methods}

Fluorine-18 was obtained in Oxygen-18 enriched water from a $16.4 \mathrm{MeV}$ biomedical cyclotron (GE PETrace) with a starting activity of approximately $300 \mathrm{mCi}$. All chemistry was done remotely in a radiation safety hood. Activity was collected on a Waters Sep-Pak ${ }^{\mathrm{TM}}$ Light Accell Plus QMA Carbonate Cartridge (Milford, MA, USA) and eluted from the cartridge with $0.55 \mathrm{~mL}$ of a $0.05 \mathrm{M}$ potassium trifluoromethanesulfonate (Aldrich, St. Louis, MO, USA) and $1.3 \mathrm{mM}$ potassium carbonate solution. The eluted ${ }^{18} \mathrm{~F}^{-}$was dried repeatedly with acetonitrile (Fisher, Hampton, NH, USA) $0.5 \mathrm{~mL}$ at a time at $100{ }^{\circ} \mathrm{C}$ in a reaction vessel suspended in an oil bath under argon gas. Dimethylformamide (Acros Organics) was added separately to $6.63 \mathrm{mg}$ of benzene 1,4-diboronic acid (Oakwood Chemicals, Fair Lawn, NJ, USA), $0.810 \mathrm{~mL}$ pyridine (Acros Organics, Fair Lawn, NJ, USA), and $72.3 \mathrm{mg}$ of copper (II) trifluoromethanesulfonate (Alfa Aesar, Haverhill, MA, USA). Once dissolved, $0.5 \mathrm{~mL}$ of the pyridine mixture, $0.10 \mathrm{~mL}$ of the copper mixture, and $0.05 \mathrm{~mL}$ of the benzene mixture were mixed together and this precursor solution was injected into the reaction vessel containing the dried residue of the fluorine solution. 
This solution was reacted at $110{ }^{\circ} \mathrm{C}$ for $10 \mathrm{~min}$ and mixed occasionally to form the unpurified $\left[{ }^{18} \mathrm{~F}\right]$ FPBA. This solution was allowed to cool, then transferred through a SPE cartridge body containing $1 \mathrm{~g}$ of Chelex ${ }^{\circledR} 100$ resin (100-200 mesh, sodium form, Bio-Rad Laboratories, Hercules, CA) held in place by a glass wool packing. This step was performed in an attempt to remove excess copper from the solution. The $\left[{ }^{18} \mathrm{~F}\right] \mathrm{FPBA}$ was rinsed off the resin and glass wool with $1 \mathrm{~mL}$ of 50:50 methanol:0.5 $\mathrm{M} \mathrm{HCl}$ and collected in a vial upon elution.

The $\left[{ }^{18} \mathrm{~F}\right]$ FPBA product was purified on a semi-prep Phenomenex Luna $5 \mu \mathrm{m}$ column (250 x10 mm, 100 A. PN:DN-00G-4448-N0) with a mobile phase of 70:30 acetonitrile:water plus $0.1 \%$ trifluoroacetic acid at $4 \mathrm{~mL} / \mathrm{min}$, and the UV-detector set to a $254 \mathrm{~nm}$ wavelength. The retention time of the product is approximately $15 \mathrm{~min}$. Upon detection by the UV and radiation detectors the product was collected in $30 \mathrm{~mL}$ of water. It was then applied to a Waters Oasis HLB light $30 \mathrm{mg}$ Sep-Pak ${ }^{\mathrm{TM}}$ cartridge for solvent removal and concentration. This cartridge was eluted with $1 \mathrm{~mL}$ of ethanol and evaporated to dryness with gentle heat argon. The product $\left[{ }^{18} \mathrm{~F}\right] \mathrm{FPBA}$ was then reconstituted first in $1 \mathrm{~mL}$ of water and a sample was removed for quality control testing.

Quality control of the radiotracer involved analytical HPLC as well as radio-TLC analysis of the final product. Upon resuspension of the radiotracer in $1 \mathrm{~mL}$ of water, $10 \mu \mathrm{L}$ was injected onto the HPLC and analyzed on a Luna $5 \mu$ PFP(2) 100 A analytical column $(2.50 \times 4.6 \mathrm{~mm}$, PN: 00G-4448-E0) and detected by radioactivity detector and internal UV detector. The mobile phase was as per preparative, flowing at $1 \mathrm{~mL} \mathrm{~min}{ }^{-1}$. The final product spectra was compared to a cold FPBA standard and a phenyldiboronic acid standard. Percent labeled radiotracer was measured with radio-TLC on $3 \times 10 \mathrm{~cm}$ amino-backed silica plates developed in a chamber of 2:1 methylene chloride:methanol mobile phase. Once developed, the distribution of activity was 
measured by a Bioscan AR-2000 radio-TLC reader and WinScan software. The spots where identified by a comparison of their location to a cold plate run with cold precursor and product standards.

After resuspension of the dried radiotracer in $1 \mathrm{~mL}$ of water, it was further diluted to $10 \mathrm{~mL}$ of DI water for the five-day old maize or $20 \mathrm{~mL}$ for the three-week old maize and swirled to adequately mix. Approximately $200 \mu \mathrm{Ci}$ of tracer was then hydroponically administered to the root system of the maize plants either at five days or three weeks post-germination by suspending the plant roots in the tracer-water. Tracer uptake was enhanced by illumination of the plant with LED lights to drive transpiration and water uptake. The plant and beaker were placed within a Plexiglass ${ }^{\mathrm{TM}}$ and lead lined radiotracer stall, held under slight negative pressure for safety. The plants were incubated with radiotracer for approximately $90 \mathrm{~min}$ before removal and autoradiography.

Tracer affinity for glucose across different $\mathrm{pH}$ levels was tested using the tracer made as previously described. Once eluted off the HLB Sep-Pak ${ }^{\mathrm{TM}}$, the $1 \mathrm{~mL}$ of ethanol tracer was dried under gentle heating and argon gas and re-suspended in $3 \mathrm{~mL}$ of DI water. Three separate 1-mL aliquots were taken and acidic, neutral, and basic $10 \mathrm{mM}$ glucose solutions were added to the tracer water aliquots, respectively. The neutral glucose solution was $3 \mathrm{~mL}$ total volume with no additions and was a $\mathrm{pH}$ of 6.4 . The acidic glucose was made by taking another $3 \mathrm{~mL}$ of $10 \mathrm{mM}$ glucose and adding $30 \mu \mathrm{L}$ of $0.03 \mathrm{M} \mathrm{HCl}$, which was at a $\mathrm{pH}$ of 4 . The basic glucose solution was made with a 3-mL aliquot of $10 \mathrm{mM}$ glucose with $30 \mu \mathrm{L}$ of $0.027 \mathrm{M} \mathrm{NaOH}$, bringing the $\mathrm{pH}$ to 9. Once the three glucose solutions were made and mixed with the radiotracer, samples were taken at time points of 0,10 , and $20 \mathrm{~min}$ and percent bound tracer was investigated with radioTLC. The three $\mathrm{pH}$ glucose-tracer solutions were spotted onto amino-backed silica plates at the 
origin and developed in 2:1 methylene chloride:methanol until complete when radio-TLC analysis was completed to determine percent bound tracer to glucose across $\mathrm{pH}$ and time.

Tracer administration for the $\left[{ }^{18} \mathrm{~F}\right] \mathrm{FDG}$ comparison trials $(500 \mu \mathrm{Ci})$ was accomplished in an identical manner as the $\left[{ }^{18} \mathrm{~F}\right] \mathrm{FPBA}$, but the administration for the $\left[{ }^{11} \mathrm{C}\right]$-photosynthate trials were different. The $\left[{ }^{11} \mathrm{C}\right] \mathrm{CO}_{2}$ was made on the cyclotron and administered to the maize seedling $(\sim 20$ $\mathrm{mCi}$ ) where it was passively administered in a gaseous bolus to a load leaf. This load leaf was placed within an air-tight gasketed cuvette in which the $\left[{ }^{11} \mathrm{C}^{-C_{2}}\right.$ flowed in and out. After administration, the plant was allowed to metabolize the radiotracer for approximately $90 \mathrm{~min}$ at which point the plant was removed for imaging as done in previous work ${ }^{36}$.

Once removed from the radiotracer stall, and from either the cuvette or the beaker of aqueous radiotracer, the plant was patted dry and laid atop a phosphor imaging film. A visual (digital image) was taken of the plant prior to exposure to the film for overlay of the images later in Adobe Photoshop software. Original images prior to overlay can be found in the supplemental information (Figure 2.3.2.). The plate was exposed overnight $(\sim 15 \mathrm{~h})$, and then read on a Typhoon 9000 imager the following morning, imaging of the roots separately from the leaf tissue. In the case of the five-days-old seedling, the visual and radiographic image were overlaid in order to determine the location of the tracer more readily. Imaging of the $\left[{ }^{18} \mathrm{~F}\right] \mathrm{FDG}$ plants was done in an identical manner to the $\left[{ }^{18} \mathrm{~F}\right] \mathrm{FPBA}$ but the $\left[{ }^{11} \mathrm{C}\right]$-photosynthate trials had much shorter phosphor film exposure times because of higher activity levels of the radionuclide.

For the chemical kinetics on maize root development studies, each chemical and concentration, 10-15 kernels of the maize inbred line B73 were rolled into a paper towel. Each paper towel roll was incubated in a different concentration of either PBA (Sigma: P20009-250g), FPBA (Sigma: 417556-25g), BA, or water. Kernels were allowed to germinate in the dark for five 
days at $28{ }^{\circ} \mathrm{C}$. After five days the seedlings were imaged using a Canon EOS Rebel T-6 camera. Primary root length and lateral root density was scored using Image $J^{50}$. For lateral root density, the distance on the primary root from the kernel until the last lateral root was measured and all lateral roots were counted. Afterwards the number of lateral roots was divided by the distance to yield lateral root density per $\mathrm{cm}$. Experiments were repeated at least three times. Averages, standard deviations, and significance (Student's T-Test) were calculated using Microsoft Excel. Analysis of Variance with a post-hoc Tukey Test to correct for multiple testing was done in R. For the connecting letter report the multcompView package in $\mathrm{R}$ was used ${ }^{51}$.

For the PBA/FPBA sensitivity experiment on Zmtls1 and Zmrte seedlings, 3:1 WT:mutant sibling lines of either mutant were used. Per treatment and experiment, 45 kernels were rolled up in paper towel rolls. Kernels in each paper towel roll were germinated in the dark for five days at $28^{\circ} \mathrm{C}$. Pictures of the seedlings were taken with a Canon EOS Rebel T- 6 camera and shoot tissue for each seedling was dissected, DNA isolated and genotyped for Zmtls1 and Zmrte as described $\operatorname{in}^{23-25}$.

Developing siliques of Arabidopsis were treated with either PBA, FPBA, or $\mathrm{H}_{2} \mathrm{O}$ as described in [28]. After ripening of the seeds, all seeds treated with a respective chemical were harvested, sowed on a $\mathrm{H}_{2} \mathrm{O}$ agar plate, stratified for two days at $4{ }^{\circ} \mathrm{C}$, and let to germinate at 28 ${ }^{\circ} \mathrm{C}$ under continuous light. The presence of rootless seedlings was scored.

Roots of seedlings grown in $\mathrm{H}_{2} \mathrm{O}$ or $2 \mathrm{mM}$ FPBA were harvested at five days and the roots were collected and frozen for quantification of B via ICP-MS. The root samples were freezedried for $24 \mathrm{~h}$ in a Thermo Fisher Freezone 1 freeze dryer (Labconco Corp., Kansas City, MO, USA) and then gently ground with mortar and pestle to homogenize. An approximately $0.2 \mathrm{~g}$ representative sample was taken from each sample and microwave-digested (Milestone Ethos 
Plus, SK-12 rotor, Milestone Inc., Shelton, CT, USA) in $2.50 \mathrm{~mL}$ of $14 \mathrm{M} \mathrm{HNO}_{3}$ at $190{ }^{\circ} \mathrm{C}$ for $25 \mathrm{~min}$. Once digested, the samples were diluted to $50 \mathrm{~mL}$ using $18 \mathrm{M} \Omega$-cm water, internal standard was added, and then samples were analyzed via ICP-MS (NexION 300X, KED mode, PerkinElmer Inc., Branford, CT, USA) for endogenous B concentration.

\subsection{Abbreviations}

$\begin{array}{ll}\text { Arabidopsis } & \text { Arabidopsis thaliana } \\ \text { BA } & \text { Boric Acid } \\ \text { FPBA } & \text { Fluoro-Phenylboronic Acid } \\ \text { ICP-MS } & \text { Inductively Coupled Plasma Mass Spectrometry } \\ \text { ICP-OES } & \text { Inductively coupled plasma optical emission spectrometry } \\ \text { PBA } & \text { Phenylboronic acid } \\ \text { TLC } & \text { Thin Layer Chromatography } \\ \text { tls1 } & \text { tassel-less } 1 \\ \text { rte } & \text { rotten ear } \\ \text { Zm } & \text { Zea mays }\end{array}$




\section{References}

1) Warington, $\mathrm{K}$. The Effect of Boric Acid and Borax on the Broad Bean and certain other Plants. Ann Bot 1923, os-37, 629-672.

2) Ahmad, W.; H., M.; S., S.; Niaz, A.; Saifullah Boron Deficiency in Soils and Crops: A Review. In Crop Plant; Goyal, A., Ed.; InTech, 2012 ISBN 978-953-51-0527-5.

3) Pizzorno, L. Nothing Boring About Boron. Integr Med (Encinitas) 2015, 14, 35-48.

4) Shorrocks, V.M. The occurrence and correction of boron deficiency. In Boron in Soils and Plants: Reviews; Dell, B., Brown, P.H., Bell, R.W., Eds.; Springer Netherlands: Dordrecht, 1997; pp. 121-148 ISBN 978-94-010-6352-4.

5) Ishii, T.; Matsunaga, T. Isolation and characterization of a boron-rhamnogalacturonan-II complex from cell walls of sugar beet pulp. Carbohydr. Res. 1996, 284, 1-9.

6) Kobayashi, M.; Matoh, T.; Azuma, Ji. Two Chains of Rhamnogalacturonan II Are CrossLinked by Borate-Diol Ester Bonds in Higher Plant Cell Walls. Plant Physiol. 1996, 110, 1017-1020.

7) O’Neill, M.A.; Warrenfeltz, D.; Kates, K.; Pellerin, P.; Doco, T.; Darvill, A.G.; Albersheim, P. Rhamnogalacturonan-II, a Pectic Polysaccharide in the Walls of Growing Plant Cell, Forms a Dimer That Is Covalently Cross-linked by a Borate Ester: IN VITRO CONDITIONS FOR THE FORMATION AND HYDROLYSIS OF THE DIMER. $J$. Biol. Chem. 1996, 271, 22923-22930.

8) O’Neill, M.A.; Eberhard, S.; Albersheim, P.; Darvill, A.G. Requirement of Borate CrossLinking of Cell Wall Rhamnogalacturonan II for Arabidopsis Growth. Science 2001, 294, 846-849.

9) Goldbach, H.E.; Wimmer, M.A.; Findeklee, P. Discussion Paper: Boron - How can the Critical Level be Defined? J. Plant Nutr Soil Sci 2000, 115-121.

10) Blevins, D.G.; Lukaszewski, K.M. BORON IN PLANT STRUCTURE AND FUNCTION. Annu. Rev. Plant. Physiol. Plant. Mol. Biol. 1998, 49, 481-500.

11) Power, P.P.; Woods, W.G. The chemistry of boron and its speciation in plants. Plant and Soil 1997, 193, 1-13.

12) Dell, B.; Huang, L. Physiological response of plants to low boron. Plant and Soil 1997, 193, 103-120.

13) Steeves, T.A; Sussex, I.M. Patterns in Plant Development; 2nd ed.; Cambridge University Press: Cambridge, 1989;

14) Camacho-Cristóbal, J.J.; Martín-Rejano, E.M.; Herrera-Rodríguez, M.B.; NavarroGochicoa, M.T.; Rexach, J.; González-Fontes, A. Boron deficiency inhibits root cell elongation via an ethylene/auxin/ROS-dependent pathway in Arabidopsis seedlings. $J$. Exp. Bot. 2015, 66, 3831-3840. 
15) Eltinge, E.T. Effect of boron deficiency upon the structure of Zea Mays. Plant Physiol. 1936, 11, 765-778.

16) Lordkaew, S.; Dell, B.; Jamjod, S.; Rerkasem, B. Boron deficiency in maize. Plant Soil 2011, 342, 207-220.

17) Josten, P.; Kutschera, U. The Micronutrient Boron Causes the Development of Adventitious Roots in Sunflower Cuttings. Annals of Botany 1999, 84, 337-342.

18) Landi, M.; Margaritopoulou, T.; Papadakis, I.E.; Araniti, F. Boron toxicity in higher plants: an update. Planta 2019, 250, 1011-1032.

19) Yoshinari, A.; Takano, J. Insights into the Mechanisms Underlying Boron Homeostasis in Plants. Front. Plant Sci. 2017, 8, 1-8.

20) Noguchi, K.; Yasumori, M.; Imai, T.; Naito, S.; Matsunaga, T.; Oda, H.; Hayashi, H.; Chino, M.; Fujiwara, T. borl-1, an Arabidopsis thaliana Mutant That Requires a High Leve1 of Boron'. Plant Phys. 1997, 901-906.

21) Takano, J.; Wada, M.; Ludewig, U.; Schaaf, G.; von Wirén, N.; Fujiwara, T. The Arabidopsis Major Intrinsic Protein NIP5;1 Is Essential for Efficient Boron Uptake and Plant Development under Boron Limitation. Plant Cell 2006, 18, 1498-1509.

22) Fukuda, M.; Wakuta, S.; Kamiyo, J.; Fujiwara, T.; Takano, J. Establishment of genetically encoded biosensors for cytosolic boric acid in plant cells. Plant J. 2018, 763774.

23) Durbak, A.R.; Phillips, K.A.; Pike, S.; O’Neill, M.A.; Mares, J.; Gallavotti, A.; Malcomber, S.T.; Gassmann, W.; McSteen, P. Transport of boron by the tassel-less1 aquaporin is critical for vegetative and reproductive development in maize. Plant Cell 2014, 26, 2978-2995.

24) Chatterjee, M.; Tabi, Z.; Galli, M.; Malcomber, S.; Buck, A.; Muszynski, M.; Gallavotti, A. The boron efflux transporter ROTTEN EAR is required for maize inflorescence development and fertility. Plant Cell 2014, 26, 2962-2977.

25) Chatterjee, M.; Liu, Q.; Menello, C.; Galli, M.; Gallavotti, A. The Combined Action of Duplicated Boron Transporters Is Required for Maize Growth in Boron-Deficient Conditions. Genetics 2017, 206, 2041-2051.

26) Bassil, E.; Hu, H.; Brown, P.H. Use of phenylboronic acids to investigate boron function in plants. Possible role of boron in transvacuolar cytoplasmic strands and cell-to-wall adhesion. Plant Physiol. 2004, 136, 3383-3395.

27) Fang, K.; Gao, S.; Zhang, W.; Xing, Y.; Cao, Q.; Qin, L. Addition of Phenylboronic Acid to Malus domestica Pollen Tubes Alters Calcium Dynamics, Disrupts Actin Filaments and Affects Cell Wall Architecture. PLoS ONE 2016, 11, e0149232.

28) Matthes, M.; Torres-Ruiz, R.A. Boronic acid treatment phenocopies monopteros by affecting PIN1 membrane stability and polar auxin transport in Arabidopsis thaliana embryos. Development 2016, 143, 4053-4062. 
29) Matthes, M.; Torres-Ruiz, R.A. Boronic acids as tools to study (plant) developmental processes? Plant Signal Behav 2017, 12, e1321190.

30) Sarkar, D.; Sheikh, A.A.; Batabyal, K.; Mandal, B. Boron Estimation in Soil, Plant, and Water Samples using Spectrophotometric Methods. Communications in Soil Science and Plant Analysis 2014, 45, 1538-1550.

31) Kaulich, B.; Gianoncelli, A.; Beran, A.; Eichert, D.; Kreft, I.; Pongrac, P.; Regvar, M.; Vogel-Mikuš, K.; Kiskinova, M. Low-energy X-ray fluorescence microscopy opening new opportunities for bio-related research. J R Soc Interface 2009, 6, S641-S647.

32) Wang, X.; Brockman, J.D.; Guthrie, J.M.; Lever, S.Z. Analysis and imaging of boron distribution in maize by quantitative neutron capture radiography. Appl Radiat Isot 2018, $140,252-261$.

33) Thellier, M.; Ripoll, C. Neutron Capture Radiography: Neutron Capture Radiography:a technique for isotopic labelling and analytical imaging with a few stable isotopes. The Scientific World JOURNAL 2006, 6, 671-685.

34) Nudat 2 Available online: https://www.nndc.bnl.gov/nudat2/reCenter.jsp?z=4\&n=4 (accessed on Jul 24, 2019).

35) $\mathrm{Yu}, \mathrm{M}$. Recent developments of the PET imaging agents for metabotropic glutamate receptor subtype 5. Curr Top Med Chem 2007, 7, 1800-1805.

36) Qu, W.; Robert, C.A.M.; Erb, M.; Hibbard, B.E.; Paven, M.; Gleede, T.; Riehl, B.; Kersting, L.; Cankaya, A.S.; Kunert, A.T.; et al. Dynamic Precision Phenotyping Reveals Mechanism of Crop Tolerance to Root Herbivory. Plant Physiology 2016, 172, 776-788.

37) Duran, C.; Arce-Johnson, P.; Aquea, F. Methylboronic acid fertilization alleviates boron deficiency symptoms in Arabidopsis thaliana. Planta 2018, 248, 221-229.

38) Loomis, W.D.; Durst, R.W. Chemistry and biology of boron. Biofactors 1992, 3, 229_ 239.

39) Mach, R.H. Development of 18F- and 11C-Labeled Radiopharmaceuticals. In Continuing Education for Nuclear Pharmacists and Nuclear Medicine Professionals; Jeffrey Norenberg, UNM College of Pharmacy, 2008; Vol. 14, pp. 1-22.

40) Loveland, W.; Morrisey, D.J.; Seaborg, G.T. Modern Nuclear Chemistry; John Wiley \& Sons, Inc., 2006;

41) Jacobson, O.; Kiesewetter, D.O.; Chen, X. Fluorine-18 Radiochemistry, Labeling Strategies and Synthetic Routes. Bioconjug Chem 2015, 26, 1-18.

42) Mossine, A.V.; Brooks, A.F.; Makaravage, K.J.; Miller, J.M.; Ichiishi, N.; Sanford, M.S.; Scott, P.J.H. Synthesis of [18F]Arenes via the Copper-Mediated [18F]Fluorination of Boronic Acids. Org. Lett. 2015, 17, 5780-5783.

43) Bell, R.W.; Rerkasem, B. Boron in Soils and Plants: Proceedings of the International Symposium on Boron in Soils and Plants held at Chiang Mai, Thailand, 7-11 September, 1997; Springer Science \& Business Media, 2012; ISBN 978-94-011-5564-9. 
44) Poza-Viejo, L.; Abreu, I.; González-García, M.P.; Allauca, P.; Bonilla, I.; Bolaños, L.; Reguera, M. Boron deficiency inhibits root growth by controlling meristem activity under cytokinin regulation. Plant Science 2018, 270, 176-189.

45) Nable, Ross O.; Banuelos, Gary S.; Paull, Jeffrey G. Boron Toxicity. In Plant and Soil; Kluwer Academic Publisher: Netherlands, 1997; pp. 181-198.

46) Yan, J.; Springsteen, G.; Deeter, S.; Wang, B. The relationship among pKa, pH, and binding constants in the interactions between boronic acids and diols - it is not as simple as it appears. Tetrahedron 2004, 60, 11205-11209.

47) Aquea, F.; Federici, F.; Moscoso, C.; Vega, A.; Jullian, P.; Haseloff, J.; Arce-Johnson, P. A molecular framework for the inhibition of Arabidopsis root growth in response to boron toxicity. Plant, Cell \& Environment 2012, 35, 719-734.

48) Matthes, M.S.; Robil, J.M.; Tran, T.; Kimble, A.; McSteen, P. Increased transpiration is correlated with reduced boron deficiency symptoms in the maize tassel-less 1 mutant. Physiologia Plantarum 2018, 163, 344-355.

49) Pike, S.; Matthes, M.S.; McSteen, P.; Gassmann, W. Using Xenopus laevis Oocytes to Functionally Characterize Plant Transporters. Current Protocols in Plant Biology 2019, 4, e20087.

50) Schneider, C.A.; Rasband, W.S.; Eliceiri, K.W. NIH Image to ImageJ: 25 years of image analysis. Nature Methods 2012, 9, 671-675.

51) Piepho, H.-P. An Algorithm for a Letter-Based Representation of All-Pairwise Comparisons. Journal of Computational and Graphical Statistics 2004, 13, 456-466. 


\section{Chapter 3: Functional mutants of Azospirillum brasilense elicit beneficial physiological and metabolic responses in Zea mays contributing to increased host iron assimilation while boosting crop yield and seed iron and ferritin content.}

\subsection{Preface}

This project was published on January $6^{\text {th }}, 2021$ in the International Society of Microbial Ecology (ISME) Journal. As first author on the manuscript, I was involved in the administration of the radiotracer (both ${ }^{59} \mathrm{Fe}$ and ${ }^{11} \mathrm{CO}_{2}$ ) to live plants and subsequent autoradiography imaging, $\mathrm{NaI}(\mathrm{Tl})$ detection of radioactivity in the separate plant tissues and for physiology measurements, extraction of radiolabeled metabolites from plant tissues and subsequent metabolite analytical assays, measurement of Fe in the plant tissues via ICP-MS and LA-ICP-MS, PCA analysis, TEM of root samples, in vitro chemotaxis of DIMBOA assays, writing of the first draft of the manuscript, and subsequent edits. All drop plate assays were completed and contributed by Stacy L. Wilder of the University of Missouri Research Reactor Center and the $\mathrm{pH}$ visual assays on seedling roots were contributed by Stephanie Scott of the U. of Missouri Biochemistry Dept. Garren Powell of the Biochemistry Dept. completed and contributed the root gravitropism measurements. Many of the experiments included in this project were collaborative in nature including all of the included authors to varying degrees.

This chapter also includes results from another publication, published on March $14^{\text {th }}$, 2020 in Multidisciplinary Digital Publishing Institute's (MDPI) Agronomy Journal. As second author on this manuscript, I was involved in the method development of the ion chromatography chemiluminescence for iron quantification in seed as well as the subsequent quantification and ferritin measurement in seed. The outdoor plant growth and plant measurements were completed as a group, led by Stephanie Scott of the U. of Missouri Dept. of Biochemistry and chlorophyll 
measurements were contributed by Amber Gearheart, previous member of the U. of Missouri Chemistry Dept.

Housh, A. B.; Powell, G.; Scott, S.; Anstaett, A.; Gerheart, A.; Benoit, M.; Waller, S.; Powell, A.; Guthrie, J. M.; Higgins, B.; Wilder, S. L.; Schueller, M. J.; Ferrieri, R. A. Functional Mutants

of Azospirillum Brasilense Elicit Beneficial Physiological and Metabolic Responses in Zea Mays Contributing to Increased Host Iron Assimilation. The ISME Journal 2021, 1-18.

Scott, S.; Housh, A.; Powell, G.; Anstaett, A.; Gerheart, A.; Benoit, M.; Wilder, S.; Schueller, M.; Ferrieri, R. Crop Yield, Ferritin and Fe(II) Boosted by Azospirillum Brasilense (HM053) in Corn. Agronomy 2020, 10 (3), 394.

\subsection{Introduction}

The Role of Plant Growth Promoting Bacteria in Agriculture: A survey of the literature establishes that many plant growth promoting bacteria (PGPB) can strongly influence plant growth and increase crop yields. Mechanisms have been suggested that support these actions, which include: antagonism toward phytopathogens and induction of plant resistance pathways ${ }^{1}$; phytostimulation through microbial production and secretion of plant relevant hormones like auxin, cytokinins, and gibberellins, as well as nitric oxide ${ }^{2,3}$; improvements in host nitrogen uptake via biological nitrogen fixation $(\mathrm{BNF})^{4}$; and improvements in host micronutrient uptake ${ }^{5,6}$.

Azospirillum brasilense, a Gram-negative bacterium, is perhaps the best studied of the PGPB. It is known to be a prolific grass root colonizer preferring to grow on the outer surface of its host's roots. As a diazotroph this bacteria has the ability to fix $\mathrm{N}_{2}$ and, in some circumstances, transfer that source of nitrogen to its host ${ }^{4}$. A. brasilense can also produce auxin which can affect plant development ${ }^{3}$. Over the years, commercial inoculants of various strains of this bacterium have been developed and tested in the field for their influence on growth performance in a variety of grain crops, including maize, under various environmental conditions ${ }^{7-9}$. In fact, recently we showed that maize inoculated with the functional mutant HM053 A. brasilense 
exhibited $12 \%$ larger stem diameters, $23 \%$ increased leaf thickness and 58\% higher chlorophyll content relative to non-inoculated control plants when surveyed during 2018 and 2019 growing seasons ${ }^{10}$. Furthermore, plant growth promotion resulted in significantly higher crop yields compared to non-inoculated control plants where $34 \%$ and $53 \%$ increases were documented in the number of corn kernels per cob from inoculated plants during the 2018 and 2019 growing seasons, respectively. These phenotypic attributes were ascribed to increased host iron uptake in the presence of the beneficial microbe, which not only improved plant growth, but also had the effect of increasing seed iron content thus improving the nutritional value of the crop product. The Importance of Iron in Plant Growth: Iron is the third most limiting nutrient for healthy plant growth and development; however, this limitation is not attributable to its abundance. Quite the contrary, iron is the fourth most abundant element in the lithosphere. Even so, its bioavailability is limited especially in aerobic, neutral $\mathrm{pH}$ soils because it exists predominantly as insoluble $\mathrm{Fe}^{3+}$ oxyhydroxides that are unusable to plants ${ }^{11,12}$. A deficiency of iron can decrease plant growth affecting crop yield and/or nutritional quality ${ }^{13-15}$, while excess iron can lead to elevated $\mathrm{Fe}^{3+} / \mathrm{Fe}^{2+}$ redox reactions causing cellular damage.

Iron uptake in higher plants follows two mechanisms (Figure 3.2.1.), which are distinct between non-graminaceous plants and graminaceous plants. Non-graminaceous plants utilize a reduction-based strategy (Strategy I) through which protons are exuded from the roots via the actions of membrane-bound $\mathrm{H}^{+}$-ATPases ${ }^{16-29}$. These protons acidify the soil, solubilizing $\mathrm{Fe}^{3+}$. Additionally, membrane-bound $\mathrm{Fe}^{3+}$ reductases ${ }^{20}$ reduce this solubilized iron to $\mathrm{Fe}^{2+}$ enabling its transport across the plasma membrane of the root epidermal cells through the actions of specialized ion transport proteins that are part of the larger ZIP (zinc-regulated transporter and 


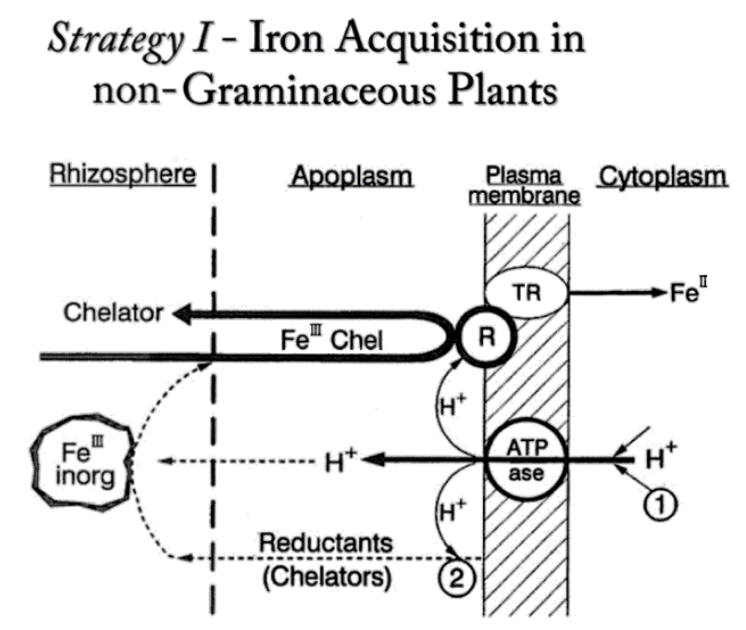

\section{Strategy II - Iron Acquisition in Graminaceous Plants}

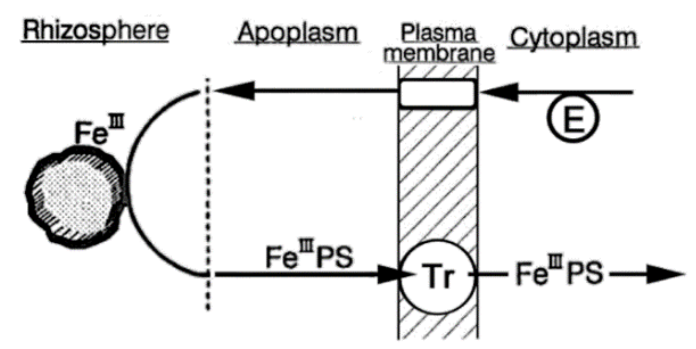

3.2.1. Mechanisms for higher plant assimilation of iron from soil. Non-gramineaceous plants typically follow a Strategy I mechanism in which a proton-ATPase enzyme on the root epidermis secretes protons into the rhizosphere to acidify the soil and solubilize the ferric salts which are typically present as silicates and oxides. Ferric reductase oxidases (R) reduce the ferric to ferrous ions where protein transporters (TR) facilitate uptake into the root cells. Graminaceous plants rely on a Strategy II mechanism whereby they biosynthesize specialized phytosiderophores (PS, or chelating agents) and excrete (E) them into the soil to complex with the ferric iron enabling the roots to take up this chelated form with the aid of specialized transporters (Tr).

iron-regulated transporter) encoding gene family ${ }^{16,17,21-24}$. Graminaceous plants like rice, barley and maize utilize a $\mathrm{Fe}^{3+}$ chelation mechanism (Strategy II) whereby phytosiderophores (PS) are excreted from the roots ${ }^{25}$ where they chelate with $\mathrm{Fe}^{3+}$ in the soil. These PS- $\mathrm{Fe}^{3+}$ chelates are then taken into the root cells through either yellow stripe (YS) or yellow stripe-like (YSL) oligopeptide transporters first characterized in maize ${ }^{26}$. Even with these class distinctions nine ZIP-encoding genes have been identified in the maize genome with little known of their function regarding $\mathrm{Fe}^{2+}$ uptake ${ }^{24}$.

Once assimilated, iron will travel through the apoplastic space to reach the endodermis of the root. The endodermal ring can be a site rich in ion transporters facilitating the trafficking of several metal nutrients to the stele and xylem ${ }^{27}$. It also manifests as a natural barrier to ion trafficking in the form of the Casparian band and suberin lamellae. Here, a layer of waterproof lignin and suberin polymer exists, which can force ion nutrients to pass into the symplast ${ }^{28}$. Once in the vascular core, iron transport in the xylem occurs predominantly as the $\mathrm{Fe}^{3+}-$ citrate 
complex ${ }^{29--32}$. However, iron is also capable of translocation in other forms including nicotianamine chelates (Fe-NA), mugineic acid chelates (Fe-MA) and dihydroxy mugineic acid chelates (Fe-DMA). NA is the direct amino acid precursor to all MAs and is produced from Sadenoysl methionine by nicotianamine synthase ${ }^{33,34}$. Additionally, $\mathrm{Fe}^{2+}$ is capable of xylem translocation in the form of a histidine chelate ${ }^{35}$.

Beneficial Microbes as the Path Forward to Promoting Plant Growth: While evidence is compelling that certain beneficial microbes can influence plant growth through improved micronutrient uptake, we know little about the mechanisms of action, which, if fundamentally understood, could lay the foundation for translating this approach to general farming practices perhaps with greater acceptance by the public.

In the present work, we provide an in-depth analysis into the mechanisms of action for the beneficial effects of $A$. brasilense in maize. Our work relies on $A$. brasilense functional mutants providing a spectrum of biological functions spanning auxin biosynthesis to BNF. The use of functional mutants to examine plant-microbial interactions is not new to the field and has been used extensively in other PGPB systems to examine the effects of associated microorganisms on plant physiology ${ }^{36}$. The functional mutants of $A$. brasilense used in the present study include HM053, a $\mathrm{Nif}^{+}$constitutively expressed strain hyper-fixing $\mathrm{N}_{2}$ and producing high levels of auxin; FP10, a $\mathrm{Nif}^{-}$strain deficient in $\mathrm{N}_{2}$-fixation, but still produces auxin albeit at a slightly lower capacity than HM053; and ipdC, a strain significantly inhibited in its capacity to produce auxin.

\subsection{Results and Discussion}

Bacteria influence host uptake and translocation of iron- Radioactive ${ }^{59} \mathrm{Fe}^{3+}$ and ${ }^{59} \mathrm{Fe}^{2+}$ tracers were applied to roots inoculated with the three strains (HM053, FP10 and ipdC) of A. brasilense. Comparisons in host iron assimilation were made to non-inoculated control plants and to plants 
chemically treated with auxin. As expected from the Strategy II iron uptake mechanism of grasses, non-inoculated maize roots showed higher ${ }^{59} \mathrm{Fe}^{3+}$ uptake than ${ }^{59} \mathrm{Fe}^{2+}$ (Figure 3.3.1. A). Qualitatively, radiographic imaging following tracer administration in non-inoculated control plants revealed high uptake of ${ }^{59} \mathrm{Fe}^{3+}$ in root meristems and lateral roots (Figure 3.3.1. A). ${ }^{59} \mathrm{Fe}^{2+}$ was taken up to a much lesser extent. Quantitative tissue counting and root radiographic images of roots inoculated with the functional mutants of $A$. brasilense revealed very different behavior. The effects of bacteria on host iron assimilation (Figure 3.3.1. A) and root-to-shoot translocation (Figure 3.3.1. B) are striking. Especially with the HM053 functional mutant, such effects on the host plant seem to be tied to biological nitrogen fixing (BNF) and auxin producing capacity of the microorganism. Drop plate assays examining the degree of microbial colonization under our plant growth conditions (Figure 3.3.2) provided evidence that inoculation with all three functional mutants generated between $6.3 \times 10^{6}$ and $3.2 \times 10^{7}$ CFUs $\mathrm{gfw}^{-1}$ of root tissue relative to roots from non-inoculated control plants yielding a substantially lower background microbial count of $6.3 \times 10^{5} \mathrm{CFUs} \mathrm{gfw}{ }^{-1}$. HM053 showed the greatest degree of colonization which could account, in part, for its enhanced performance. However, ipdC and FP10 exhibited the same levels of colonization, yet FP10 outperformed ipdC in influencing host iron assimilation. The extent of colonization does not fully explain the actions here, and thus microbial biological functions must play a role.

The spatial patterning of Fe-59 radioactivity in roots associated with bacteria was different from non-inoculated control roots, with increased ${ }^{59} \mathrm{Fe}^{3+}$ and ${ }^{59} \mathrm{Fe}^{2+}$ accumulation noted at the lateral root junctions (Figure 3.3.3. B-D). FP10 was the exception, which did not exhibit significant ${ }^{59} \mathrm{Fe}^{2+}$ assimilation, but substantial ${ }^{59} \mathrm{Fe}^{3+}$ assimilation (Figure 3.3.3. D). The spatial 


\title{
patterning of radioactivity is consistent with published confocal microscopy images
}

\author{
demonstrating the lateral
}

A.
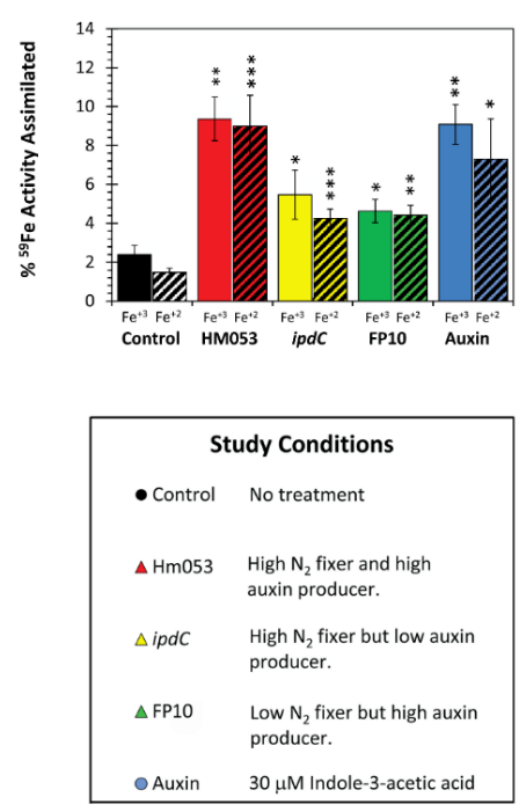

D.

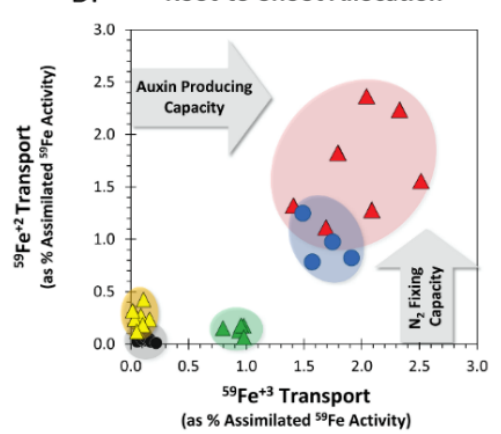

B. Root-to-Shoot Allocation

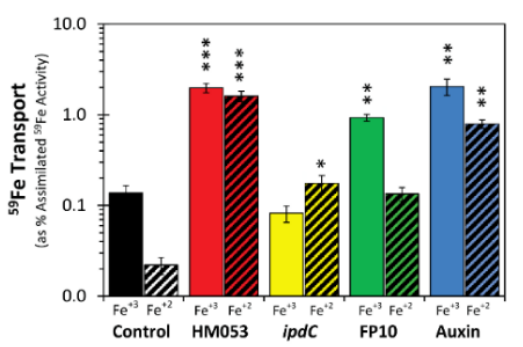

C. Rate of $\left[{ }^{11} \mathrm{C}\right]$ Auxin Biosynthesis

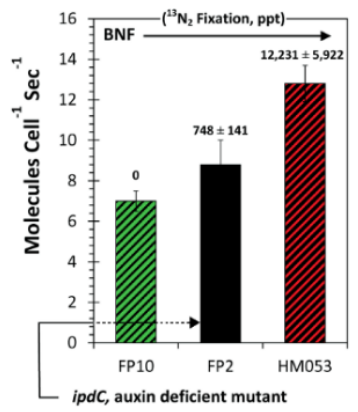

E. Biplot (axes F1 and F2: $100.00 \%$ )

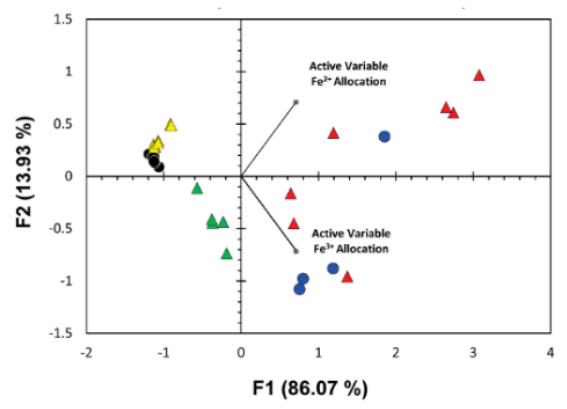

Figure 3.3.1. Radioactive Fe-59 reveals features for $A$. brasilense promotion of host assimilation and whole-plant transport of ferrous iron $\left(\mathrm{Fe}^{2+}\right)$ and ferric iron $\left(\mathrm{Fe}^{3+}\right)$. Panel $\mathrm{A}$ : Bar graph of biological assimilation of radioactive ferrous ${ }^{59} \mathrm{Fe}^{2+}$ and ferric ${ }^{59} \mathrm{Fe}^{3+}$ after $3 \mathrm{hr}$. of incubation of maize roots using $0.74 \mathrm{MBq}$ of tracer. Biological assimilation reflects a combination of root and microorganism assimilation of tracer. Data presented as weight normalized percentage of ${ }^{59} \mathrm{Fe}$ radioactivity administered. Asterisks indicate significant differences of treatment relative to control $\left({ }^{*} \mathrm{P}<0.05 ;{ }^{*} * \mathrm{P}<0.01\right.$; $\left.{ }^{* * *} \mathrm{P}<0.001\right)$. Panel B: Bar graph showing the extent of root-to-shoot transport of radioactive ${ }^{59} \mathrm{Fe}^{2+}$ and ${ }^{59} \mathrm{Fe}^{3+}$ after $3 \mathrm{hr}$. of incubation of maize roots using $0.74 \mathrm{MBq}$ of tracer for the same five study conditions. Data presented as weight normalized percentage of the assimilated ${ }^{59} \mathrm{Fe}$ radioactivity. Asterisks indicate significant differences of treatment relative to control $\left({ }^{*} \mathrm{P}<0.05 ; * * \mathrm{P}<0.01 ; * * * \mathrm{P}<0.001\right)$. Panel C: Bar graph showing auxin biosynthetic rates in A. brasilense using $\left[{ }^{11} \mathrm{C}\right]$ indole radiotracer reveals a trend of increasing rate of production with microbial BNF capacity. Correlations were made to BNF data measured using ${ }^{13} \mathrm{NN}$ (3). Data ( $(\mathrm{SE})$ reflects $\mathrm{N}=4$ biological replicates. Panel D: Correlation plot mapping ${ }^{59} \mathrm{Fe}^{2+}$ allocation against ${ }^{59} \mathrm{Fe}^{3+}$ allocation for the study conditions reveals systematic trends of clustering with treatments that correlate to auxin producing and $\mathrm{N}_{2}$-fixing capacities of the beneficial microbes. Panel E: Principal component analysis correlates ${ }^{59} \mathrm{Fe}$ translocation to biological functions of the beneficial microbes and to chemical treatment using auxin. 
root junctions typically hold high populations of these bacteria ${ }^{3}$. Differences in spatial patterning exhibited by non-inoculated and inoculated roots suggests bacteria assimilation of iron can contribute to a significant portion of the measured root radioactivity detected in the $\mathrm{NaI}$ (PMT) gamma counter even after thorough root washing.

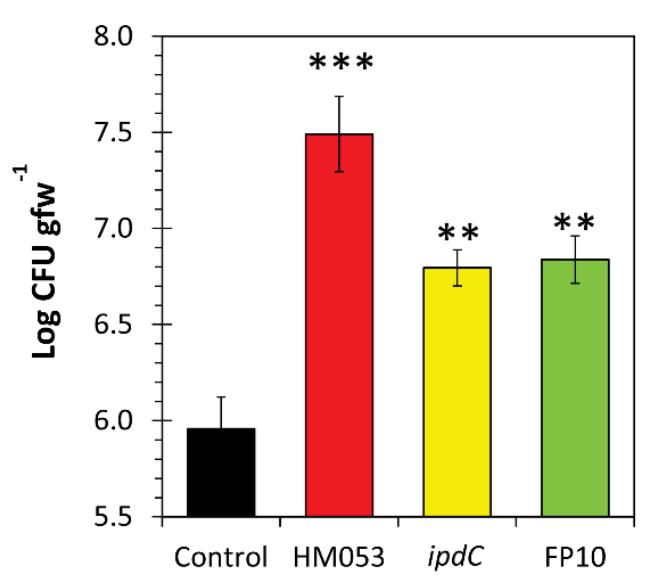

Systematic trends defining in planta translocation (Figure 3.3.3. B\&D) of iron become apparent in the correlation plot (Figure 3.3.1. D) of ${ }^{59} \mathrm{Fe}^{2+}$ transport versus ${ }^{59} \mathrm{Fe}^{3+}$ transport and in the PCA biplot (Figure 3.3.1. E). Here, the information included in our Figure 3.3.2. Drop plate assay analysis on the extent of bacteria colonization of maize roots. Roots were inoculated for $1 \mathrm{hr}$ with ipdC, FP10 and HM053 strains of $A$. brasilense at the 5 day old seedling stage and then transplanted to their respective growth media (aeroponics or solid Turface ${ }^{\mathrm{TM}}$ ). Two separate cohorts of plants were tested with the different strains of bacteria. Data $( \pm \mathrm{SE})$ reflects $\mathrm{N}=4-6$ biological replicates. Asterisks indicate significant differences of treatment compared to controls $(* \mathrm{P}<0.05)$.

allocation measurements were represented by feature vectors (F1 and F2) representing 86.07\% and $13.93 \%$ of the information embedded in the data respectively (for more detail see Supplemental Information). As displayed, each of the treatments clustered together, indicating behavior within a treatment-type that is distinct from other treatments. It shows that ipdC and non-inoculated maize are similar in overall iron allocation behavior, while auxin chemical treatment and HM053 inoculated maize share similar allocation patterns. FP10 is most unique in its allocation patterns. Non-inoculated maize and ipdC inoculated maize clustered away from active variable vectors. This indicates both treatment types do not allocate much iron overall compared to other treatments, and ipdC narrowly allocates more ${ }^{59} \mathrm{Fe}^{2+}$ than ${ }^{59} \mathrm{Fe}^{3+}$. FP10 
inoculated maize are distinctly apart from other treatments in the negative $\mathrm{X}$ - and $\mathrm{Y}$-axis

direction. This is more
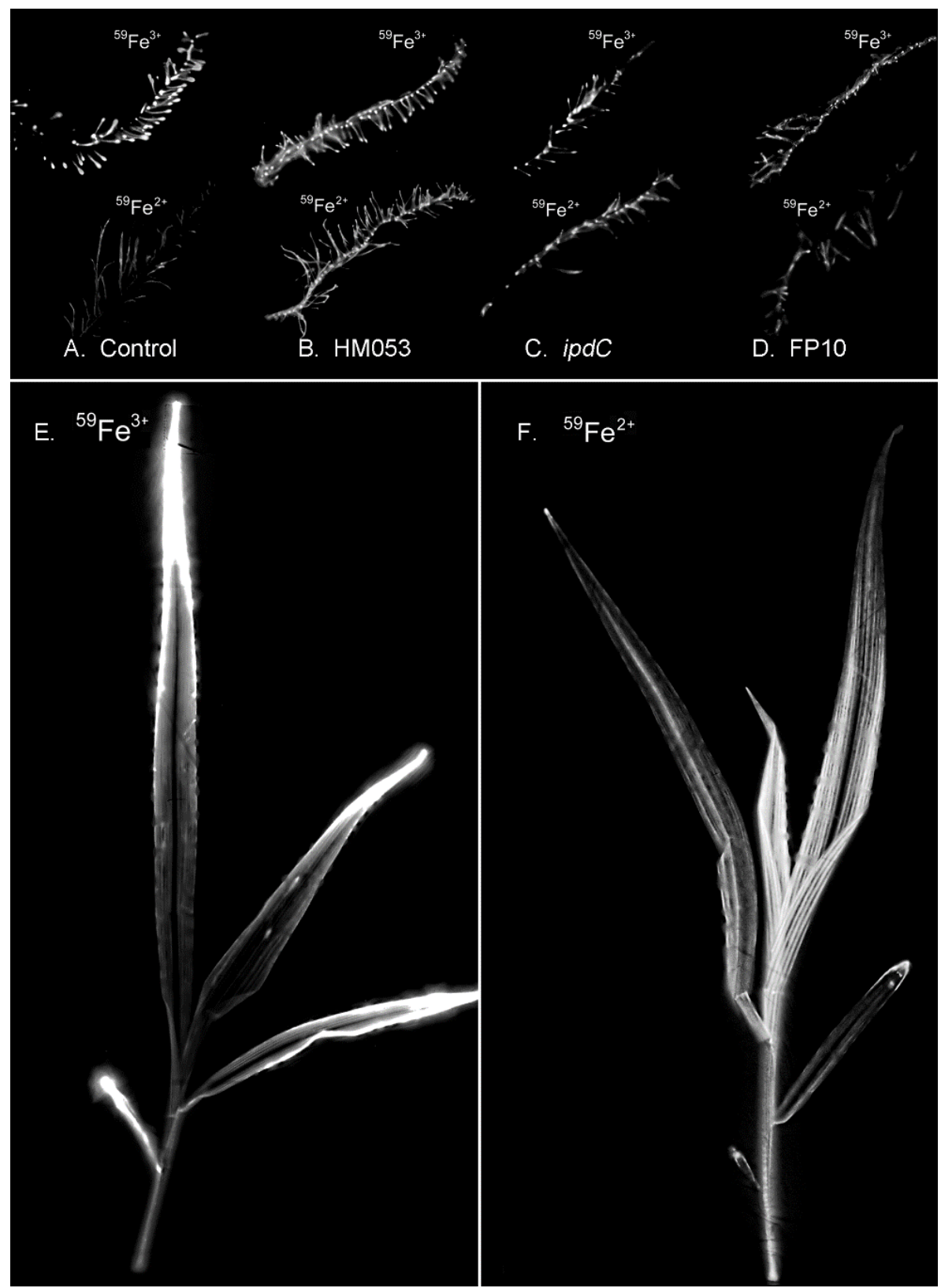

Figure 3.3.3. Radiographic imaging of plant tissues reveal different spatial patterning of radioactive ${ }^{59} \mathrm{Fe}$ as ferric $\left(\mathrm{Fe}^{3+}\right)$ and ferrous $\left(\mathrm{Fe}^{2+}\right)$ ionic forms. Comparisons were made between non-inoculated control roots (Panel A) and roots inoculated with HM053 (Panel B), ipdC (Panel C) and FP10 (Panel D) strains of A. brasilense with ${ }^{59} \mathrm{Fe}^{3+}$ images are shown in the upper portion of each panel while ${ }^{59} \mathrm{Fe}^{2+}$ images are shown in the lower portion. 'Hot' spots of ${ }^{59} \mathrm{Fe}$ radioactivity (both for ${ }^{59} \mathrm{Fe}^{3+}$ and ${ }^{59} \mathrm{Fe}^{2+}$ ) are seen at lateral root junctions. Roots inoculated with the FP10 strain of $A$. brasilense only showed ${ }^{59} \mathrm{Fe}^{3+}$ 'hot' spots. Shoot images for HM053 inoculated plants are shown for ${ }^{59} \mathrm{Fe}^{3+}$ (Panel E) and ${ }^{59} \mathrm{Fe}^{2+}$ (Panel F). Translocation of ${ }^{59} \mathrm{Fe}^{3+}$ to shoots resulted in higher tracer accumulation in all of the leaf tips. Translocation of ${ }^{59} \mathrm{Fe}^{2+}$ to shoots resulted in a higher distribution of tracer in the younger developing leaf tissue. 
positively correlated with $\mathrm{Fe}^{3+}$ allocation and negatively with $\mathrm{Fe}^{2+}$ allocation - which is observed in the correlation plot (Figure 3.3.1. D), as well. Finally, the auxin chemical treatment and HM053 inoculated maize share positions along the active variable vectors, indicating they have the largest allocation percentages across all treatment types for both ${ }^{59} \mathrm{Fe}^{3+}$ and ${ }^{59} \mathrm{Fe}^{2+}$. HM053 inoculated maize tend to have the largest allocation values, especially for the ${ }^{59} \mathrm{Fe}^{2+}$ as noted by the majority of points along that active variable vector while the auxin chemical treatment tends to show more ${ }^{59} \mathrm{Fe}^{3+}$ than ${ }^{59} \mathrm{Fe}^{2+}$ allocation as indicated by its position on the biplot.

To summarize, allocation of ${ }^{59} \mathrm{Fe}^{2+}$ appeared stimulated by BNF while both ${ }^{59} \mathrm{Fe}^{3+}$ and ${ }^{59} \mathrm{Fe}^{2+}$ appeared stimulated by the auxin biosynthetic capacity of the inoculated bacterium. This assumes bacteria-borne auxin or indole-like substrates move from the microorganism into their host. Exogenously applied chemical auxin stimulated host iron assimilation and whole-plant iron translocation just as HM053 inoculated plants did (Figure 3.3.1. A\&B). BNF and auxin biosynthesis are not mutually exclusive microbial functions. BNF capacity seems to impact bacterial ability to produce auxin (Figure 3.3.1. C), although it is not essential for that function. FP10, the BNF deficient strain, was able to biosynthesize auxin at a rate of $7.0 \pm 0.4$ molecules cell $^{-1} \mathrm{~s}^{-1}$, slightly less than the $9.2 \pm 0.9$ molecules cell ${ }^{-1} \mathrm{~s}^{-1}$ production rate demonstrated by the FP2 wild-type strain and significantly less than the $13.4 \pm 0.9$ molecules cell ${ }^{-1} \mathrm{~s}^{-1}$ auxin production rate of the HM053 strain. Though not directly measured by our group using tracer analysis as done with other mutants, ipdC's capacity to produce auxin was reported to be approximately $10 \%$ that of the wild-type strain ${ }^{41}$.

Bacteria influence host cellular-scale iron distribution- Laser ablation-inductively coupled-mass spectrometry (LA-ICP-MS) was applied to root sections to corroborate radiotracer data by spatially mapping stable Fe-56 at cellular scales. Significantly elevated Fe-56 accumulation was 
observed in the endodermal ring from HM053, but not ipdC or FP10 inoculated plants, nor in non-inoculated control root sections (Figure 3.3.4. A\&B). Digestive ICP-MS analysis enabling measurement of absolute $\mathrm{Fe}-56$ concentrations in roots and leaves (Figure 3.3.4. C) showed that HM053 significantly increased whole-plant tissue iron levels 4-fold in leaves and 10-fold in roots relative to controls, corroborating radiotracer experiments. Plants inoculated with ipdC showed lower levels of Fe-56 than non-inoculated controls. Though not entirely consistent with our radiotracer data, we note that levels of Fe-59 allocation over the short term were very small such that over a longer period of plant growth, iron accumulation could manifest in the same or lower iron signature than controls. Similarly, FP10 showed only slightly lower or same levels of iron as controls.

To investigate high iron accumulation in the endodermal ring of HM053 inoculated plants, we used transmission electron microscopy to examine the radial walls of root endodermal cells (Figure 3.3.4. D). Morphological differences were seen between non-inoculated and HM053inoculated roots with HM053 showing more prominent development of the Casparian band between cell walls. The Casparian band is a layer of waterproof lignin and suberin located at the endodermal ring. This barrier forces iron nutrients to pass from the apoplast into the symplast as they move to the stele and vascular core ${ }^{27}$. Once iron has entered the symplast, it is bound to various chelators that facilitate its transport as $\mathrm{Fe}^{3+}$-chelate. However, the endodermal ring can be a site harboring a rich density of metal transporters that may transport $\mathrm{Fe}^{2+}$ as well as other metals ${ }^{27}$.

High accumulation of iron in the endodermal ring seen with HM053 inoculation implies the bacteria must influence cellular transport of this element, whether as $\mathrm{Fe}^{2+}$ or $\mathrm{Fe}^{3+}$, from the outer epidermal cells to the endodermal ring. Because our LA-ICP-MS analyses were conducted on freeze dried root sections, likely metal ion distributions across each cell will be affected. 

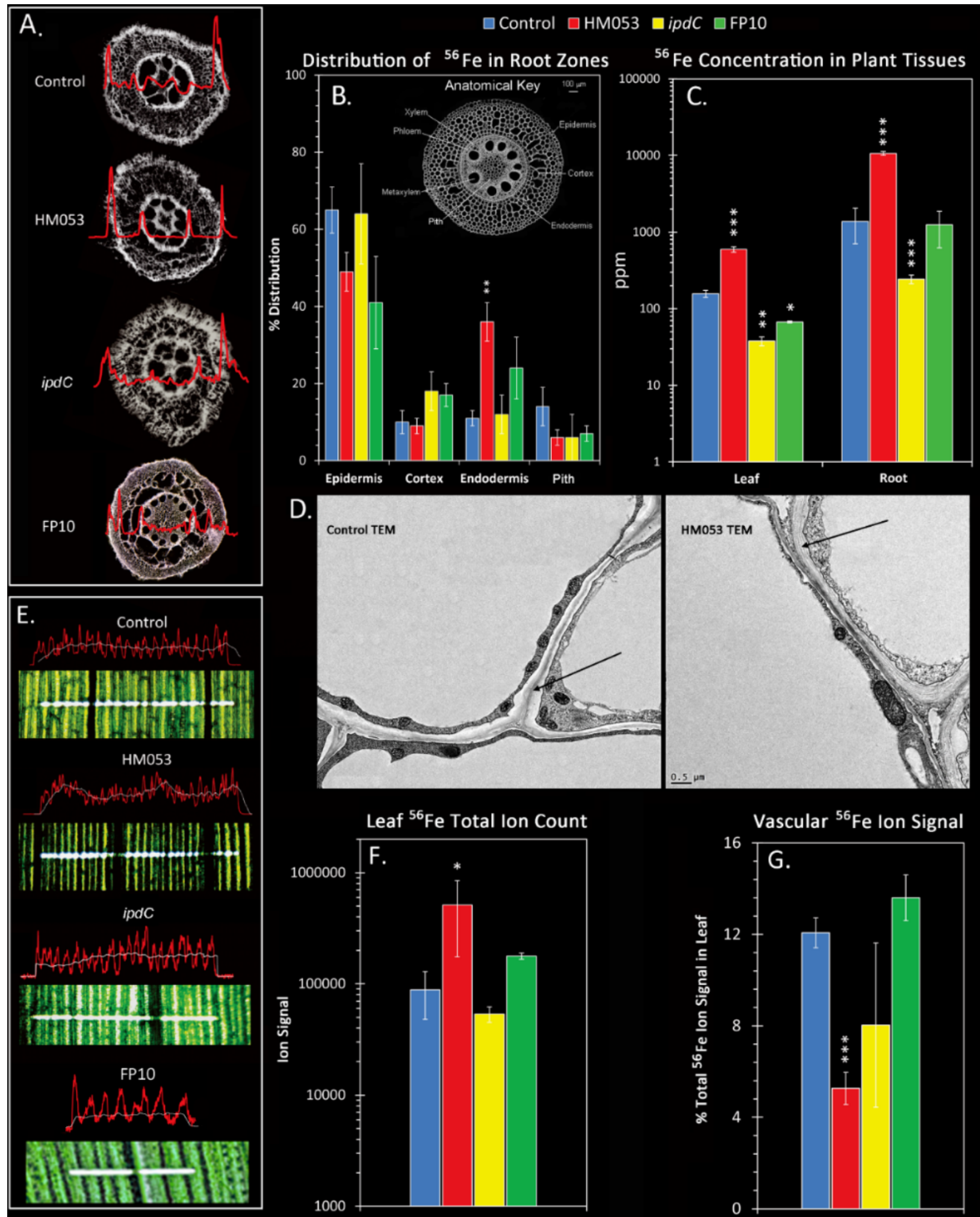
Figure 3.3.4. LA-ICP-MS and TEM reveal spatial patterning of Fe-56 in plant tissues that correlates with root cellular morphological changes due to beneficial microbes. Panel A: Representative Fe-56 ion signals (in red) from the laser ablation of $100 \mu \mathrm{m}$ root sections (taken $1 \mathrm{~cm}$ from the root tip) from non-inoculated control plants, and plants inoculated using HM053, ipdC and FP10 bacteria. Panel B: Data from ablation tracks was averaged across N=6 biological replicates, and presented graphically as \% distribution of the integrated Fe-56 ion signal for the different regions of the root cross section (epidermis, cortex, endodermis and inner pith region). These regions are identified on the inset anatomical key. HM053 treatment resulted in significantly higher iron levels in the endodermis than controls. Panel C: Absolute concentrations of Fe-56 (ppm) in leaves and roots were determined from direct injection ICP-MS of digested tissues and compared against NIST standards. Panel D: Transmission electron microscopy images on endodermal root cells reveal morphological differences between cell walls of non-inoculated control roots and those from HM053. HM053 inoculated roots appeared to have a more defined Casparian band in endodermal radial root walls than in control plants (highlighted by black arrows). Panel E: Representative Fe-56 signals from $40 \mu \mathrm{m}$ diameter x $2 \mathrm{~mm}$ laser tracks rastered across freeze dried leaf tissues harvested from non-inoculated, HM053, ipdC and FP10 inoculated plants. The periodic patterning of the iron ion signals (red lines) presumably are due to the higher disposition of iron in leaf cell walls after the freeze drying process. The white lines reflect an average ion signal across the laser track. HM053 elicits a unique patterning with lower iron signal in and around vascular tissues that was not seen with the other beneficial microbes. Panel F: The ion signal for Fe-56 was integrated across the laser ablation track and plotted as a function of biologic type. HM053 showed a higher integrated iron signal than non-inoculated control, as well as ipdC and FP10 inoculated plants. Panel G: Relative percent of the Fe-56 ion signal in vascular tissue was plotted as a function of biologic type. HM053 inoculation decreased the relative percentage of iron in leaf vascular tissue suggesting greater mobility. Data $( \pm \mathrm{SE})$ reflected $\mathrm{N}=4-6$ biological replicates. Asterisks indicate significant differences of treatment relative to control $(* \mathrm{P}<0.05 ; * * \mathrm{P}<0.01 ; * * * \mathrm{P}<0.001)$

Therefore, we are reluctant to draw any conclusions at this time on whether apoplastic transport dominated iron cellular trafficking. In future studies, we hope to re-examine this behavior once a cryostage is constructed enabling laser ablation of fresh tissue sections.

There is evidence, however, that cellular iron transport is promoted by HM053 from leaf tissue analyses using LA-ICP-MS (Figure 3.3.4. E-G). Here, HM053 inoculated plants significantly increased Fe-56 transport out of foliar vascular tissues (Figure 3.3.4. G) relative to controls while ipdC and FP10 inoculated plants showed no difference in vascular tissue iron levels compared to controls.

Bacteria influence host use of its carbon resources- The effect of HM053, ipdC and FP10 inoculants on their host's physiological and metabolic posture was examined to better understand the mechanism for bacterial induced enrichment of plant iron. Administration of radioactive ${ }^{11} \mathrm{C}$ to live plants as $\left[{ }^{11} \mathrm{C}\right] \mathrm{CO}_{2}$ allowed the measurement of leaf carbon fixation, export of ${ }^{11} \mathrm{C}$ photosynthates from shoots-to-roots, and exudation of ${ }^{11} \mathrm{C}$-substrates to the surrounding rhizosphere (Figure 3.3.5. A-C). These experiments showed that the functional mutants induced significantly different host physiological responses relative to non-inoculated control plants and 

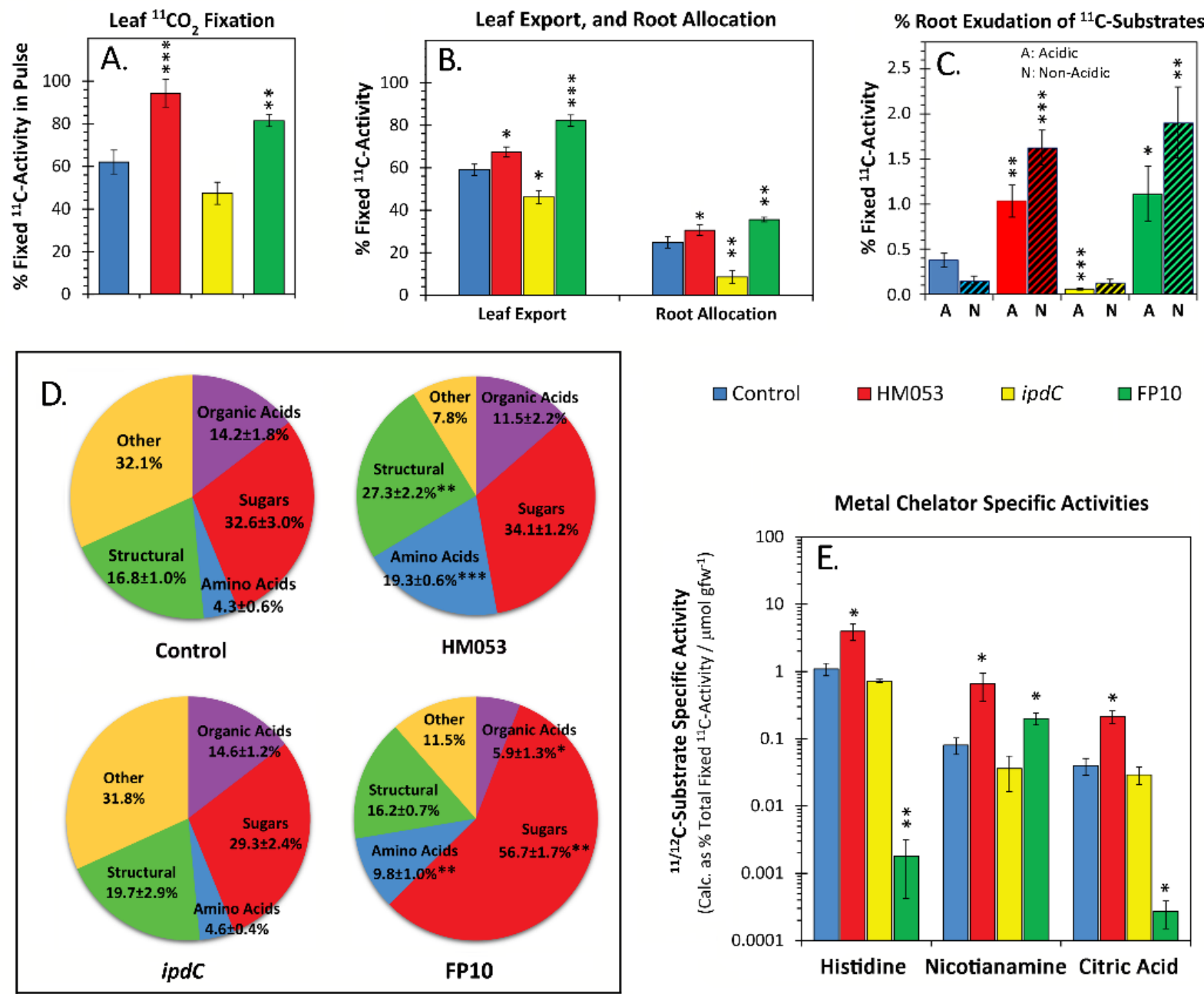

$$
\square \text { Control } \quad \square \mathrm{HM} 053 \quad \square i p d C \quad \square \mathrm{FP} 10
$$

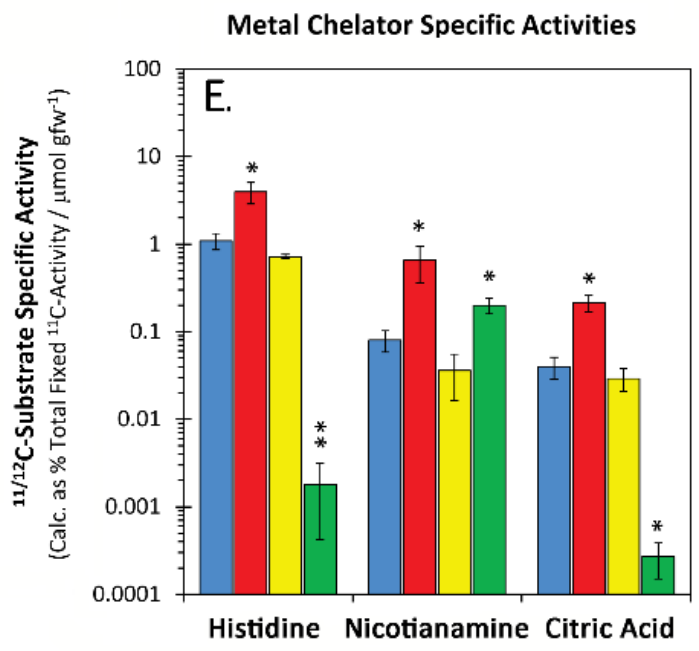

Figure 3.3.5. Carbon-11 aids in mapping maize physiological and metabolic responses to bacteria inoculation. Panel A: Leaf tissue normalized ${ }^{11} \mathrm{CO}_{2}$ fixation presented as $\%{ }^{11} \mathrm{C}$-activity in the pulse applied to the leaf cuvette. Panel B: Leaf export and root allocation of ${ }^{11} \mathrm{C}$-photosynthates (measured at $3 \mathrm{hr}$. post ${ }^{11} \mathrm{CO}_{2}$ pulse) presented as $\%$ fixed ${ }^{11} \mathrm{C}$-activity by the plant. Panel $\mathrm{C}$ : Root exudation of ${ }^{11} \mathrm{C}$-substrates $(\mathrm{A}=$ acidic substrates; $\mathrm{N}=$ non-acidic substrates measured at $3 \mathrm{hr}$. post ${ }^{11} \mathrm{CO}_{2}$ pulse) presented as \% fixed ${ }^{11} \mathrm{C}$-activity by the plant. Panel D: Metabolic landscape reflecting the partitioning of 'new' carbon $\left({ }^{2}{ }^{11} \mathrm{C}\right)$ into different metabolic pools of the load leaf tissue. Panel E: $\left[{ }^{11 / 12} \mathrm{C}\right]$-histidine, $\left[{ }^{11 / 12} \mathrm{C}\right]$-nicotianamine and $\left[{ }^{11 / 12} \mathrm{C}\right]$-citric acid specific activities (SA) levels measured in leaf tissue $20 \mathrm{~min}$. after tracer administration and represented as $\%$ total ${ }^{11} \mathrm{C}$-activity $/ \mu \mathrm{mol}$ substrate $\mathrm{gfw}^{-1}$ of tissue. Data $( \pm \mathrm{SE})$ reflects $\mathrm{N}=7-9$ biological replicates. Asterisks indicate significant differences relative to control $(* \mathrm{P}<0.05$; $* * \mathrm{P}<0.01 ; * * * \mathrm{P}<0.001)$.

each other. Increased carbon input via $\left[{ }^{11} \mathrm{C}\right] \mathrm{CO}_{2}$ fixation with $\mathrm{HM} 053$ and FP10 inoculants correlated with higher root allocation of carbon resources and root exudation (Figure 3.3.5. AC). Exudation of acidic substrates with these functional mutants was 2.8-fold and 2.1-fold higher for HM053 and FP10 inoculated plants, respectively, than non-inoculated control plants (Figure 3.3.5. C). This behavior was confirmed with a $\mathrm{pH}$ visual assay (Figure 3.3.6.). Taken together, 
these observations could help explain the noted increase in Strategy II iron assimilation using HM053 and FP10 inoculants because certain acidic exudates behave as chelating agents of $\mathrm{Fe}^{3+}$. Additionally, differe nt patterns of metabolic partitioning of 'new' carbon (as $\left.{ }^{11} \mathrm{C}\right)$ into the plant's metabolic landscape were noted between the functional mutants and non-inoculated control plants (Figure 3.3.5. D). In particular, HM053 and FP10 inoculation significantly increased ${ }^{11} \mathrm{C}$ partitioning into the amino acid (AA) pool 4.5-fold and 2.3-fold, respectively, relative to controls. Inoculation with ipdC did not have an effect on the AA pool relative to controls.

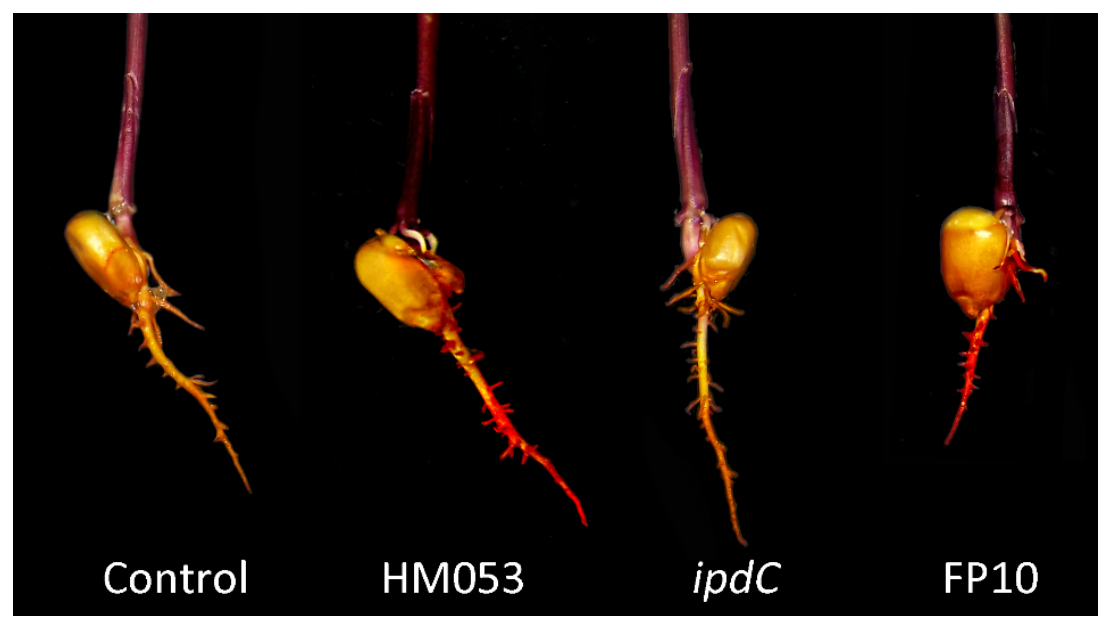

Other subtle differences in the metabolic landscape were seen including HM053 influence increasing host 'new' carbon partitioning into structural metabolite pools

Figure 3.3.6. Visual assay of root $\mathrm{pH}$ in 3 day old maize seedlings reveals a higher level of acidic $\mathrm{pH}$ surrounding HM053 and FP10 inoculated roots. Visualization was acquired by adding bromothymol blue $\mathrm{pH}$ indicating dye to Gelrite ${ }^{\mathrm{TM}}$ semi-solid growth media. Areas on the root showing a red coloration are indicative of acidic $\mathrm{pH}$. Seedlings were extracted at 3 days, washed and digital photos taken.

(Figure 3.3.5. D). This

strongly correlates with our prior work demonstrating that plants inoculated with HM053 had thicker leaves and larger stems than non-inoculated controls ${ }^{10}$.

Insight into particular points of metabolic regulation and the bearing these actions may have on host iron transport prompted examination of individual metabolite ${ }^{11 / 12} \mathrm{C}$ specific activities (SA). Large changes in metabolite SA values reflect high metabolic input of 'new' carbon resources while substrate endogenous concentrations are being depleted by the host's demands 
for a particular metabolite. SA values for $\left[{ }^{11 / 12} \mathrm{C}\right]$ histidine, $\left[{ }^{11 / 12} \mathrm{C}\right]$ nicotianamine, and $\left[{ }^{11 / 12} \mathrm{C}\right]$ citric acid were significantly elevated with HM053 inoculation relative to control plants. Plants inoculated with FP10 showed significant elevation in $\left[{ }^{11 / 12} \mathrm{C}\right]$ nicotianamine SA, but significantly lower values for $\left[{ }^{11 / 12} \mathrm{C}\right]$ histidine and $\left[{ }^{11 / 12} \mathrm{C}\right]$ citric acid relative to controls. ipdC inoculation had no effect on SA values (Figure 3.3.5. E).

Histidine, one of the least abundant AAs in plant metabolism, plays important roles as a proton shuttle substrate in the electron transport train of the plant's photosynthetic PSII system ${ }^{68}$. HM053 promoted increased $\left[{ }^{11} \mathrm{C}\right] \mathrm{CO}_{2}$ fixation (Figure 3.3.5. A) suggesting up-regulation of leaf photosynthesis. However, this may also be due in part to observed increases in leaf chlorophyll with HM053 inoculation ${ }^{10}$.

We note that histidine is also an important chelating agent in the cell-to-cell transport of divalent metals including $\mathrm{Fe}^{2+} 50$. Hence, the significant increase in $\left[{ }^{11 / 12} \mathrm{C}\right]$ histidine SA with HM053 inoculation may, in part, contribute to the increased translocation of ${ }^{59} \mathrm{Fe}^{2+}$ (Figure 3.3.1. B). Inoculations with $i p d C$ or FP10 bacteria had no effect or decreased $\left[{ }^{11 / 12} \mathrm{C}\right]$ histidine SAs relative to controls, respectively. Recall that ipdC showed slightly elevated levels of ${ }^{59} \mathrm{Fe}^{2+}$ allocation relative to controls while FP10 showed a significant reduction in ${ }^{59} \mathrm{Fe}^{2+}$ allocation.

Like histidine, nicotianamine and citric acid are also important chelating agents of iron, facilitating transport through the plant's vascular architecture ${ }^{51}$. Both HM053 and FP10 inoculates significantly elevated $\left[{ }^{11 / 12} \mathrm{C}\right]$ nicotianamine SAs while only HM053 increased $\left[{ }^{11 / 12} \mathrm{C}\right]$ citric acid SA. Together, these changes in host metabolic regulation are relevant to explaining why HM053 was the better performer in stimulating host iron assimilation and allocation. 
Bacteria-borne indole could influence host root function- Additional influences of $A$. brasilense on host root performance were examined to better understand the mechanisms for iron enrichment. Ethylene, auxin, DIMBOA (2,4-dihydroxy-7-methoxy-1,4-benzoxazin-3-one) , and indole are interrelated in regulation and/or root metabolic and physiological functions. DIMBOA, a benzoxazinoid secondary metabolite in maize, shares a common branch point with auxin biosynthesis from the shikimate pathway at the indole precursor (Figure 3.3.7.). Therefore, the bacteria's own auxin producing capabilities might influence host root auxin biosynthesis and/or sensing.

In all higher plants, indole is produced from indole-3-glycerol phosphate and channeled into different pathways. Indole is transformed into tryptophan, and later auxin, by the tryptophan synthase- $\alpha$ subunit and tryptophan synthase- $\beta$ subunit ${ }^{52,53}$. In maize specifically, indole is also produced by the BX1 enzyme as an intermediate in the production of DIMBOA, and other benzoxazinoid secondary metabolites, which play important roles as allelochemicals in maize defense $\mathrm{e}^{54,55}$. However, indole and its role in maize metabolism is more complicated than serving as a metabolic intermediate for DIMBOA or auxin biosynthesis. Aside from the previously mentioned routes, it can also be formed by indole-3-glycerol phosphate lyase (IGL), which subsequently releases it as a volatile in response to herbivore attack ${ }^{56}$. Indole release has been shown to occur earlier than other induced biochemical responses where the $I g l$ gene is induced in response to the actions of certain fatty acid derivatives such as $N$-(17-hydroxylinolenoyl)-Lglutamine isolated from regurgitate of some herbivore-pests ${ }^{57}$. Hence, as a chemical agent, indole can play an important role in maize defense priming, preparing systemic tissues and/or neighboring plants for an impending attack ${ }^{58}$. 
Ancillary data was acquired supporting the premise that bacteria-borne indole may be
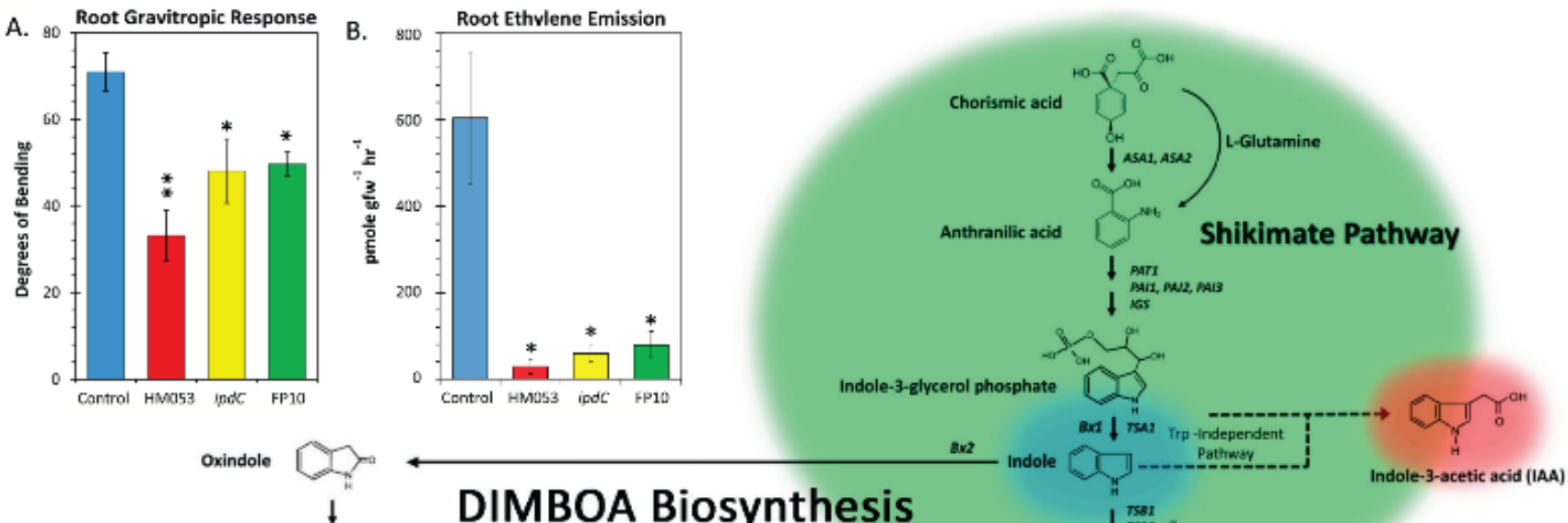

DIMBOA Biosynthesis

Oxindole

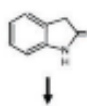

tous

$\downarrow$

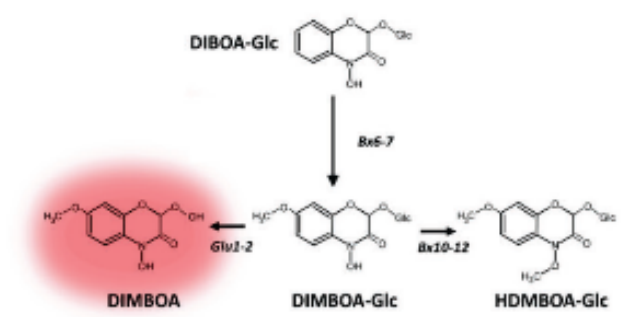

c.

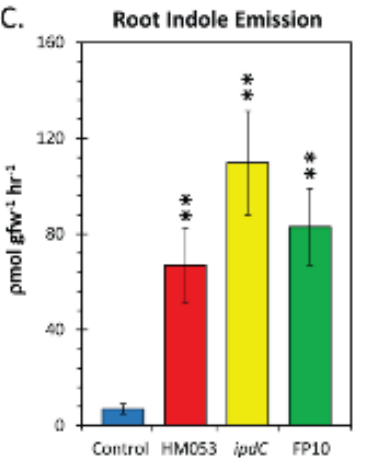

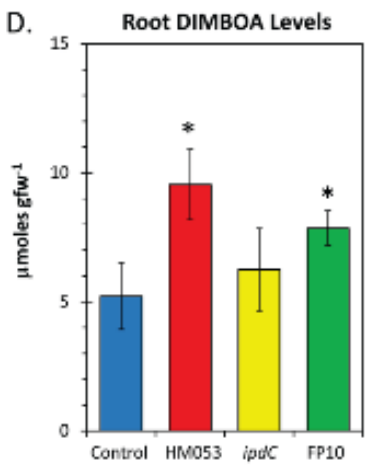

Plastid

Cytosol

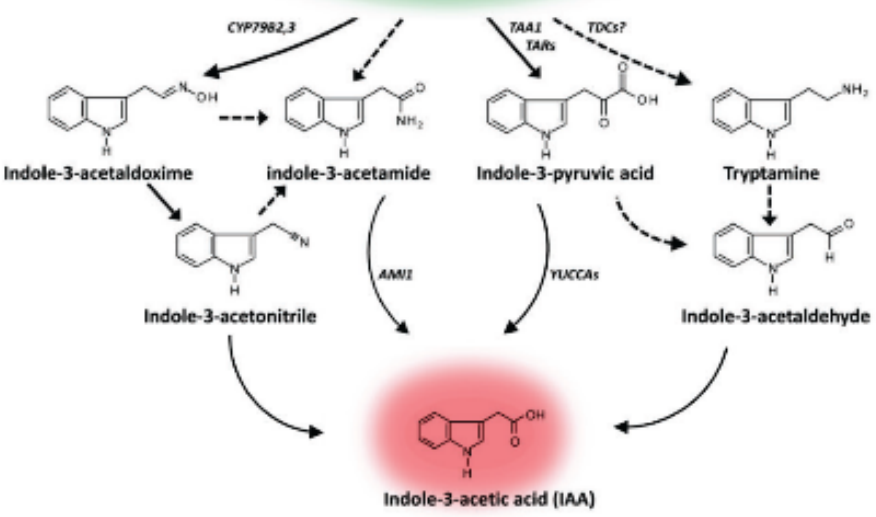

Auxin Biosynthesis

Figure 3.3.7. Shared metabolic branch point in the biosynthesis of Auxin and DIMBOA. The biosynthesis of auxin precursors such as indole-3-glycerol phosphate, indole and L-Trp takes place in plastids and are generated via the shikimate pathway. Four putative L-Trp pathways for auxin biosynthesis are shown. Enzymes known to operate these pathways are shown in italics. Solid pathway arrows reflect active processes. Dashed arrows are suggested to exist, but have not been proven. Benzoxazinoid biosynthesis yielding DIMBOA and its conjugate analogs share a common metabolic branch point with auxin as indole. Panel A: Gravitropic responses of root tips are associated with auxin biosynthesis and/or sensing. Inoculation of roots using HM053, ipdC and FP10 bacteria reduced root gravitropic responses relative to controls. Panel B: Auxin and ethylene biosynthesis are synergistic - inoculation of roots using HM053, ipdC and FP10 bacteria reduced root ethylene emission rates relative to noninoculated controls. Panel C: Root indole emission rates were significantly elevated relative to controls in roots inoculated with HM053, ipdC and FP10 bacteria. Panel D: Root DIMBOA concentrations were significantly increased relative to control in roots inoculated with HM053 and FP10 bacteria and unchanged for ipdC bacteria. Data $( \pm \mathrm{SE})$ reflects $\mathrm{N}=6-8$ biological replicates. Asterisks indicate significant differences relative to control $\left({ }^{*} \mathrm{P}<0.05 ; * * \mathrm{P}<0.01 ; * * * \mathrm{P}<0.001\right)$. 
influencing host metabolism and root physiology. First, root behavior related to auxin (root gravitropism) was investigated due to the genotypic differences in the bacterial mutant strains' ability to produce auxin. It was noted that FP10, ipdC and HM053 strains all exerted similar reductions in root gravitropism (Figure 3.3.7. A), a phenotype highly dependent on auxin biosynthesis and/or sensing in the root tip ${ }^{59}$. Because this was elicited by all bacteria strains, it is suspected that infusion of bacteria-borne indole, rather than auxin, may offset auxin homeostasis at the root tip. All functional mutants used in the present study should produce indole, while solely ipdC is deficient in the major pathway of auxin formation from indole.

Auxin and ethylene biosynthesis are mutually intertwined ${ }^{60}$ with auxin exerting control over 1-aminocyclopropane-1-carboxylate oxidase (ACC oxidase), the controlling enzyme in ethylene biosynthesis. ACC oxidase is a member of the non-heme $\mathrm{Fe}^{2+}$ dependent family of oxygenases and oxidases in higher plants ${ }^{61}$. Hence, there could be a strong connection between plant auxin regulation and iron assimilation through the auxin-ethylene relationship. Indeed, it was noted that treatment of plants with exogenous auxin increased uptake of $\mathrm{Fe}^{3+}$ and $\mathrm{Fe}^{2+}($ Figure 3.3.1 A\&B). In root volatile emissions measurements all functional mutants caused significant reductions in host root ethylene emissions relative to non-inoculated controls (Figure 3.3.7. B). This behavior is similar to previously reported results using the endophyte Herbaspirillum seropedicae (strain SmR1) in rice ${ }^{62}$. Past published work suggests that PGPB harboring ACC deaminase, the bacteria enzyme that breaks down ACC, lower host ethylene emissions ${ }^{36,63}$, yet physiology studies consistently predict that lower ethylene levels increase plant sensitivity to environmental stress $^{64,65}$. While there may be some truth to both sides of this paradox, we believe the story is more complex than a simple relationship between microbial ACC deaminase, plant ethylene regulation and plant growth. For instance, it has been previously documented that some members 
of the PGPB family Azospirillum sp. do not possess the ACC deaminase gene ${ }^{66,67}$. This is true of A. brasilense, yet our work demonstrates that root ethylene emissions are significantly reduced with these $A$. brasilense mutants. Furthermore, we contend that the relationship between microbial ACC deaminase and plant growth promotion may not be generalizable across all PGPB interactions since HM053 A. brasilense was noted to promote plant growth and, in fact, increase crop yield in maize ${ }^{10}$. Instead, we suggest host ethylene regulation could be coupled to microbial indole synthesis, the precursor for auxin. In the present work, measurements of root indole levels showed that all three functional mutants of $A$. brasilense significantly elevated indole emission rates relative to non-inoculated control plants (Figure 3.3.7. C). We suspect this effect could be attributed to the trafficking of bacteria-borne indole into the host root noting that while $i p d C$ is deficient in producing auxin, its upstream metabolic machinery for producing indole should be intact. In fact, ipdC elicited a higher elevation in root indole emission than HM053 or FP10 inoculants.

There are numerous examples in the literature attesting to the complex interaction between ethylene and auxin ${ }^{68-71}$. Studies have shown that ethylene stimulates auxin biosynthesis and upregulates the transcription of several auxin transporters including, PIN1, PIN2, AUX1 ${ }^{72-74}$. Ethylene-induced auxin production is localized in the root tip ${ }^{74}$ and the auxin signal is subsequently redistributed upward through polar auxin transport. We propose that microbial indole infusion in the upper proximal zones of the roots, where the bacteria tend to colonize ${ }^{3}$, could locally boost auxin biosynthesis in these tissues thereby impairing auxin biosynthesis in root tip by disrupting its natural redistribution gradient, and thereby causing a reduction in ethylene emission. This theory is supported by the fact that all three functional mutants caused a 
significant decrease in root gravitropic response - an action that is linked to root tip auxin regulation.

Finally, HM053 and FP10 inoculants significantly elevated levels of root DIMBOA (Figure 3.3.7. D) while ipdC did not. The data presented in this figure represents the sum of the endogenous root pool and surface-bound exudate. The influence of HM053 and FP10 on root DIMBOA may, in part be due to the increased indole pool caused by the bacteria, but then the lack of a response here from ipdC cannot be explained. A theory here is these functional mutants may influence the partitioning of host carbon resources between DIMBOA and auxin biosynthesis, but that remains to be tested in future radiotracer studies. We note that while DIMBOA is typically considered part of maize's natural defense mechanism, it can also play a role as a $\mathrm{Fe}^{3+}$ chelating agent for promoting plant uptake of iron $^{56}$. The observed increase in root DIMBOA levels with HM053 and FP10 inoculation may, in part, contribute to observed elevations in ${ }^{59} \mathrm{Fe}^{3+}$ assimilation with these inoculants.

One question that remains is why A. brasilense would promote production of a bactericide that could potentially be harmful to the microorganism's health. We note that past work has shown that Pseudomonas putida, another PGPB, was tolerant of DIMBOA ${ }^{75}$. To test the theory that certain functional mutants of $A$. brasilense were also tolerant of DIMBOA, we conducted a chemotaxis bioassay examining microbial growth at $0 \mathrm{mM}, 0.02 \mathrm{mM}, 0.1 \mathrm{mM}$ and $0.5 \mathrm{mM}$ doses of DIMBOA added to the growth media. These doses were selected based on earlier work reported with P. putida ${ }^{75}$. In parallel with these assays we conducted a DIMBOA stability test over a $27 \mathrm{hr}$ window (Figure 3.3.8.). Spanning 27 hours, DIMBOA underwent only 15\% degradation. 

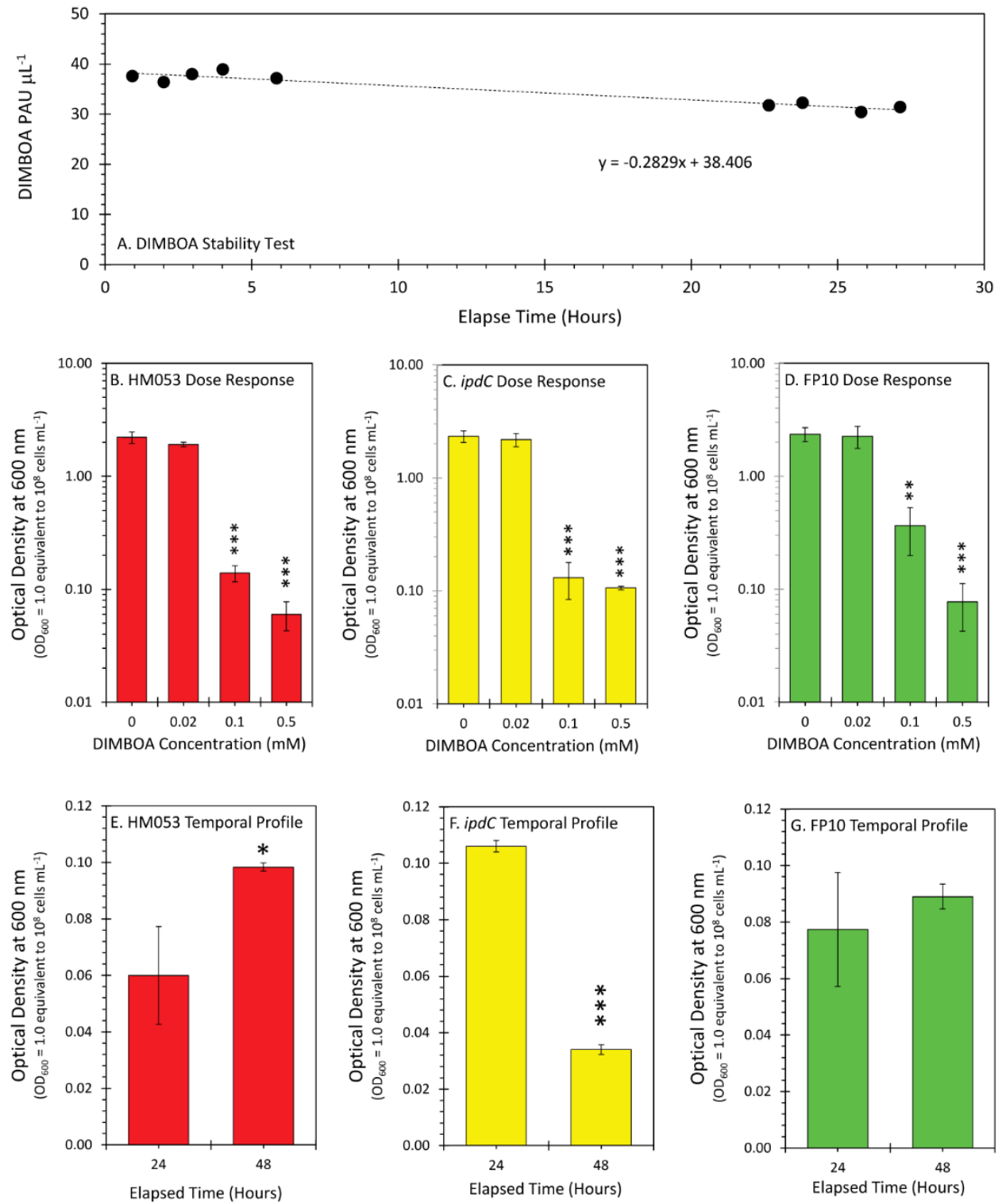

Figure 3.3.8. In vitro chemotaxis assays to examine effects of DIMBOA on Bacteria growth. Panel A: DIMBOA stability in the bacteria growth medium was examined using $0.5 \mathrm{mM}$ DIMBOA. Gas chromatography peak area units (PAU) reflecting DIMBOA levels at different time points spanning 27 hours shows a $\sim 15 \%$ loss of DIMBOA integrity. Panels B-D: DIMBOA dose response effects on bacteria growth at 24 hours as measured spectrophotometrically where $\mathrm{OD}_{600}=1$ corresponds to $10^{8} \mathrm{CFU} \mathrm{mL}^{-1}$. Panels E-G: Influence of an acute $0.5 \mathrm{mM}$ DIMBOA dose on bacteria growth after 48 hours as measured spectrophotometrically where $\mathrm{OD}_{600}=1$ corresponds to $10^{8} \mathrm{CFU} \mathrm{mL}{ }^{-1}$. Data $( \pm \mathrm{SE})$ reflected $\mathrm{N}=3$ biological replicates at each DIMBOA dose. Asterisks indicate significant differences of bacteria growth with different DIMBOA doses relative to $0 \mathrm{mM}$ DIMBOA control $(* \mathrm{P}<0.05 ; * * \mathrm{P}<0.01 ; * * * \mathrm{P}<0.001)$.

Results of the chemotaxis assay (Figure 3.3.8. B-D) revealed that all three functional mutants tolerated DIMBOA doses of $0.02 \mathrm{mM}$, but their growth was significantly reduced at higher doses. Using ${ }^{11} \mathrm{C}$ data from the present work (Figure 3.3.5. B), we used as an upper limit of root allocation of $\sim 30 \%$ fixed ${ }^{11} \mathrm{C}$ over a 3 hour period in the presence of either HM053 or 
FP10 bacteria. These same bacteria resulted in $\sim 1 \%$ fixed ${ }^{11} \mathrm{C}$ as acidic exudates over the same 3 hour period. Assuming that DIMBOA was the dominant acidic exudate, we estimate that it could account for $0.3 \%$ of the total root DIMBOA that was measured in the present work (Figure 3.3.7. D) which for HM053 and FP10 could be as high as $10 \mu \mathrm{gfw}^{-1}$ or $0.150 \mu \mathrm{M}$ accumulated over 3 hours. Adjusting for 24 hours of accumulation and for 15\% loss due to degradation, we estimate DIMBOA exudation in the present system could be as high as $1 \mu \mathrm{M}$. That said, all three functional mutants tolerated DIMBOA at approximately 20-times that level. We also conducted a temporal profile of bacteria growth testing the effects of an acute $0.05 \mathrm{mM}$ dose of DIMBOA over 48 hours (Figure 3.3.8. E-G). HM053 showed a significant increase in bacteria content even at this acute dose over 48 hours while ipdC showed a significant loss in content, and FP10 remained constant (suggesting that its rate of growth matched the rate of cell death). Hence both HM053 and FP10 functional mutants, which were shown to promote DIMBOA levels within the maize roots, appeared to tolerate the substrate.

Future studies will examine $A$. brasilense growth patterns and demand for plant-borne carbon resources as a function of different DIMBOA doses in the growth media. Recently, we published a method that describes using a combination of C-11 radiographic imaging, gamma counting and optical fluorescence imaging with RAM10 Herbaspirillum seropedicae, a green fluorescence reporting strain of an endophytic PGPB, to enable for the first time precise quantification of plant-borne carbon assimilation by a root-associating microorganism ${ }^{76}$. Efforts are underway to generate a stable fluorescent reporting strain of $A$. brasilense that will enable us to translate this method to this bacterium and test its tolerance for DIMBOA.

Altogether, the results reported herein show that inoculation of maize seedlings with HM053 A. brasilense bacteria boosts host iron assimilation and translocation to aerial portions during 
early stages of development. This behavior was seen to persist throughout the growing season having beneficial effects on plant growth that resulted in significant increases in crop yield and seed iron content ${ }^{10}$. Thus, the use of beneficial microbes not only influence plant growth, but can promote crop nutritional content.

Regarding the mechanisms of action, the data presented indicates there are strong influences of bacterial $\mathrm{BNF}$ and auxin producing capacities on $\mathrm{Fe}^{3+}$ and $\mathrm{Fe}^{2+}$ assimilation and in planta translocation. While it is enticing to suggest HM053 endows Strategy I iron capabilities in maize, the possibility cannot be ruled out that bacteria metabolism may influence the supplied iron oxidation state. However, we note that in the radiographic images taken of aerial portions from ${ }^{59} \mathrm{Fe}^{3+}$ and ${ }^{59} \mathrm{Fe}^{2+}$ exposures in HM053 inoculated plants (Figure 3.3.3. E\&F) that there was a different spatial patterning of radioisotope across the leaf surfaces. This observation suggests that the iron oxidation states were not altered by the microorganisms. Future studies, however, will examine this aspect more thoroughly using ${ }^{59} \mathrm{Fe}^{3+}$ and ${ }^{59} \mathrm{Fe}^{2+}$ coupled with ion chromatography to enable measurement of bacterial uptake and/or manipulation of the different oxidation states of iron as a function of their BNF and auxin biological capabilities.

Growth characteristics in mature maize grown outdoors- An outdoor study using potted plants enabled assessment of crop fitness to maturity and nutritional iron content in the kernel as a function of $A$. brasilense and chemical auxin treatments. Crop fitness during vegetative growth was measured in the 2019 growth season by changes in plant height and stem thickness (Figure 3.3.9.). Treatments appeared not to affect rates of plant growth as measured by plant height, but differences were seen in the thickness of the plant stems when measured at the second internode. Measurements taken on days 28 and 55 after germination showed a statistically significant increase in thickness from A. brasilense HM053-inoculated maize relative to non-inoculated 

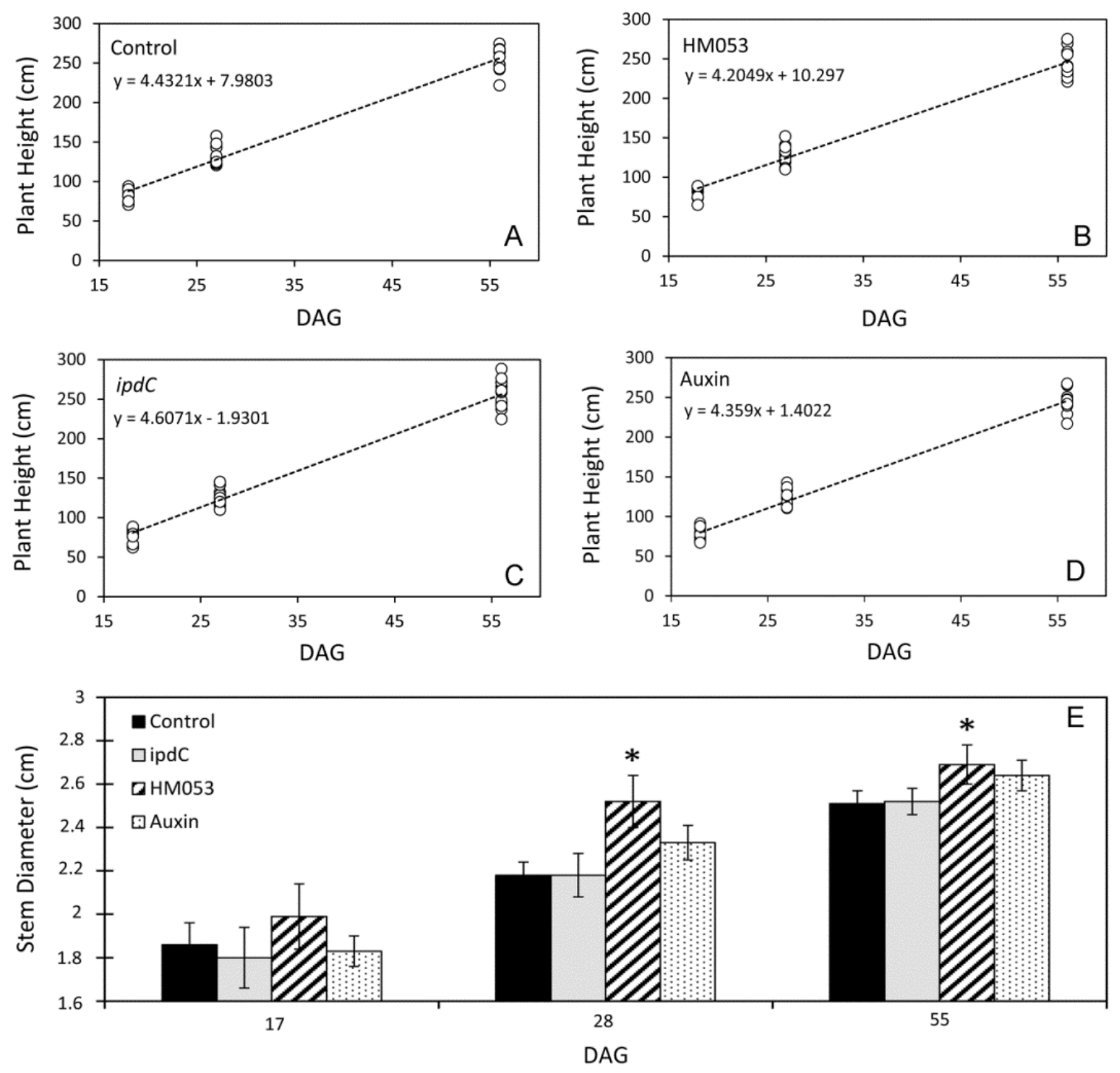

Figure 3.3.9. Plant growth rates measured as plant height and stem diameter over time. Plant heights were measured from top of soil to tallest point on shoots during the 2019 growing season spanning 17-55 days after germination (DAG). No significant difference was observed in growth rates across the treatment types. Panels (A-D): based on a comparison of growth rate slopes derived from linear regression analysis. Stem diameters were measured in centimeters at the second internode from the base of the stem. A significant increase in stem thickness was recorded for $A$.

brasilense HM053 relative to non-inoculated control maize. Data $( \pm \mathrm{SE})$ reflects $N=12$ biological replicates. Asterisks indicate significant differences $(* p<0.05)$ using Student paired t-test comparing treated to non-treated control plant stems when measured at the second internode, in which, at 28 and 55 days after germination, $A$. brasilense HM053-inoculated maize exhibited a significantly increased stem thickness relative to non-inoculated control, suggesting the biologic improved plant growth.

control while other treatments had no effect. In support of improved plant growth from $A$.

brasilense HM053 inoculation, we observed that chlorophyll content when measured in 3-week-

old lab grown seedlings (Figure 3.3.10. A) was significantly elevated from bacterial treatment 
relative to non-inoculated control plants while plants inoculated with $A$. brasilense ipdC showed a reduced leaf chlorophyll content. Though chlorophyll content was not measured in the outdoor potted plant studies, we do note that leaf thicknesses measured at the end of the 2019 growing season (Figure 3.3.10. B) were significantly greater with A. brasilense HM053 inoculation than non-inoculated controls. Our data suggests that the effects of $A$. brasilense HM053 inoculation on leaf chlorophyll in the early stages of plant growth must persist throughout the growing season since we observed improved plant growth reflected by increased stem and leaf thickness.

Finally, crop seed yield was measured as the total kernels of corn per cob (Figure 3.3.11.). It was found that maize associated with $A$. brasilense HM053 had significantly higher crop yields compared to non-inoculated control plants (34\% and 53\% increases for $A$. brasilense HM053-inoculated plants from the 2018 and 2019 growing seasons). There was also a 26\% increase in crop yield from auxin treatment in the 2018 growing season, while $A$. brasilense ipdC-associated maize exhibited a $26 \%$ lower yield than non-treated plants, though not significant, during the 2018 growing season and no difference from nontreated plants in the 2019 growing season (Figure 3.3.11.). The differences in yield between the 2018 and 2019 growing seasons
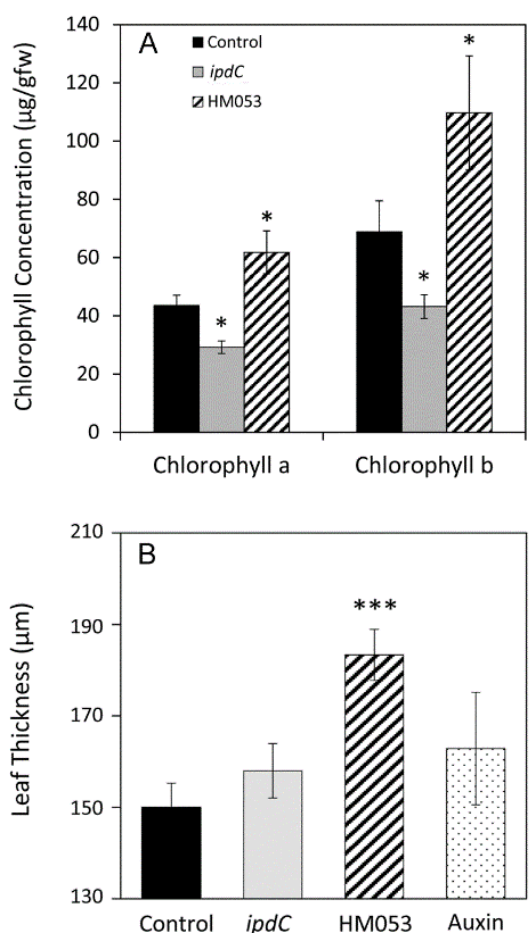

Figure 3.3.10. Leaf chlorophyll content and leaf thickness as a function of treatment type. Panel

A: The 3-week-old lab plants inoculated with Azospirillum brasilense HM053 showed significant increases in leaf chlorophyll content compared to non-inoculated control plants, while $A$. brasilense ipdC inoculated plants showed significantly reduced chlorophyll content relative to non-inoculated control plants. Panel B: Leaf thickness measurements carried out at the end of the 2019 growing season showed that plants inoculated with $A$. brasilense HM053 had significantly thicker leaves compared to control plants. Data reflects $N=12$ biological replicates in both panels for each treatment, with error bars indicating \pm SE. Paired comparisons were made using the Student $t$-test between single treatments and non-inoculated control plants. Asterisks indicate significant differences $\left({ }^{*} p<0.05\right.$, $* * * p<0.001)$ in these comparisons. 
may be attributed to differences in the outdoor environmental conditions. While plants were
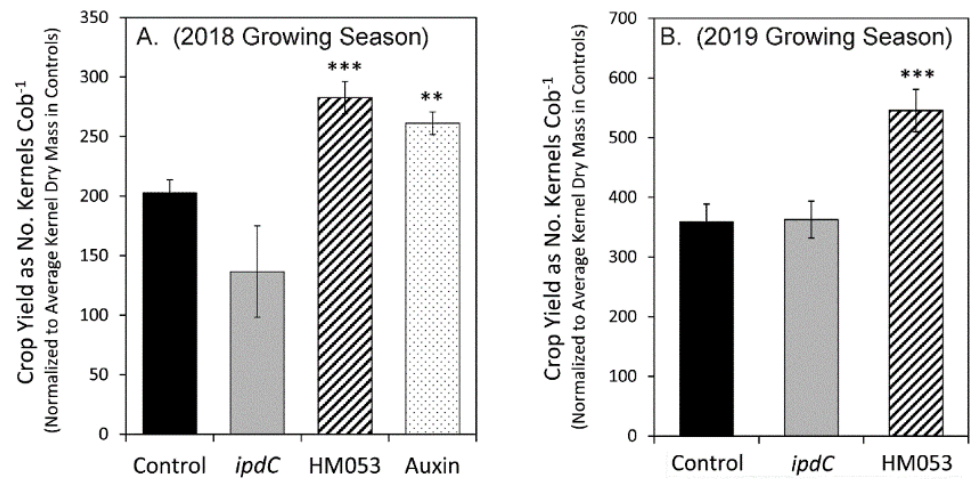

irrigated on an alternating day

cycle, there was increased rainfall

during the early portions of the

2019 growing season, which could

have contributed to the yield

Figure 3.3.11. Effect of treatment on corn seed yield. Seed yield was quantified as number of kernels per cob. In both the 2018 and 2019 seasons, plants inoculated with $A$. brasilense HM053 bacteria showed significantly enhanced kernel yields compared to control treatments. Chemical auxin treatment showed significantly enhanced kernel yield as well, while $A$. brasilense ipd $C$ and control maize were not significantly different in yield from one another. Data was normalized to the average dry kernel mass in controls and reflect $N=12$ biological replicates for each treatment. Panel A: data from the 2018 growing season; Panel B: data from the 2019 growing season). Error bars indicate \pm SE. Paired comparisons were made using the Student t-tests between single treatments and non-inoculated control plants. Asterisks indicate significant differences $(* * p<0.01, * * * p<0.001)$.

An increase in maize crop yield was noted

under $A$. brasilense HM053 inoculation and auxin

treatments; however, the main purpose of leveraging

bacterial inoculants in this study was to demonstrate a

cost-effective means to increase iron nutrition in the

crop product consumed by humans. Thus, it was most

relevant to quantify the iron content of seeds as a

function of treatment, as well as quantify the

bioavailability of that iron bound in the ferritin protein.

Ion chromatography was used to quantify total seed 
iron content as well as distinguish $\mathrm{Fe}^{2+}$ and $\mathrm{Fe}^{3+}$ oxidation states (Figure 3.3.12.). The results revealed a strong positive correlation between seed $\mathrm{Fe}^{2+}$ accumulation and total seed iron content, with $A$. brasilense HM053 generating the highest iron values. This suggests inoculation in the early stage of plant development can result in a lasting effect throughout the life cycle of the plant. Furthermore, $\mathrm{Fe}^{3+}$ showed no change as a function of inoculation. Both $A$. brasilense HM053 and ipdC data was loosely clustered at opposite ends of iron values measured in seeds (30-130 nmols iron), whereas non-inoculated control data spanned the entire range of values. Recent studies in tomato indicate early treatments with inoculums can reshape the diversity of the soil microbiome favoring dominance of the inoculum whereas non-inoculated plants can exhibit a broad spectrum of microbiome diversity ${ }^{77}$.

All kernels associated with A. brasilense HM053 or ipdC, as well as those treated with auxin, showed significant increases in kernel ferritin content (Figure

3.3.13.). This corroborates the assertion that PGPB association improves iron integration to seed, ultimately increasing its nutritional value. The auxin treatment increasing ferritin content alludes to the importance of the auxin production capabilities of the bacteria in influencing this effect, which requires further investigation. However, this does not explain the observed increase in seed ferritin content from inoculation with the auxin deficient-mutant, $A$. brasilense $i p d C$. Past perceptions have assumed that ferritin

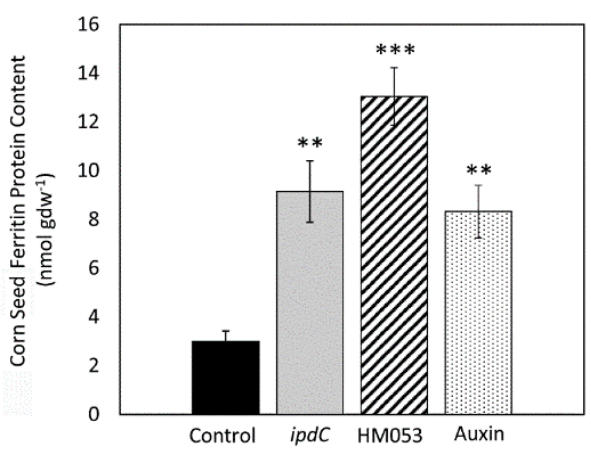

Figure 3.3.13. Seed iron-ferritin content as a function of treatment type. All treatment types showed significantly greater concentration of ferritin protein in maize seed relative to control with greatest elevation observed in the $A$.

brasilense HM053-inoculated maize kernels. Data reflects $N=4$ biological replicates for each treatment with error bars indicating \pm SE. Paired comparisons were made using the Student t-test between single treatments and non-inoculated control plants. Asterisks indicate significant differences $(* * p<0.01$, $\left.{ }^{* * *} p<0.001\right)$. 
biosynthesis is tightly regulated by cellular iron homeostasis ${ }^{78}$. Even so, the present results suggest that its regulation is even more complex.

Finally, while chemical treatments using auxin resulted in similar improvements in crop yield and crop nutritional value as $A$. brasilense HM053 inoculation relative to untreated control plants, we view chemical treatments as more intrusive to the environment, and likely a more expensive mitigation strategy than application using a liquid inoculant of bacteria.

\subsection{Materials and Methods}

Plant Growth- Maize kernels from Elk Mound Seed Co.

(Hybrid 8100) were dark germinated at room temperature for two days. Seeds were inoculated with bacteria culture as appropriate and transplanted to a growth pouch wetted with sterile Hoagland's basal salt solution (PhytoTechnology Laboratories, Shawnee Mission, KS 66215, USA) for approximately one week. Seedlings were transferred to an aeroponics system (Figure 3.4.1.), and nutrients were replenished every five days. Two week old plants were used for all the ${ }^{59} \mathrm{Fe}$ radiotracer and mass spectrometry studies.

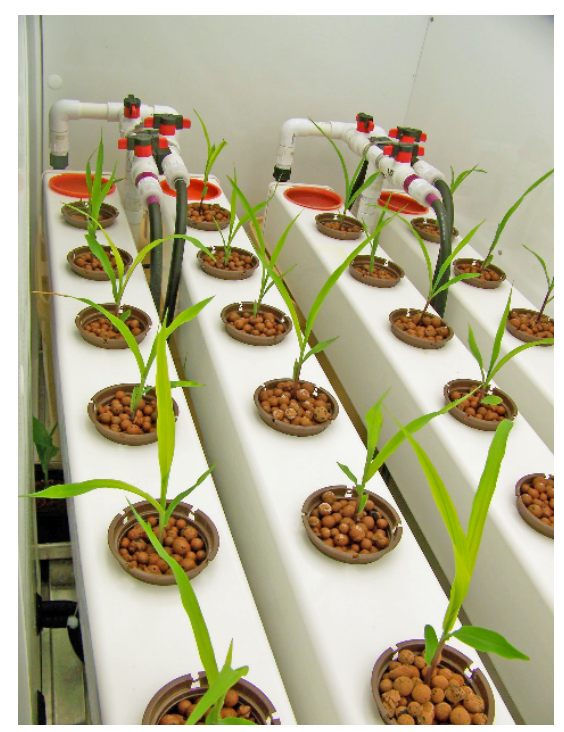

Figure 3.4.1. Maize plants raised for ${ }^{59} \mathrm{Fe}$ studies were grown aeroponically in a commercial growth chamber

For ${ }^{11} \mathrm{C}$ physiology and metabolism studies, including root emission measurements, plants were grown in eight inch plastic cones filled with Turface $^{\mathrm{TM}}$ (expanded clay matrix) where the bottom portion of the cone was immersed in de-ionized water (Figure 3.4.2.). Nutrient was introduced as Hoagland's solution every three days. Growth conditions for both set-ups consisted of 12-hour photoperiods, $500 \mu \mathrm{mol} \mathrm{m} \mathrm{m}^{-2} \mathrm{~s}^{-1}$ light intensity, and temperatures of $25^{\circ} \mathrm{C} / 20^{\circ} \mathrm{C}$ (light/dark) with humidity at $70-80 \%$ for two weeks. 


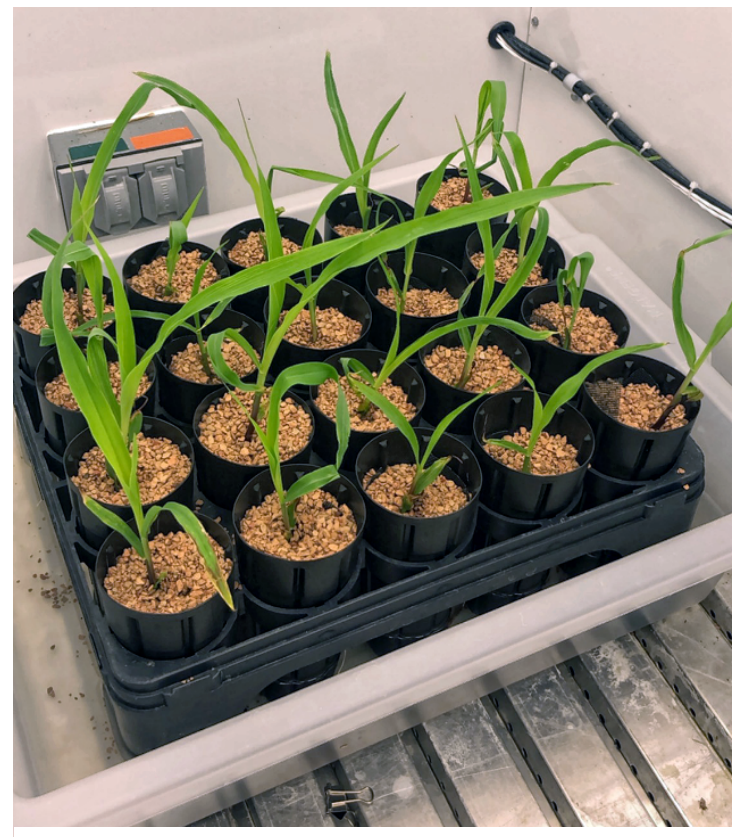

3.4.2. Maize plants raised in Turface for ${ }^{11} \mathrm{C}$ radiotracer studies.
For courtyard studies, 3 maize kernels from

Elk Mound Seed Co. (Hybrid 8100) were sown

into each of 2.7 gallon pots filled with ProMix.

After germination, any excess seedlings were

removed from each pot leaving a single plant. A

capful of fertilizer $(\sim 1.2 \mathrm{~g})$ containing nitrogen,

phosphate and potash (14-14-14, Osmocote ${ }^{\mathrm{TM}}$

Smart-Release Plant Food Flower \& Vegetable ${ }^{\mathrm{TM}}$,

The Scotts Company, Marysville, OH) was added

to the assigned pots at the time of planting.

Fertilizer was reapplied to pots 30 days after germination (DAG) and 60 DAG. Plants were placed on elevated tables outside (Figure 3.4.3.). During the 2018 growing season, four study regimes included the following: (i) non-inoculated control plants; (ii) plants inoculated with $A$.

brasilense HM053 bacterium; (iii)

plants inoculated with $A$. brasilense

ipdC bacterium; and (iv) plants

treated with $30 \mu \mathrm{M}$ auxin plant

hormone (IAA). Plants were

administered liquid inoculants at 21 ,

42 and 63 DAG. Treatments with

auxin hormone followed the same

schedule. Treatments were

randomized across the planting

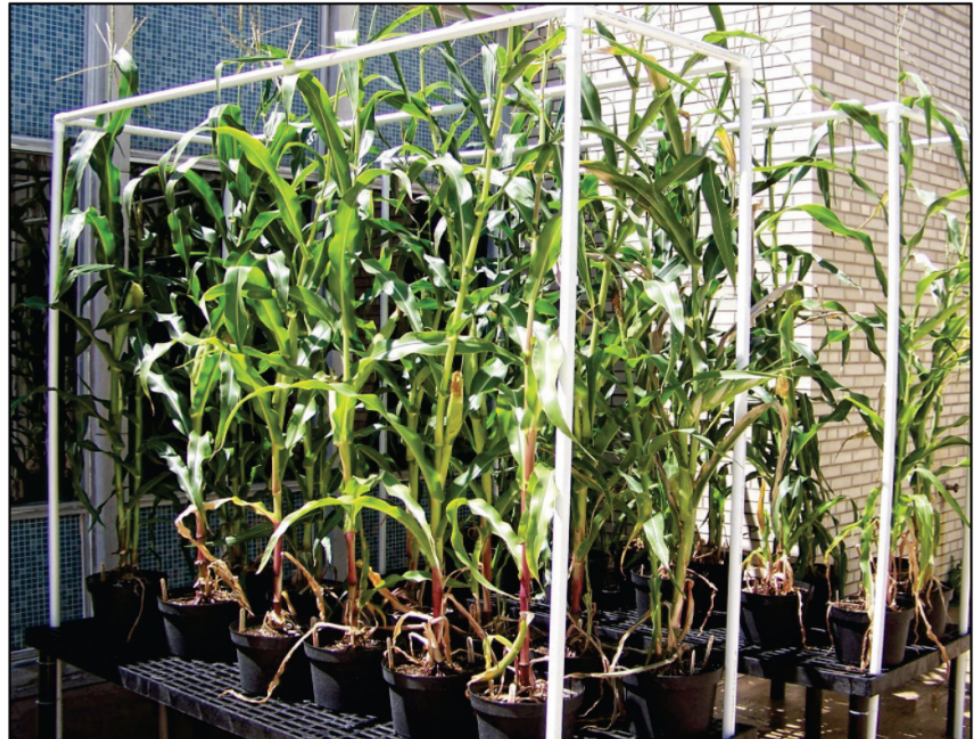

Figure 3.4.3. Outdoor potted plant studies. Plants were grown in 2.7 gallon pots in ProMix with Osmocote ${ }^{\mathrm{TM}}$ timerelease fertilizer. Treatments were randomized during the study initiation and pots were rotated weekly. 
platforms and pots were rotated weekly during the growing season. The study was repeated in the 2019 growing season with the exception that auxin treatments were not applied. Bacteria Growth and Root Inoculation- Functional mutants of $A$. brasilense were obtained via a material transfer agreement between the corresponding author's institution and the Federal University of Paraná (UFPR, Curitiba, PR CEP 81531-980, Brazil). HM053 originated as a natural mutant of the wild-type strain of $A$. brasilense FP2 (Sp7 ATCC $29145 \mathrm{Nif}^{+} \mathrm{Sm}^{\mathrm{r}} \mathrm{Nal}^{\mathrm{r}}$ ) screened through its resistance to ethylenediamine $\left(\mathrm{EDA}^{\mathrm{r}}\right)$ according to the original work of Machado et al. ${ }^{37,38}$. The FP10 mutant was obtained through the original work of Pedrosa and Yates ${ }^{39}$ by N-nitrosoguanidine mutagenesis of the FP2 wild-type strain of $A$. brasilense and isolated by growth on glutamate medium. The indole-3-pyruvate decarboxylase gene (ipdC), coding for a key enzyme of the indole-3-pyruvic acid pathway of auxin (indole-3-acetic acid) biosynthesis in Azospirillum brasilense, was functionally disrupted in a site-specific manner using a SacB-cassette insertion into the ipdC gene of wild-type FP2 (Sp7 ATCC 29145) followed by homologous recombination. The method allowed for the construction of the ipdC mutation strain without unwanted sequence changes and relied on the $\lambda$ Red recombineering (Direct and Inverted Repeat stimulated excision; DIRex) a method that works well for generating single point mutations, small insertions or replacements as well as deletions of any size, in a bacterial gene $^{40}$. The resultant knock-out strain exhibited significant reduction in auxin biosynthesis to a level of $10 \%$ that of the wild-type $\operatorname{strain}^{41}$.

Functional mutants were grown in liquid NFbHP-lactate medium following published procedures $^{3}$. Cultures were washed with sterile water and diluted to approximately $10^{6}$ to $10^{8}$ colony forming units (CFU) per $\mathrm{mL}$ volume. Root inoculation involved adding $1 \mathrm{~mL}$ of inoculum to a Petri dish containing 10 maize seedlings ( 2 days after germination) and rocking in 
the shaking incubator for two hours. Seeds were placed into plastic seed germination pouches (PhytoAB, Inc., San Jose, CA 95131, USA) for five days before transplanting to aeroponics or Turface $^{\mathrm{TM}}$. For the outdoor grown maize inoculation, bacterial cultures were grown in $25 \mathrm{~mL}$ flasks in a shaking incubator set to $30^{\circ} \mathrm{C}$ and $130 \mathrm{rpm}$ until $\mathrm{OD}_{600}=1.0$ (Optical Density at 600 $\mathrm{nm}$, corresponding to $10^{8}$ cells $\mathrm{mL}^{-1}$ ) was reached. Bacterial cultures were washed with sterile water and diluted to approximately $10^{6}$ to $10^{8}$ colony forming units (CFU). Bacterial culture (10 $\mathrm{mL}$ ) was injected into the soil at the base of the stem at the previously specified 3 time points during the growing season. Verification of bacteria presence at harvest was not carried out. Bacteria Quantification, Drop Plate Assay- Bacterial quantifications were performed concurrently with metabolite studies. Plants were harvested from the growth media and rinsed in DI water. Two separate 1-1.5 inch sections of primary root growth were taken from each plant and weighed (approximately 100-300 mg total). The sample was ground with mortar and pestle in $1 \mathrm{~mL}$ of $1 \%$ saline. Five serial dilutions were performed; the first with $100 \mu \mathrm{L}$ of the ground extract into $900 \mu \mathrm{L}$ of $1 \%$ saline and each subsequent dilution being $100 \mu \mathrm{L}$ of the previous dilution into $900 \mu \mathrm{L}$ of $1 \%$ saline. Each serial dilution was plated in triplicate by $10 \mu \mathrm{L}$ drops onto agar plates fortified with lactate growth media and incubated at $30{ }^{\circ} \mathrm{C}$ for $48-72 \mathrm{hr}$. before counting. The dilution that contained 3-40 colony forming units (CFU) per $10 \mu \mathrm{L}$ drop was counted and used to perform calculations (Figure 3.3.2.).

Radioactive Fe-59 Tracer Studies- Two oxidation states of radioactive ${ }^{59} \mathrm{Fe}\left(\mathrm{t}_{1} / 24.5 \mathrm{~d}\right)$ were used in the study, ferrous $\left(\mathrm{Fe}^{2+}\right)$ and ferric $\left(\mathrm{Fe}^{3+}\right)$, purchased from Perkin-Elmer Life Sciences (Akron, OH USA). One hour before administration of tracer, plants were removed from aeroponics growth cells and placed in $600-\mathrm{mL}$ beakers with $100 \mathrm{~mL}$ of $5 \mu \mathrm{M} \mathrm{Fe}^{3+}$-EDTA to prohibit induction of water- or iron-stress responses during the study. In auxin treatment studies, $50 \mathrm{~mL}$ 
of $30 \mu \mathrm{M}$ indole-3-acetic acid (auxin) was added to $50 \mathrm{~mL}$ of the iron solution for a total of 100 $\mathrm{mL}$. Iron radiotracer of $0.74 \mathrm{MBq}$ was introduced. After three hours, the roots were cut from the shoots and washed in water followed by an EDTA solution, then blotted dry. The root and shoot tissues were counted in a three inch NaI (PMT: Photomultiplier Tube) gamma well-type detector for percent assimilation and allocation calculations.

Production and Administration of Radioactive $\left[{ }^{11} \mathrm{C}_{\mathrm{CO}_{2}-}\left[{ }^{11} \mathrm{C}_{\mathrm{CO}_{2}}\left(\mathrm{t} \frac{1}{2} 20.4 \mathrm{~min}\right)\right.\right.$ was produced on the GE PETrace Cyclotron located at the Missouri Research Reactor Center using high-pressure research grade $\mathrm{N}_{2}$ gas target irradiated with a $16.4 \mathrm{MeV}$ proton beam to generate ${ }^{11} \mathrm{C}$ via the ${ }^{14} \mathrm{~N}(\mathrm{p}, \alpha){ }^{11} \mathrm{C}$ nuclear transformation ${ }^{42,43}$ using our published procedures ${ }^{44}$. The $\left[{ }^{11} \mathrm{C}_{\mathrm{CO}_{2}}\right.$ was trapped on the molecular sieve, desorbed, and quickly released into an air stream at $200 \mathrm{~mL} \mathrm{~min}-1$ as a discrete pulse for labeling a leaf affixed within a $5 \times 10 \mathrm{~cm}$ lighted $(560$ $\mu \mathrm{mol} \mathrm{m} \mathrm{s}^{-2} \mathrm{~s}^{-1}$ ) leaf cell to ensure a steady level of fixation. The load leaf affixed within the cell was pulse-fed ${ }^{11} \mathrm{CO}_{2}$ for $1 \mathrm{~min}$, then chased with normal air for the duration of exposure. A PIN diode radiation detector (Carroll Ramsey Associates, Berkeley, CA 94710, USA) attached to the bottom of the leaf cell enabled continuous measurement of radioactivity levels within the cell during the initial pulse and in the minutes directly following to give information on ${ }^{11} \mathrm{CO}_{2}$ fixation and leaf export of ${ }^{11} \mathrm{C}$-photosynthates ${ }^{44}$. Whole-Plant ${ }^{11} \mathrm{C}$-Physiology Measurements- After pulse labeling using $\left[{ }^{11} \mathrm{C}\right] \mathrm{CO}_{2}$, plants were incubated for three hours before separating the load leaf, shoots, roots and growth media. Roots were washed with $100 \mathrm{~mL}$ of de-ionized water to remove surface root exudates. Measurement of ${ }^{11} \mathrm{C}$-activity was performed using gamma counting and data was decay corrected to end-ofbombardment. The individual components (plant tissues, growth media and root wash) were summed for total plant ${ }^{11} \mathrm{C}$-activity. Individual components were used to calculate leaf export, 
root allocation and root exudation fractions. Aliquots of the root washings were processed through a $1 \mathrm{~mL}$ column packed using 200 mesh AG1 X-8 anion exchange resin (Bio-Rad Laboratories, Inc., Hercules, CA, USA). Afterward, the column and breakthrough rinse were counted for radioactivity providing a measure of the acidic and non-acidic fractions of root exudates.

$\left[{ }^{11 / 12} \mathrm{C}\right]$ Metabolite Analyses- In separate studies, leaves were exposed to $\left[{ }^{11} \mathrm{C}\right] \mathrm{CO}_{2}$ for twenty minutes before metabolite analyses following published procedures ${ }^{45}$. Tissues were flash frozen in liquid nitrogen, ground to a fine powder and extracted in methanol: water $(60: 40 \mathrm{v} / \mathrm{v})$ in Eppendorf ${ }^{\mathrm{TM}}$ tubes. After centrifugation the insoluble and soluble portions were separated and counted for ${ }^{11} \mathrm{C}$-activity using a $\mathrm{NaI}(\mathrm{Tl})$ gamma counter. The insoluble portion contained mostly cell-wall polymers and starch. The soluble portion contained small water-soluble compounds, including sugars, amino acids and non-nitrogen containing organic acids. All data was decay corrected back to the end-of-bombardment.

$\left[{ }^{11} \mathrm{C}\right]$-Sugars were analyzed by radio thin layer chromatography (TLC) using glass backed $\mathrm{NH}_{2}$-silica HPTLC-plates $(200 \mu \mathrm{m}, \mathrm{w} / \mathrm{UV} 254)$ purchased from Sorbent Technologies (Atlanta, GA, 30071, USA) according to published procedures ${ }^{46,47}$. In the same method, the $\left[{ }^{12} \mathrm{C}\right]$-sugars could be measured after thermal reaction of the carbohydrates with amino groups according to prior published work ${ }^{45}$. TLC plates were spotted with sugar standards using a semi-automatic Linomat 5 sample applicator (Camag Scientific Inc., Wilmington, NC, 28401, USA) for correlation of biological samples to absolute concentration and developed using a mobile phase consisting of 65:20:15 Acetonitrile: Methanol: Deionized Water (v/v). The TLC plates were imaged using autoradiography and activity in each spot was quantified using ImageQuant TL 7.0 software. Total $\left[{ }^{11} \mathrm{C}\right]$-sugar content was related to the ${ }^{11} \mathrm{C}$-activity quantified along the sample 
lane of the TLC plate, and then corrected to percent total fixed $\left[{ }^{11} \mathrm{C}\right] \mathrm{CO}_{2}$ using gamma count data from the insoluble and soluble fractions.

$\left[{ }^{11} \mathrm{C}\right]$-Amino acids were analyzed following our published procedures ${ }^{45}$ using pre-column OPA derivatization and quantified by gradient radio HPLC (Sonntek, Inc. Upper Saddle River, NJ 07458, USA) using a Phenomenex X-Bridge $5 \mu \mathrm{m} \mathrm{C18} \mathrm{(150} \mathrm{mm} \mathrm{x} 4.6 \mathrm{~mm}$ inner diameter) column heated to $30{ }^{\circ} \mathrm{C}$ and mobile phase system comprised of Solvent A (water, $\mathrm{pH}$ 5.75), Solvent B (0.01 $\left.\mathrm{M} \mathrm{NaH}_{2} \mathrm{PO}_{4}, \mathrm{pH} 6.8\right)$ and solvent $\mathrm{C}$ (methanol) starting at 75:25 of A:B at injection and ramping to $20: 80$ of $\mathrm{B}: \mathrm{C}$ within 30 minutes at a flow rate of $0.7 \mathrm{~mL} \mathrm{~min}^{-1}$. On-line fluorescence detection (340 nm excitation, $450 \mathrm{~nm}$ emission; Hitachi LaChrom Elite L-2485; Sonntek, Inc.) was used for quantification of the OPA-derivatized $\left[{ }^{12} \mathrm{C}\right]$ amino acids and a $\mathrm{NaI}$ (PMT) gamma radiation detector (Ortec, Inc.) enabling direct measurement of $\left[{ }^{11} \mathrm{C}\right]$-labelled amino acids. Data was acquired using PeakSimple ${ }^{\mathrm{TM}}$ chromatography software v4.88 (SRI, Inc., Torrance, CA 90503, USA) and compared to standard calibration curves constructed for each amino acid giving absolute amounts for individual metabolites in units of $\mu \mathrm{mol}$ per gram fresh weight $\left(\mathrm{gfw}^{-1}\right)$ tissue. Radioactive metabolite peaks were quantified using the same software, corrected for radioactive decay and related back to absolute amounts of fixed ${ }^{11} \mathrm{C}$ activity in the plant at the start of the study.

For $\left[{ }^{11} \mathrm{C}\right]$-organic acid analysis, leaf extract was processed through a QMA Sep-Pak ${ }^{\mathrm{TM}}$ then rinsed with $10 \mathrm{~mL}$ of deionized (DI) water followed by $1 \mathrm{~mL}$ of $20 \mathrm{mM}$ sodium phosphate buffer $(\mathrm{pH}=1.5)$. Aliquots of the phosphate rinse were analyzed using a gradient radio HPLC equipped with a UV detector $(210 \mathrm{~nm})$ and an on-line $\mathrm{NaI}(\mathrm{Tl})$ radiation detector. The acids were separated using a Phenomenex Gemini $5 \mu \mathrm{m} \mathrm{C18}(250 \times 4.6 \mathrm{~mm}$ inner diameter $)$ column with a solvent system of solvent A (20 mM potassium phosphate monobasic $\mathrm{pH}$ of 2.5$)$ and solvent $\mathrm{B}(60: 40$ 
methanol: acetonitrile: (v/v)) starting at 100\% A at injection and ramping to 75:25 A:B within 10

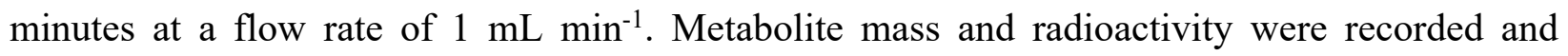
quantified as described above for amino acids.

$\left[{ }^{11} \mathrm{C}\right]-$ Nicotianamine was analyzed by reacting a portion of the QMA extract with $10 \mathrm{mM}$ ferrous sulfate heptahydrate $(1: 1 \mathrm{v} / \mathrm{v})$ for 30 minutes to chelate the nicotianamine. A radio-HPLC system was used in the analysis where $20 \mathrm{mM}$ potassium phosphate buffer at $\mathrm{pH} 2.50$ (solvent $\mathrm{A}$ ) and 60:40 methanol:acetonitrile (solvent B) were pumped through a Phenomenex Gemini C18 110A 250 x $4.60 \mathrm{~mm}$ column. The solvent gradient changed from 100\% solvent A to $75 \%: 25 \%$ $\mathrm{A}: \mathrm{B}$ at 10 minutes, and back to $100 \%$ A by 20 minutes where it was held for 5 minutes. The chelated sample was measured by UV absorption at $243 \mathrm{~nm}$. The amount of nicotianamine mass per gram fresh weight tissue was assessed for control, HM053, FP10 and ipdC treatments using authentic nicotianamine standards to calibrate the detector response. Radioactivity associated with this metabolite was measured by $\mathrm{NaI}(\mathrm{Tl})$ detector connected in series with the $\mathrm{UV}$ detector.

Specific activities (SA) of individual metabolites of interest including $\left[{ }^{11} \mathrm{C}\right]$-histidine, $\left[{ }^{11} \mathrm{C}\right]-$ citric acid and $\left[{ }^{11} \mathrm{C}\right]$-nicotianamine were calculated using Equation 3.4.1.:

$$
\mathrm{SA}=\left(\% \text { Fixed }{ }^{11} \mathrm{C} \text { Activity }\right) /\left(\mu \text { moles of metabolite mass } \times \mathrm{gfw}^{-1} \text { tissue }\right) \quad \text { [3.4.1.] }
$$

Raw radioactivity counts were decay corrected back in time to the end of the ${ }^{11} \mathrm{C}$ cyclotron target irradiation using Equation 3.4.2.:

$$
\mathrm{A}_{\mathrm{o}}=\mathrm{A}_{\mathrm{T}} \times \exp (\lambda \mathrm{T})
$$

where $\mathrm{A}_{\mathrm{o}}$ is the calculated decay corrected radioactivity at $\mathrm{T}_{\mathrm{o}}$ or $\mathrm{EOB}, \mathrm{A}_{\mathrm{T}}$ is the measured radioactivity at time $\mathrm{T}, \lambda$ is the decay constant equal to $\left(\ln 2 / \mathrm{t}_{1} / 2\right)$ where $\mathrm{t}_{1 / 2}$ is the half-life for ${ }^{11} \mathrm{C}$ equal to 20.4 min., and $\mathrm{T}$ is the elapsed time from EOB to when the sample was counted. PeakSimple peak integrals for radioactivity amounts were correlated to disintegrations per 
minute units of absolute radioactivity using a cross calibration factor to relate the HPLC detector response to that of the static $\mathrm{NaI}(\mathrm{Tl})$ gamma counter. Metabolite masses in $\mu$ moles were calculated from PeakSimple mass peak integrals using the mass detector response to authentic standards.

$\left[2-{ }^{11} \mathrm{C}\right]-$ Indole and its Application to Measuring Microbial Auxin Biosynthesis- Radiosynthesis of 2-[ $\left.{ }^{11} \mathrm{C}\right]$-indole followed published procedures using $\left[{ }^{11} \mathrm{C}\right]-\mathrm{HCN}^{48}$. Synthesis took less than 1 hour providing product at $>98 \%$ radiochemical purity, and a specific activity of $176 \pm 24.8 \mathrm{GBq}$ $\mathrm{mmol}^{-1}$.

Aliquots of purified radiotracer were dispensed into $10 \mathrm{~mL}$ Falcon tubes containing thoroughly washed and harvested bacteria re-suspended in sterile water. Bacteria counts were measured by sample turbidity, where $\mathrm{OD}_{600}=1.0$ (Optical Density at $600 \mathrm{~nm}$, corresponded to $10^{8}$ cells $\left.\mathrm{mL}^{-1}\right)$. Once tracer was introduced, tubes were gently rocked at ambient temperature for up to 2 hours and samples were periodically removed for analysis of $2-\left[{ }^{11} \mathrm{C}\right]$ indole- 3 -acetic acid conversion.

2-[ $\left[{ }^{11} \mathrm{C}\right]$ indole-3-acetic acid analysis was carried out using radio HPLC using a Phenomenex Gemini $5 \mu \mathrm{m} \mathrm{C} 18(250 \times 4.6 \mathrm{~mm}$ inner diameter $)$ column at $35^{\circ} \mathrm{C}$ and a mobile phase composed of acetonitrile:water $(50: 50, \mathrm{v} / \mathrm{v} \%)$ at flow rate of $1.0 \mathrm{~mL} \mathrm{~min}^{-1}$. The fluorescence detector was set with excitation and emission wavelengths of $230 \mathrm{~nm}$ and $360 \mathrm{~nm}$, respectively with a $\mathrm{NaI}(\mathrm{Tl})$ gamma radiation detector in series.

Autoradiography-Subsequent to Fe-59 exposures plants were harvested, roots were dried and radiographic images of different tissue areas (roots and shoots) were acquired by exposing phosphor plate films for up to 16 hours. Phosphor plates were read using the Typhoon 9000 
imager (Typhoon ${ }^{\mathrm{TM}}$ FLA 9000, GE Healthcare, Piscataway, NJ 08854, USA). Images were used qualitatively for determining spatial patterning of tracer in roots and shoots.

Inductively Coupled Plasma-Mass Spectrometry (ICP-MS)- For ICP-MS analyses, roots and shoots were dried in an oven, weighed into digestion vessels and digested in $3.0 \mathrm{~mL}$ of concentrated nitric acid at $190^{\circ} \mathrm{C}$ in a Milestone Ethos Plus (Milestone SRL, Sorisole, Italy) microwave digestion system. Digestants were diluted to $50 \mathrm{~mL}$ with ultrapure water and gravimetrically diluted by a factor of 10 with $0.45 \mathrm{M}$ nitric acid. Samples were analyzed via Perkin-Elmer NexION ICP-MS in Kinetic Energy Discrimination mode. Total elemental ion counts were measured of ${ }^{56} \mathrm{Fe}$ and normalized to ${ }^{12} \mathrm{C}$ counts. Reference materials included NIST SRM 1570 spinach leaves and NIST SRM 1573 tomato leaves prepared as samples were. Internal Calibration standards of $\mathrm{Sc}$, In, and $\mathrm{Tl}$ at known concentrations were used from stock solutions (High Purity Standards, Charleston, SC 29418, USA).

For Laser Ablation-ICP-MS, roots were removed from shoots and a 3-cm section of primary lateral root was further excised above the apical meristem. Roots were sectioned in OCT to 100 $\mu \mathrm{m}$ thickness (Fisher Scientific Inc., Hampton, NH 03801, USA) and placed on quartz microscope slides for freeze drying in a FreezeZone 1 dryer (Labconco Corp., Kansas City, MO, USA) before ablation.

Transmission Electron Microscopy- Specimens were collected from control and inoculated groups and processed for transmission electron microscopy (TEM). Unless otherwise stated, all reagents for electron microscopy were purchased from Electron Microscopy Sciences (EMS, Inc., Hatfield, PA 19440, USA) and all specimen preparation was performed at the Electron Microscopy Core Facility, University of Missouri. Tissues were fixed in 2\% paraformaldehyde, $2 \%$ glutaraldehyde in $100 \mathrm{mM}$ sodium cacodylate buffer $\mathrm{pH}=7.35$. Fixed tissues were rinsed 
with $100 \mathrm{mM}$ sodium cacodylate buffer, $\mathrm{pH} 7.35$ containing $130 \mathrm{mM}$ sucrose. Secondary fixation was performed using $1 \%$ osmium tetroxide solution containing $1.5 \%$ potassium ferrocyanide in cacodylate buffer using a Pelco Biowave (Ted Pella, Inc., Redding, CA 96003, USA) operated at 100 Watts for 1 minute. Specimens were next incubated at $4{ }^{\circ} \mathrm{C}$ for 1 hour, then rinsed with cacodylate buffer and distilled water. En bloc staining was performed using 1\% aqueous uranyl acetate and incubated at $4{ }^{\circ} \mathrm{C}$ overnight, then rinsed with distilled water. A graded dehydration series was performed using ethanol, transitioned into acetone, and dehydrated tissues were then infiltrated with a 1v/1v of Epon and Spurr resin for 24 hours at room temperature and polymerized at $60^{\circ} \mathrm{C}$ overnight. Sections were cut to a thickness of $80 \mathrm{~nm}$ using an ultramicrotome (Ultracut UCT, Leica Microsystems, Germany) and a diamond knife (Diatome, Hatfield PA 19440, USA). Thin sections were stained using Reynold's Lead Citrate. Images were acquired with a JEOL JEM 1400 transmission electron microscope (JEOL, Peabody, MA 01960, USA) at $80 \mathrm{kV}$ on a Gatan Ultrascan 1000 CCD (Gatan, Inc., Pleasanton, CA 94588, USA).

Root $p H$ Visualization- A solution of Hoagland's nutrient salt $\left(1.608 \mathrm{~g} \mathrm{~L}^{-1}\right)$ with MES $\left(0.55 \mathrm{~g} \mathrm{~L}^{-}\right.$ ${ }^{1}$ ) was prepared in de-ionized $\mathrm{H}_{2} \mathrm{O}$ and adjusted to a $\mathrm{pH}$ of 6.0 with $30 \% \mathrm{KOH}$. Gelrite ${ }^{\mathrm{TM}}$ was added $\left(2.8 \mathrm{~g} \mathrm{~L}^{-1}\right)$ and the resulting solution was autoclaved. Once autoclaved, bromothymol blue $\left(0.1196 \mathrm{~g} \mathrm{~L}^{-1}\right)$ was added. Maize seedlings were germinated as described and transplanted to glass cells containing the dye enriched gel mixture. After five days of growth seedlings were removed and imaged with a digital camera (Figure 3.3.6.).

Root Ethylene Emission Measurements- Aeroponically grown plants were harvested at two weeks, roots weighed and placed in air-tight jars equipped with a sampling port. Roots from 2-3 plants were binned generating a single data point. Roots were volatilized for 90-130 hours and 5- 
$\mathrm{mL}$ of head space was sampled using a gas-tight syringe. The sample was injected into a flameionization Hewlett Packard 5890A gas chromatograph with a 2-m long, 1-mm inner diameter ShinCarbon ST packed column. The program started with a two minute hold at $40^{\circ} \mathrm{C}$ and increased $10{ }^{\circ} \mathrm{C} \mathrm{min}-1$ to $250{ }^{\circ} \mathrm{C}$. The injector temperature was $250{ }^{\circ} \mathrm{C}$ and the flame ionization detector temperature was $300{ }^{\circ} \mathrm{C}$. Chromatographic peaks for ethylene were measured using PeakSimple ${ }^{\mathrm{TM}}$ chromatography software and quantified against ethylene standards. Ethylene emission values were reported as pmol ethylene $\mathrm{gfw}^{-1}$ tissue $\mathrm{hr}^{-1}$ as a function of bacteria treatment.

Root Gravitropism Measurements- Maize seeds were placed upright in Gelrite ${ }^{\mathrm{TM}}$ solid media in a growth box. The box was rotated $90^{\circ}$ after root growth reached half way down the box in the Gelrite $^{\mathrm{TM}}$ media. Photographs of the roots were taken after twelve hours. Root bending angles were measured with Image-J software. The degree of bending as a function of bacterial treatment was assessed as an indirect measurement of plant-root auxin status.

Root Indole Measurements- Fresh roots were harvested from two week old plants, weighed, and placed in air-tight jars equipped with two sampling ports. The roots volatilized for 48-90 hours. A glass tube trap was loaded with $50 \mathrm{mg}$ of Tenax ${ }^{\circledR} \mathrm{GR}$, a composite mix of Tenax ${ }^{\circledR}$ TA (Scientific Instrument Services, Inc., NJ 08551, USA), a porous polymer based on 2, 6diphenylphenol with $30 \%$ graphite additive. This trap was affixed to one port equipped with a silicon rubber seal and an aquarium air pump was affixed to the other port. Air was pumped through the chamber for one hour at a rate of $50 \mathrm{~mL} \mathrm{~min}^{-1}$ providing three complete air exchanges. Tenax ${ }^{\circledR}$ traps were rinsed with $0.5 \mathrm{~mL}$ of methylene chloride and 1-3 $\mu \mathrm{L}$ volumes of this extract were analyzed by capillary gas chromatography using a Hewlett Packard 5890A gas chromatograph equipped with a $30-\mathrm{m}$ long, $0.53 \mathrm{~mm}$ inner diameter, $0.25 \mu \mathrm{m}$ film thickness, 
Restek RTX-WAX column for separation and a flame ionization detector. The program was as described for ethylene measurements. Chromatographic peaks for indole eluted at 15 minutes and were measured using PeakSimple chromatography software and quantified against standards. Root indole volatile emission rates were reported as $\rho$ mole indole $\mathrm{gfw}^{-1} \mathrm{hr}^{-1}$ as a function of bacteria treatment.

Root DIMBOA Measurements- Maize root tissue $(700-800 \mathrm{mg})$ was macerated by mortar and pestle in $2.00 \mathrm{~mL}$ of DI water. The resulting extract was centrifuged, combined with $1 \mathrm{~mL}$ of ethyl acetate (ETAC) and gently rocked for one minute. The DIMBOA/ETAC organic layer was removed and the aqueous phase was re-extracted with $1 \mathrm{~mL}$ of ETAC and combined with the first organic sample. An aliquot of the organic phase was injected into a Hewlett Packard 5890A gas chromatograph equipped with a $30-\mathrm{m}$ long, $0.53 \mathrm{~mm}$ inner diameter, $0.25 \mu \mathrm{m}$ film thickness, Restek RTX-WAX capillary column and flame ionization detector. The program started with a two minute hold at $70{ }^{\circ} \mathrm{C}$ and increased $10{ }^{\circ} \mathrm{C} \mathrm{min}{ }^{-1}$ to $250^{\circ} \mathrm{C}$. The injector temperature was 250 ${ }^{\circ} \mathrm{C}$ and the flame ionization detector temperature was $300{ }^{\circ} \mathrm{C}$. The retention time of DIMBOA was approximately 24 minutes. Chromatographic peaks for DIMBOA were measured using PeakSimple chromatography software and quantified against authentic standards (Santa Cruz Biotechnology, Inc., TX, USA). Root DIMBOA values were reported as $\mu$ mole DIMBOA $\mathrm{gfw}^{-1}$ tissue as a function of bacteria treatment.

In vitro Chemotaxis Assays to Examine Effects of DIMBOA on Bacteria Growth- A standard of DIMBOA was prepared by dissolving $10 \mathrm{mg}$ of compound in $6 \mathrm{~mL}$ of deionized sterile water using $200 \mu \mathrm{L}$ of DMSO (3\% v/v) to facilitate sample dissolution. Aliquots of this standard were then added to bacteria growth media (as described in Bacteria Growth Methodology section) to generate $0.02 \mathrm{mM}, 0.1 \mathrm{mM}$ and $0.5 \mathrm{mM}$ concentrations of DIMBOA. Each dose was replicated in 
triplicate. The amount of DMSO for each dose was adjusted to match that of the highest DIMBOA concentration. Additionally, $0 \mathrm{mM}$ DIMBOA controls were prepared (also in triplicate) where an equivalent amount of DMSO as that found in the highest $0.5 \mathrm{mM}$ DIMBOA dose was introduced to null effects of this organic solvent on bacteria growth. A. brasilense cultures of the functional mutants HM053, ipdC and FP10 were introduced into these modified media and grown under previously described conditions for 24 hours after administration of DIMBOA to the growth media. An additional sample of media containing $0.5 \mathrm{mM}$ DIMBOA, but no bacteria, was set aside for examining DIMBOA stability at $30^{\circ} \mathrm{C} . \mathrm{OD}_{600}$ measurements were taken using a ThermoScientific Evolution $201 \mathrm{UV}$-vis spectrophotometer at 24 hours into the bacteria growth cycle. The $0.5 \mathrm{mM}$ DIMBOA cultures were re-measured after 48 hours of incubation. DIMBOA stability measurements were conducted over 27 hours using capillary gas chromatography and flame ionization detection using the analytical methodology previously described above. Samples were prepared for GC injection by extracting $200 \mu \mathrm{L}$ of growth media using $500 \mu \mathrm{L}$ of ETAC. Aliquots of the organic extract were then injected into the gas chromatograph using a 1:20 sample split upon injection.

Plant Growth Measurements- Maize plants were measured with a tape measure from the soil of the pot up to the highest point of the plant in centimeters. They were measured at three time points: 17,28 , and 55 days after germination. Stem diameters were measured at the second internode up from the base of the stem using calibers. Tape was applied at these stem locations after the first time point measurement which served as a visual marker for subsequent measurements.

Chlorophyll and Leaf Thickness Measurements- Vegetative tissues were separated from roots and placed in pre-weighed and pre-chilled Eppendorf ${ }^{\mathrm{TM}}$ tubes. They were flash frozen in liquid 
nitrogen, ground to a fine powder and weighed. A volume of acetone equivalent to four times the milligram mass of the frozen ground tissue (assuming uniform densities) was added to the centrifuge tube. The content was vortexed (VWR analog vortex mixer; Sigma-Aldrich Corp., St. Louis, MO, USA) then sonicated (Branson Bransonic 32; Sigma-Aldrich Corp. St. Louis, MO, USA) at $0{ }^{\circ} \mathrm{C}$ for 2 minutes. Additional vortexing was performed during this period to ensure complete mixing. The Eppendorf ${ }^{\mathrm{TM}}$ tubes were then centrifuged for 2 minutes at 15,000 rpm to separate the insoluble and soluble portions. The insoluble portion contained mostly cell-wall polymers and starch while the soluble portion contained small soluble compounds such as sugars. The liquid extract was spotted onto a silica TLC plate in a $5 \mathrm{~mm}$ band using $2 \mu \mathrm{L}$ of sample. The silica plate was developed in a 6:1.6:1:0.4 ratio of petroleum ether: hexane: acetone: methanol solution. Once the plate was fully developed, a razor was used to scrape off bands containing chlorophyll a and chlorophyll $\mathrm{b}$. The shavings were placed in two separate Eppendorf ${ }^{\mathrm{TM}}$ tubes. Acetone $(1 \mathrm{~mL})$ was added to the tube containing chlorophyll a and the tube containing chlorophyll $\mathrm{b}$. The samples were then vortexed and centrifuged for 4 minutes at $15,000 \mathrm{rpm}$. The absorbance of the resulting supernatant of each sample was analyzed via ultraviolet-visible spectroscopy (UV-VIS). Chlorophyll a was recorded at a wavelength of 663 $\mathrm{nm}$, and Chlorophyll $\mathrm{b}$ was measured at a wavelength of $645 \mathrm{~nm}$. Beer's Law $(\mathrm{A}=\varepsilon \mathrm{bc})$ was then utilized to determine sample concentration of chlorophyll. Precision calipers (Fowler High Precision, Newton, MA, USA) were used to determine leaf thickness.

Ion Chromatography Measuring Seed Iron- Ferric, ferrous, and total iron were quantified from corn kernels with ion chromatography coupled with chemiluminescence detection ${ }^{79}$ following the collection and drying of the kernels in an oven for 3 weeks at $65^{\circ} \mathrm{C}$. Seeds were pulverized between plastic sheets using a wooden mallet and dissolved in $1 \mathrm{~mL}$ of $1 \mathrm{M} \mathrm{HCl}$. Samples were 
subjected to ultrasonication for 5 minutes at 100\% amplitude (Branson Bransonic 32; SigmaAldrich Corp. St. Louis, MO, USA) then centrifuged for 15 minutes at $3000 \mathrm{rpm}$. The supernatant was removed for sampling and stored in brown glass vials in a refrigerator $\left(2-8{ }^{\circ} \mathrm{C}\right)$.

Stability studies were completed for the samples, varying time between sample extraction to injection on the ion chromatograph as well as storage conditions of temperature, light, and glass versus plastic storage containers. It was found that plastic Eppendorf tubes were not effective for long term sample storage, and neither was storage at room temperature. Under these circumstances, the oxidation state of iron was observed to change quickly over a short time $(<2$ h). Storing samples in brown glass vials placed in the refrigerator allowed sample stability (no change in iron oxidation states) for up to 6 hours.

Iron standards were made in $0.1 \mathrm{M} \mathrm{HCl}$. Ferric chloride $\left(\mathrm{FeCl}_{3}, 1 \mathrm{mg} \mathrm{mL}-1\right)$ was used as a ferric iron standard and ferrous sulfate hexahydrate $\left(\mathrm{FeSO}_{4} \cdot 6 \mathrm{H}_{2} \mathrm{O}, 1 \mathrm{mg} \mathrm{mL} \mathrm{L}^{-1}\right)$ was used as the ferrous iron standard. The ferric chloride sample was then diluted 50-fold for standard curve generation, while the ferrous sulfate was diluted 10 -fold. On average, it was observed that $3 \%$ of the ferric standard was reduced to ferrous iron when measured with ion chromatography chemiluminescence likely due to a solvent effect.

The analytical system consisted of a Thermo Scientific Dionex AXP Metal-Free HPLC with a Rheodyne metal-free injector and PEEK tubing $1 / 20 \mathrm{~cm}$ inner diameter. The ion chromatography column was a Thermo Scientific IonPac CS5A $(4 \times 50 \mathrm{~mm}$ guard column and 4 $\times 250 \mathrm{~mm}$ analytical column) designed to separate a broad range of metal complexes by cation and anion chromatography. The outlet of the column was connected to a mixing tee, where the post-column reagents were introduced with the column effluent to elicit the chemilumiescent reaction. Chemiluminescence was measured with the HERM data monitor (HERM LB 500, 
Berthold Technologies LLC, Oak Ridge, TN, USA) to allow quantification of iron in its oxidation states. Ferric iron had a retention time of approximately 4.3 minutes, while ferrous iron had a retention time of approximately 8.2 minutes.

In the analytical system, sterile water (HyPure ${ }^{\mathrm{TM}}$ WFI Quality Water, HyClone Laboratories, Logan, UT, USA) was used in solvent preparation to avoid introduction of additional iron to the samples. The column was rinsed with the WFI Quality Water, followed by flowing $1 \mathrm{M} \mathrm{Na}_{2} \mathrm{SO}_{3}$ at $0.5 \mathrm{~mL} \mathrm{~min}^{-1}$ for 45 minutes to remove any dissolved oxygen on the column. During this time, the pre-column eluent (10 mM 2,4-pyridinedicarboxylic acid [PDCA], $80 \mathrm{mM}$ potassium chloride, $37 \mathrm{mM}$ formic acid, and $33 \mathrm{mM}$ potassium hydroxide $\mathrm{pH}$ adjusted to 4) was bubbled with argon gas to remove dissolved oxygen. The pre- and post-column reagents were flowed through the system at rates of 1.00 and $0.75 \mathrm{~mL} \mathrm{~min}^{-1}$, respectively. The postcolumn reagents consisted of $600 \mathrm{mM}$ hydrogen peroxide and $0.025 \mathrm{mM}$ luminol with $50 \mathrm{Mm}$ sodium carbonate $\mathrm{pH}$ adjusted to $11.5^{79}$. Standard curves were generated daily prior to sample injection and samples were injected in triplicate.

Size-Exclusion Chromatography used in Seed Ferritin Analysis- A stainless steel HighPerformance Liquid Chromatography (HPLC) Sonntek Pump was connected to a Rheodyne injector with a $20 \mu \mathrm{L}$ PEEK injection loop to separate biological metabolites on an Agilent Zorbax GF-250 $4 \mu \mathrm{m}$ size exclusion column $(4.6 \times 250 \mathrm{~mm})$. Samples were quantified on an Azura UVD 2.15 Knauer UV detector set to $254 \mathrm{~nm}^{80}$. Mobile phase was pumped at $0.5 \mathrm{~mL}$ $\min ^{-1}$ and consisted of $0.2 \mathrm{M}$ ammonium sulfate with $0.05 \mathrm{M}$ tris- $\mathrm{HCl}$ buffer at $\mathrm{pH}$ of 7 . An antimicrobial was added $(0.005 \%$ sodium azide) to maintain column integrity throughout the study. Maize kernels were acquired from the courtyard grown maize harvest and analyzed for the amount of ferritin across treatment types previously described. Dried kernels $(\sim 0.5 \mathrm{~g})$ were 
pulverized between plastic sheets using a wooden mallet and placed in $1 \mathrm{~mL}$ of $50 \mathrm{mM}$ tris- $\mathrm{HCl}$ buffer $(\mathrm{pH} 7)$ and ultrasonicated for two minutes at $100 \%$ amplitude. The sample was then heat treated at $75{ }^{\circ} \mathrm{C}$ for 10 minutes followed by immediate cooling on ice for 15 minutes.

Centrifugation 15,000 g for 30 minutes pulled large particulates out of the supernatant which was injected $(20 \mu \mathrm{L})$ for quantification against a ferritin standard (MyBioSource, Inc., San Diego, CA, USA).

Principal Component Analysis of ${ }^{59} \mathrm{Fe}$ Data- The information included in our ${ }^{59} \mathrm{Fe}^{2+/ 3+}$ allocation measurements were represented by two principal components (PC), with PC1 representing $86.07 \%$ of the information embedded in the data and PC2 13.93\%. The PCs selected to represent the data are classified as feature vectors (F1 and F2) as shown on the biplot (Figure 3.3.1. E). The axes are in terms of the eigenvalues, with larger values indicating a greater variance, thus a greater representation of the information within the data. The active variables shown in gray represent the initial variables of allocation of $\mathrm{Fe}^{2+}$ and $\mathrm{Fe}^{3+}$ in maize. The length of the active variable vectors indicates how well the variables are tied to the feature vectors. Since both the active variable vectors are equivalent in length and are found equally between F1 and F2 it can be interpreted that both active variables are equally represented by both F1 and F2. Statistical Analysis- Data was subjected to the Shapiro-Wilk Normality Test to identify outliers so all data groups reflected normal distributions. Data was analyzed using the Student's t-test for pair-wise comparisons made between non-inoculated controls and treatment. Statistical significance was set at $\mathrm{P}<0.05$.

\subsection{Abbreviations}

$\begin{array}{ll}\text { PGPB } & \text { Plant growth promoting bacteria } \\ \text { A. brasilense } & \text { Azospirillum brasilense rhizobacteria } \\ \text { IAA } & \text { Carbon dioxide } \\ \text { DAG } & \text { Days after germination } \\ \text { SE } & \text { Standard Error }\end{array}$




$\begin{array}{ll}\text { rpm } & \text { Rotations per minute } \\ \text { CFU } & \text { Colony forming unit } \\ \text { TLC } & \text { Thin Layer Chromatography } \\ \text { UV/VIS } & \text { Ultraviolet Visible Spectroscopy } \\ \text { HCl } & \text { Hydrochloric acid } \\ \text { BNF } & \text { Biological nitrogen fixation }\end{array}$




\section{References}

1) Verhagen BW, Glazebrook J, Zhu T, Chang HS, van Loon LC, Pieterse CM. The transcriptome of rhizobacteria-induced systemic resistance in Arabidopsis. Mol. PlantMicrobe Inter. 2004; 17: 895-908.

2) Richardson A, Barea J-M, McNeill A,Prigent-Combaret C. Acquisition of phosphorus and nitrogen in the rhizosphere and plant growth promotion by microorganisms. Plant and Soil 2009; 321: 305-339.

3) Pankievicz VCS, Amaral FP, Santos KFD, Agtuca B, Xu Y, Schueller MJ, Arisi ACM, Steffens MBR, de Souza EM, Pedrosa FO, Ferrieri RA, Stacey G. Robust biological nitrogen fixation in a model grass-bacterial association. The Plant Journal 2015; 81: 907919.

4) Fibach-Paldi S, Burdman S, Okon Y. Key physiological properties contributing to rhizosphere adaptation and plant growth promotion abilities of Azospirillum brasilense. FEMS Microbiol. Lett. 2012; 326: 99e108.

5) Brusamarello-Santos LCC, Alberton D, Valdameri G, Camilios-Neto D, Covre R, Lopes K, Tadra-Sfeir MZ, Faoro H, Monteiro R, Silva AB, Broughton WJ, Pedrosa FO, Wassem R, de Souza EM. Modulation of defence and iron homeostasis genes in rice roots by the diazotrophic endophyte Herbaspirillum seropedicae. Scientific Reports 2018; 9:10573.

6) Devendra SD and Prasanna R. Potential of microbes in the biofortification of $\mathrm{Zn}$ and Fe in dietary food grains. A review. Agronomy for Sustainable Development 2020; 40: 1536.

7) Bashan Y, Holguin G, de-Bashan LE. Azospirillum-plant relationships: physiological, molecular, agricultural, and environmental advances. Canadian Journal of Microbiology 2004; 50: 521-577.

8) Dobbelaere S, Croonenborghs A, Thys A, Ptacek D, Vanderleyden J, Dutto P, Labandera-Gonzalez C, Caballero-Mellado J, Aguirre JF, Kapulnik Y. Responses of agronomically important crops to inoculation with Azospirillum. Functional Plant Biology 2001; 28: 871-879.

9) Hungria M, Campo R, de Souza E, Pedrosa F. Inoculation with selected strains of Azospirillum brasilense and A. lipoferum; improves yields of maize and wheat in Brazil. Plant and Soil 2010; 331: 413-425.

10) Scott S, Housh A, Powell G, Benoit M, Anstaett A, Gerheart A, Wilder SL, Schueller MJ, Ferrieri RA. Azospirillum brasilense (HM053) boosts corn yield and ferritin content. MDPI-Agronomy Journal 2020; 10: 394-405.

11) Zuo Y and Zhang F. Soil and crop management strategies to prevent iron deficiency in crops. Plant Soil 2011; 339: 83-95.

12) Samaranayake P, Peiris BD, Dssanayake S. Effect of excessive ferrous $(\mathrm{Fe}+2)$ on growth and iron content in rice (Oryza sativa) Int. J. Agri. Biol. 2012; 14: 296-298.

13) Mahender A, Swamy BPM Anandan A, Ali J. Tolerance of iron-deficient and - toxic soil conditions in rice. Plants 2019; 8: 31-35. 
14) Curie $\mathrm{C}$ and Briat JF. Iron transport and signaling in plants. Annu. Rev. Plant Biol. 2003; 54: 183-206.

15) Fageria NK, Baligar VC, Li YC. The role of nutrient efficient plants in improving crop yields in the twenty first century. J. Plant Nutri. 2008; 31: 1121-1157.

16) Römheld V and Marschner H. Mechanism of iron uptake in peanut plants I. Fe reduction, chelate splitting and release of phenolics. Plant Physiol. 1983; 71: 949-954.

17) Marschner H and Römheld V. Strategies of plant for acquisition of iron. Plant Soil 1994; 165: $261-274$.

18) Jeong $\mathrm{J}$ and Guerinot ML. Homing in on iron homeostasis in plants. Trends Plant Sci. 2009; 14: 280-285.

19) Cesco S, Neumann G, Tomasi N, Pinton R, Weisskopf L. Release of plant borne flavanoids into the rhizosphere and their role in plant nutrition. Plant Soil 2010; 329: 125.

20) Jeong $\mathrm{J}$ and Connolly EL. Iron uptake mechanisms in plants: Functions of FRO family of ferric reductases. Plant Sci. 2009; 176: 709-714.

21) Römheld V and Marschner H. Evidence for a specific uptake system for iron phytosiderophore in roots of grasses. Plant Physiol. 1986; 80: 175-180.

22) Römheld V. Different strategies for iron acquisition in higher plants. Plant Physiol. 1987; 70: 231-234.

23) Lin Y F, Liang HM, Yang SY, Boch A, Clemens S, Chen CC, Wu JF, Huang JL, Yeh $\mathrm{KC}$. Arabidopsis IRT3 is a zinc-regulated and plasma membrane localized zinc/iron transporter. New Phytol. 2009; 182: 392-404.

24) Li S, Zhou X, Huang Y, Zhu L, Zhang S, Zhao Y, Guo J, Chen J, Chen R. Identification and characterization of the zinc-regulated transporters, iron-regulated transporter-like protein (ZIP) gene family in maize. BMC Plant Biology 2013; 13: 114-128.

25) Ishimaru Y, Suzuki M, Tsukamoto T, Suzuki K, Nakazono M, Kobayashi T. Rice plants take up iron as an $\mathrm{Fe}^{+3}$-phytosiderophre and as Fe. Plant J. 2006; 45: 335-346.

26) Curie C, Panaviene Z, Loulergue C, Dellporta SL, Briat JF, Walker EL. Maize yellow stripel encodes a membrane protein directly involved in Fe(III) uptake. Nature 2001; 409: 346-349.

27) Bao Z, Bai J, Cui H, Gong C. A missing link in radial ion transport: Ion transporters in the endodermis. Front. Plant Sci. 2019; 10, Article 713: 1-8.

28) Barberon M. The endodermis as a checkpoint for nutrients. New Phytol. 2017; 213: 1604-1610.

29) Von Wiren N, Klair S, Bansal S, Briat JF, Khodr H, Shioiri T, Leigh RA, Hider RC. Nicotianamine chelates both Fe(III) and Fe(II). Implications for metal transport in plants. Plant Physiol. 1999; 119: 1107-1114.

30) Durrett TP, Gassmann W, Rogers EE. The FRD3-mediated efflux of citrate into the root vasculature is necessary for efficient iron translocation. Plant Physiol. 2007; 144: 197205. 
31) Rellán-Álvarez R, Abadía J, Álvarez-Fernández, A. Formation of metal-nicotianamine complexes as affected by $\mathrm{pH}$, ligand exchange with citrate and metal exchange. A study by electrospray ionization time-of-flight mass spectrometry. Rapid Commun. Mass Sp. 2008; 22: 1553-1562.

32) Rellán-Álvarez R, Giner Martínez Sierra J, Orduna J, Orera I, Rodríguez Castrillón, JÁ, García-Alonso JI, Abadía J, Álvarez-Fernández A. Identification of a tri-iron(III), tricitrate complex in the xylem sap of iron-deficient tomato resupplied with iron: New insights into plant iron long-distance transport. Plant Cell Physiol. 2009; 51: 91-102.

33) Inoue H, Higuchi K, Takahashi M, Nakanishi H, Mori S, Nishizawa NK. Three rice nicotianamine synthase genes, OsNAS1, OsNAS2, and OsNAS3 are expressed in cells involved in long-distance transport of iron and differentially regulated by iron. Plant $J$. 2003; 36: 366-381.

34) Bonneau J, Baumann U, Beasley J, Li Y, Johnson AAT. Identification and molecular characterization of the nicotianamine synthase gene family in bread wheat. Plant Biotechnol. J. 2016; 14: 2228-2239.

35) Kerkeb L and Krämer U. The role of free histidine in xylem loading of nickel in Alyssum lesbiacum and Brassica juncea. Plant Physiol. 2003; 131: 716-724.

36) Ravanbakhsh M, Kowalchuk GA, Jousset A. Root-associated microorganisms reprogram plant life history along the growth-stress resistance trade-off, The ISME Journal 2019; 13: 3093-3101.

37) Machado HB, Funayama S, Rigo LU, Pedrosa FO. Excretion of ammonium by Azospirillum brasilense mutants resistant to ethylenediamine. Can. J. Microbiol. 1991; 37: 549e553.

38) Schenberger Santos AR, Etto RM, Furmam RW, de Freitas DL, d'Eça Nogueira Santos KF, de Souza EM, Pedrosa FO, Ayub RA, Steffens MBR, Galvão CW. Labeled Azospirillum brasilense wild type and excretion-ammonium strains in association with barley roots. Plant Physiology and Biochemistry 2017; 118: $422 \mathrm{e} 426$.

39) Pedrosa FO and Yates MG. Regulation of nitrogen fixation (nif) genes of Azospirillum brasilense by nifA and ntr (gln) type gene products (Azospirillum brasilense; Nif mutants; nifA; ntrC; MoFe protein). FEMS Microbiology Letters 1984; 23: 95-101.

40) Näsvall J, Knöppel A, Andersson DI. Duplication-Insertion Recombineering: a fast and scar-free method for efficient transfer of multiple mutations in bacteria. Nucleic Acids Res. 2017; 45(5):e33.

41) de Souza EM., private communication.

42) Ferrieri RA and Wolf AP. The chemistry of positron-emitting nucleogenic (hot) atoms with regard to preparation of labeled compounds of practical utility. Radiochim. Acta. 1983; 34: 69-83.

43) Ferrieri RA. "Production and application of synthetic precursors labeled with carbon-11 and fluorine-18" in Handbook of Radiopharmaceuticals: Radiochemistry and Applications. (Welch M.J. and Redvanly C.S., eds.) John Wiley \& Sons, Ltd., Chichester, England (2003).

44) Ferrieri RA, Gray DW, Babst BA, Schueller MJ, Schlyer DJ, Thorpe MR, Orians CM, Lerdau M. Use of carbon-11 in Populus shows that exogenous jasmonic acid increases biosynthesis of isoprene from recently fixed carbon. Plant, Cell and Environment, 2005; 25: 591-602. 
45) Qu W, Robert CAM, Erb M, Hibbard BE, Paven M, Gleede T, Riehl B, Kersting L, Cankaya AS, Kunert AT, Xu Y, Schueller MJ, Shea C, Alexoff D, Lee SJ, Fowler JS, Ferrieri RA. Dynamic precision phenotyping reveals mechanisms of crop tolerance to root herbivory. Plant Physiology 2016; 172: 776-788.

46) Klaus R, Fisher W, Hauck HE. Application of a thermal in situ reaction for fluorometric detection of carbhhydrates on $\mathrm{NH}_{2}$-layers. Chromatographia 1990; 29: 467-472.

47) Babst BA, Karve A, Judt T. Radio-metabolite analysis of carbon-11 biochemical partitioning to nonstructural carbohydrates for integrated metabolism and transport studies. Plant Cell Physiol. 2013; 54: 1016-1025.

48) Lee SJ, Fowler JS, Alexoff DL, Schueller MJ, Kim D, Nauth AA, Weber C, Kim SW, Hooker JM, Mad L, Qu W. An efficient and practical synthesis of $\left[2-{ }^{11} \mathrm{C}\right]$ indole via superfast nucleophilic $\left[{ }^{11} \mathrm{C}\right]$ cyanation and RANEY ${ }^{\circledR}$ Nickel catalyzed reductive cyclization. Org. Biomol. Chem. 2015; 13: 11235-11243.

49) Onidasa D, Stachnik JM, Brucker S, Kratzig S, Gerwert K. Histidine is involved in coupling proton uptake to electron transfer in photosynthetic proteins. European J. Cell Biology 2010; 89: 983-989.

50) Ingle RA. "Histidine Biosynthesis" in The Arabidopsis Book, a publication from the American Society of Plant Biologists, 2011; doi: 10.1199/tab.0141.

51) Connorton JM, Balk J, Rodríguez-Celma J. Iron homeostasis in plants - a brief overview. Metallomics 2017; 9: 813-823.

52) Frey M, Spiteller D, Boland W, Gierl A. Transcriptional activation of Igl, the gene for indole formation in Zea mays: a structure-activity study with elicitor-active $\mathrm{N}$-acyl glutamines from insects. Phytochemistry 2004; 65: 1047-1055.

53) Frey M, Chomet P, Glawischnig E, Stettner C, Grün S, Winklmair A, Eisenreich W, Bacher A, Meeley RB, Briggs SP, Simcox K, Gierl A. Analysis of a chemical plant defense mechanism in grasses. Science 1997; 277: 696-699.

54) Frey M, Schullehner K, Dick R, Fiesselmann A, Gierl A. Benzoxazinoid biosynthesis, a model for evolution of secondary metabolic pathways in plants. Phytochemistry 2009; 70: 1645-1651.

55) Zhou S, Richter A, Jander G. Beyond defense: multiple functions of benzoxazinoids in maize metabolism. Plant Cell Physiol. 2018; 59: 1528-1537.

56) Hu L, Mateo P, Ye M, Zhang X, Berset JD, Handrick V, Radisch D, Grabe V, Kollner TG, Gershenzon J, Robert CAM, Erb M. Plant iron acquisition strategy exploited by an insect herbivore. Science 2018; 361: 694-697.

57) Frey M, Stettner C, Paré PW, Schmelz EA, Tumlinson JH, Gierl A. An herbivore elicitor activates the gene for indole emission in maize. Proc. Nat'l Acad Sci. U.S.A. 2000; 97: 14801-14806.

58) Erb M, Veyrat N, Robert CAM, Xu H, Frey M, Ton J, Turlings TCJ. Indole is an essential herbivore-induced volatile priming signal in maize. Nature Communications 2015; 16: 6273-6283.

59) Band LR, Wells DM, Larrieu A, Sun J, Middleton AM, French AP, Brunoud G, Sato EM, Wilson MH, Péret B, Oliva M, Swarup R, Sairanen I, Parry G, Ljung K, Beeckman T, Garibaldi JM, Estelle M, Owen MR, Vissenberg K, Hodgman TC, Pridmore TP, King JR, Vernoux T, Bennett MJ. Root gravitropism is regulated by a transient lateral auxin 
gradient controlled by a tipping-point mechanism. Proc. Nat. Acad. Sci. U.S.A. 2012; 109: 4668-4673.

60) Madhaiyan M, Poonguzhali S, Sa T. Characterization of 1-aminocyclopropane-1carboxylate (ACC) deaminase containing Methylobacterium oryzae and interactions with auxins and ACC regulation of ethylene in canola (Brassica campestris) Planta 2007; 226: $867-876$.

61) Zhang Z, Barlow JN, Baldwin JE, Schofield CJ. Metal-catalyzed oxidation and mutagenesis studies on the iron(II) binding site of 1-aminocyclopropane-1-carboxylate oxidase Biochemistry 1997; 36: 15999-16007.

62) Alberton D, Müller-Santos M, Brusamarello-Santos LCC, Valdameri G, Cordeiro FA, Yates MG, Pedrosa FO, de Souza EM. Comparative proteomics analysis of the rice roots colonized by Herbaspirillum seropedicae strain SmR1 reveals induction of the methionine recycling in the plant host. J. Proteome Res. 2013; 12: 4757-4768.

63) Glick BR. Modulation of plant ethylene levels by the bacterial enzyme ACC deaminase. FEMS Microbiology Letters, 2005; 251: 1-7.

64) Thao NP, Khan MIR, Thu NBA, Hoang XLT, Asgher M, Khan NA, Tran LS. Role of ethylene and its cross talk with other signaling molecules in plant responses to heavy metal stress. Plant Physiol. 2015; 169: 73-84.

65) Keunen E, Schellingen K, Vangronsveld J,Cuypers A. Ethylene and metal stress: small molecule, big impact. Front Plant Sci. 2016; 7: 23.

66) Kende H. Ethylene biosynthesis. Annu Rev Plant Physiol Plant Mol Biol 1993; 44: 283307.

67) Holguin G, Glick BR. Expression of the ACC deaminase gene from Enterobacter cloacae UW4 in Azospirillum brasilense. Microb Ecol 2001; 41: 281-288.

68) Benková E and Hejátko J. Hormone interactions at the root apical meristem. Plant Mol. Biol. 2009; 69: 383-396.

69) Muday GK, Rahman A, Binder BM. Auxin and ethylene: collaborators or competitors? Trends Plant Sci. 2012; 17: 181-195.

70) Van de Poel B, Smet D, Van Der Straeten D. Ethylene and hormonal crosstalk in vegetative growth and development. Plant Physiol. 2015; 169: 61-72.

71) $\mathrm{Hu} Y$, Vandenbussche F, Van Der Straeten D. Regulation of seedling growth by ethylene and the ethylene-auxin crosstalk. Planta 2017; 245: 467-489.

72) Ruzicka K, Ljung K, Vanneste S, Podhorská R, Beeckman T, Friml J, Benková E. Ethylene regulates root growth through effects on auxin biosynthesis and transportdependent auxin distribution. Plant Cell 2007; 19: 2197-2212.

73) Stepanova AN, Yun J, Likhacheva AV, Alonso JM. Multilevel interactions between ethylene and auxin in Arabidopsis roots. Plant Cell 2007; 19: 2169-2185.

74) Swarup R, Perry P, Hangenbeek D, Van Der Straeten D, Beemster GT, Sandberg G. Bhalerao R, Ljung K, Bennett MJ. Ethylene upregulates auxin biosynthesis in Arabidopsis seedlings to enhance inhibition of root cell elongation. Plant Cell 2007; 19: 2186-2196.

75) Neal AL, Ahmad S, Gordon-Weeks R, Ton J. Benzoxazinoids in Root Exudates of Maize Attract Pseudomonas putida to the Rhizosphere. PLoS ONE 2012; 7(4): e35498. 
76) Waller S, Wilder SL, Schueller MJ, Housh AB, Ferrieri RA. Quantifying plant-borne carbon assimilation by root-associating bacteria. Microorganisms 2020; 8: 700-710.

77) Morella, N.M.; Weng, F.C.-H.; Joubert, P.M.; Metcalf, C.J.E.; S. Lindow, S.; Koskella, B. Successive passaging of a plant-associated microbiome reveals robust habitat and host genotype-dependent selection. 2019, bioRxiv doi:10.1101/627794

78) Briat, J.-F.; Ravet, K.; Arnaud, N.; Duc C.; Boucherez J.; Touraine, B.; Cellier F.; Gaymard, F. New insights into ferritin synthesis and function highlight a link between iron homeostasis and oxidative stress in plants. Ann. Bot. 2010, 105, 811-822.

79) Chen, Y.C.; Jian, Y.L.; Chiu K.H.; Yak H.K. Simultaneous speciation of iron(II) and iron(III) by ion chromatography with chemiluminescence detection. Anal Sci. 2012, 28, 795-799

80) Takatera, K.; T. Watanabe, T. High-performance liquid chromatographic determination of iron-containing proteins with on-line inductively coupled plasma mass spectrometric detection. Anal. Sci. 1991, 7, 695-698. 


\section{Chapter 4: Plant Growth Promoting Bacteria Modulate Maize Zinc Assimilation: An}

\section{Examination of the Mechanisms of Action using Functional Mutants of Azospirillum}

\section{brasilense}

\subsection{Preface}

This project is not currently submitted for publication, but is accepted to Multidisciplinary Digital Publishing Institute (MDPI) Agronomy conference for oral presentation in May 2021, with the possibility of subsequent invitation to publish with the MDPI publishing house in their Agronomy journal. I will be first author on the manuscript and I was involved in the administration of the radiotracer $\left({ }^{65} \mathrm{Zn}\right)$ to live plants and subsequent autoradiography imaging, NaI radioactivity in the separate plant tissues and for physiology measurements, measurement of $\mathrm{Zn}$ in the plant tissues via ICP-MS and LA-ICP-MS, PCA analysis, and method development of the Zn quantification in seeds via ion chromatography. Data collection for Zn content of seed was contributed by Mary Benoit of the U. of Missouri Division of Plant Sciences.

\subsection{Introduction}

Zinc $(\mathrm{Zn})$ has been implicated with a broad spectrum of growth characteristics in higher plants. For example, in apple, visible symptoms of Zn deficiency occur in dicotyledons where significant decrease in leaf size is $\operatorname{seen}^{1}$ (p. 559). This trait, coined "little leaf" syndrome, is observed as a common growth characteristic in many fruit tree species subjected to $\mathrm{Zn}$ deficiency ${ }^{2}$. Zn also plays important roles in many biochemical functions within plants. It is an essential component of over 300 enzymes ${ }^{3}$. It also plays a role in DNA and RNA metabolism, cell division, and protein synthesis ${ }^{4}$. A lack of sufficient Zn during plant growth can decrease yield and crop quality because of the disruption in these normal metabolic functions ${ }^{5-7}$. 
Today, approximately $30 \%$ of global crop production is lost due to essential nutrient deficiencies caused by climatic extremes that result in excessive soil weathering ${ }^{8}$ and by a lack of diverse agricultural practices that deplete nutrient levels in soil. Additionally, foods produced from $\mathrm{Zn}$-deficient crops can result in human $\mathrm{Zn}$ deficiency, which can in turn have an impact on human well-being by reducing the immune functions and increasing the risk of growth stunting in children or the risk of adverse pregnancy outcomes in women ${ }^{5,9}$.

Plant growth promoting bacteria (PGPB), which can help their host weather difficult conditions and potentially increase nutritional value of crops, are finding increased use in agriculture. These organisms can activate physiological and biochemical responses within their host for mutual benefit to build natural tolerances to environmental stresses and thereby reduce losses in the field ${ }^{10-16}$. Several have been identified as endophytes of grass species, including Azoarcus spp. in Kallar grass (Leptochloa fusca (L.) Kunth) and rice (Oryza sativa) ${ }^{17-19}$, Herbaspirillum seropedicae in sugarcane (Saccharum officinarum) ${ }^{20}$ and sorghum (Sorghum bicolor) ${ }^{19}$, and Gluconacetobacter diazotrophicus in sugarcane ${ }^{21}$. Others have been identified as epiphytes, including Azospirillum brasilense and Azospirillum lipoferum, which have been commercialized as crop inoculants for maize and wheat ${ }^{22-25}$. These strains are gaining increasing acceptance in agriculture as PGPB inoculants. Unlike rhizobia that form an intracellular symbiosis with their legume hosts, PGPB do not induce the formation of observable plant structures (nodules). They are also usually not major components of the soil microflora ${ }^{20,26}$. These $\mathrm{N}_{2}$-fixing bacteria infect at the emergence of lateral roots and in the zone of elongation and differentiation above the root tip ${ }^{14}$. Typically, very high numbers of PGPB in roots have been reported (i.e., $\leq 10^{8} /$ gram root dry weight) with no observable disease symptoms ${ }^{17}$. 
The present work reports on the use of radioactive $\mathrm{Zn}-65$ ( $\mathrm{t} / \mathrm{2} 244$ days) to examine root assimilation and whole-plant transport of the metal under different conditions of growth. A review of the literature reveals a limited number of papers that have used $\mathrm{Zn}$ radioisotopes to examine plant uptake of the metal ${ }^{27-29}$. Measuring $\mathrm{Zn}$ uptake through its radioactive decay can be highly quantitative. However, its general utility in plant biology is limited by the fact that many laboratories are not equipped with the appropriate nuclear instrumentation needed to make such measurements. Here, we inoculated plants with three different functional mutant strains of Azospirillum brasilense PGPB including HM053, a $\mathrm{Nif}^{+}$constitutively expressed mutant of the nif gene coding for nitrogen fixation enzymes that fixes excess $\mathrm{N}_{2}$ and excretes large amounts of ammonium to the rhizosphere; ipdC, a mutant strain disrupted in the ipdC gene thus impaired in biosynthesizing the plant relevant hormone auxin (indole-3-acetic acid) and FP10, a Nif ${ }^{-}$mutant that is deficient in fixing $\mathrm{N}_{2}$ and compared plant performance for assimilating $\mathrm{Zn}-65$ relative to non-inoculated controls. Maize were grown outdoors to fruition and the resulting seeds were analyzed for $\mathrm{Zn}$ content. These studies were conducted in order to determine whether these microbial functions had any influence on their host's performance.

\subsection{Results and Discussion}

Results in Figure 4.3.1. showing different rates for Zn-65 transport as a function of $A$. brasilense inoculation showed that ipdC $>\mathrm{HM} 053>$ FP10. FP10 was most like non-inoculated control plants. Tissue distribution of $\mathrm{Zn}$ using 'cut and count' techniques revealed a similar dissimilarity between $i p d C$ bacteria and the other inoculants (Figure 4.3.2.). Systematic trends defining uptake and in planta translocation of $\mathrm{Zn}-65$ become apparent in the PCA biplot (Figure 4.3.3. A). The information included in the biplot was represented by two principal 

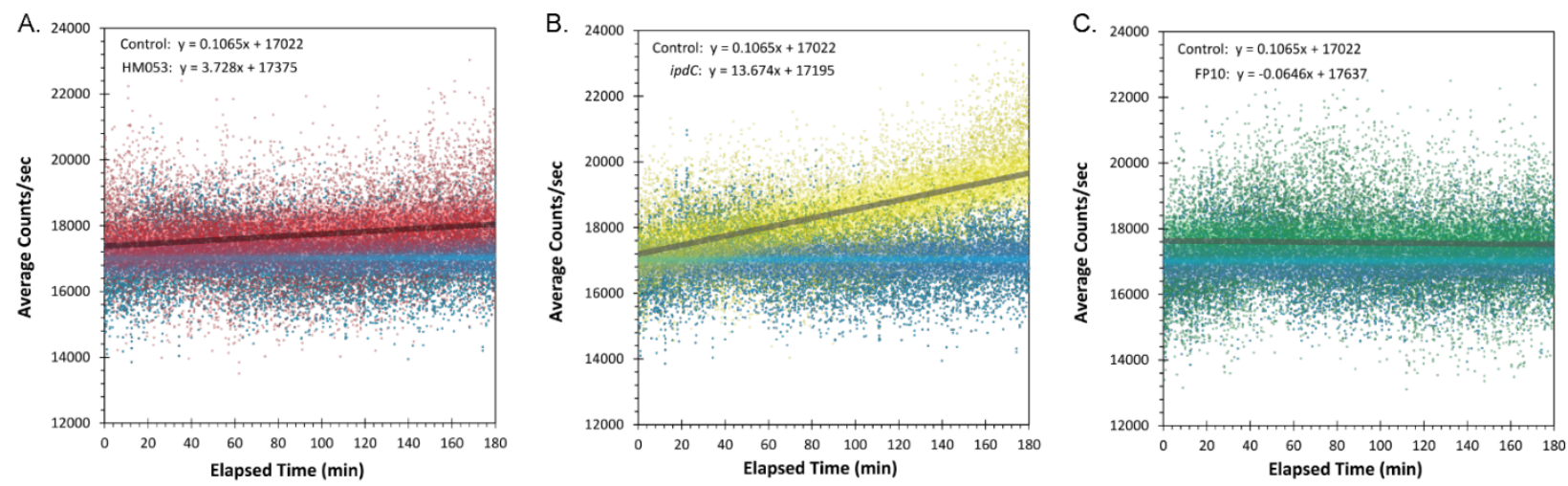

Figure 4.3.1. Dynamic Zn-65 transport over 3-hours of acquisition. Each data point reflects an average of $\mathrm{N}=5-6$ biological replicates sampled at $1 \mathrm{~Hz}$.

components (PC), with $\mathrm{PC} 1$ representing $71.89 \%$ of the information embedded in the data and

PC2 and 28.11\%. The PCs selected to represent the data are classified as feature vectors (F1 and F2) as shown on the biplot. The axes are in terms of the eigenvalues, with larger values indicating a greater variance, thus a greater representation of the information within the data. The active variables, shown as dotted lines, represent the initial variables of root assimilation of $\mathrm{Zn}$ 65 and shoot allocation. The length of the active variable vectors indicates how well the variables are tied to the feature vectors. Since both the active variable vectors are equivalent in length in Figure 4.3.3. A and are found equally between F1 and F2 it can be interpreted that both
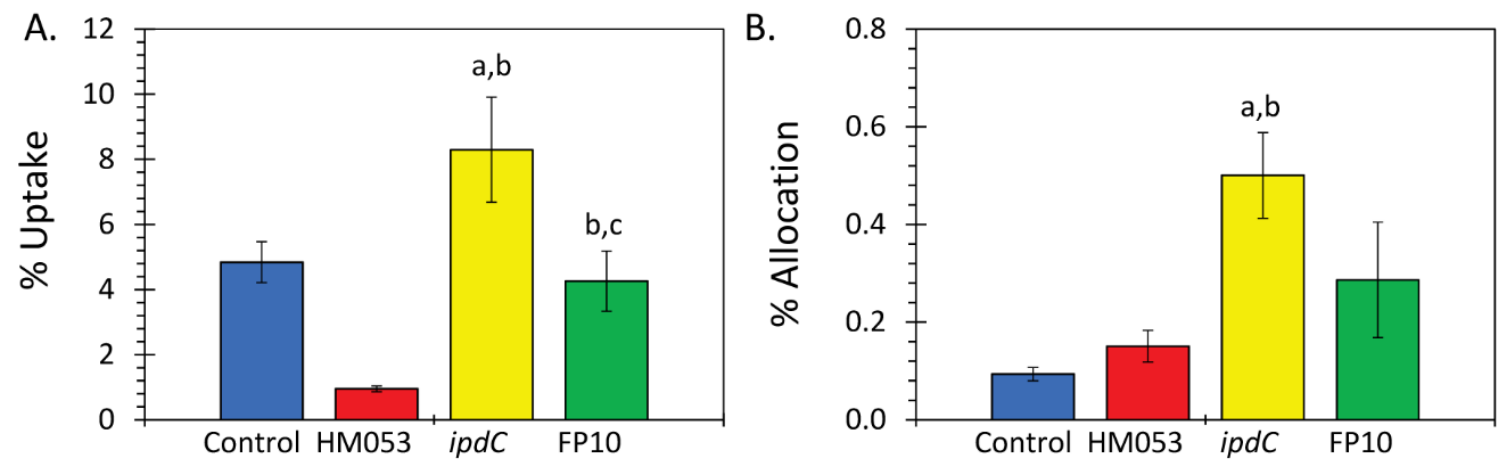

Figure 4.3.2. $\mathrm{NaI}(\mathrm{Tl})$ gamma detection of plant tissues exhibit differences in uptake and allocation of $\mathrm{Zn}-65$ associated with different functional mutants of $\boldsymbol{A}$. brasilense (Panel A); 'Cut and count' measurements yielded information on plant uptake of $\mathrm{Zn}-65$ presented as the percent of the $\mathrm{Zn}-65$ dose administered to the beaker that was assimilated by the plant over 3-hours. (Panel B); root-to-shoot allocation of $\mathrm{Zn}-65$ is presented as the percent of the administered dose of radiotracer. Data reflects means for $\mathrm{N}=5-6$ replicates $( \pm \mathrm{SE})$. Statistical significance $\mathrm{P}<0.05$ was designated by ' $\mathrm{a}$ ' in a comparison of treatment to control, ' $\mathrm{b}$ ' in a comparison of ipdC or FP10 to HM053 and 'c' in a comparison of FP10 to ipdC treatment. 

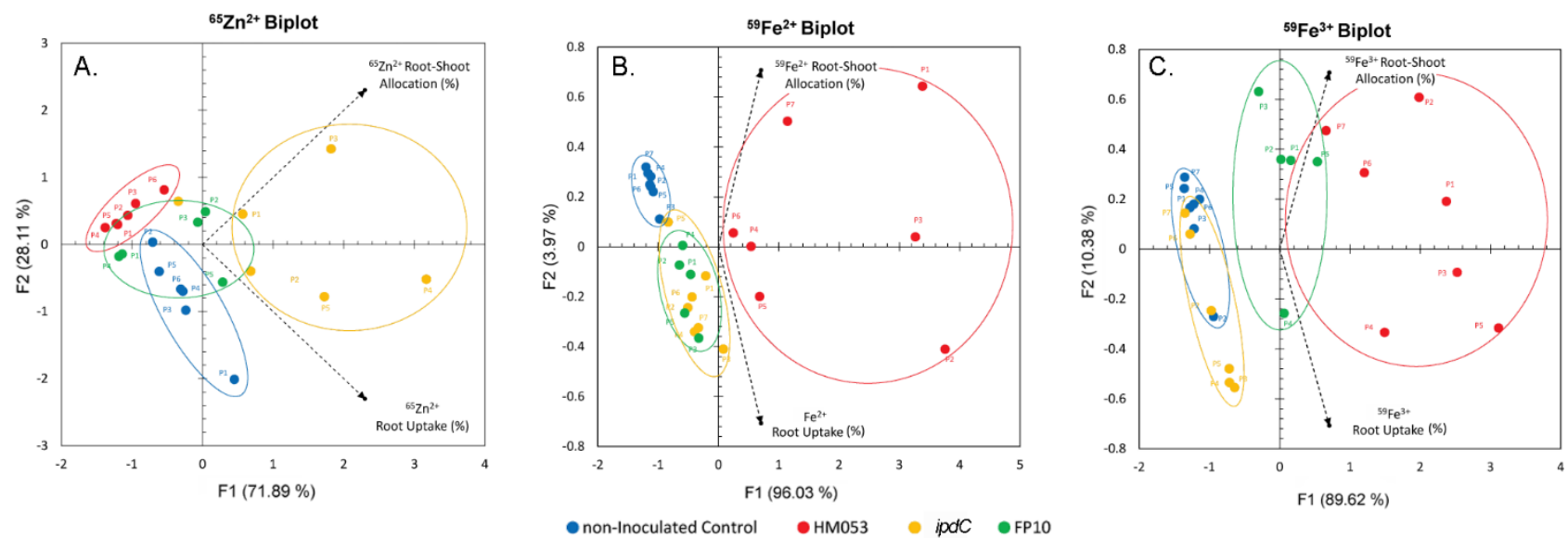

Figure 4.3.3. Principal component analysis correlates $\mathrm{Zn}-65$ uptake and root-shoot allocation (Panel A) to the biological functions of the beneficial microbes. HM053 and FP10 mutant strains were most like non-inoculated plant behavior while ipdC was dissimilar, exhibiting highest levels of root uptake and transport. Ellipses were added for visual aid and do not represent any significance in the data shown.

active variables are equally represented by both F1 and F2. As displayed, each of the microbial treatments clustered together, indicating behavior within a treatment-type that was distinct from other treatments. It showed that FP10 and non-inoculated maize were similar in overall Zn-65 uptake and shoot allocation behavior. HM053 inoculated maize exhibited a slight elevation in allocation patterns relative to control and FP10. ipdC was most unique in its uptake and allocation patterns than other treatments in the $\mathrm{X}$ - and $\mathrm{Y}$-axis direction.

What distinguishes ipdC from the other microbial inoculants examined in this study is its deficiency in producing auxin (indole-3-acetic acid), an important plant hormone. Our past studies showed that the HM053 mutant exhibited the highest level of auxin biosynthesis, being 2-times that of FP10 and 13-times that of $i p d C^{16}$. Furthermore, auxin biosynthesis in plants and $\mathrm{Zn}$ levels were strongly correlated. With tryptophan being the principal intermediate in auxin biosynthesis, withholding $\mathrm{Zn}$ was shown to lower plant tryptophan levels ${ }^{30}$, and auxin levels ${ }^{31}$, while exogenous treatment with $\mathrm{Zn}$ increased tryptophan levels ${ }^{32}$. We suspect that the mechanism for promoting plant $\mathrm{Zn}-65$ uptake in the present study has to do with the auxin producing capacity of the microorganism. We note that while ipdC lacks the ability to 
biosynthesize auxin, it still processes the molecular machinery to produce indole - a key precursor to tryptophan biosynthesis ${ }^{16}$. In fact, maize root indole emissions with ipdC inoculation were nearly 2-times that of HM053 inoculated plants, and 1.5-times that of FP10 inoculated plants ${ }^{33}$. We suspect this behavior may be due to bacteria-root indole trafficking which could elevate the endogenous pool of plant tryptophan causing an elevation in $\mathrm{Zn}$ uptake. To the best of our knowledge, no one has examined whether tryptophan treatments will elevate endogenous levels of plant $\mathrm{Zn}$.

Similar statistical treatments were applied to our previously published Fe-59 data ${ }^{16}$ both for ferrous (Fe (II)) and ferric (Fe (III)) forms of the tracer to yield Figures 4.3.3. B and C, respectively. As displayed here, each of the microbial treatments again clustered together, indicating behavior within a treatment-type that was distinct from the other microbial treatments. Unlike our Zn-65 data shown in Figure 4.3.3. A, we observed different microbial influences on host iron assimilation with HM053 exhibiting the greatest influence for root uptake and shoot allocation of both $\mathrm{Fe}$ (II) and $\mathrm{Fe}$ (III) over the other bacteria strains. In our earlier work, we ascertained through whole-plant radiographic imaging that the oxidation state of the iron radiotracer was unaltered by the microorganism's functions. Here we noted that each oxidation state of the radiometal exhibited a different spatial patterning across the shoot tissues with ${ }^{59} \mathrm{Fe}(\mathrm{III})$ accumulating in leaf tips while ${ }^{59} \mathrm{Fe}$ (II) accumulating uniformly throughout the leaves. Figure 4.3.4. shows an example of this distribution from HM053 inoculated maize plants since HM053 caused the largest increase in ${ }^{59} \mathrm{Fe}(\mathrm{III})$ and ${ }^{59} \mathrm{Fe}(\mathrm{II})$ allocations to shoots relative to the other microbial inoculants. For comparison, we also show radiographic images in the same figure from maize Zn-65 studies as a function of ipdC, FP10 and HM053 microbial inoculants. 


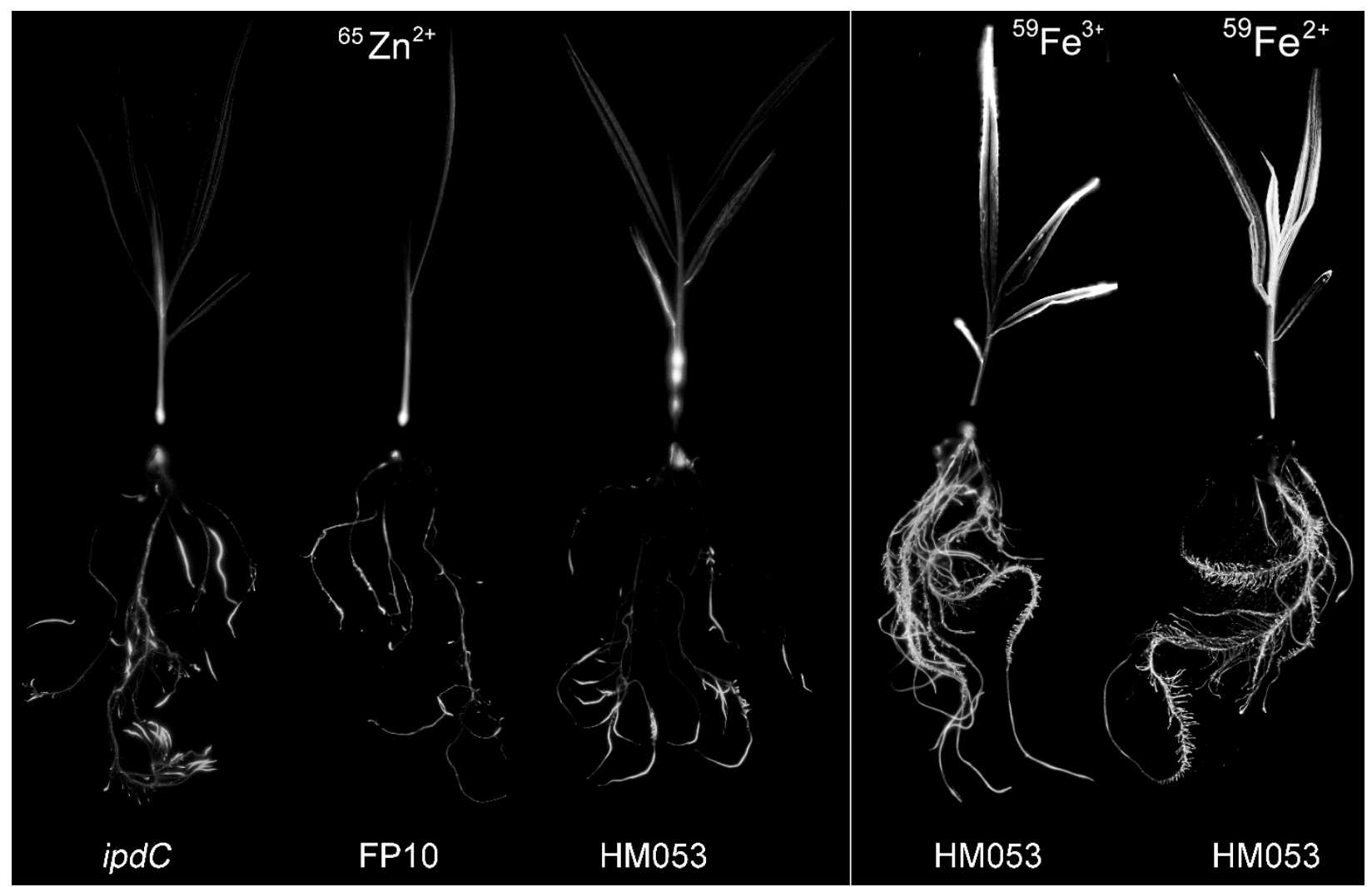

Figure 4.3.4. Radiographic images of maize plants after exposure to $\mathrm{Zn}-65$ as a function of inoculation with ipdC, FP10 and HM053 bacteria. For comparison, we also show radiographic images of maize plants after exposure to ${ }^{59} \mathrm{Fe}$ (III) and ${ }^{59} \mathrm{Fe}$ (II) tracers from our prior work ${ }^{15}$. These plants were also inoculated with HM053 which was shown to exert the greatest influence on host ${ }^{59} \mathrm{Fe}(\mathrm{III})$ and ${ }^{59} \mathrm{Fe}(\mathrm{II})$ uptake and allocation.

In all cases here, Zn-65 spatial patterning in leaves was like ${ }^{59} \mathrm{Fe}(\mathrm{II})$. However, root tissues

exhibited significantly different radiotracer distributions where elongation zones showed higher levels of $\mathrm{Zn}-65$ than both oxidation states of the Fe-59 radiotracer. Additionally, we noted a common trend where a high accumulation of $\mathrm{Zn}-65$ was observed in the lower stem region, likely in the coleoptile. Past studies have shown that the coleoptile in maize seedlings exhibits a strong growth dependency on auxin ${ }^{34}$. Taken together, our results show that maize assimilation of divalent metals such as $\mathrm{Zn}$ or Fe(II) have very different dependencies on microbial auxin biosynthesis. Once assimilated, these metals also exhibit very different spatial patterning during transport aboveground. 
Finally, we examined the longer-term influence of these mutant strains of $A$. brasilense on kernel $\mathrm{Zn}$ content. Results in Figure 4.3.5. showed that HM053 did not alter seed $\mathrm{Zn}$ levels relative to non-inoculated controls. However, both ipdC and FP10 bacteria showed significantly less $\mathrm{Zn}$ content. Hence, while ipdC

promotes $\mathrm{Zn}$ accumulation in host vegetative tissues, that action does not translate to the seed filling process. We suspect that heavy Zn-65 accumulation in the lower stem may minimize the metal's availability during seed filling.

\subsection{Conclusion}

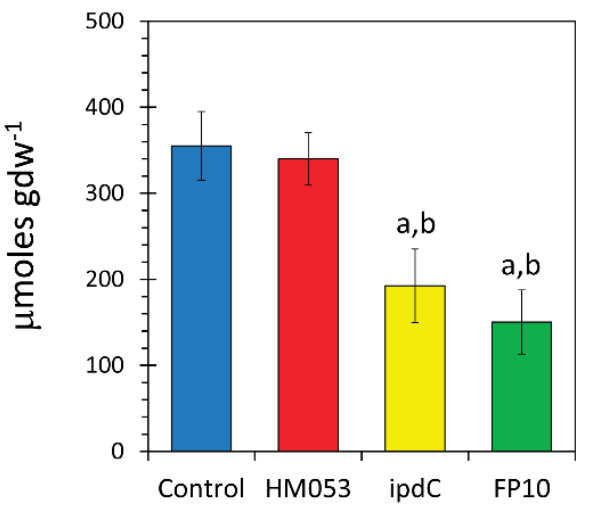

Figure 4.3.5. Kernel Zn content measured using ion chromatography. Data reflects means for $\mathrm{N}=12$ replicates $( \pm \mathrm{SE})$. Statistical significance $\mathrm{P}<0.05$ was designated by ' $a$ ' in a comparison of treatment to control, ' $\mathrm{b}$ ' in a comparison of ipdC or FP10 to HM053.

The present work showed evidence that certain biological traits of root-associating microorganisms can have beneficial effects on the host plant in promoting $\mathrm{Zn}$ uptake. These mechanisms of action appeared to correlate with the auxin producing capacity of the microorganism in that the auxin deficient mutant, ipdC had the greatest influence in promoting host Zn-65 uptake. Mechanisms of action appear not to be universally translated across all metals since Zn-65 and ${ }^{59} \mathrm{Fe}(\mathrm{II})$ exhibited very different dependencies on microbial functions.

While the microbial actions promoting host $\mathrm{Zn}$ uptake could benefit over the long term in improved crop yield, our results suggest that there is little or no effect on kernel zinc content improving its nutritional value.

\subsection{Materials and Methods}

Laboratory Plant Growth: Maize kernels from Elk Mound Seed Co. (Hybrid 8100) were dark germinated at room temperature for two days on sterilized paper towels wetted with sterile water in a petri-dish. Seeds were inoculated with bacteria culture as appropriate and transplanted to 
plastic seed germination pouches (Phytotc, Inc.) wetted with sterile Hoagland's basal salt solution for approximately one week. They were then transferred to individual $600 \mathrm{~mL}$ hydroponics cells that were continuously aerated and filled with Hoagland's nutrient (pH 6.0). Nutrient was exchanged on a five-day cycle. Growth conditions consisted of 12-hour photoperiods, $500 \mu \mathrm{mol} \mathrm{m} \mathrm{m}^{-2}$ light intensity, and temperatures of $25^{\circ} \mathrm{C} / 20^{\circ} \mathrm{C}$ (light/dark) with humidity at $70-80 \%$.

Outdoor Potted Plant Growth: For outdoor, non-radioactive studies, 3 maize kernels from Elk Mound Seed Co. (Hybrid 8100) were sown into each of 2.7-gallon pots filled with ProMix. Plants were placed on elevated tables outside and pots were rotated every week to ensure uniformity of growth conditions. After germination, any excess seedlings were removed from each pot leaving a single plant. A capful of fertilizer $(\sim 1.2 \mathrm{~g})$ containing nitrogen, phosphate and potash (14-14-14, Osmocote ${ }^{\mathrm{TM}}$ Smart-Release Plant Food Flower \& Vegetable ${ }^{\mathrm{TM}}$, The Scotts Company, Marysville, $\mathrm{OH}$ ) was added to the assigned pots at the time of planting. Fertilizer was reapplied to pots 30 and 60 days after germination (DAG). Study regimes included the following: (i) non-inoculated control plants; (ii) plants inoculated with A. brasilense HM053 bacterium; (iii) plants inoculated with $A$. brasilense ipdC bacterium; and (iv) plants inoculated with A. brasilense FP10 bacterium. Plants were administered liquid inoculants at 21, 42 and 63 DAG. Treatments were randomized across the planting platforms. At the end of the growing season, cobs were harvested, and seeds analyzed by ion chromatography for Zn content.

Bacteria Growth: Functional mutants were grown in liquid NFbHP-lactate medium following published procedures ${ }^{13}$. Cultures were washed with sterile water and diluted to approximately 1 $\mathrm{mL}$ containing $10^{6}$ to $10^{8}$ colony forming units per milliliter $\left(\mathrm{CFU} \mathrm{mL} \mathrm{m}^{-1}\right)$. Root inoculation involved adding the inoculum to a petri dish of 10-20 maize seedlings and rocking in a shaking 
incubator for two hours @ $30^{\circ} \mathrm{C}$. Seedlings were then placed into germination pouches for five days before transplanting to hydroponics.

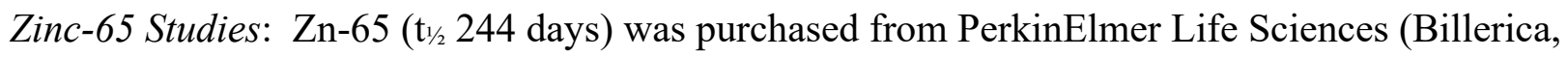
MA USA). One hour before administration of radiotracer, plants were removed from their hydroponics cells and suspended in $600-\mathrm{mL}$ beakers consisting of $100 \mathrm{~mL}$ of deionized water

(Figure 4.5.1.). Plants were maintained at the same daytime light and temperature conditions as that used to maintain their growth. An aqueous solution of $\mathrm{Zn}-65$ radiotracer at $0.74 \mathrm{MBq}$ was injected into the beaker of water in which the roots were immersed. A radiation detector (Eckler \& Ziegler, Inc., Berlin, Germany 1-inch NaI(Tl), photomultiplier tube gamma radiation detector) affixed to the plant $8 \mathrm{~cm}$ above the base of the stem provided dynamic feedback on $\mathrm{Zn}-65$ transport from roots to shoots. Data was acquired at $1 \mathrm{~Hz}$ sampling rate using 0-1 $\mathrm{V}$ analog input into an acquisition box (SRI, Inc, Torrance, CA, USA). After 3 hours of acquisition, roots were cut from the shoots, thoroughly washed in water, blotted dry and weighed. Shoots were also weighed. Both root and shoot tissues were then sequentially placed in a 3-inch $\mathrm{NaI}(\mathrm{Tl})$ gamma well-type detector for quantifying the amount of $\mathrm{Zn}-65$ radioactivity.

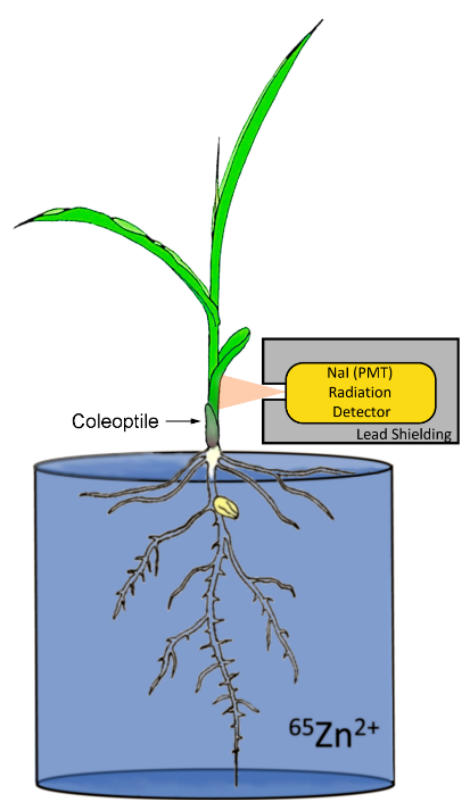

Figure 4.5.1. Experimental setup used for plant $\mathrm{Zn}-65$ uptake studies. During exposure to radiotracer, plants were maintained at a constant $500 \mu \mathrm{mol} \mathrm{m} \mathrm{m}^{-1}$ light intensity and $21^{\circ} \mathrm{C}$ temperature.

Plant Radiography: After Zn-65 administration plants were harvested, roots were blotted dry and laid out on an absorbent pad for imaging. Shoots were also laid out on a separate absorbent pad for imaging. Radiographic images of different tissue areas (roots and shoots) were acquired by exposing phosphor plate films. Phosphor plates of roots were exposed for 36 hours while plates 
of shoots were exposed for 120 hours to acquire a sufficient signal. After exposure, phosphor plates were then read using the Typhoon 9000 imager (Typhoon ${ }^{\mathrm{TM}}$ FLA 9000, GE Healthcare, Piscataway, NJ , USA). Images were only used qualitatively for determining spatial patterning of $\mathrm{Zn}-65$ tracer in roots and shoots, hence no attempt was made to normalize image data. Comparative whole-plant radiographic images of ${ }^{59} \mathrm{Fe}(\mathrm{II})$ and $\left.{ }^{59} \mathrm{FeIII}\right)$ were also acquired from our prior work ${ }^{15}$, but because of the faster decay rate of this radionuclide ( $t \frac{\mathrm{t} / 2}{44.5}$ days) we only exposed these tissues for 16 hours.

Ion Chromatography Analysis of Corn Kernel Zn Content: $\mathrm{Zn}$ content was quantified from corn kernels using ion chromatography coupled with UV absorption detection following the collection and drying of the kernels in an oven for 3 weeks at $65^{\circ} \mathrm{C}$. Seeds were pulverized between plastic sheets using a wooden mallet and dissolved in $1 \mathrm{~mL}$ of $1 \mathrm{M} \mathrm{HCl}$. Samples were subjected to ultrasonication for 5 minutes at 100\% amplitude (Branson Bransonic 32; Sigma-Aldrich Corp.

St. Louis, MO, USA) then centrifuged for 15 minutes at $3000 \mathrm{rpm}$. The supernatant was removed for sampling and stored in brown glass vials in a refrigerator $\left(2-8{ }^{\circ} \mathrm{C}\right)$. Zn standards were prepared in $0.1 \mathrm{M} \mathrm{HCl}$ using zinc chloride $\left(\mathrm{ZnCl}_{2}, 1 \mathrm{mg} \mathrm{mL}^{-1}\right)$.

The analytical system consisted of a Thermo Scientific Dionex AXP Metal-Free HPLC with a Rheodyne metal-free injector and PEEK tubing $1 / 20 \mathrm{~cm}$ inner diameter. The ion chromatography column was a Thermo Fisher Scientific ${ }^{\mathrm{TM}}$ Dionex $^{\mathrm{TM}}$ (Waltham MA, USA) IonPac CS5A $4 \times 250 \mathrm{~mm}$ analytical column outfitted with a CG5A $4 \times 40 \mathrm{~mm}$ guard column designed to separate a broad range of metal complexes by cation and anion chromatography. The isocratic mobile phase consisted of $7.0 \mathrm{mM}$ pyridine-2,6-dicarboxylic acid, $66 \mathrm{mM}$ potassium sulfate, $74 \mathrm{mM}$ formic acid $\mathrm{pH} 4.2$ run at a flow rate of $1.2 \mathrm{~mL} \mathrm{~min}^{-1}$. A post column reagent comprised of 0.5 mM 4-(2-pyridylazo) resorcinol (Dionex) in MetPac PAR post column diluent 
(1.0 M 2-dimethylaminoethanol/0.50 M ammonium hydroxide/0.30 M sodium bicarbonate $\mathrm{pH}$ 10.4) at a flow rate of approximately $0.6 \mathrm{~mL} \mathrm{~min}^{-1}$ was used for detection by a Knauer Smartline 2500 UV detector operated at $530 \mathrm{~nm}$. Sterile water (HyPure ${ }^{\mathrm{TM}}$ WFI Quality Water, HyClone Laboratories, Logan, UT, USA) was used in solvent preparation. All biological samples were analyzed in triplicate.

Statistical Analysis: Data was subjected to the Shapiro-Wilk Normality Test to identify outliers, so all data groups reflected normal distributions. Data was analyzed using the Student's t-test for pair-wise comparisons made between non-inoculated controls and bacteria treatment. Statistical significance was set at $P<0.05$.

Principal Component Analysis of Zn-65 and Fe-59 Data: The Zn-65 uptake and allocation data from the present study and Fe-59 data from our prior work ${ }^{16}$ were analyzed by Principal Component Analysis (PCA) using XLSTAT software version 2020.3 (Addinsoft Inc., New York, NY 10001, USA).

\subsection{Abbreviations}

\section{PGPB Plant growth promoting bacteria}

PCA Principal component analysis

PC Principal component 


\section{References}

(1) Marschner, H. Mineral Nutrition of Higher Plants, 2nd ed.; Academic Press: New York, NY, USA, 1995.

(2) Swietlik, D. Zinc Nutrition of Fruit Crops. HortTechnology 2002, 12 (1), 45-50.

(3) Fox, T. C.; Guerinot, M. L. MOLECULAR BIOLOGY OF CATION TRANSPORT IN PLANTS. Annu Rev Plant Physiol Plant Mol Biol 1998, 49, 669-696.

(4) Albion. Metalosate (R) Zinc in Plant Nutrition

http://www.albionplantnutrition.com/news/english-archives/doc_download/229-iron-nutritionand-metalosater-iron.

(5) Alloway, B. J. Soil Factors Associated with Zinc Deficiency in Crops and Humans. Environ Geochem Health 2009, 31 (5), 537-548.

(6) Cakmak, I. Enrichment of Cereal Grains with Zinc: Agronomic or Genetic Biofortification? Plant and Soil 2008, 302 (1/2), 1-17.

(7) Cavagnaro, T. R. The Role of Arbuscular Mycorrhizas in Improving Plant Zinc Nutrition under Low Soil Zinc Concentrations: A Review. Plant and Soil 2008, 304 (1/2), 315-325.

(8) Zhu, J.-K. Salt and Drought Stress Signal Transduction in Plants. Annu Rev Plant Biol 2002, 53, 247-273. https://doi.org/10.1146/annurev.arplant.53.091401.143329.

(9) Hess, S. Y. National Risk of Zinc Deficiency as Estimated by National Surveys. Food Nutr Bull 2017, 38 (1), 3-17.

(10) Goswami, D.; Thakker, J. N.; Dhandhukia, P. C. Portraying Mechanics of Plant Growth Promoting Rhizobacteria (PGPR): A Review. null 2016, 2 (1), 1127500.

(11) Yang, J.; Kloepper, J. W.; Ryu, C.-M. Rhizosphere Bacteria Help Plants Tolerate Abiotic Stress. Trends in Plant Science 2009, 14 (1), 1-4.

(12) Sarma, B. K.; Yadav, S. K.; Singh, D. P.; Singh, H. B. Rhizobacteria Mediated Induced Systemic Tolerance in Plants: Prospects for Abiotic Stress Management. In Bacteria in Agrobiology: stress management; Springer, 2012; pp 225-238.

(13) Pérez-Montaño, F.; Alías-Villegas, C.; Bellogín, R. A.; del Cerro, P.; Espuny, M. R.; Jiménez-Guerrero, I.; López-Baena, F. J.; Ollero, F. J.; Cubo, T. Plant Growth Promotion in Cereal and Leguminous Agricultural Important Plants: From Microorganism Capacities to Crop Production. Microbiological Research 2014, 169 (5-6), 325-336.

(14) Pankievicz, V. C. S.; do Amaral, F. P.; Santos, K. F. D. N.; Agtuca, B.; Xu, Y.; Schueller, M. J.; Arisi, A. C. M.; Steffens, M. B. R.; de Souza, E. M.; Pedrosa, F. O.; Stacey, G.; Ferrieri, R. A. Robust Biological Nitrogen Fixation in a Model Grass-Bacterial Association. Plant J 2015, 81 (6), 907-919.

(15) Backer, R.; Rokem, J. S.; Ilangumaran, G.; Lamont, J.; Praslickova, D.; Ricci, E.;

Subramanian, S.; Smith, D. L. Plant Growth-Promoting Rhizobacteria: Context, Mechanisms of 
Action, and Roadmap to Commercialization of Biostimulants for Sustainable Agriculture. Front. Plant Sci. 2018, 9.

(16) Housh, A. B.; Powell, G.; Scott, S.; Anstaett, A.; Gerheart, A.; Benoit, M.; Waller, S.; Powell, A.; Guthrie, J. M.; Higgins, B.; Wilder, S. L.; Schueller, M. J.; Ferrieri, R. A. Functional Mutants of Azospirillum Brasilense Elicit Beneficial Physiological and Metabolic Responses in Zea Mays Contributing to Increased Host Iron Assimilation. The ISME Journal 2021, 1-18.

(17) Reinhold, B.; Hurek, T.; Niemann, E.-G.; Fendrik, I. Close Association of Azospirillum and Diazotrophic Rods with Different Root Zones of Kallar Grass. Appl Environ Microbiol 1986, 52 (3), 520-526.

(18) Hurek, T.; Reinhold-Hurek, B.; Turner, G. L.; Bergersen, F. J. Augmented Rates of Respiration and Efficient Nitrogen Fixation at Nanomolar Concentrations of Dissolved O2 in Hyperinduced Azoarcus Sp. Strain BH72. J Bacteriol 1994, 176 (15), 4726-4733.

(19) James, E.; Olivares, F.; Baldani, J.; Dobereiner, J. Herbaspirillum, an Endophytic Diazotroph Colonizing Vascular Tissue in Leaves of Sorghum Bicolor L. Moench. Journal of Experimental Botany 1997, 48, 785-797.

(20) James, E. K.; Olivares, F. L. Infection and Colonization of Sugar Cane and Other Graminaceous Plants by Endophytic Diazotrophs. Critical Reviews in Plant Sciences 1998, 17 (1), 77-119.

(21) James, E.; Reis, V.; Olivares, F.; Baldani, J.; Dobereiner, J. Infection of Sugar Cane by the Nitrogen-Fixing Bacterium Acetobacter Diazotrophicus. Journal of Experimental Botany 1994, $45,757-766$.

(22) Okon, Y.; Labandera-Gonzalez, C. Agronomic Applications of Azospirillum - an Evaluation of 20 Years Worldwide Field Inoculation. Soil Biol Biochem 26:1591-1601 DOI 10.1016/0038-0717(94)90311-5. Soil Biology and Biochemistry 1994, 26, 1591-1601.

(23) Dobbelaere, S.; Croonenborghs, A.; Thys, A.; Ptacek, D.; Vanderleyden, J.; Dutto, P.; Labandera-Gonzalez, C.; Caballero-Mellado, J.; Aguirre, J. F.; Kapulnik, Y.; Brener, S.; Burdman, S.; Kadouri, D.; Sarig, S.; Okon, Y. Responses of Agronomically Important Crops to Inoculation with Azospirillum. Functional Plant Biol. 2001, 28 (9), 871-879.

(24) Hungria, M.; Campo, R. J.; Souza, E. M.; Pedrosa, F. O. Inoculation with Selected Strains of Azospirillum Brasilense and A. Lipoferum Improves Yields of Maize and Wheat in Brazil. Plant Soil 2010, 331 (1), 413-425.

(25) Zeffa, D. M.; Perini, L. J.; Silva, M. B.; Sousa, N. V. de; Scapim, C. A.; Oliveira, A. L. M. de; Júnior, A. T. do A.; Gonçalves, L. S. A. Azospirillum Brasilense Promotes Increases in Growth and Nitrogen Use Efficiency of Maize Genotypes. PLOS ONE 2019, 14 (4), e0215332.

(26) Reinhold-Hurek, B.; Hurek, T. Life in Grasses: Diazotrophic Endophytes. Trends in Microbiology 1998, 6 (4), 139-144. 
(27) Erenoglu, B.; Nikolic, M.; Römheld, V.; Cakmak, I. Uptake and Transport of Foliar Applied Zinc (65Zn) in Bread and Durum Wheat Cultivars Differing in Zinc Efficiency. Plant and Soil 2002, 241 (2), 251-257.

(28) Suzui, N.; Yin, Y.-G.; Ishii, S.; Sekimoto, H.; Kawachi, N. Visualization of Zinc Dynamics in Intact Plants Using Positron Imaging of Commercially Available 65Zn. Plant Methods 2017, $13(1), 40$.

(29) Youngdahl, L. J.; Svec, L. V.; Liebhardt, W. C.; Teel, M. R. Changes in the Zinc-65 Distribution in Corn Root Tissue with a Phosphorus Variable1. Crop Science 1977, 17 (1), cropsci1977.0011183X001700010019x.

(30) Ahmed, A. M. H.; Khalil, M. K.; Abd El-Rahman, A. M.; Nadia, A. M. H. Effect of Zinc, Tryptophan and Indole Acetic Acid on Growth, Yield and Chemical Composition of Valencia Orange Trees. Journal of Applied Sciences Research 2012, No. No.February, 901-914.

(31) Price, C. A. Molecular Approaches to Plant Physiology; McGraw-Hill: New York, 1970.

(32) Singh, M. Effect of Zinc, Phosphorus and Nitrogen on Tryptophan Concentration in Rice Grains Grown on Limed and Unlimed Soils. Plant and Soil 1981.

(33) Housh, A. B.; Powell, G.; Scott, S.; Anstaett, A.; Gerheart, A.; Benoit, M.; Waller, S.; Powell, A.; Guthrie, J. M.; Higgins, B.; Wilder, S. L.; Schueller, M. J.; Ferrieri, R. A. Functional Mutants of Azospirillum Brasilense Elicit Beneficial Physiological and Metabolic Responses in Zea Mays Contributing to Increased Host Iron Assimilation. The ISME Journal 2021, 1-18.

(34) Haga, K.; Iino, M. Auxin-Growth Relationships in Maize Coleoptiles and Pea Internodes and Control by Auxin of the Tissue Sensitivity to Auxin. Plant Physiology 1998, 117 (4), 14731486. 


\section{Chapter 5: Examining Effects of Rhizobacteria in Ameliorating Abiotic Crop Stresses using Carbon-11 Radiotracing}

\subsection{Preface}

This project was submitted on February $2^{\text {nd }}, 2021$ to the journal Frontiers in Plant Science. As third author on the manuscript, I was involved in the administration of the radiotracer to live plants and subsequent metabolite assays, measurement of potassium and calcium in the plant tissues via ICP-MS, starch assay method development, and edits on the final manuscript. All maize root measurements and completion of the starch assay experiments were done and contributed by Avery Powell and Stacy Wilder of the Plant Radiotracer research group within the University of Missouri Research Reactor Center.

\subsection{Introduction}

Plants are sessile organisms which encounter a variety of stresses at every developmental stage of their life including temperature extremes, drought, flooding, salinity and soil nutrient limitation due to $\mathrm{pH}$ shifts ${ }^{1}$. Today, approximately $30 \%$ of global crop production is lost as a result of abiotic stresses ${ }^{2}$. Salinity is considered one of the most critical abiotic stresses, impacting agricultural productivity and sustainability due to reductions in photosynthesis, respiration, and protein synthesis ${ }^{3-5}$. This is a growing problem, as the proportion of salinized land is expected to increase as climate change conditions encourage salt accumulation ${ }^{6}$. Soil salinity can result in widespread nutritional disturbances impacting plant growth due to effects on plant-water relations ${ }^{7,8}$ from the high concentrations of $\mathrm{Na}^{+}$ions in the soil and within the plant ${ }^{9}$. Plants have evolved adaptive mechanisms to deal with adverse effects of salinity, including the accumulation of solutes like amino acids, sugars, or counterions such as $\mathrm{K}^{+}$or quaternary ammonium $\left(\mathrm{R}-\mathrm{NH}_{4}{ }^{+}\right)$, that behave as potent osmolytes or osmoprotectants aiding in 
cellular maintenance of water status ${ }^{10}$. Often, though, these natural adaptions are not sufficient to address acute stress.

Nitrogen is also recognized as a major limiting input constraint for maize production ${ }^{11-}$ 14. Plants in natural fields face changing environmental conditions where $\mathrm{N}$ concentrations vary and are frequently limiting for growth due to surface run-off/soil erosion, rainwater leaching, gaseous losses by volatilization, and microbial consumption. Plant adaptation to limited $\mathrm{N}$ conditions is an important survival strategy enabling them to successfully complete their life cycle. Typically, a low $\mathrm{N}$ supply leads to decreased root growth with suppression of lateral root emergence, an increased $\mathrm{C} / \mathrm{N}$ ratio within tissues, which causes reduced photosynthesis, and early leaf senescence ${ }^{15-21}$.

Plant growth promoting bacteria (PGPB), which can help their host weather difficult conditions, are finding increased use in agriculture. These organisms can activate physiological and biochemical responses within their host for mutual benefit to build natural tolerances to environmental stresses and thereby reduce losses in the field ${ }^{22-27}$. A number of different PGPB commonly found in the plant rhizospheres can establish close associations with roots, colonizing either epiphytically or endophytically. Indeed, PGPB reach quite high numbers (e.g., 10 $/ \mathrm{gfw}$ ) in roots without inducing noticeable plant defense responses ${ }^{28-30}$. PGPB alter the host plant's metabolic and physiological state through direct and indirect actions $\mathrm{s}^{31-34}$. Direct actions of growth promotion include their ability to produce phytohormones, reduce plant ethylene levels, emit volatile organic compounds that can protect plants ${ }^{35-38}$, and enhance plant nutrient availability by releasing phosphates and micronutrients from insoluble soil sources and fixing non-symbiotic nitrogen $26,32,39$. Yet, while all these actions have been identified as contributing to 
the overall growth benefits seen in plants, we still know little of the mechanisms underpinning these actions.

Since PGPB are seen to be useful in modern agriculture as a mitigation strategy for reducing abiotic stress-related crop losses, we applied ${ }^{11} \mathrm{C}$ radiotracing to a relevant crop model, Zea mays, inoculated with a microbial agent to map changes in the physiological and biochemical responses. Among PGPB, the genus Azospirillum-with an emphasis on $A$. brasilense - is probably the most studied microorganism for the mitigation of plant stress, especially salinity stress ${ }^{40-44}$. Our recent success in demonstrating plant growth promotion using HM053, a functional mutant strain of $A$. brasilense in grasses ${ }^{45-47}$ led us to test its performance in mitigating two different abiotic stresses including salinity and nitrogen limitation. This work reports results on the amelioration of plant stresses imposed by salinity and nitrogen limitation through the actions of PGPB where the physiological and metabolic responses of the host were examined in detail using carbon-11 radiotracing and correlated with root growth traits.

\subsection{Results}

Root Phenotype: Because certain abiotic stresses are known to affect root growth and root architecture, we wanted to examine root growth traits as a function of salt exposure and nitrogen limitation as well as the combination of these abiotic stresses with administration of the microbial inoculant $A$. brasilense (HM053). Results in Figure 5.3.1. show a representative sampling of maize root images from plants grown under the different experimental conditions

outlined above. Chronic exposure to salt during growth (Figure 5.3.1. B) resulted in shorter roots compared to those under normal growth conditions (Figure 5.3.1. A), which were quantified (Figure 5.3.1. F). Though not measured, higher density fine root growth was observed under salinity stress than under normal growth conditions. This behavior resulted in a significantly 
higher root mass (Figure 5.3.1. G)

relative to normal growth conditions.

The introduction of HM053 $A$.

brasilense inoculant onto this stress

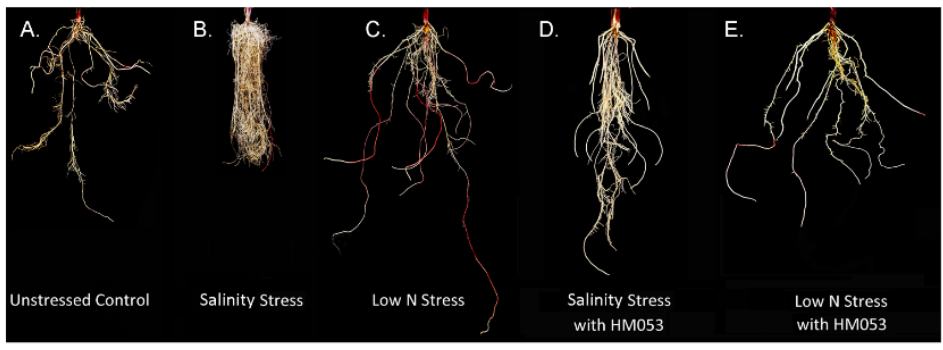

resulted in a reduction in the fine root

phenotype with increased root length

(Figure 5.3.1. F) relative to non-

inoculated stressed plants which

correlated with normal unstressed root
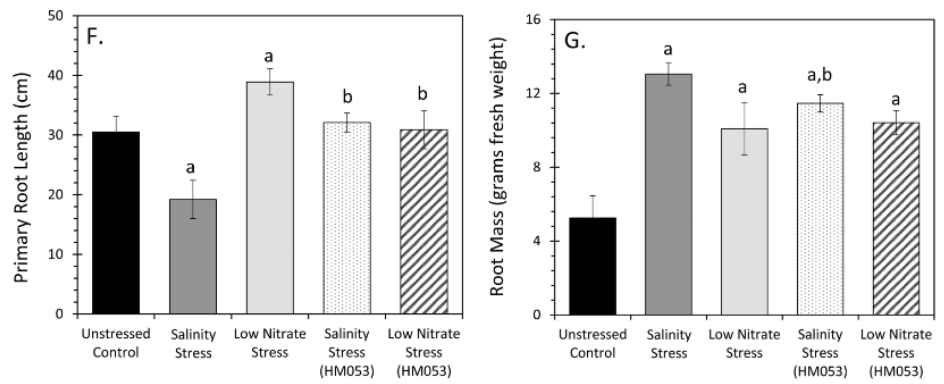

Figure 5.3.1. Root Characteristics of Maize under Abiotic Stress. Panels A-E: Photographs reflect the growth. Taken together, root mass

remained significantly elevated relative

to normal growth conditions.

Contrary to the above observations, nitrogen limitation caused longer roots to grow (Figure 5.3.1. C\&F) relative different growth traits of maize roots when plants were grown under the different study conditions including salinity stress and low nitrogen stress with and without inoculation using HM053 A. brasilense. Panel F: Root length was measured using ImageJ software. Panel G: Root fresh mass was weighed using an analytical balance. Data $( \pm \mathrm{SE})$ reflects $\mathrm{N}=6-9$ biological replicates. Bars in Panel F-G denoted with an "a" reflect statistical significance $(\mathrm{P}<0.05)$ in a comparison between the stressed stated and unstressed control. Bars denoted with an " $\mathrm{b}$ " reflects statistical significance $(\mathrm{P}<0.05)$ in a comparison between inoculated and non-inoculated stressed plants.

to normal growth conditions, which manifested in a significantly higher root mass (Figure 5.3.1. G). It was noted that roots also had a strong red pigmentation when grown under nitrogen limiting conditions. The introduction of HM053 A. brasilense inoculant onto this stress reduced root length (Figure 5.3.1. F) returning the phenotype back to normal unstressed root growth. Also, the red root pigmentation was less pronounced in the presence of the microbial inoculant (Figure 5.3.1. E) and root mass remained significantly elevated (Figure 5.3.1. G).

Plant Physiological Responses: Using carbon-11 administered to plants as $\left[{ }^{11} \mathrm{C}\right] \mathrm{CO}_{2}$, the following physiological traits were measured for the above growth conditions: (i) leaf fixation 
(Figure 5.3.2. A); (ii) leaf export of ${ }^{11} \mathrm{C}$-photoassimilates (Figure 5.3.2. B); (iii) allocation of ${ }^{11} \mathrm{C}$-photoassimilates to root tissues (Figure 5.3.2. C); and (iv) exudation of ${ }^{11} \mathrm{C}$-substrates (Figure 5.3.2. D) as acidic (A) and non-acidic (N) compounds. For leaf fixation, data was presented as percent of ${ }^{11} \mathrm{C}$-activity in the pulse. In all other cases, data was presented as a percent of the ${ }^{11} \mathrm{C}$-activity fixed by the plant. Results indicated that salinity stress had no impact on leaf $\left[{ }^{11} \mathrm{C}\right] \mathrm{CO}_{2}$ fixation $(67 \pm 6 \%)$ relative to non-inoculated unstressed plants $(62 \pm 6 \%)$. The administration of the microbial inoculant onto this stress did not change this behavior $(61 \pm 6 \%)$. Contrary to this, nitrogen limitation significantly decreased $\left[{ }^{11} \mathrm{C}\right] \mathrm{CO}_{2}$ fixation $(41 \pm 4 \%)$ relative to non-inoculated unstressed plants, while introduction of the microbial inoculant under nitrogen limitation significantly increased $\left[{ }^{11} \mathrm{C}\right] \mathrm{CO}_{2}$ fixation $(60 \pm 5 \%)$ relative to non-inoculated plants grown under the same stress condition reinstating normal physiological behavior.

Similarly, salinity stress had no effect on leaf export of ${ }^{11} \mathrm{C}$-photoassimilates $(60 \pm 5 \%)$ relative to non-inoculated unstressed plants $(55 \pm 3 \%)$, nor did the introduction of the microbial inoculant alter this behavior (61 $\pm 7 \%)$. Nitrogen limitation, however, significantly decreased leaf export $(40 \pm 6 \%)$ relative to non-inoculated unstressed plants while introduction of the microbial inoculant onto this stress significantly increased leaf export $(59 \pm 3 \%)$ relative to stressed noninoculated plants, reinstating normal unstressed physiological behavior.

Further, salinity stress significantly increased allocation of ${ }^{11} \mathrm{C}$-photoassimilates to root tissues $(43 \pm 3 \%)$ relative to non-inoculated unstressed plants $(24 \pm 3 \%)$. Introduction of the microbial inoculant here showed similar elevated responses $(50 \pm 5 \%)$ as non-inoculated stressed plants. Contrary to this, nitrogen limitation significantly decreased root allocation $(10 \pm 2 \%)$ relative to non-inoculated unstressed plants while the introduction of microbial inoculant onto 
this stress significantly increased allocation $(38 \pm 3 \%)$ relative to non-inoculated stressed plants reinstating and even surpassing normal unstressed physiological behavior.
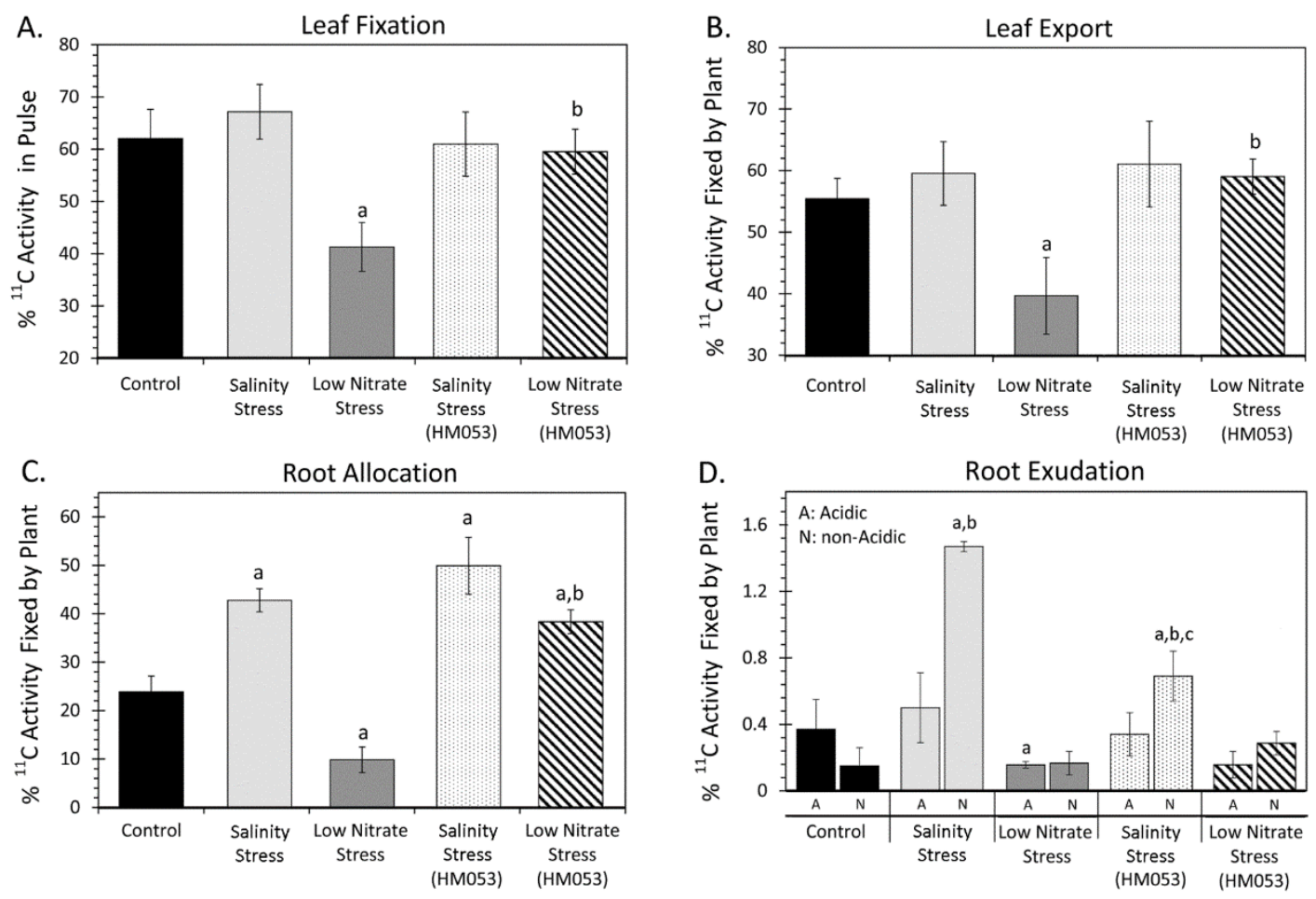

Figure 5.3.2. Carbon-11 aids in mapping maize physiological responses to applied abiotic stresses of salinity and nitrogen limitation and to the introduction of microbial inoculant onto these stresses. Panel A: Leaf tissue normalized ${ }^{11} \mathrm{CO}_{2}$ fixation presented as $\%{ }^{11} \mathrm{C}$-activity in the pulse applied to the leaf cuvette. Panel B: Leaf export of ${ }^{11} \mathrm{C}$-photosynthates (measured at $1.5 \mathrm{hr}$. post ${ }^{11} \mathrm{CO}_{2}$ pulse) presented as \% fixed ${ }^{11} \mathrm{C}$-activity by the plant. Panel C: Root allocation of ${ }^{11} \mathrm{C}$ photosynthates presented as \% fixed ${ }^{11} \mathrm{C}$-activity by the plant. For Panels A-C, data $( \pm \mathrm{SE})$ reflects $\mathrm{N}=6-9$ biological replicates. Bars in these panels denoted with an "a" reflect a statistical significance $(\mathrm{P}<0.05)$ in a comparison between stressed and unstressed plants. Bars denoted with an "b" reflect a statistical significance $(\mathrm{P}<0.05)$ in a comparison between non-inoculated and inoculated stressed plants. Panel D: Root exudation of ${ }^{11} \mathrm{C}$-substrates $(\mathrm{A}=$ acidic substrates; $\mathrm{N}=$ non-acidic substrates measured at $1.5 \mathrm{hr}$. post ${ }^{11} \mathrm{CO}_{2}$ pulse) presented as \% fixed ${ }^{11} \mathrm{C}$-activity by the plant. For Panel D, data $( \pm \mathrm{SE})$ reflects $\mathrm{N}=6-9$ biological replicates. Bars in this panel denoted with an "a" reflect a statistical significance $(\mathrm{P}<0.05)$ in a comparison between the stressed state and unstressed control state for same category of exudate. Bars denoted with an " $b$ " reflect a statistical significance $(\mathrm{P}<0.05)$ in a comparison between different category of exudate for same plant treatment. Bars denoted with an "c" reflect a statistical significance $(\mathrm{P}<0.05)$ in a comparison between same category of exudate in a comparison between inoculated and non-inoculated plants.

Non-inoculated unstressed plants exhibited approximately equal portions of ${ }^{11} \mathrm{C}$-labeled acidic and non-acidic exudates $(0.37 \pm 0.21 \%$ and $0.15 \pm 0.11 \%$, respectively). Salinity stress, however, caused a significant shift in this behavior favoring significantly higher non-acidic 
exudates $(1.47 \pm 0.09 \%)$, with no significant change in the acidic fraction $(0.50 \pm 0.22 \%)$. Introduction of the microbial inoculant onto this stress reduced the non-acidic fraction $(0.69 \pm 0.19 \%)$ somewhat though this fraction was still significantly higher than unstressed plants. No change was observed in the acidic fraction under these conditions $(0.34 \pm 0.15 \%)$. Low nitrogen stress significantly reduced exudation of acidic ${ }^{11} \mathrm{C}$-substrates $(0.16 \pm 0.05 \%)$ relative to non-inoculated unstressed plants. Introduction of the microbial inoculant onto this stress did not affect the acidic fraction $(0.16 \pm 0.10 \%)$, but increased the non-acidic fraction $(0.29 \pm 0.10 \%)$ surpassing levels seen in non-inoculated unstressed plants.

${ }^{11}$ C-Metabolite Profiling: Often, metabolic profiling of how plants use their newly acquired carbon resources can provide insight into the state of the plant, as well as insight into mechanisms of action when PGPB inoculants are introduced during growth to alter their host's metabolic state ${ }^{47}$. Here we profiled the metabolic landscape of newly fixed $\left[{ }^{11} \mathrm{C}\right] \mathrm{CO}_{2}$ as that carbon source was quickly partitioned between the different metabolite pools of the leaf tissue. In unstressed non-inoculated plants (Figure 5.3.3. A) partitioning of new carbon generated the following baseline profile: structural and hydrophobic metabolites (16.7 $\pm 1.1 \%)$; soluble sugars (32.6 $\pm 3.1 \%)$; amino acids (4.0 $\pm 0.6 \%)$; basic metabolites $(32.1 \pm 2.1 \%)$; acidic metabolites $(14.4 \pm 1.8 \%)$ excluding amino acids. Salinity stress imposed no significant change in this partitioning distribution with the exception that the acidic metabolite pool was significantly decreased $(9.4 \pm 1.1 \%)$ relative to non-inoculated unstressed plants. Introduction of the microbial inoculant onto this stress resulted in a significant increase in the soluble sugar pool $(44.5 \pm 1.6 \%)$, and significant decreases in the amino acid pool $(1.4 \pm 0.4 \%)$ and basic metabolite pool $(18.4 \pm 3.8 \%)$ relative to non-inoculated unstressed plants. The acidic metabolite pool was increased $(13.0 \pm 0.1 \%)$ to a level commensurate with that of non-inoculated unstressed plants. 
A.
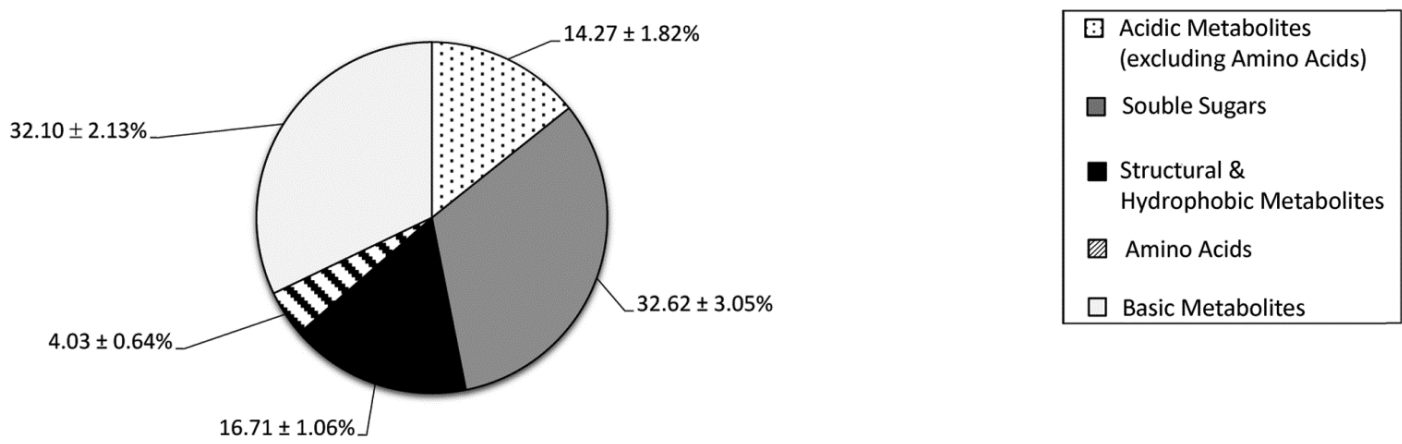

B.

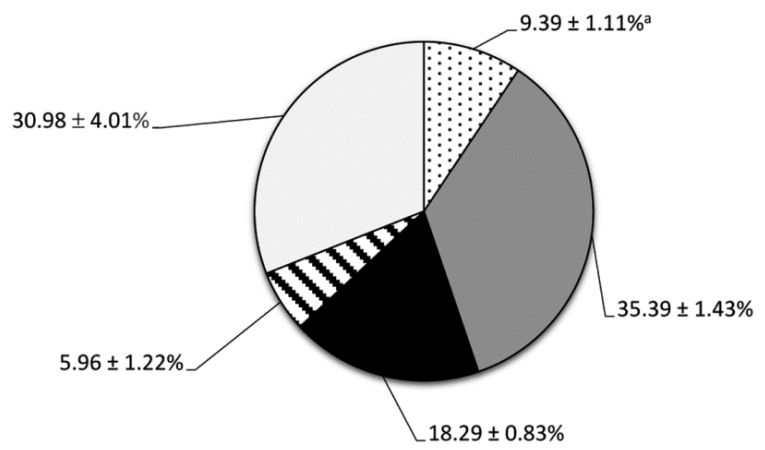

D.

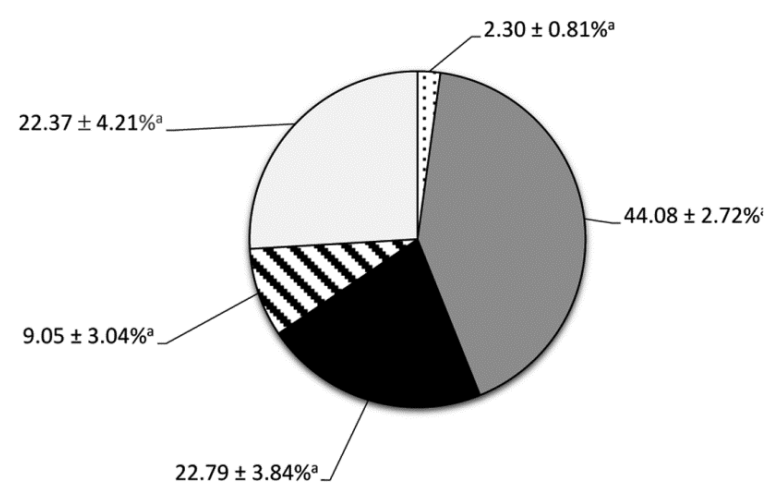

C.

Salinity Stress - HM053

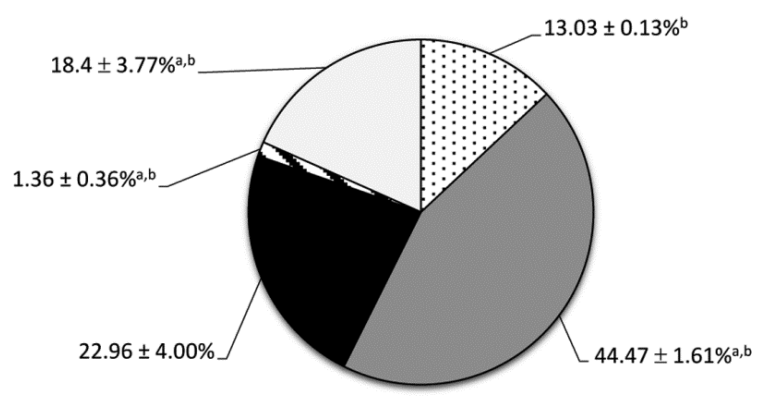

E. Low Nitrate Stress - HM053

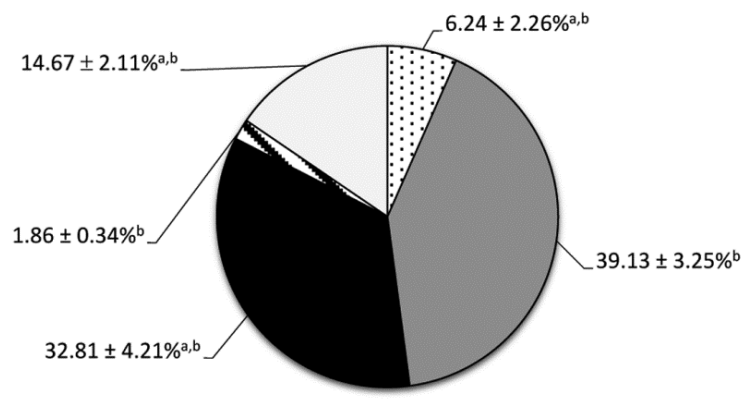

Figure 5.3.3. Carbon-11 aids in mapping changes in maize carbon metabolism as a function of applied abiotic stresses of salinity and nitrogen limitation and to the introduction of microbial inoculant onto these stresses. The metabolic landscape reflects the partitioning of 'new' carbon (as ${ }^{11} \mathrm{C}$ ) into different metabolic pools of the load leaf tissue when harvested 20 minutes after the initial ${ }^{11} \mathrm{CO}_{2}$ pulse. Data $( \pm \mathrm{SE})$ reflects $\mathrm{N}=6-9$ biological replicates. Pie sections notated with an "a" reflect statistical significance $(\mathrm{P}<0.05)$ in a comparison between the stressed stated and unstressed control. Pie sections denoted with an " $\mathrm{b}$ " reflect statistical significance $(\mathrm{P}<0.05)$ in a comparison between inoculated and non-inoculated stressed plants. 
Stress imposed by nitrogen limitation presented a very different profile in new carbon partitioning than salinity stress. Results here showed a significant increase in the

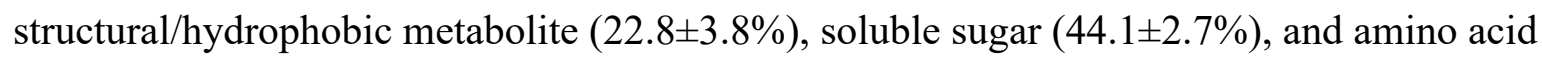
$(9.1 \pm 3.0 \%)$ pools, and significant decreases in the basic metabolite $(22.4 \pm 4.2 \%)$ and acidic metabolite $(2.3 \pm 0.8 \%)$ pools relative to non-inoculated unstressed plants. Introduction of the microbial inoculant onto nitrogen stressed plants resulted in a further significant increase in the structural/hydrophobic pool (32.8 $\pm 4.2 \%)$. The soluble sugar pool was significantly reduced $(39.1 \pm 3.3 \%)$ from that of non-inoculated stressed plants bringing it in line with the behavior of non-inoculated unstressed plants. Further, the amino acid pool was significantly reduced in the presence of inoculant $(1.9 \pm 0.3 \%)$ below that of non-inoculated unstressed plants. Finally, basic

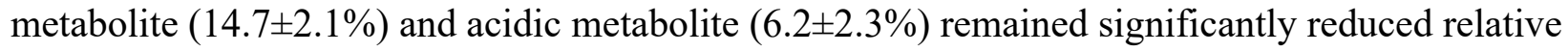
to unstressed plants.

Starch Analysis: Several of the physiological responses highlighted above prompted the question about what carbon resources are being utilized in support of root growth when plants are subjected to abiotic stresses imposed by salinity or nitrogen limitation. For this reason, shoots and roots of plants were subjected to starch analysis as a function of the imposed growth conditions (Figure 5.3.4.). Non-inoculated unstressed plants exhibited levels of starch in leaves and root of $477.0 \pm 104.9$ and $20.7 \pm 5.0 \mathrm{mg} \mathrm{g}^{-1} \mathrm{DW}$ (DW: dry weight), respectively. Consistent with the literature, root starch levels were always significantly less than leaf starch levels, regardless of the growth conditions (Ning et al., 2018; Wu et al., 2019). Stress from salinity caused significant reduction in leaf starch levels $\left(54.2 \pm 32.5 \mathrm{mg} \mathrm{g}^{-1} \mathrm{DW}\right)$ and elevation in root starch levels (34.0 $\left.\pm 4.2 \mathrm{mg} \mathrm{g}^{-1} \mathrm{DW}\right)$ relative to non-inoculated unstressed plants. Introduction of microbial inoculant onto this stress continued to cause suppressed leaf starch levels $(73.7 \pm 7.5 \mathrm{mg}$ 
$\mathrm{g}^{-1} \mathrm{DW}$ ) relative to non-inoculated unstressed plants, while root starch continued to increase significantly (45.2 $\left.\pm 3.1 \mathrm{mg} \mathrm{g}^{-1} \mathrm{DW}\right)$ relative to non-inoculated stressed plants and unstressed

plants. Stress from nitrogen limitation had no significant impact on leaf starch levels (457.3 $\left.\pm 108.5 \mathrm{mg} \mathrm{g}^{-1} \mathrm{DW}\right)$, but significantly reduced root starch levels $\left(11.9 \pm 2.8 \mathrm{mg} \mathrm{g}^{-1} \mathrm{DW}\right)$ relative to non-inoculated unstressed plants. Interestingly, introduction of the microbial inoculant onto this stress caused significant increases in both leaf starch $\left(617.8 \pm 146.8 \mathrm{mg} \mathrm{g}^{-1}\right.$ DW) and root starch $\left(57.3 \pm 13.1 \mathrm{mg} \mathrm{g}^{-1} \mathrm{DW}\right)$ levels.

\section{ICP-MS Analysis of Plant Potassium and}

Calcium: We examined whether HM053 A.

brasilense could influence levels of certain ions within the host that are important in regulating salinity stress. Potassium, a potent osmolyte ${ }^{57}$, was examined along with calcium - the latter being implicated in signaling processes that trigger plant adaptive responses to salinity ${ }^{58}$. Results in Figure

5.3.5. A indicate that roots had consistently a significantly higher level of potassium $(45,025 \pm 1,049 \mathrm{ppm})$ than shoots $(33,733 \pm 1,004$
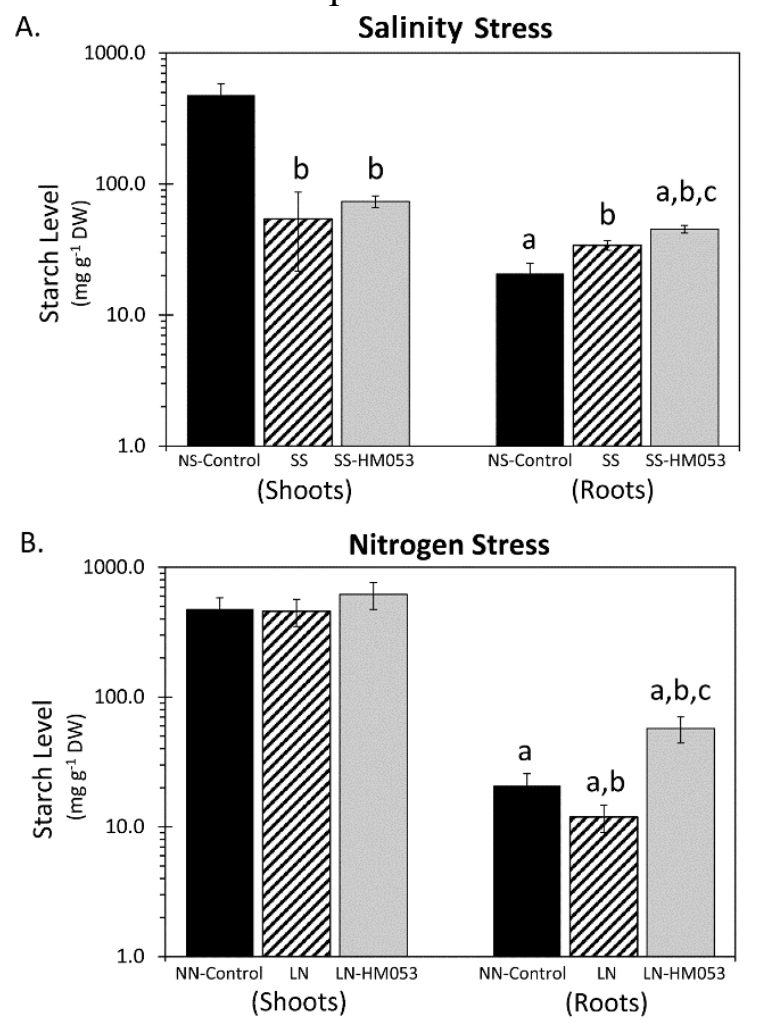

Figure 5.3.4. Tissue starch levels are presented for shoots and root tissues as a function of salinity stress. (Panel A) and low nitrogen stress (Panel B) and as a function of inoculation with HM053 $A$. brasilense. (NS, no salt; SS, salt stress; NN, normal nitrogen; LN, low nitrogen). Data $( \pm \mathrm{SE})$ reflects $\mathrm{N}=9-12$ biological replicates. Bars in these panels denoted with an "a" reflect a statistical significance $(\mathrm{P}<0.05)$ in a comparison between tissue types for the different growth conditions. Bars denoted with an "b" reflect a statistical significance $(\mathrm{P}<0.05)$ in a comparison between same tissue types of stressed and unstressed plants. Bars denoted with an "c" reflect a statistical significance $(\mathrm{P}<0.05)$ in a comparison between same tissue types of stressed plants that were either noninoculated or inoculated with bacteria. 
ppm) within non-inoculated unstressed plants. Our data was somewhat different from prior published work ${ }^{59}$ indicating between 1.6-to-2.3 times lower potassium levels overall, with roots containing lower levels than shoots (roots, $\sim 19,000$ ppm; shoots, $\sim 27,000$ ppm). The differences
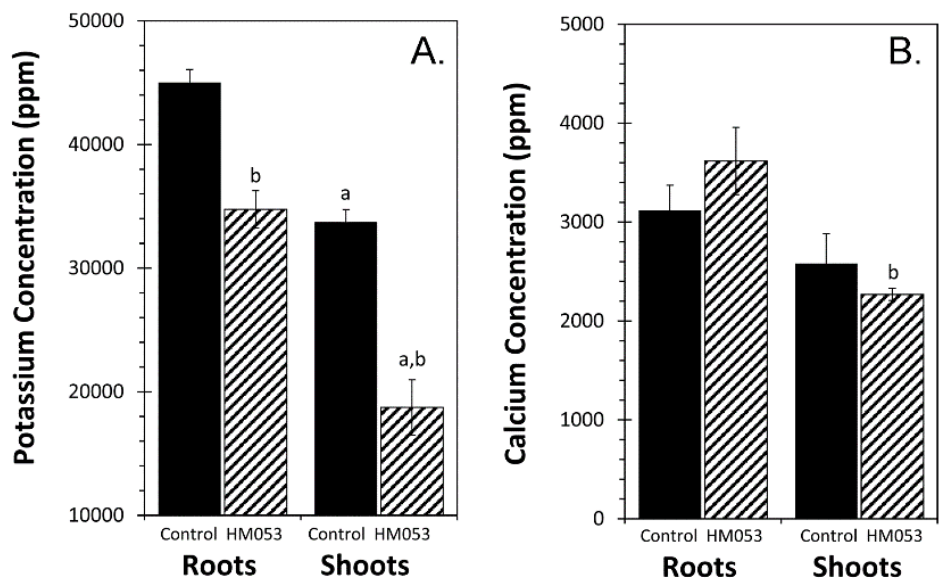

here may be due to the way plants

were grown. Introduction of

microbial inoculant during plant

growth resulted in significant

reductions in root and shoot

potassium to levels of $34,750 \pm 1,520$

Figure 5.3.5. ICP-MS data shows levels of $\mathbf{K}^{+}$(Panel A.) $\quad$ ppm and 18,733 $\pm 2,236$ ppm, and $\mathrm{Ca}^{2+}$. (Panel B.) in shoot and root tissues of noninoculated and HM053 A. brasilense inoculated plants. Data $( \pm \mathrm{SE})$ reflects $\mathrm{N}=6-9$ biological replicates. Bars in these panels denoted with an "a" reflect a statistical significance $(\mathrm{P}<0.05)$ in a comparison between tissue types for the different growth conditions. Bars denoted with an "b" reflect a statistical significance $(\mathrm{P}<0.05)$ in a comparison between same tissue types of non-inoculated and inoculated plants.

respectively.

Additionally, results in Figure 5.3.5.

$\mathbf{B}$ indicate that roots had a

consistently higher level of calcium $(3,120 \pm 250 \mathrm{ppm})$ than shoots $(2,270 \pm 300 \mathrm{ppm})$ in non-inoculated unstressed plants though not

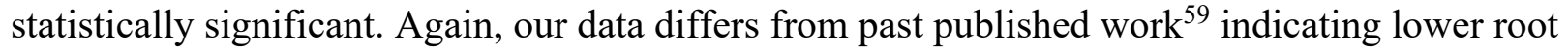
levels $(\sim 3,000 \mathrm{ppm})$ in comparison to shoots $(\sim 12,000 \mathrm{ppm})$. Introduction of microbial inoculant during plant growth did not alter host calcium levels (roots, 3,617 \pm 340 ppm; shoots, 2,270 \pm 60 ppm).

\subsection{Discussion}

Amelioration of salinity stress symptoms using HM053 A. brasilense: Excessive salt stress can restrict plant growth due to induced ionic, osmotic, or even oxidative stresses tied to the high levels of sodium ions taken up by the plant ${ }^{60,61}$. Since a plant's root system is the major organ 
responsible for uptake of water and nutrients from the soil, understanding their function becomes crucial for enhancing plant resistance to salt stress as they are the gateway to regulating $\mathrm{Na}^{+}$ acquisition and translocation ${ }^{62}$. Typically, in the presence of high concentrations of $\mathrm{Na}^{+}$ions, plants will experience a decline in primary root growth due to inhibition of cell division and elongation of root epidermal cells $\mathrm{s}^{62,63}$ with compensatory growth stimulation of lateral roots. Here fine root systems are better equipped to allow plants to track non-saline areas of the soil for water, minerals or other needed resources until exploitation of heavy salt concentrated areas become unavoidable ${ }^{64-66}$.

In the present work, a similar root phenotype was exhibited where saline stressed plants exhibited shorter primary roots and stimulated growth of fine roots resulting in an increase in total root biomass relative to unstressed plants. Although no change in plant carbon input via $\left[{ }^{11} \mathrm{C}\right] \mathrm{CO}_{2}$ fixation was observed with this stress, nor a change in export of leaf ${ }^{11} \mathrm{C}$ photosynthates, a higher portion of this mobile new carbon source was allocated to roots presumably in support of growth in that tissue. This would suggest less carbon was available to support stem growth. Although this feature was not examined in the present work, it was noted that starch levels in saline stressed roots were significantly higher than in unstressed roots, while starch levels in saline stressed shoots were significantly lower than in non-stressed shoots that could be attributable to the observed changes in carbon allocation.

Several past works have shown that the presence of certain microorganisms ${ }^{67}$ and arbuscular mycorrhizal fungi ${ }^{68,69}$ can mitigate the effects of salinity stress. In the present work, introduction of HM053 A. brasilense during maize growth under saline stress appeared to reinstate a root architecture like unstressed plants - i.e., longer primary roots were apparent with less fine root structure - suggesting some degree of amelioration of the host's stressed state. ICP- 
MS data suggests that a mechanism for microorganism induced tolerance to salt was not due to increased host uptake of osmolytes such as $\mathrm{K}^{+}$. In fact, HM053 reduced host $\mathrm{K}^{+}$uptake.

Furthermore, plant response to hyperosmotic tension caused by high levels of $\mathrm{Na}^{+}$can be closely linked to $\mathrm{Ca}^{+2}$ channels and $\mathrm{Ca}^{+2}$ sensing ${ }^{58}$. However, HM053 introduction in the absence of salt stress did not alter host $\mathrm{Ca}^{+2}$ levels. It is acknowledged that such measurements say little about whether cellular $\mathrm{Ca}^{+2}$-signaling was altered, but such measurements were beyond the scope of this project.

It was noted that introduction of this microbial inoculant significantly increased the partitioning of 'new' carbon into the soluble sugar pools at the expense of amino acid and other basic metabolite biosynthesis. Such alteration in metabolic sugar regulation corresponded well with the observed increased transport of ${ }^{11} \mathrm{C}$-photosynthates at the whole-plant level, and with the increased exudation of non-acidic substrates from the roots. Many sugars will behave as potent osmolytes aiding in building cellular tolerance to hyperosmotic tension caused by high levels of $\mathrm{Na}^{+57}$. Hence, it is suspected this is the primary mechanism of action for microbial amelioration of salinity stress.

Amelioration of nitrogen deficiency symptoms using HM053 A. brasilense: The heterogeneous distribution of nitrogen in the soil has forced plants to adapt by altering their root architecture to enhance nutrient acquisition ${ }^{70}$. This plasticity of roots supports plant survival under non-ideal conditions and enables them to compete more effectively for resources when experiencing deficiencies of nitrogen or other essential nutrients ${ }^{71}$. Past studies have shown that the influences of nitrogen limitation on maize root growth traits can be very dependent on variety, but for the most part, roots were longer when subjected to this stress ${ }^{72}$. Consistent with this trend, the present study showed evidence of increased root length when plants were subjected to nitrogen 
limitation. The carbon source supporting this root growth was of particular interest. Results from the carbon- 11 studies revealed that $\left[{ }^{11} \mathrm{C}_{\mathrm{CO}_{2}}\right.$ fixation was reduced by $40 \%$ under nitrogen limiting conditions as were leaf export and root allocation of ${ }^{11} \mathrm{C}$-photosynthates. Hence, less 'new' carbon is available in support of root growth. It was noted, however, that root starch levels were significantly reduced in roots of nitrogen stressed plants relative to non-inoculated unstressed plants. This observation suggests that less carbon is placed into storage pools but rather partitioned into structural components in support of cell wall development. Interestingly, introduction of microbial inoculant onto the nitrogen limiting stress bolstered root starch levels surpassing that in unstressed plants. This seems reasonable given that root growth here was like that of unstressed plants.

The other important observation to note here is the loss of red pigmentation in roots of HM053 exposed plants grown under nitrogen limitation. The presence of anthocyanins is usually associated with red pigmentation of plant tissues. In past work involving Arabidopsis, nitrogen deficiency consistently induced transcript levels of key genes involved in anthocyanin biosynthesis, including $F 3^{\prime} H, D F R$, $L D O X$, and $U F 3 G T$ causing systemic accumulation of the metabolite $^{73}$. Likewise, the present study showed extensive red pigmentation of roots of plants grown under nitrogen limitation. Part of this action can be attributed to increased partitioning of new carbon $\left(\right.$ as $\left.{ }^{11} \mathrm{C}\right)$ into amino acids noting that anthocyanins are products of phenylalanine and tyrosine, which metabolize to cinnamic acid and coumaric acid, respectively, in the phenyl propanoid pathway. Anthocyanin biosynthesis is also induced by sucrose $\mathrm{e}^{74,75}$. In this work, nitrogen limited growth caused a significant increase in 'new' carbon $\left(\right.$ as $\left.{ }^{11} \mathrm{C}\right)$ partitioning into soluble sugars with sucrose dominating that pool. This change in carbon metabolism could be the reason for the red pigmented roots observed under nitrogen limiting growth. Furthermore, the 
introduction of the microbial inoculant onto this stress with the resultant reversal in the sugar pool back to a level commensurate with non-inoculated unstressed plants could account for the loss of root pigmentation.

As a diazotroph HM053 A. brasilense has been implicated with relieving nitrogen stress in setaria viridis grass by supplying extraneous nitrogen to its host via biological nitrogen fixation ${ }^{76}$. Whether or not a similar mechanism of action is in play for the maize system examined in the present work remains to be seen. Future studies using radioactive ${ }^{13} \mathrm{NN}$ to directly quantify host nitrogen uptake via biological nitrogen fixation will be needed ${ }^{46}$. Alternatively, this microbial inoculant could upregulate host nitrate uptake under nitrogen limiting growth conditions. Although less nitrogen is available in the growth medium, the increase in nitrate uptake kinetics might compensate for the deficiency. This too can be examined in future studies using the radioactive ${ }^{13} \mathrm{NO}_{3}{ }^{-}$tracer. Indeed, past studies in birch saplings using ${ }^{13} \mathrm{NO}_{3}{ }^{-}$showed an alteration in the tracer uptake kinetics with nitrate availability ${ }^{77}$.

\subsection{Conclusions}

The present work shows evidence that HM053 A. brasilense can act through many diverse mechanisms of action in the amelioration of plant abiotic stress symptoms caused by salinity and nitrogen limitation. Furthermore, this work showcases the power and utility of using carbon-11 radiotracing to map changes in whole-plant physiology and 'new' carbon metabolism giving insight into how these PGPB interact and influence the state of their host plant.

\subsection{Materials and Methods}

Plant Growth: Maize kernels from Elk Mound Seed Co. (Hybrid 8100) were dark germinated at room temperature for two days on sterilized paper towels wetted with sterile water in a petridish. Seeds were inoculated with bacteria culture as appropriate and transplanted to a growth 
pouch wetted with sterile Hoagland's basal salt solution for approximately one week. They were then transferred to eight-inch plastic cones filled with Turface ${ }^{\mathrm{TM}}$ (expanded clay matrix) where the bottom portion of the cone was immersed in de-ionized water. Nutrient was introduced as Hoagland's solution every three days. Growth conditions for both set-ups consisted of 12-hour photoperiods, $500 \mu \mathrm{mol} \mathrm{m} \mathrm{s}^{-2}$ light intensity, and temperatures of $25^{\circ} \mathrm{C} / 20^{\circ} \mathrm{C}$ (light/dark) with humidity at $70-80 \%$ for two weeks.

Plants subjected to salinity stress were grown as described above; however, cones were immersed in $100 \mathrm{mM} \mathrm{NaCl}$. Every $3^{\text {rd }}$ day $50 \mathrm{~mL}$ of Hoagland's nutrient (PhytoTechnology Laboratories, Shawnee Mission, KS 66215, USA) was administered to the TurfaceÔ along with $15 \mathrm{~mL}$ of $100 \mathrm{mM} \mathrm{NaCl}$. After these additions, the saline immersion solution was replaced so as not to dilute the concentration of the salt.

Plants grown under nitrogen limitation were administered Hoagland's nutrient solution every $3^{\text {rd }}$ day. However, this nutrient solution contained a nitrate level that was $10 \%$ that of normal Hoagland's nutrient and was prepared using no nitrogen Hoagland's Basal salt (Caisson Labs, Smithfield, UT, USA) with the addition of potassium nitrate to a level of $10 \%$ nitrogen. Bacteria Growth and Root Inoculation: HM053, a functional mutant of $A$. brasilense was obtained via a material transfer agreement between the corresponding author's institution and the Federal University of Paraná (UFPR, Curitiba, PR CEP 81531-980, Brazil). HM053 is a $\mathrm{Nif}^{+}$ constitutively expressed strain that hyper-fixes $\mathrm{N}_{2}$ excreting excess nitrogen as ammonium (Pankievicz et al., 2015). This mutant also produces high levels of auxin (Housh et al., 2021). HM053 originated as a natural mutant of the wild-type strain of $A$. brasilense FP2 (Sp7 ATCC $29145 \mathrm{Nif}^{+} \mathrm{Sm}^{\mathrm{r}} \mathrm{Nal}^{\mathrm{r}}$ ) screened through its resistance to ethylenediamine $\left(\mathrm{EDA}^{\mathrm{r}}\right)^{26,49,50}$. We grew this strain in liquid NFbHP-lactate medium following published procedures (Pankievicz et al., 
2015). Cultures were washed with sterile water and diluted to approximately $10^{6}$ to $10^{8}$ colony forming units per milliliter of culture (CFU mL $\left.{ }^{-1}\right)$. Root inoculation involved adding $1 \mathrm{~mL}$ of the inoculum to a petri dish of 10-20 maize seeds and mixing in the shaking incubator for two hours. Seeds were placed into plastic seed germination pouches (PhytoAB, Inc., San Jose, CA 95131, USA) for five days before transplanting to Turface ${ }^{\mathrm{TM}}$.

Root Phenotyping: Roots subjected to the described growth conditions were harvested from the Turface ${ }^{\mathrm{TM}}$, photographed using a DSLR camera and then weighed for fresh mass of tissue. Root photographs were processed using ImageJ freeware to determine primary root length.

Production and Administration of Radioactive $\left[{ }^{11} \mathrm{C}_{\mathrm{CO}_{2}}:\left[{ }^{11} \mathrm{C}_{\mathrm{CO}_{2}}\left(\mathrm{t}_{1 / 2}=20.4 \mathrm{~min}\right)\right.\right.$ was produced on the GE 800 Series PETtrace Cyclotron located at the Missouri Research Reactor Center using high-pressure research grade $\mathrm{N}_{2}$ gas target irradiated with a $16.4 \mathrm{MeV}$ proton beam to generate ${ }^{11} \mathrm{C}$ via the ${ }^{14} \mathrm{~N}(\mathrm{p}, \mathrm{a}){ }^{11} \mathrm{C}$ nuclear transformation ${ }^{51,52}$. The $\left[{ }^{11} \mathrm{C}\right] \mathrm{CO}_{2}$ was trapped on the molecular sieve, desorbed, and quickly released into an air stream at $200 \mathrm{~mL} \mathrm{~min}^{-1}$ as a discrete pulse for labeling a leaf affixed within a $5 \times 10 \mathrm{~cm}$ lighted $\left(560 \mathrm{mmol} \mathrm{m}^{-2} \mathrm{~s}^{-1}\right)$ leaf cell to ensure a steady level of fixation. The load leaf affixed within the cell was pulse-fed $\left[{ }^{11} \mathrm{C}^{-} \mathrm{CO}_{2}\right.$ for $1 \mathrm{~min}$, then chased with normal air for the duration of exposure. A PIN diode radiation detector (Carroll Ramsey Associates, Berkeley, CA USA) attached to the bottom of the leaf cell enabled continuous measurement of radioactivity levels within the cell during the initial pulse and in the minutes directly following to give information on $\left[{ }^{11} \mathrm{C}_{\mathrm{CO}_{2}}\right.$ fixation and leaf export of ${ }^{11} \mathrm{C}$ photosynthates ${ }^{53}$.

Whole-Plant ${ }^{11} C$-Physiology Measurements: After $\left[{ }^{11} \mathrm{C}\right] \mathrm{CO}_{2}$ pulsing, plants were incubated for 1.5 hours before separating the load leaf from shoots, roots and growth media. Roots were washed in $100 \mathrm{~mL}$ de-ionized water to remove surface-bound root exudates. Measurement of 
${ }^{11} \mathrm{C}$-activity was performed using gamma counting and data was decay corrected to end-ofbombardment. The individual components (including plant material, growth material and root wash) were summed together for total plant ${ }^{11} \mathrm{C}$-activity and normalized based on fresh tissue weight. Individual components were used to calculate leaf export, root allocation and root exudation fractions. A $10 \mathrm{~mL}$ aliquot of the root washings was processed through a $1 \mathrm{~mL}$ column packed using 200 mesh AG1 X-8 anion exchange resin (Bio-Rad Laboratories, Inc., Hercules, CA, USA). Afterwards the column and breakthrough rinse were counted for radioactivity providing a measure of the acidic and non-acidic fractions of root exudates.

$\left[{ }^{11} \mathrm{C}\right]$ Metabolite Analyses: After $\left[{ }^{11} \mathrm{C}\right] \mathrm{CO}_{2}$ pulsing and incubation for twenty minutes, leaves exposed to the radiotracer were removed and subjected to metabolite analyses following published procedures ${ }^{54}$. Tissue was flash frozen in liquid nitrogen, ground to a fine powder and extracted in methanol: water (60:40 v/v) via sonication (Branson, Bransonic 32; Sigma-Aldrich Corp. St. Louis, MO, USA) for 2 minutes at 100\% amplitude in Eppendorf ${ }^{\mathrm{TM}}$ tubes. After centrifugation at 15,000 rpm for 2 minutes the insoluble and soluble portions were separated. The soluble extract supernatant was placed in a separate Eppendorf ${ }^{\mathrm{TM}}$. A $20 \mu \mathrm{L}$ aliquot of the soluble extract and the entire insoluble pellet were counted for ${ }^{11} \mathrm{C}$-activity using a NaI gamma counter. The insoluble portion contained mostly cell-wall polymers, starch and other hydrophobic metabolites. The soluble portion contained small water-soluble compounds, including sugars, amino acids and non-nitrogen containing organic acids. All data was decay corrected back to the end of bombardment, or end of cyclotron beam.

Total soluble $\left[{ }^{11} \mathrm{C}\right]$-sugars were measured by radio thin layer chromatography (TLC) using glass backed $\mathrm{NH}_{2}$-silica HPTLC-plates $(200 \mu \mathrm{m}, \mathrm{w} / \mathrm{UV} 254)$ purchased from Sorbent Technologies (Atlanta, GA, USA) according to published procedures ${ }^{55}$. A mobile phase 
consisting of 65:20:15 acetonitrile: methanol: deionized water (v/v) was used. After development, TLC plates were imaged using autoradiography on a Typhoon 9000 imager (TyphoonTM FLA 9000, GE Healthcare, Piscataway, NJ, USA) and radioactivity was quantified using ImageQuant TL 7.0 software. Total $\left[{ }^{11} \mathrm{C}\right]$-sugar content was related to the ${ }^{11} \mathrm{C}$-activity quantified along the sample lane of the TLC plate, and then corrected to percent total fixed $\left[{ }^{11} \mathrm{C}_{\mathrm{CO}_{2}}\right.$ using gamma count data from the insoluble and soluble fractions.

$\left[{ }^{11} \mathrm{C}\right]$-Amino acids were analyzed following published procedures ${ }^{54}$ using pre-column OPA derivatization of $100 \mu \mathrm{L}$ of the methanol:water extract (1:1 ratio) and quantified by gradient radio HPLC (Sonntek, Inc. Upper Saddle River, NJ, USA). The method used a Phenomenex Gemini $5 \mu \mathrm{m} \mathrm{C} 18\left(150 \mathrm{~mm} \times 4.6 \mathrm{~mm}\right.$ inner diameter) column heated to $30{ }^{\circ} \mathrm{C}$ and mobile phase system comprised of Solvent A (95:5 0.5 M sodium acetate: methanol) and Solvent B (70:30 methanol: $18 \mathrm{M} \Omega$ water) starting at $75: 25$ and switching to $25: 75$ within 30 minutes at a flow rate of $0.7 \mathrm{~mL} \mathrm{~min}{ }^{-1}$. On-line fluorescence detection (340 $\mathrm{nm}$ excitation, $450 \mathrm{~nm}$ emission; Hitachi LaChrom Elite L-2485; Sonntek, Inc., Upper Saddle River, NJ, USA) was used for correlating retention times of standards with those of $\left[{ }^{11} \mathrm{C}\right]$-amino acids in biological samples. A NaI gamma radiation detector (Ortec, Inc., Oak Ridge, TN, USA) enabled direct measurement of radiolabeled metabolites. Data was acquired using PeakSimpleÔ chromatography software (SRI, Inc., Torrance, CA USA) where radioactivity assigned to peaks was corrected for radioactive decay, summed for a total $\left[{ }^{11} \mathrm{C}\right]$-amino acid value and related back to the amount of ${ }^{11} \mathrm{C}$ activity fixed by the plant at the start of the study.

For $\left[{ }^{11} \mathrm{C}\right]$-organic acid analysis, $500 \mu \mathrm{L}$ of leaf extract was rendered slightly basic (pH 8.5) using $1 \mathrm{~N} \mathrm{NaOH}$ and the total volume was processed through a Accell QMA Plus Light Sep$\mathrm{Pak}^{\mathrm{TM}}$ (Waters Corporation, Milford, MA, USA) followed by rinsing the contents with $10 \mathrm{~mL}$ of 
DI water. Cartridges were then counted for ${ }^{11} \mathrm{C}$ activity on a NaI gamma counter. Metabolic contents trapped on the cartridge contained small organic acids as well as amino acids. The later fraction was corrected out of the total ${ }^{11} \mathrm{C}$-activity on the cartridge by subtracting the summed, decay corrected radioactive peaks from the $\left[{ }^{11} \mathrm{C}\right]$-amino acid analysis described above.

For $\left[{ }^{11} \mathrm{C}\right]$-basic metabolite analysis, $200 \mathrm{~mL}$ of tissue extract was processed through a strong cation exchange cartridge (Strata-XL-C, Phenomenex, Inc., Torrance, CA, USA) followed by rinsing the cartridge using $10 \mathrm{~mL}$ of DI water. The cartridge was subjected to gamma counting for quantification of this metabolite pool.

Inductively Coupled Plasma-Mass Spectrometry (ICP-MS): For ICP-MS analyses of $\mathrm{K}^{+}$and $\mathrm{Ca}^{+}$ in plant tissues, roots and shoots were harvested, dried in an oven and weighed. The samples were digested in $3.0 \mathrm{~mL}$ of concentrated nitric acid at $190{ }^{\circ} \mathrm{C}$ using a Milestone Ethos Plus (Milestone SRL, Sorisole, Italy) microwave digestion system, then were diluted to $50 \mathrm{~mL}$ with ultrapure water followed by gravimetric dilution by a factor of 10 with $0.45 \mathrm{~N}$ nitric acid. Samples were analyzed via Perkin-Elmer NexION ICP-MS in Kinetic Energy Discrimination mode. Reference materials included NIST SRM 1570 spinach leaves and NIST SRM 1573 tomato leaves prepared as samples were. Internal standards at known concentrations were prepared from stock solutions (High Purity Standards, Charleston, SC 29418, USA) and used to calibrate instrument response.

Starch Analysis: Plant tissue starch levels were analyzed using Sigma-Aldrich Starch Assay Kit (Saint Louis, MO, USA) ${ }^{56}$ using enzymes to break down starch to glucose and further reactions to create a colored product for absorbance measurement at $540 \mathrm{~nm}$ using UV-vis spectrometry (Evolution 201 UV-vis spectrophotometer, Thermo Fisher Scientific, Inc., Waltham, MA, USA). Maize leaf (100-200 mg) tissue was ground using liquid nitrogen chilled $2 \mathrm{~mL}$ Eppendorf tubes 
in a ball mill grinder (Retsch Inc., Newtown, PA). Root tissue (500-1000 mg) was ground using a liquid nitrogen chilled mortar and pestle. After freeze grinding $5 \mathrm{~mL}$ of $80 \%$ aqueous ethanol was added to the root portions and $1 \mathrm{~mL}$ of this solution added to leaf portions. Samples were heated for 5 minutes at $85^{\circ} \mathrm{C}$ with constant agitation using a Cimarec hot plate (Thermo Fisher Scientific Inc., Waltham, MA, USA) equipped with an orbital shaker platform (SP Bel-Art Inc., Wayne, NJ, USA). The resulting solutions were centrifuged for 10 minutes at 1,000 x g., supernatants were removed, and pellets re-suspended in equivalent volumes of $80 \%$ aqueous ethanol before being subjected to centrifugation once more. This process removes the latent glucose residing in the plant tissues before digesting the starch to glucose.

To begin the starch digestion process, $0.2 \mathrm{~mL}$ of the $80 \%$ ethanol solution was added to each pellet. For each root and leaf sample, $2 \mathrm{~mL}$ and $1.5 \mathrm{~mL}$ of deionized (DI) water was added, respectively, and then $0.02 \mathrm{~mL}$ of $\alpha$-Amylase. Each tube was vortexed mixed and incubated for 10 minutes in boiling water bath (IKA®, C-MAG, HS10, Staufen, Germany). Samples were removed, cooled to ambient temperature, and diluted to $10 \mathrm{~mL}$ for roots and $2 \mathrm{~mL}$ for leaves using DI water. Aliquots of these diluted samples, $1 \mathrm{~mL}$ for root and $100 \mu \mathrm{L}$ for leaf samples were mixed with amyloglucosidase $(50$ units $/ \mathrm{mL})$ at a $1: 1$ ratio $(\mathrm{v} / \mathrm{v})$ and incubated in a thermomixer (Eppendorf Inc., Hamburg, Germany) at $60^{\circ} \mathrm{C}$ for 15 minutes using constant agitation. After cooling to ambient temperature, each tissue sample was again diluted in DI water (10 $\mathrm{mL}$ for roots, and $2 \mathrm{~mL}$ for leaves).

For the glucose assay, $1 \mathrm{~mL}$ of each sample was placed in a new falcon tube. Two $\mathrm{mL}$ of $o$-dianisidine, $0.1 \mathrm{mg} \mathrm{mL}^{-1}$, and reconstituted glucose oxidase/peroxidase, 12.5 units $\mathrm{mL}^{-1}$ in water was added to a sample, mixed, and placed on the hot plate. Samples were incubated for 30 minutes at $37^{\circ} \mathrm{C}$ with constant agitation. The enzymatic reaction was quenched by adding $2 \mathrm{~mL}$ 
of $6 \mathrm{M}$ sulfuric acid. The final extracts were measured using a UV Spectrophotometer at $540 \mathrm{~nm}$ (Thermo Scientific, Evolution 201, Waltham, MA, USA) and the amount of glucose derived from starch breakdown was reported as $\mathrm{mg} \mathrm{gfw}^{-1}$ tissue.

Statistical Analysis: Data was subjected to the Shapiro-Wilk Normality Test to identify outliers, so all data groups reflected normal distributions. Data was analyzed using the Student's t-test for pair-wise comparisons made between non-inoculated controls and treatment type. Statistical significance was set at $\mathrm{P}<0.05$.

\subsection{Abbreviations}

\section{PGPB Plant Growth Promoting Bacteria}




\section{References}

(1) Sarma, B. K.; Yadav, S. K.; Singh, D. P.; Singh, H. B. Rhizobacteria Mediated Induced Systemic Tolerance in Plants: Prospects for Abiotic Stress Management. In Bacteria in Agrobiology: stress management; Springer, 2012; pp 225-238.

(2) Goswami, D.; Thakker, J. N.; Dhandhukia, P. C. Portraying Mechanics of Plant Growth Promoting Rhizobacteria (PGPR): A Review. null 2016, 2 (1), 1127500.

(3) Environmental Adaptations and Stress Tolerance of Plants in the Era of Climate Change; Ahmad, P., Prasad, M. N. V., Eds.; Springer-Verlag: New York, 2012.

(4) Dwivedi, S. L.; Sahrawat, K. L.; Upadhyaya, H.; Mengoni, A.; Galardini, M.; Bazzicalupo, M.; Biondi, E. G.; Hungría, M.; Kaschuk, G.; Blair, M. W.; Ortiz, R. Advances in Host Plant and Rhizobium Genomics to Enhance Symbiotic Nitrogen Fixation InGrain Legumes. Advances in Agronomy 2015, 129, 1-116.

(5) Ilangumaran, G.; Smith, D. L. Plant Growth Promoting Rhizobacteria in Amelioration of Salinity Stress: A Systems Biology Perspective. Front. Plant Sci. 2017, 8, 1768.

(6) Zhu, J.-K. Salt and Drought Stress Signal Transduction in Plants. Annu Rev Plant Biol 2002, $53,247-273$.

(7) Hasegawa, P. M.; Bressan, R. A.; Zhu, J.-K.; Bohnert, H. J. Plant Cellular and Molecular Responses to High Salinity. Annu. Rev. Plant. Physiol. Plant. Mol. Biol. 2000, 51 (1), 463-499.

(8) Munns, R.; Tester, M. Mechanisms of Salinity Tolerance. Annual Review of Plant Biology 2008, 59 (1), 651-681.

(9) Zahedi, A. M.; Fazeli, I.; Zavareh, M.; Gerayeli, H. Evaluation of the Sensitive Components in Seedling Growth of Common Bean (Phaseolus Vulgaris L.) Affected by Salinity. Asian Journal of Crop Science 2012, 4.

(10) Nuccio, M. L.; Rhodes, D.; McNeil, S. D.; Hanson, A. D. Metabolic Engineering of Plants for Osmotic Stress Resistance. Curr Opin Plant Biol 1999, 2 (2), 128-134.

(11) Bennett, J. M.; Mutti, L. S. M.; Rao, P. S. C.; Jones, J. W. Interactive Effects of Nitrogen and Water Stresses on Biomass Accumulation, Nitrogen Uptake, and Seed Yield of Maize. Field Crops Research 1989, 19 (4), 297-311.

(12) Raun, W. R.; Johnson, G. V. Improving Nitrogen Use Efficiency for Cereal Production. Agronomy Journal 1999, 91 (3), 357-363.

(13) Hernandez, M.; Echarte, L.; Maggiora, A.; Cambareri, M.; Barbieri, P.; Cerrudo, D. Maize Water Use Efficiency and Evapotranspiration Response to N Supply under Contrasting Soil Water Availability. Field Crops Research 2015, 178.

(14) Li, Y.; Cui, S.; Zhang, Z.; Zhuang, K.; Wang, Z.; Zhang, Q. Determining Effects of Water and Nitrogen Input on Maize (Zea Mays) Yield, Water- and Nitrogen-Use Efficiency: A Global Synthesis. Sci Rep 2020, 10 (1), 9699. 
(15) Paul, M. J.; Driscoll, S. P. Sugar Repression of Photosynthesis: The Role of Carbohydrates in Signalling Nitrogen Deficiency through Source:Sink Imbalance. Plant, Cell \& Environment 1997, 20 (1), 110-116.

(16) Zhang, H.; Forde, B. G. Regulation of Arabidopsis Root Development by Nitrate Availability. $J$ Exp Bot 2000, 51 (342), 51-59.

(17) Malamy, J. E.; Ryan, K. S. Environmental Regulation of Lateral Root Initiation in Arabidopsis. Plant Physiol 2001, 127 (3), 899-909.

(18) Martin, T.; Oswald, O.; Graham, I. A. Arabidopsis Seedling Growth, Storage Lipid Mobilization, and Photosynthetic Gene Expression Are Regulated by Carbon:Nitrogen Availability. Plant Physiology 2002, 128 (2), 472-481.

(19) Malamy, J. E. Intrinsic and Environmental Response Pathways That Regulate Root System Architecture. Plant Cell Environ 2005, 28 (1), 67-77.

(20) Wingler, A.; Purdy, S.; MacLean, J. A.; Pourtau, N. The Role of Sugars in Integrating Environmental Signals during the Regulation of Leaf Senescence. J Exp Bot 2006, 57 (2), 391399.

(21) Zhang, Q. Strategies for Developing Green Super Rice. Proc Natl Acad Sci USA 2007, 104 (42), 16402.

(22) Yang, J.; Kloepper, J. W.; Ryu, C.-M. Rhizosphere Bacteria Help Plants Tolerate Abiotic Stress. Trends in Plant Science 2009, 14 (1), 1-4.

(23) Kim, Y.-C.; Glick, B. R.; Bashan, Y.; Ryu, C.-M. Enhancement of Plant Drought Tolerance by Microbes. In Plant Responses to Drought Stress; Aroca, R., Ed.; Springer Berlin Heidelberg: Berlin, Heidelberg, 2012; pp 383-413.

(24) Sarma, B. K.; Yadav, S. K.; Singh, D. P.; Singh, H. B. Rhizobacteria Mediated Induced Systemic Tolerance in Plants: Prospects for Abiotic Stress Management. In Bacteria in Agrobiology: stress management; Springer, 2012; pp 225-238.

(25) Pérez-Montaño, F.; Alías-Villegas, C.; Bellogín, R. A.; del Cerro, P.; Espuny, M. R.; Jiménez-Guerrero, I.; López-Baena, F. J.; Ollero, F. J.; Cubo, T. Plant Growth Promotion in Cereal and Leguminous Agricultural Important Plants: From Microorganism Capacities to Crop Production. Microbiological Research 2014, 169 (5-6), 325-336.

(26) Pankievicz, V. C. S.; do Amaral, F. P.; Santos, K. F. D. N.; Agtuca, B.; Xu, Y.; Schueller, M. J.; Arisi, A. C. M.; Steffens, M. B. R.; de Souza, E. M.; Pedrosa, F. O.; Stacey, G.; Ferrieri, R. A. Robust Biological Nitrogen Fixation in a Model Grass-Bacterial Association. Plant J 2015, 81 (6), 907-919.

(27) Backer, R.; Rokem, J. S.; Ilangumaran, G.; Lamont, J.; Praslickova, D.; Ricci, E.;

Subramanian, S.; Smith, D. L. Plant Growth-Promoting Rhizobacteria: Context, Mechanisms of Action, and Roadmap to Commercialization of Biostimulants for Sustainable Agriculture. Front. Plant Sci. 2018, 9.

(28) Reinhold-Hurek, B.; Hurek, T. Life in Grasses: Diazotrophic Endophytes. Trends in Microbiology 1998, 6 (4), 139-144. 
(29) Reinhold-Hurek, B.; Hurek, T. Living inside Plants: Bacterial Endophytes. Current Opinion in Plant Biology 2011, 14 (4), 435-443.

(30) do Amaral, F. P.; Agtuca, B. J.; Stacey, G. Setaria Root-Microbe Interactions. In Genetics and Genomics of Setaria; Doust, A., Diao, X., Eds.; Plant Genetics and Genomics: Crops and Models; Springer International Publishing: Cham, 2017; Vol. 19, pp 239-250.

(31) Kloepper, J. W. Plant Growth Promoting Rhizobacteria as Biological Control Agents. In Soil microbial ecology-applications in agricultural and environmental management.; Marcel Dekker: New York, NY, 1993; pp 255-274.

(32) van Loon, L. C. Plant Responses to Plant Growth-Promoting Rhizobacteria. In New Perspectives and Approaches in Plant Growth-Promoting Rhizobacteria Research; Bakker, P. A. H. M., Raaijmakers, J. M., Bloemberg, G., Höfte, M., Lemanceau, P., Cooke, B. M., Eds.; Springer Netherlands: Dordrecht, 2007; pp 243-254.

(33) Jin, C. W.; Ye, Y. Q.; Zheng, S. J. An Underground Tale: Contribution of Microbial Activity to Plant Iron Acquisition via Ecological Processes. Annals of Botany 2014, 113 (1), 7 18 .

(34) Kuan, K. B.; Othman, R.; Abdul Rahim, K.; Shamsuddin, Z. H. Plant Growth-Promoting Rhizobacteria Inoculation to Enhance Vegetative Growth, Nitrogen Fixation and Nitrogen Remobilisation of Maize under Greenhouse Conditions. PLoS ONE 2016, 11 (3), e0152478.

(35) Ryu, C.-M.; Farag, M. A.; Hu, C.-H.; Reddy, M. S.; Kloepper, J. W.; Paré, P. W. Bacterial Volatiles Induce Systemic Resistance in Arabidopsis. Plant Physiol 2004, 134 (3), 1017-1026.

(36) Wenke, K.; Weise, T.; Warnke, R.; Valverde, C.; Wanke, D.; Kai, M.; Piechulla, B. Bacterial Volatiles Mediating Information Between Bacteria and Plants. In Biocommunication of Plants; Witzany, G., Baluška, F., Eds.; Signaling and Communication in Plants; Springer Berlin Heidelberg: Berlin, Heidelberg, 2012; Vol. 14, pp 327-347.

(37) Santoro, M. V.; Bogino, P. C.; Nocelli, N.; Cappellari, L. del R.; Giordano, W. F.; Banchio, E. Analysis of Plant Growth-Promoting Effects of Fluorescent Pseudomonas Strains Isolated from Mentha Piperita Rhizosphere and Effects of Their Volatile Organic Compounds on Essential Oil Composition. Front. Microbiol. 2016, 7.

(38) Cappellari, L. del R.; Banchio, E. Microbial Volatile Organic Compounds Produced by Bacillus Amyloliquefaciens GB03 Ameliorate the Effects of Salt Stress in Mentha Piperita Principally Through Acetoin Emission. Journal of Plant Growth Regulation 2020, 39 (2), 764 775.

(39) Dobereiner, J.; Pedrosa, F. O. Nitrogen-Fixing Bacteria in Nonleguminous Crop Plants.; Science Tech, Springer Verlag: Madison, WI, 1987; Vol. 63.

(40) Creus, C.; Sueldo, R.; Barassi, C. Water Relations and Yield in Azospirillum-Inoculated Wheat Exposed to Drought in the Field. 2004.

(41) Barassi, C. A.; Ayrault, G.; Creus, C. M.; Sueldo, R. J.; Sobrero, M. T. Seed Inoculation with Azospirillum Mitigates $\mathrm{NaCl}$ Effects on Lettuce. Scientia Horticulturae 2006, 109 (1), 8 14. 
(42) Rodríguez-Salazar, J.; Suárez, R.; Caballero-Mellado, J.; Iturriaga, G. Trehalose Accumulation in Azospirillum Brasilense Improves Drought Tolerance and Biomass in Maize Plants. FEMS Microbiol Lett 2009, 296 (1), 52-59.

(43) Carrozzi, L. E.; Creus, C. M.; Barassi, C. A.; Monterubbianesi, G.; Di Benedetto, A. Reparation of Aged Lettuce ( Lactuca Sativa ) Seeds by Osmotic Priming and Azospirillum Brasilense Inoculation. Botany 2012, 90 (11), 1093-1102.

(44) Fasciglione, G.; Casanovas, E.; Quillehauquy, V.; Yommi, A.; Goñi, M.; Roura, S.; Barassi, C. Azospirillum Inoculation Effects on Growth, Product Quality and Storage Life of Lettuce Plants Grown under Salt Stress. Scientia Horticulturae 2015, 195, 154-162.

(45) Scott, S.; Housh, A.; Powell, G.; Anstaett, A.; Gerheart, A.; Benoit, M.; Wilder, S.; Schueller, M.; Ferrieri, R. Crop Yield, Ferritin and Fe(II) Boosted by Azospirillum Brasilense (HM053) in Corn. Agronomy 2020, 10 (3), 394.

(46) Pankievicz, V. C. S.; do Amaral, F. P.; Santos, K. F. D. N.; Agtuca, B.; Xu, Y.; Schueller, M. J.; Arisi, A. C. M.; Steffens, M. B. R.; de Souza, E. M.; Pedrosa, F. O.; Stacey, G.; Ferrieri, R. A. Robust Biological Nitrogen Fixation in a Model Grass-Bacterial Association. Plant J 2015, 81 (6), 907-919.

(47) Housh, A. B.; Powell, G.; Scott, S.; Anstaett, A.; Gerheart, A.; Benoit, M.; Waller, S.; Powell, A.; Guthrie, J. M.; Higgins, B.; Wilder, S. L.; Schueller, M. J.; Ferrieri, R. A. Functional Mutants of Azospirillum Brasilense Elicit Beneficial Physiological and Metabolic Responses in Zea Mays Contributing to Increased Host Iron Assimilation. The ISME Journal 2021, 1-18.

(48) Housh, A.; Powell, G.; Anstaett, A.; Gerheart, A.; Benoit, M.; Powell, A.; Waller, S.; Guthrie, J.; Higgins, B.; Wilder, S.; Schueller, M.; Ferrieri, R. A. Functional Mutants of Azospirillum Brasilense Bacterium Elicit Beneficial Physiological and Metabolic Responses in Zea Mays That Contribute to Increased Host Iron Assimilation. ISME Journal accepted, in revision.

(49) Machado, H. B.; Funayama, S.; Rigo, L. U.; Pedrosa, F. O. Excretion of Ammonium by Azospirillum Brasilense Mutants Resistant to Ethylenediamine. Can. J. Microbiol. 1991, 37 (7), 549-553.

(50) Santos, A. R. S.; Etto, R. M.; Furmam, R. W.; Freitas, D. L. de; Santos, K. F. d'Eça N.; Souza, E. M. de; Pedrosa, F. de O.; Ayub, R. A.; Steffens, M. B. R.; Galvão, C. W. Labeled Azospirillum Brasilense Wild Type and Excretion-Ammonium Strains in Association with Barley Roots. Plant Physiology and Biochemistry 2017, 118, 422-426.

(51) Ferrieri, R. A.; Wolf, A. P. The Chemistry of Positron Emitting Nucleogenic (Hot) Atoms with Regard to Preparation of Labelled Compounds of Practical Utility. Radiochimica Acta 1983, $34(1 / 2), 69-83$.

(52) Ferrieri, R. A. Handbook of Radiopharmaceuticals: Radiochemistry and Applications. In Production and application of synthetic precursors labeled with carbon-11 and fluorine-18; John Wiley and Sons, Ltd., 2003.

(53) Ferrieri, R. A.; Gray, D. W.; Babst, B. A.; Schueller, M. J.; Schlyer, D. J.; Thorpe, M. R.; Orians, C. M.; Lerdau, M. Use of Carbon-11 in Populus Shows That Exogenous Jasmonic Acid 
Increases Biosynthesis of Isoprene from Recently Fixed Carbon. Plant, Cell \& Environment 2005, 28 (5), 591-602.

(54) Qu, W.; Robert, C. A. M.; Erb, M.; Hibbard, B. E.; Paven, M.; Gleede, T.; Riehl, B.; Kersting, L.; Cankaya, A. S.; Kunert, A. T.; Xu, Y.; Schueller, M. J.; Shea, C.; Alexoff, D.; Lee, S. J.; Fowler, J. S.; Ferrieri, R. A. Dynamic Precision Phenotyping Reveals Mechanism of Crop Tolerance to Root Herbivory[OPEN]. Plant Physiol 2016, 172 (2), 776-788.

(55) Babst, B. A.; Karve, A. A.; Judt, T. Radio-Metabolite Analysis of Carbon-11 Biochemical Partitioning to Non-Structural Carbohydrates for Integrated Metabolism and Transport Studies. Plant Cell Physiol. 2013, 54 (6), 1016-1025.

(56) Sigma-Aldrich Technical Bulletin - Starch Assay Kit Amylase/Amyloglucosidase Method. Sigma-Aldrich 2019.

(57) Nahar, K.; Hasanuzzaman, M.; Fujita, M. Roles of Osmolytes in Plant Adaptation to Drought and Salinity. In Osmolytes and Plants Acclimation to Changing Environment: Emerging Omics Technologies; Iqbal, N., Nazar, R., A. Khan, N., Eds.; Springer India: New Delhi, 2016; pp 37-68.

(58) Seifikalhor, M.; Aliniaeifard, S.; Shomali, A.; Azad, N.; Hassani, B.; Lastochkina, O.; Li, T. Calcium Signaling and Salt Tolerance Are Diversely Entwined in Plants. Plant Signaling \& Behavior 2019, 14 (11), 1665455.

(59) Turan, M. A.; Elkarim, A. H. A.; Nilguuml; Taban, N.; Taban, S. Effect of Salt Stress on Growth and Ion Distribution and Accumulation in Shoot and Root of Maize Plant. AJAR 2010, 5 (7), 584-588.

(60) Ilangumaran, G.; Smith, D. L. Plant Growth Promoting Rhizobacteria in Amelioration of Salinity Stress: A Systems Biology Perspective. Front. Plant Sci. 2017, 8.

(61) Munns, R.; Tester, M. Mechanisms of Salinity Tolerance. Annual Review of Plant Biology 2008, 59 (1), 651-681.

(62) Jung, J. K. H. M.; McCouch, S. R. M. Getting to the Roots of It: Genetic and Hormonal Control of Root Architecture. Front. Plant Sci. 2013, 4.

(63) Rahnama, A.; Munns, R.; Poustini, K.; Watt, M. A Screening Method to Identify Genetic Variation in Root Growth Response to a Salinity Gradient. J Exp Bot 2011, 62 (1), 69-77.

(64) Wu, J.; Hettenhausen, C.; Meldau, S.; Baldwin, I. T. Herbivory Rapidly Activates MAPK Signaling in Attacked and Unattacked Leaf Regions but Not between Leaves of Nicotiana Attenuata. Plant Cell 2007, 19 (3), 1096-1122.

(65) Campanelli, A.; Ruta, C.; De Mastro, G.; Morone-Fortunato, I. The Role of Arbuscular Mycorrhizal Fungi in Alleviating Salt Stress in Medicago Sativa L. Var. Icon. Symbiosis 2012, 59.

(66) Alqarawi, A. A.; Allah, E. F. A.; Hashem, A. Alleviation of Salt-Induced Adverse Impact via Mycorrhizal Fungi in Ephedra Aphylla Forssk. Journal of Plant Interactions 2014, 9 (1), 802-810. 
(67) Cappellari, L.; Banchio, E. Microbial Volatile Organic Compounds Produced by Bacillus Amyloliquefaciens GB03 Ameliorate the Effects of Salt Stress in Mentha Piperita Principally Through Acetoin Emission. Journal of Plant Growth Regulation 2020, 39.

(68) Kapoor, R.; Sharma, D.; Bhatnagar, A. K. Arbuscular Mycorrhizae in Micropropagation Systems and Their Potential Applications. Scientia Horticulturae 2008, 116 (3), 227-239.

(69) Evelin, H.; Devi, T. S.; Gupta, S.; Kapoor, R. Mitigation of Salinity Stress in Plants by Arbuscular Mycorrhizal Symbiosis: Current Understanding and New Challenges. Front. Plant Sci. 2019, 10.

(70) López-Bucio, J.; Cruz-Ramírez, A.; Herrera-Estrella, L. The Role of Nutrient Availability in Regulating Root Architecture. Curr Opin Plant Biol 2003, 6 (3), 280-287.

(71) Callahan, H. S.; Pigliucci, M.; Schlichting, C. D. Developmental Phenotypic Plasticity: Where Ecology and Evolution Meet Molecular Biology. Bioessays 1997, 19 (6), 519-525.

(72) Kalauni, S.; Shrestha, R. K.; Ojha, R. B.; Santosh, K. C. Nitrogen Stress on Maize Roots in Subtropical Condition of Nepal. Int J Soil Sci 2016, 11 (4), 137-142.

(73) Zhang, Y.; Liu, Z.; Liu, J.; Lin, S.; Wang, J.; Lin, W.; Xu, W. GA-DELLA Pathway Is Involved in Regulation of Nitrogen Deficiency-Induced Anthocyanin Accumulation. Plant Cell Rep 2017, 36 (4), 557-569.

(74) Loreti, E.; Povero, G.; Novi, G.; Solfanelli, C.; Alpi, A.; Perata, P. Gibberellins, Jasmonate and Abscisic Acid Modulate the Sucrose-Induced Expression of Anthocyanin Biosynthetic Genes in Arabidopsis. New Phytol 2008, 179 (4), 1004-1016.

(75) Teng, S.; Keurentjes, J.; Bentsink, L.; Koornneef, M.; Smeekens, S. Sucrose-Specific Induction of Anthocyanin Biosynthesis in Arabidopsis Requires the MYB75/PAP1 Gene. Plant Physiology 2005, 139 (4), 1840-1852.

(76) Pankievicz, V. C. S.; Irving, T. B.; Maia, L. G. S.; Ané, J.-M. Are We There yet? The Long Walk towards the Development of Efficient Symbiotic Associations between Nitrogen-Fixing Bacteria and Non-Leguminous Crops. BMC Biology 2019, 17 (1), 99.

(77) Ferrieri, R. A.; Herman, E.; Babst, B.; Schueller, M. J.; Herman, E.; Babst, B.; Schueller, M. J. Managing the Soil Nitrogen Cycle in Agroecosystems https:/www.taylorfrancis.com/ (accessed May 6, 2020). 


\section{Chapter Six: A Guiding Light to Sustaining Life Functions Belowground: Light effects on biological nitrogen fixation, carbon dioxide assimilation, and iron uptake in functional mutants of Azospirillum brasilense.}

\subsection{Introduction}

Light has been a vital driver for life on Earth, but it has also proved to be threatening to aerobic organisms due to the photodynamic effect ${ }^{1}$. Over time, organisms developed adaptations to cope with, or even thrive off, light. It is common knowledge that, as autotrophs, light has vital importance to the growth and development of plants. What is less known is that light also impacts growth and development of plant roots below ground ${ }^{2}$. Indirectly, light can affect root processes when signaling chemicals from the shoot cells triggered by light travel to the root cells and elicit a response. Only a few signaling chemicals have been identified as being directly linked to light responses, which include photosynthetic sugars, plant hormones, and the transcription factor, $\mathrm{HY}^{3}$.

Of the sugars, sucrose was shown to be vital to plant root growth in Arabidopsis $^{4}$. When photosynthesis was blocked in seedlings the plant roots would not grow, but the addition of sucrose to the growth media allowed a rescue of the root growth ${ }^{4}$. The plant hormones associated with light signaling are auxin and gibberellic acid $(\mathrm{GA})^{3}$. A large amount of auxin is produced in the young leaf tissues of plants and transported to the roots where it influences root development. Photoreceptors sensitive to red and far-red light (PHYs) are inactivated in the presence of light, which triggers the biosynthesis of auxin in the shoot. It is then trafficked rootward by PIN proteins PIN1 and PIN2 while exposed to light, meanwhile in darkness the transport of auxin by PIN1 is greatly reduced ${ }^{3}$. GA is involved in many growth and developmental processes; one of which, observed in Arabidopsis ${ }^{5}$, is root growth. Red and far-red light have been seen to elevate 
expression of the GA20OX genes in Arabidopsis, responsible for GA biosynthesis of which there are various forms of GA only some of which are biologically active. Under these light conditions, $\mathrm{GA}^{12}$, which is not bioactive, is trafficked to the roots where it is converted to a biologically active form by GA20ox and GA3ox enzymes where it begins to stimulate root growth $^{5}$. HY5 is a transcription factor involved in light, hormone, and stress signaling ${ }^{3}$. It has been shown to be transported in the presence of light from shoot to root tissues ${ }^{6}$, where it activates its own expression in the root along with $\mathrm{HYH}$ expression. Both of these transcription factors regulate lateral root growth, development, and root gravitropism responses ${ }^{3}$.

Roots themselves also have photoreceptors, which can be directly triggered by light if light can make it to these underground tissues ${ }^{2}$. In an experiment published in Science Signaling in 2016 by Hyo-Jun Lee, et al., it was demonstrated that roots in Arabidopsis thaliana directly perceived certain wavelengths of light (predominantly red light) conducted, or "piped", from shoot to root tissues ${ }^{2}$. This light transmission occurs along the internal tissues of plants more readily in the longer wavelengths such as red and far-red light than it does for the shorter wavelengths such as blue or green ${ }^{3}$. The light is first, and predominantly, piped in the stem tissue where it can activate root-expressed phyB, which then regulates HY5. The HY5 transcription factor has a role in root growth in response to light, moderating root gravitropism responses ${ }^{2}$ and nitrogen uptake $\mathrm{s}^{3}$. Root phototropism is not the same as shoot phototropism- in flowering plants roots grow away from blue and white lights, but toward red light ${ }^{7}$. To date, light-piping has also been described in bean plants (Phaseolus vulgaris) ${ }^{8}$. This light-piping is expected to have a modest impact on roots, in terms of direct light signaling, because conductance has been shown to decrease to $1 \%$ after $3 \mathrm{~cm}$ of traveled distance inside the plant in herbaceous plants ${ }^{3}$. These modest impacts include HY5 stimulation of nitrate uptake in the roots via the NRT2;1 transporter 
to keep $\mathrm{N}$ and $\mathrm{C}$ demands balanced in the plant ${ }^{6}$ as well as a reduction in the length of the primary root and lateral roots in the absence of red and far-red light sensing in the roots ${ }^{9}$. It is thought that the reduction in root length helps to ensure more of the plant's resources go to light capture $^{3}$. If plant roots contain photoreceptors and are able to receive light stimuli through conduction from the aerial portions of the plant, it becomes of interest whether plant growth promoting bacteria (PGPB), which associate with plant roots, can also sense and respond to the same light stimulus.

Bacteria of the genus Azospirillum are Gram-negative, nitrogen(N)-fixing bacteria found in the rhizosphere, which are known for their plant growth promoting effects on grasses and cereals worldwide. ${ }^{10-12}$ Other diazotrophs associated with plant roots include Acetobacter diazotrophicus, Herbaspirillum seropedicae, Azoarcus spp., and Azotobacter. Azospirillum brasilense are predominantly root surface colonizing, or epiphytic, while species such as $A$. diazotrophicus, H. seropedicae, and Azoarcus spp. are endophytic. ${ }^{12}$ When soil and environmental conditions are ideal, Azospirillum can promote plant growth, yield, and overall $\mathrm{N}$-content ${ }^{13}$. These PGPB are thought to positively impact plant performance due to mechanisms such as their biological nitrogen fixation ${ }^{14}$ and plant growth hormone (i.e., auxin) production capabilities $^{12}$. The positive impacts this bacteria has on its host plants has led to the commercialization and use of these bacteria inoculants in certain areas of the world ${ }^{15-17}$.

Bacteria can be sensitive to light, most notably the photosynthetic species such as cyanobacteria, with the most well-studied photoreceptor in photosynthetic bacteria being a phytochrome called bacteriophytochrome ${ }^{18}$. Photoreceptors are not only present in photosynthetic bacteria. Phytochromes are a family of red/far-red responsive photoreceptors present in both photosynthetic and non-photosynthetic organisms, in which the chromophore 
photo transforms between the $\mathrm{E}$ and $\mathrm{Z}$ conformations based on exposure to red or far-red light wavelengths ${ }^{1}$. Azospirillum brasilense contains genes for bacertiophytochromes that control carotenoid-independent responses to photodynamic stress ${ }^{1}$. This indicates that although these bacteria are not photosynthetic, they are equipped to sense light and respond to it. Currently, there is not much understood about microbial light-sensing and the impact of this stimuli on the biological functions of $A$. brasilense belowground.

A. brasilense presents a wide range of carbon metabolism and can sustain growth on many forms of carbon such as various sugars and organic acids to include D-gluconate, Dfructose, ketoglutarate, pyruvate, and D-Lactate ${ }^{19-21}$. It has also been demonstrated that different functional mutants of $A$. brasilense are capable of sequestering differing amounts of $\mathrm{CO}_{2}$ from the atmosphere that correlates with their $\mathrm{N}_{2}$-fixing capacity ${ }^{22}$. In prokaryotes, carbonic anhydrases (CAs) are involved in diverse biochemical and physiological processes, including photosynthesis, respiration, $\mathrm{CO}_{2}$ and ion transport, and $\mathrm{CO}_{2} /$ bicarbonate balance required for biosynthetic reactions ${ }^{23}$. Although the wealth of information known about the function of this enzyme in prokaryotes relates to its role in the fixation of $\mathrm{CO}_{2}$ by phototrophic microbes, relatively little is known about the physiological roles of CAs in non-photosynthetic organisms. However, in acetogenic bacteria ${ }^{23}$ and methanogenic archaea ${ }^{24}$, both appear to utilize significant amounts of sequestered $\mathrm{CO}_{2}$ in central metabolic processes yielding essential amino acids, nucleotides, and fatty acids. Like these examples, A. brasilense is not a photosynthetic organism, but does possess CAs and phosphoenol pyruvate carboxylase PEP-C, enabling efficient sequestration and metabolism of $\mathrm{CO}_{2}{ }^{25}$. This suggests it is likely that the functional mutants investigated in this work will assimilate and perhaps show differences in metabolism under differing treatment conditions. 
The biological nitrogen fixation (BNF) ability of Azospirillum brasilense has been of key interest in this PGPB across the literature. BNF is a multi-electron redox process carried out by the nitrogenase enzyme in diazotrophic species and converts atmospheric nitrogen $\left(\mathrm{N}_{2}\right)$ to a biologically usable form, $\mathrm{NH}_{3}{ }^{26}$. The structural genes (nif) for all BNF bacteria are highly conserved and NifA, the transcriptional activator, is required for other nif genes to be expressed. ${ }^{12}$ Nitrogenase is a two-protein enzyme and these two proteins are iron $(\mathrm{Fe})$ containing; dinitrogenase, which contains iron and molybdenum, and dinitrogenase reductase, contains iron. ${ }^{26}$ The iron protein is the only known electron donor to support the nitrogen fixation process, thus is vital for its function. The predominant oxidation state of the iron in the nitrogenase enzyme is $\mathrm{Fe}^{2+26}$.

In $A$. brasilense, transcriptional regulation of the NIFA gene is turned "on" at all times and is down regulated by the $\mathrm{P}_{\pi}$ protein in the presence of specific environmental stimuli, such as excess $\mathrm{NH}_{4}{ }^{+}$concentrations ${ }^{27}$. Environmental factors can exert effects on nitrogenase in $\mathrm{A}$. brasilense. Nitrogenase loses activity in excess $\mathrm{NH}_{4}{ }^{+}$and high- $\mathrm{O}_{2}$ environments ${ }^{27}$. In addition, nitrate and nitrite have the same immediate effects on nitrogenase as observed with $\mathrm{NH}_{4}{ }^{+28}$. Other soil bacteria species, like Rhodospirillum rubrum, can have nitrogenase functions sensitive to light ${ }^{29}$, although this bacteria is photosynthetic and this sensitivity has not yet been explored for A. brasilense.

Much known about PGPB, A. brasilense specifically, is favorable in terms of an agricultural enhancement strategy, but there is much about the mechanisms of action and robustness of the application to the field that is unknown. In this work, we set out to better understand the metabolism and physiology of functional mutants of the common PGPB, Azospirillum brasilense, in terms of iron needs and uptake, nitrogenase activity, and carbon 
assimilation and further metabolism as a function of presence of light. To our knowledge, this is the first time a light sensitivity has been demonstrated in biological functions of $A$. brasilense.

\subsection{Results and Discussion}

Light piping in Zea mays- The

phenomenon of light piping has not

yet been shown to occur in maize. In

order to investigate whether light

conduction could occur, and thus

influence functional mutants of $A$.

brasilense in association with maize,

we set up a young maize plant in a

black box, exposed the shoots to

light, and took images of a cut root

with an infrared modified camera

(Figure 6.2.1.). The upper stem of a

maize plant was cut and inserted into

a black light tight plastic tube lined

with aluminum foil. This helped

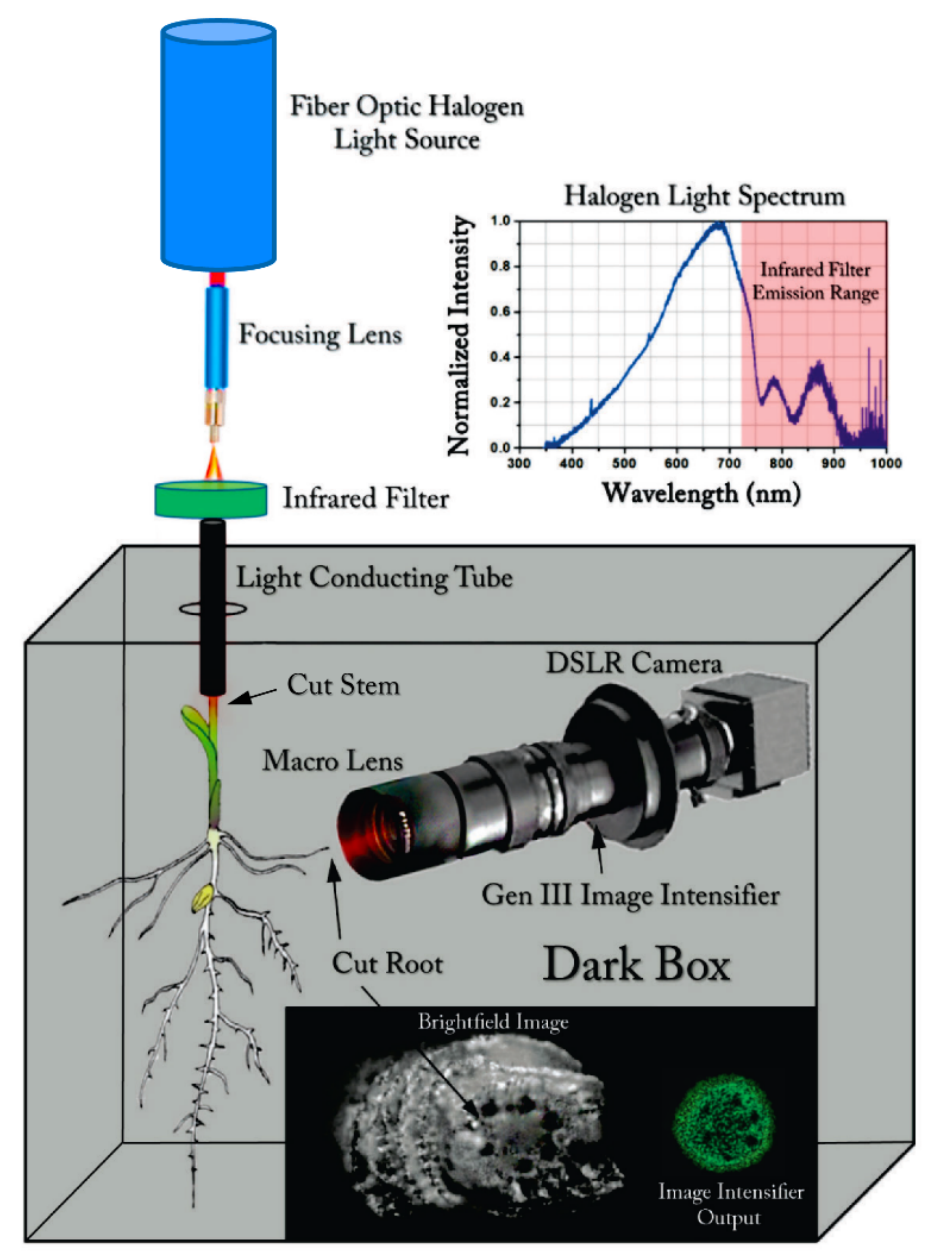

Figure 6.2.1. Light Piping Setup and Evidence of Light Piping in Maize. This data was contributed conduct light from a fiber optic by Spenser Waller, U. of Missouri Dept. of Natural Resources.

halogen light source through an infrared filter, which cut out all visible light below $720 \mathrm{~nm}$, to the cut stem end. The plant stem was affixed to a dark box using plumbers putty making for a light tight seal. A single root, one of the crown roots due to its larger size, was chosen for examination and the end of the root was cleanly cut with a razor blade. That cut root end was 
mounted facing a Canon 1:5 macro lens brought to focus by allowing some ambient light to leak into the dark box - that produced the brightfield root end image (Figure 6.2.1.). The box was then completely sealed with light tight tape and the halogen light was turned on. The shutter exposure setting was 300 seconds with the camera's digital iso gain setting for the sensor adjusted to 100 . IR light conducted through the stem and roots was sensed and recorded by the optical system.

A previous publication by Sun et al. in 2004 investigated light conductance in the stem and root of various herbaceous plants, one being Zea mays, and they noted light conduction in the root did occur at high levels of far-red and nearly infra-red light ${ }^{30}$. In their results, light was predominantly conducted in the living fibres and vessels associated with xylem and phloem most efficiently, but also was efficient via the lumina cells of the pith and cortex. When a comparison is made between the bright field image and the image intensifier output in our study (Figure 6.2.1.), the inner endodermal ring was seen to light up more than the outside cortical zone or epidermal edges of the cut root. This agrees with published observations that the epidermis is a poor light conductor. The darker xylem inner circles are not lit up with IR light, but it is the living cells making up the outer rings of the xylem and phloem, which should conduct light. It is not totally clear from our images, due to the resolution and lack of staining, whether these tissues conducted light well, however the greatest conductance in our image is observed in the endodermal ring area of the root, which is near where many vascular tissues are also found. The pith and cortex in our image appear to conduct light as well, in agreeance with the Sun et al. publication. This image demonstrates that red to far-red light focused into the shoot tissue was transmitted along the cell walls and made it to the root aligning well with previously published observations in light piping patterns in maize ${ }^{30}$. 
Acetylene Reduction Assays (ARAs) of Functional

Mutants of A. brasilense under Various Light

Conditions - To investigate whether the function of

the nitrogenase enzyme, responsible for BNF

capacity, is impacted by light stimulus an ARA was

performed with maize roots inoculated with these

functional mutant strains of bacteria in both white

light and dark conditions (Figure 6.2.2.) as well as

red and blue light conditions (Figure 6.2.3.). In the

white light and dark ARA studies, it was noted that

the ethylene levels were observed to slowly

increase over root incubation time with acetylene.

This is expected based on natural root emissions of

their own ethylene while incubating in the closed

jar, as measured on non-inoculated maize roots ${ }^{31}$

and shown in Chapter 3 Figure 3.3.7. on page 64.

Since the ethylene values were normalized for root

mass, this does not significantly impact the data as

it appears in the figure.

What is clear from the light/dark ARA

studies is that the BNF bacteria mutants, HM053

and $i p d C$, show a significant light response in terms of

ethylene production- indicating that the nitrogenase
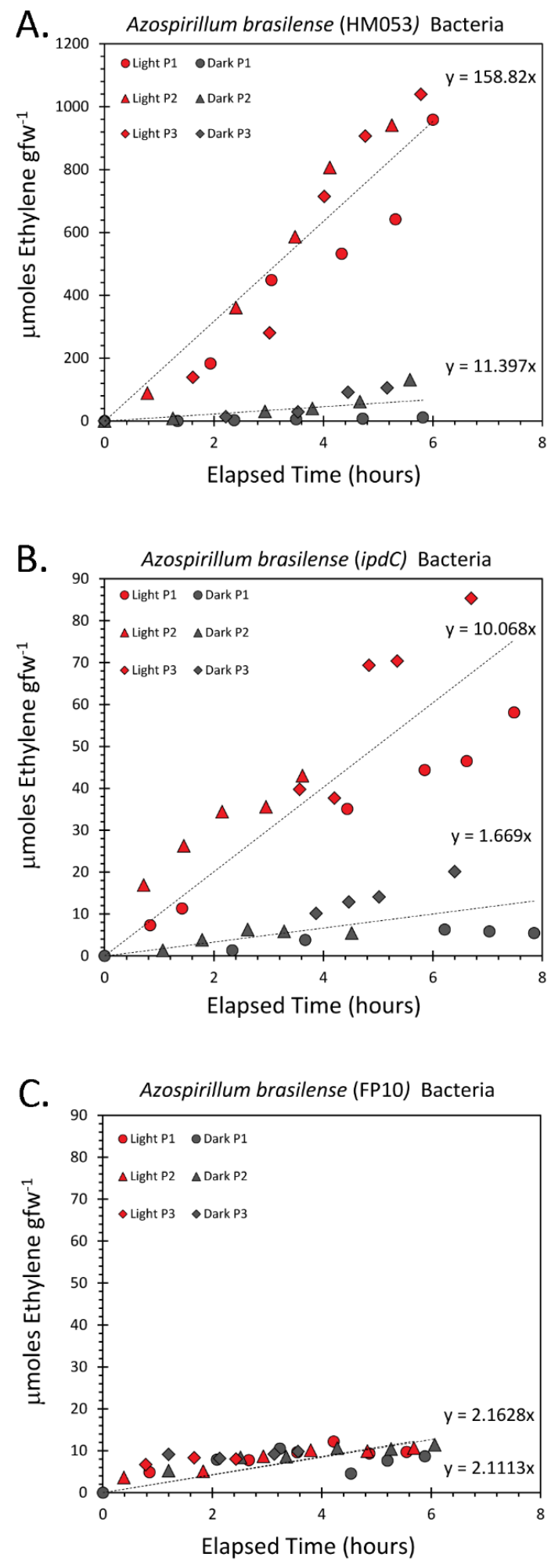

Figure 6.2.2.- ARA of $A$. brasilense inoculated maize roots as a function of white light and total darkness. 
enzyme active in both of these bacteria is more active in the presence of light than they are in the dark (Figure 6.2.2. A and B). HM053, which fixes much more nitrogen than $i p d C$, has values much greater than observed in the latter especially while exposed to light, producing upwards of $1000 \mu \mathrm{mol}$ ethylene (gfw of root) $)^{-1}$ after 6 hours compared to the $90 \mu \mathrm{mol}$ ethylene (gfw of root) ${ }^{-1}$ respectively. Strikingly, the FP10 mutant, which does not contain an active nitrogenase gene, does not exhibit any changes in ethylene production when exposed to light vs dark conditions (Figure 6.2.2. C). This seems to point to light sensitivity of the nitrogenase enzyme, or at least the BNF capacity of the bacteria, in A. brasilense. Light sensitivity in nitrogenase function has been observed in oceanic photosynthetic bacteria, Crocosphaera watsonii, in which exposure of the bacteria to high intensities of blue and amber light (over 700 $\mu \mathrm{mol} \mathrm{m} \mathrm{m}^{-2} \mathrm{~s}^{-1}$ ) exhibited decreases in ethylene production as noted by ARA ${ }^{32}$. In another photosynthetic bacteria species, Rhodopseudomonas palustris, a nitrogenase variant exists in which two amino acid substitutions were observed in the FeMo protein allowing the enzyme to reduce $\mathrm{CO}_{2}$ to $\mathrm{CH}_{4}{ }^{33}$. In these bacteria, the nitrogenase-variant enzyme was seen to produce methane only in the presence of light and more methane as the light intensity increased with the limitation that the highest intensity explored was $60 \mu \mathrm{mol} \mathrm{m} \mathrm{m}^{-2} \mathrm{~s}^{-133}$. While light impacts on nitrogenase in non-photosynthetic bacteria have not been explored, and impacts for photosynthetic bacteria have not been deeply explored, light clearly is shown in these instances to have an impact on the BNF capacity. A. brasilense functional mutants did not exhibit any BNF inhibition with exposure to light, as noted for Crocosphaera watsonii, and our studies were conducted at relatable intensities for the white light vs dark studies, and much higher intensities for the red/blue ARA studies. This indicates that light influences on BNF activity are likely not conserved between photosynthetic and non-photosynthetic bacteria species. 

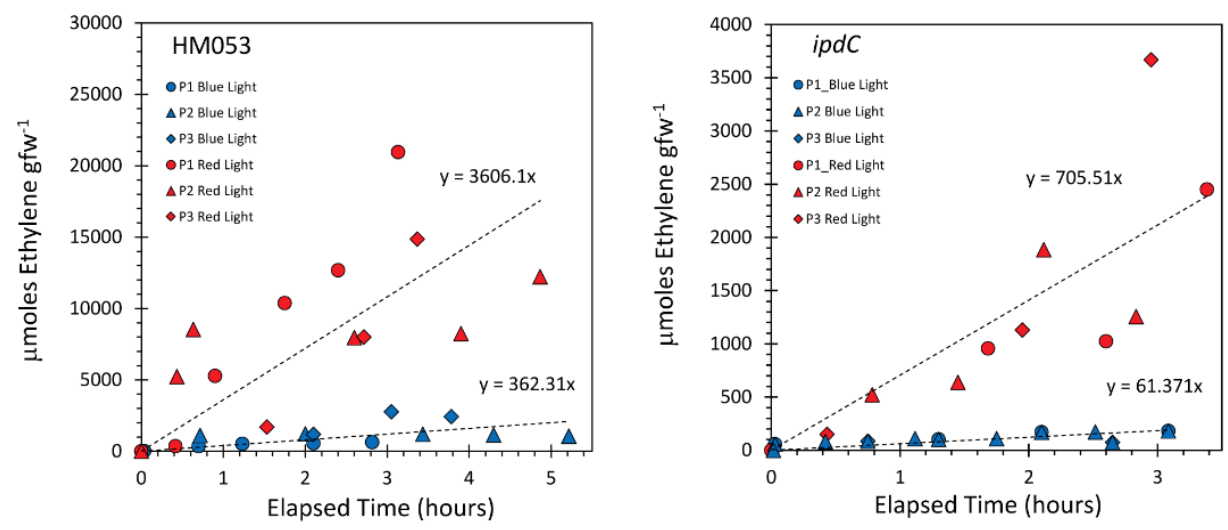

The elevated

activity of the

nitrogenase enzyme

in the BNF active

bacteria functional

mutants (HM053

Figure 6.2.3. ARA of $A$. brasilense inoculated maize roots as a function of blue and red light exposure.

and $i p d C)$

corresponds to elevated ferric radiotracer uptake in both bacteria as well. Indeed, the ferric

radiotracer uptake is highest where the nitrogenase activity is greatest, with HM053, which could indicate the use of some excess ferric iron for nitrogenase and related activities. Upon noting a light sensitivity in nitrogenase activity as measured by ARA in the bacteria mutants with BNF capacity, a deeper look at the wavelength sensitivity was completed, in an abbreviated study similar to an action spectrum, by exposing both HM053 and ipdC inoculated maize roots to blue and red light and measuring ethylene production over time (Figure 6.2.3.). HM053 and ipdC were selected because in the white light and darkness ARAs, they were the only two that demonstrated a light response. As shown in Figure 6.2.3. both functional mutants are more responsive, showing more ethylene produced by the nitrogenase enzyme, to the red light than blue. It is notable that, just as in the white light and darkness ARA studies, HM053 shows more elevated acetylene turnover to ethylene by nitrogenase than $i p d C$, which is expected due to the constitutive expression of its NIF gene. The greater response in the red light over the blue for both mutants suggests BNF is not only responsive to light, but that it is more responsive to red wavelengths than blue wavelengths. While it may appear that these bacteria are unresponsive to the blue light, it should be noted the slope of the response over time, for HM053, is 362 when 
exposed to the blue light compared to 11.3 in darkness. Thus, HM053 responds to light across the entire electromagnetic spectrum but favors red light - at least in regulating nitrogenase It is known that Azospirillum brasilense has bacteriophytochrome genes sensitive to red and far-red light ${ }^{1}$, and that plants are capable of transmitting light, mostly red light, from the shoot to root tissue $^{3}$. It is possible then that light piping by the host plant stimulates BNF function in $A$. brasilense.

There is a large difference in magnitude in acetylene-to-ethylene conversion between the red and blue light studies relative to the white light and darkness studies. This is probably due to the 5-fold increase in light intensity compared to the white light panel (red/blue LED lights had an intensity of $2800 \mathrm{mmole}^{-2} \mathrm{~s}^{-1}$ compared to the $550 \mathrm{mmole} \mathrm{m}^{-2} \mathrm{~s}^{-1}$ for white LED light panel) and perhaps higher temperature $\left(39.8^{\circ} \mathrm{C}\right.$ during sample collection for the red/blue light studies vs. $30.2^{\circ} \mathrm{C}$ for the white light studies). Considering the differences in experimental conditions for the two ARA assays, they are not directly comparable but do contain valuable information about the functional mutants' nitrogenase responsiveness to light.

We have shown that maize are capable of light piping to the roots and that functional mutants of $A$. brasilense having nitrogenase activity show sensitivity to light, most notably red light, with nitrogenase activity being greater than observed in white and blue light and all light conditions showing elevated nitrogenase activity relative to darkness. Since iron plays a large role in the function of the nitrogenase enzyme, we wanted to understand if the presence if light altered the bacteria assimilation of iron micronutrient as ${ }^{59} \mathrm{Fe}^{3+}$ and ${ }^{59} \mathrm{Fe}^{2+}$.

${ }^{59} \mathrm{Fe}^{3+/ 2+}$ Uptake in Functional Mutants of A. brasilense - After subsequent DI water rinses to remove growth media nutrients and resuspension of concentrated bacteria pellets in water, ${ }^{59} \mathrm{Fe}^{3+}$ (ferric) or ${ }^{59} \mathrm{Fe}^{2+}$ (ferrous) was passively distributed to bacteria cultures to assess patterns of 
bacteria iron uptake as a function of nitrogenase activity and auxin biosynthesis, as well as light availability (600 $\mu \mathrm{mol} \mathrm{m} \mathrm{m}^{-2} \mathrm{~s}^{-1}$ intensity) to bacteria over time points ranging 30 minutes to 6 hours incubation time. In terms of mutant strain of bacteria, patterns of iron uptake of both oxidation states were different (Figure 6.2.4.). HM053, the hyper BNF and auxin producing mutant strain, saw a greater uptake of the ferric than ferrous radiotracer (in terms of percent of
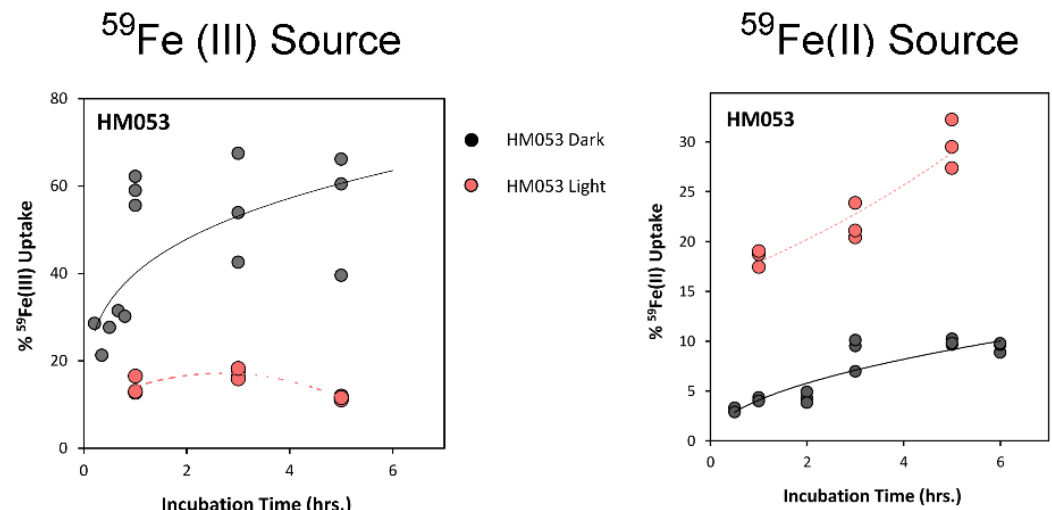
tracer administered) initially
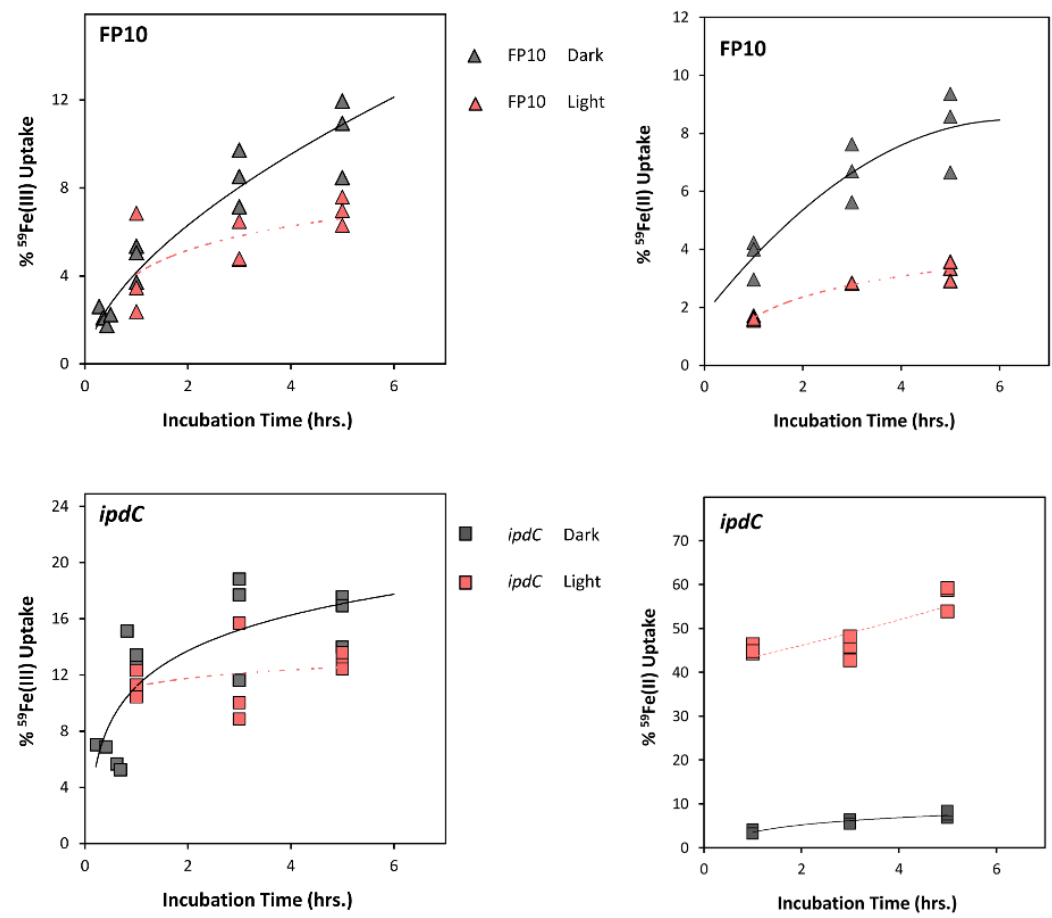

Figure 6.2.4. ${ }^{59} \mathrm{Fe}^{2+/ 3+}$ Radiotracer uptake as a percentage of total tracer administered over time as a function of light status and bacteria biological function. Curves have been inserted to draw the eye.

and over time when grown

and incubated under dark

conditions. In the dark, the

percentage of ${ }^{59} \mathrm{Fe}$ radiotracer

for both oxidation states

taken up by the bacteria

steadily increased over the

window of the study period.

In direct agreement

with this pattern, ipdC, the

functional mutant that had a

significantly reduced capacity

for producing auxin, but

fixed $\mathrm{N}_{2}$ similarly to the

wild-type strain, also saw a

greater uptake of the ${ }^{59} \mathrm{Fe}^{3+}$

than ${ }^{59} \mathrm{Fe}^{2+}$ tracer as a 
percent of administered radiotracer. In terms of overall percentage of ${ }^{59} \mathrm{Fe}^{3+}$ radiotracer taken up (approximately $28-60 \%$ for HM053 and $4-18 \%$ for $i p d C$ ) the two mutant strains were vastly different, while the overall ferrous percentage uptake between the two was relatively similar (410\%). The pattern of radiotracer uptake under dark conditions for FP10 the strain that is deficient in $\mathrm{N}_{2}$-fixation did not follow the trend seen in the other two strains of bacteria. Instead, this strain exhibited an increase over time in percentage of administered uptake of radiotracer but exhibited no difference in the uptake of the ferric vs ferrous radiotracers with overall percentages for both falling within the range observed in the ferrous percent uptake for the HM053 and ipdC functional mutants. It appears that in dark growth conditions, HM053 by far has the greatest ability to assimilate ${ }^{59} \mathrm{Fe}^{3+}$, followed by ipdC then FP10 while no major difference in uptake of ${ }^{59} \mathrm{Fe}^{2+}$ was observed between the mutant strains. This indicates that, in the dark, BNF capacity is correlated to ${ }^{59} \mathrm{Fe}^{3+}$ assimilation by these functional mutants of $A$. brasilense in which that with the greatest BNF capacity takes up the most ${ }^{59} \mathrm{Fe}^{3+}$, while that with the lowest capacity for BNF takes up the least.

Upon growing and incubating the radiotracer with these mutant strains of bacteria in the presence of light, differences in assimilation were noted relative to when they were grown under dark conditions (Figure 6.2.4.). HM053 assimilated less ${ }^{59} \mathrm{Fe}^{3+}$ over time, which is the only instance in which a negative correlation with uptake percentage and time was observed in all the experiments. Additionally, under light conditions HM053 took up more ${ }^{59} \mathrm{Fe}^{2+}$ than ${ }^{59} \mathrm{Fe}^{3+}$ as a percentage of tracer administered (18-32\% for ferrous and $18-12 \%$ for ferric) over 6 hours of incubation. Overall, HM053 took up more ferrous and less ferric radiotracer in the light compared to dark conditions. 
The trends seen in ipdC were slightly different. Ferric radiotracer uptake was similar in the light as it was in the dark, although the slope of the line correlating uptake over time was less steep during light exposure, indicating a slower uptake. What is striking is the trend in ferrous uptake in $i p d C$. In the presence of light stimulus, the ipdC mutant takes up significantly more ferrous radiotracer as a percentage of tracer administered than when grown in dark conditions. This is the same trend as observed with HM053 but much more pronounced.

The FP10 mutant strain follows the same trend as the other bacteria in terms of ferric radiotracer uptake, but has the opposite results in terms of ferrous uptake. The FP10 mutant takes up less ferrous tracer when exposed to light, rather than more as seen in the other mutants, compared to when grown in dark conditions. This is the only functional mutant that lacks the

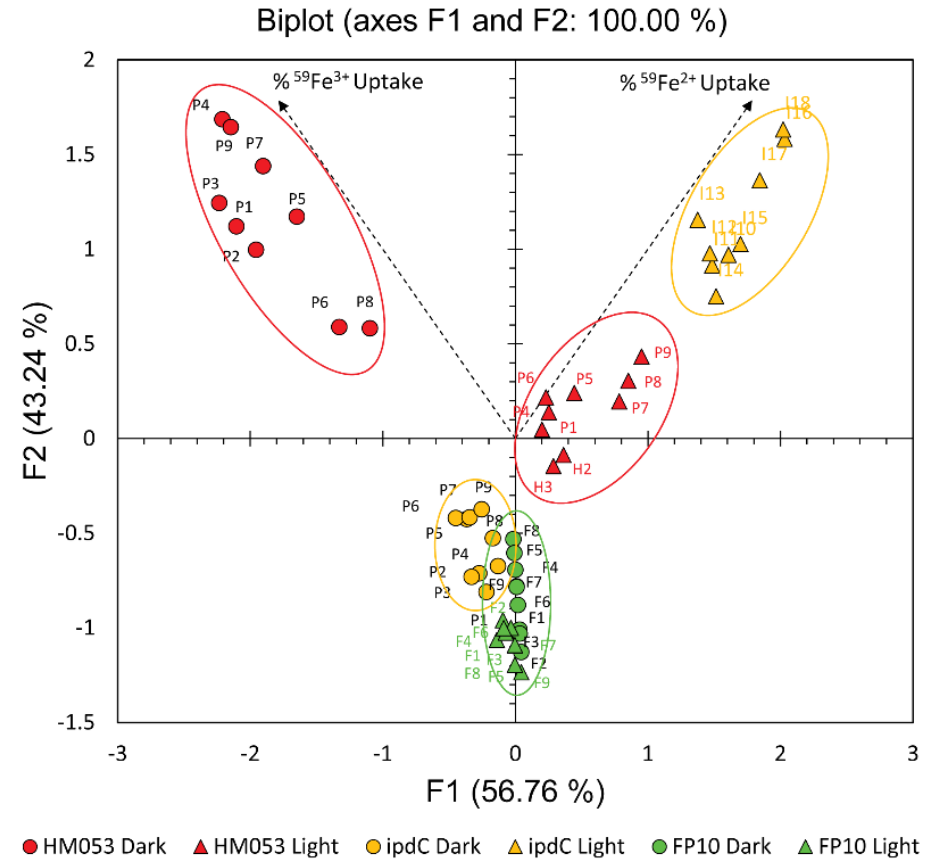

Figure 6.2.5. Principal component analysis of ${ }^{59} \mathrm{Fe}^{2+/ 3+}$ radiotracer uptake data over time as a function of light status and bacteria biological function. Ellipses have been added for visual grouping and do not represent significance in data. capacity for BNF.

Principal Component Analysis was completed on the iron radiotracer uptake data across the treatment types to elucidate trends (Figure 6.2.5.). Here, the information included in our uptake measurements were represented by feature vectors $(\mathrm{F} 1$ and F2) representing $56.76 \%$ and $43.24 \%$ of the information embedded in the data respectively.

Data points from each of the treatment conditions clustered together, indicating behavior within a treatment-type that is 
similar and somewhat distinct from other treatment types. Of greatest note, HM053 mutants in the dark show elevated uptake of ferric and ferrous radiotracer, indicated by the clustering apart from all other treatments and location toward the end of the ferric feature vector and overall positive Y-value. The auxin-deficient mutant, ipdC, shows greatest ferrous radiotracer uptake but this is not observed until exposed to light; otherwise the ipdC mutant clusters near to the FP10 indicating not much difference, other than a slightly elevated ferric uptake, in iron uptake capacity in the dark relative to FP10 in either light condition. The mutant FP10 in both light and dark are nearly indistinguishable from one another and thus do not change under light stimulus. This demonstrates nicely that the ${ }^{59} \mathrm{Fe}$ uptake capacity of the bacteria correlates with BNF capacity of the mutant strain while also showing a light response when BNF capacity is available to the bacteria.

As a major cofactor of the nitrogenase enzyme, differences in Fe needs exhibited by bacteria, which have active and inactive nitrogenase enzymes, makes sense. The oxidation state of iron in the dinitrogenase reductase is primarily ferrous iron with some delocalized $\mathrm{Fe}^{2.5+}$, meaning some ferric iron is present as well126, and HM053 and ipdC both take up relatively large percentages of ferrous radiotracers compared to FP10. In the dark, HM053 takes up mostly ferric iron, as does $i p d C$, which may signal that iron is taken up predominantly in the ferric oxidation state regardless of its ultimate role in metabolism. What is interesting is that predominant uptake changes to ferrous rather than ferric for these two mutant strains under the exposure of light. This leads one to believe that perhaps the BNF capacity of the bacteria plays a role or somehow influences iron uptake of the bacteria and that the uptake of iron in these bacteria can be impacted by the presence or lack of light. Such pronounced metabolic differences in 
micronutrient assimilation and BNF activity lead to question whether other metabolic and physiological processes are being affected by light exposure.

Carbon Metabolism Studies using $\left[{ }^{11} \mathrm{C}_{\mathrm{CO}} \mathrm{O}_{2}\right.$ in mutant strains of A. brasilense reveal differences in carbon resource partitioning correlating to bacteria biological function - A deeper look into the metabolic responses to light in these $A$. brasilense functional mutants was completed using $\left[{ }^{11} \mathrm{C}\right] \mathrm{CO}_{2}$ administered by bubbling through the bacteria liquid culture either in total darkness or under light exposure. First, it was important to understand whether these bacteria can assimilate the radiotracer, and thus measurements were taken based on percent of activity in the centrifuged bacteria pellet from total activity administered decay corrected back to time of administration. As noted in Figure 6.2.6., all of the bacteria were able to assimilate the $\left[{ }^{11} \mathrm{C}\right] \mathrm{CO}_{2}$ radiotracer to a slight, but measurable, degree. HM053 had more variable assimilation values across the spectrum of morning and afternoon as well as lights on and lights off treatments (Figure 6.2.6.). The ipdC mutant saw significantly less assimilation in dark treatments vs light treatments (Figure 6.2.6.), thus is more metabolically active (in terms of carbon usage sourced from $\mathrm{CO}_{2}$ ) when exposed to light. This mimics the enhanced iron uptake as well as enhanced activity of this mutant's nitrogenase enzyme observed under light exposure for this functional mutant. Lastly, the FP10 mutant showed, again, different behavior relative to the BNF-capable mutants. FP10 assimilated the least amount of $\left[{ }^{11} \mathrm{C}\right] \mathrm{CO}_{2}$ across all treatment types and saw a slight and significant increase in radiotracer assimilation under dark conditions in the afternoon (Figure 6.2.6.). Since the $\mathrm{HM} 053$ mutant had such variable $\left[{ }^{11} \mathrm{C}\right] \mathrm{CO}_{2}$ assimilation values, no clear trend can be drawn between the nitrogen fixation capacity, light exposure, and carbon metabolism of $\mathrm{CO}_{2}$. What can be concluded is that those with nitrogenase activity assimilate more $\left[{ }^{11} \mathrm{C}\right] \mathrm{CO}_{2}$ 
than those without, and that the $i p d C$ mutant is more active in assimilation under light conditions than dark.

The alteration in each functional mutant's carbon dioxide assimilation percentage at different times of day hints at the presence of a circadian rhythm or effect taking place within the bacteria. Indeed, there is evidence in the literature for circadian rhythms observed in Rhizobacteria communities wherein the microbial community composition was altered with plant exposure (in this case, Arabidopsis thaliana) to diurnal cycling ${ }^{34}$. While this was not investigated in respect to Azospirillum brasilense specifically, we report in these $\left[{ }^{11} \mathrm{C}\right] \mathrm{CO}_{2}$ studies that these functional mutants exhibit consistent differences in assimilation of $\mathrm{CO}_{2}$ and subsequent metabolic patterns not only when exposed to light or total darkness, but also as a function of time of day (morning being $9 \mathrm{AM}\left[{ }^{11} \mathrm{C}\right] \mathrm{CO}_{2}$ administration, and $\mathrm{PM}$ being $1 \mathrm{PM}$ administration).
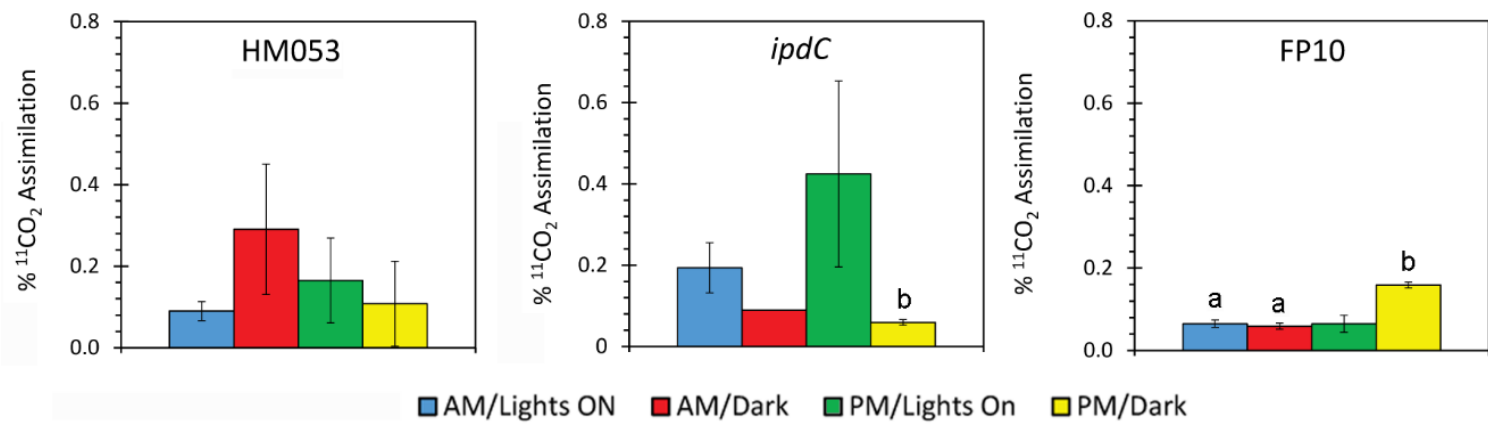

Figure 6.2.6.- Percent Assimilation of ${ }^{11} \mathrm{CO}_{2}$ in bacteria liquid cultures in darkness and exposed to white lights across morning and afternoon. Statistical significance is shown on the figure $(\mathrm{p}<0.05)$ and is designated with an ' $a$ ' when compared across functional mutants between FP10 and HM053 and with a ' $b$ ' when compared within the same mutant but between light exposure and time of day.

Knowing the bacteria were assimilating the $\left[{ }^{11} \mathrm{C}\right] \mathrm{CO}_{2}$ radiotracer, it became desirable to examine changes in microbial ${ }^{11} \mathrm{C}$ metabolism from this source to determine it changes with time of day and light conditions. As mentioned in the Methods section of this Chapter, the metabolic landscape depicting whole pools of different metabolite classes could be teased apart using solid- 
phase extractions coupled with gamma counting. For each functional mutant of bacteria, a metabolism pie chart was compiled for comparison across time of day and light exposure. HM053 showed a significant increase in acidic metabolite formation when grown and incubated with the radiotracer in dark conditions relative to exposure to white light LED (Figure 6.2.7.) in

the morning. This was

accompanied with a significant

decrease in hydrophobic

metabolites measured. When a

comparison was made with white

light exposed treatments between

morning and afternoon

incubations with the radiotracer,

this same trend was observed with

an additional significant increase

is basic metabolite production.

Bacteria cultures of HM053 grown

and incubated with the radiotracer

in the dark showed a significant

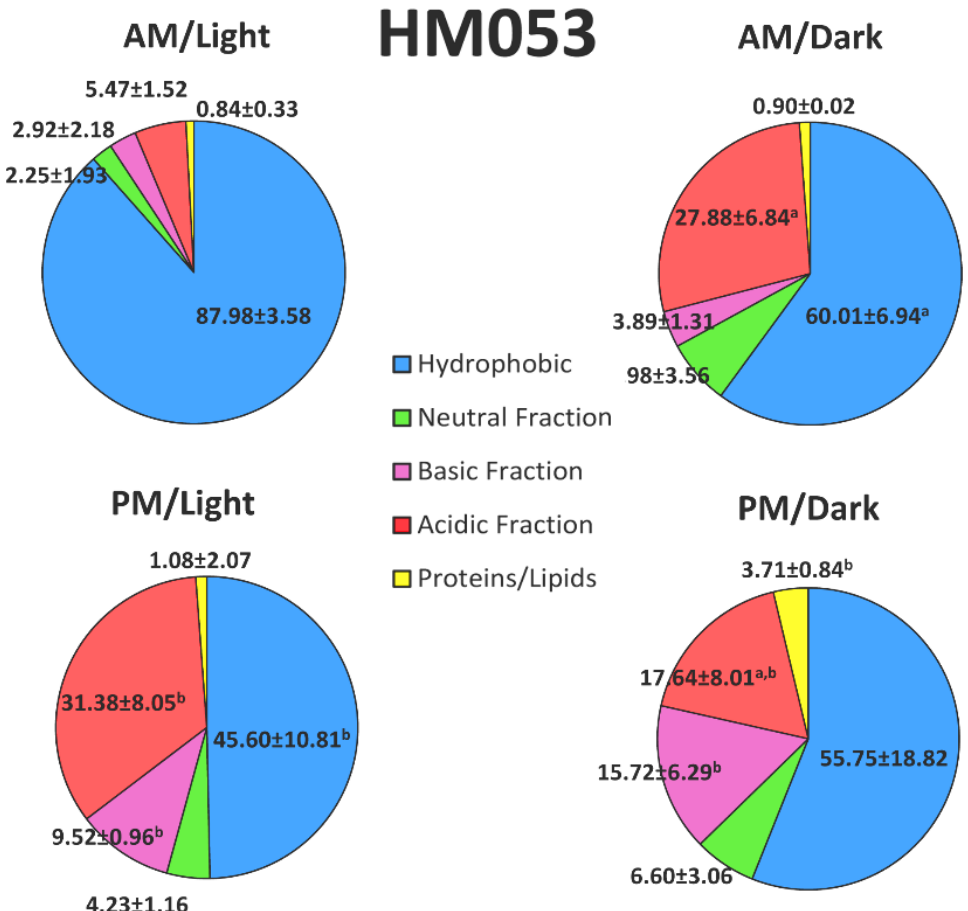

Figure 6.2.7.- ${ }^{11} \mathrm{CO}_{2}$ Metabolism Charts for the $\mathrm{HM053}$ functional mutant of $\boldsymbol{A}$. brasilense Statistical significance is shown on the figure ( $p>0.05)$ and is designated with an ' $a$ ' when compared across functional mutants and with a ' $b$ ' when compared within the same mutant but between light exposure and time of day.

increase in production of radio-labeled proteins and lipids as well as basic components along with a significant decrease in acidic components. It is clear from examining the metabolic landscape that HM053 prioritized acidic metabolites most when exposed to white light, and in the dark that mutant generated more basic and neutral metabolites in addition to the acidic components (Figure 6.2.7.). Changes in light exposure had clear and significant impacts on the 
bacteria's carbon metabolism. This observation combined with the ARA and iron uptake data suggests these bacteria are sensitive to light and adjust their physiological and metabolic processes based on its absence and presence.

The ipdC mutant showed fewer pronounced differences in carbon metabolism between white light and dark growth and radiotracer incubation conditions (Figure 6.2.8.) relative to HM053. The only significant difference observed in this mutant is the enhancement of the basic fraction of metabolites observed in the dark relative to white light exposure in the morning. FP10 also saw minimal changes in radiolabeled metabolism between light and dark exposure across morning and night radiotracer incubation (Figure 6.2.9.). There are significant decreases

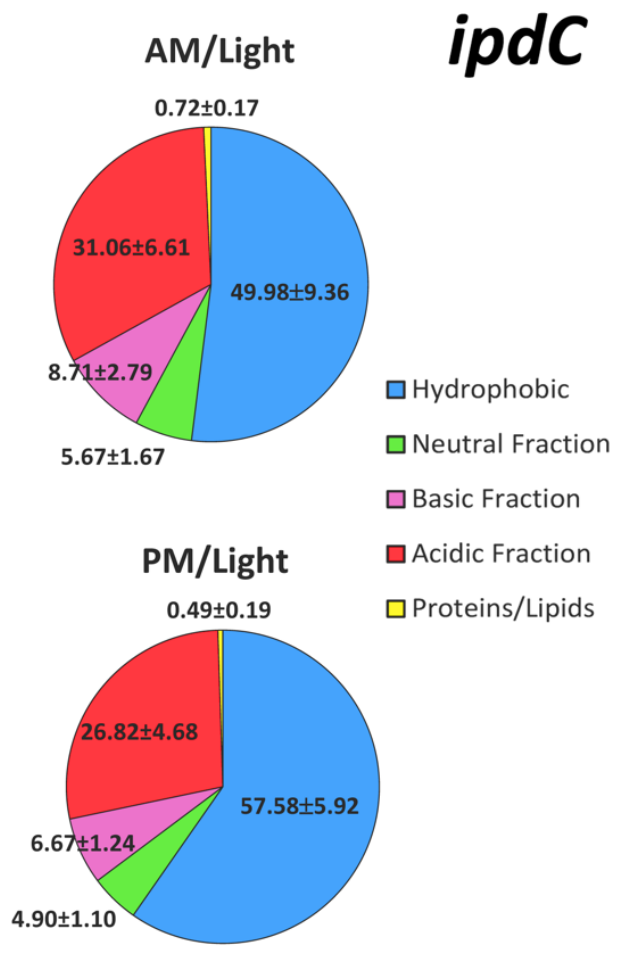

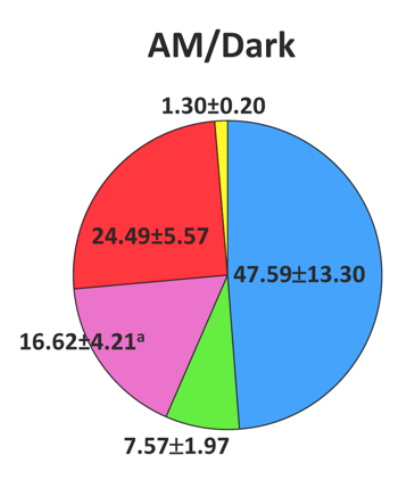

PM/Dark

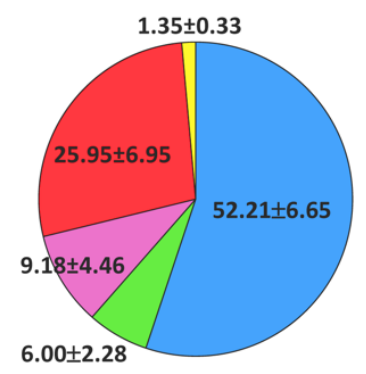
in production of proteins and lipids as well as basic metabolites when FP10 is treated in darkness relative to white light in the morning. There are no differences in carbon dioxide metabolism between morning and afternoon tracer administration for the white light exposed bacteria cultures, but in the dark, FP10

Figure 6.2.8.- ${ }^{11} \mathrm{CO}_{2}$ Metabolism Charts for the ipdC functional mutant of $\boldsymbol{A}$. brasilense. Statistical significance is shown on the figure ( $\mathrm{p}>0.05)$ and is designated with an ' $a$ ' when compared across functional mutants and with a ' $b$ ' when compared within the same mutant but between light exposure and time of day.

greatly enhances its metabolism of basic metabolites when incubated in the radiotracer in 
the afternoon relative to morning

incubation. While there were

differences in metabolism noted

under light and dark treatments

for the auxin deficient (ipdC)

and BNF-deficient mutant

(FP10) bacteria strains, it was

not as strong as the differences

observed in the hyper-BNF and

auxin producing strain, HM053.

Still, significant differences

were observed in all functional

mutants to some degree and
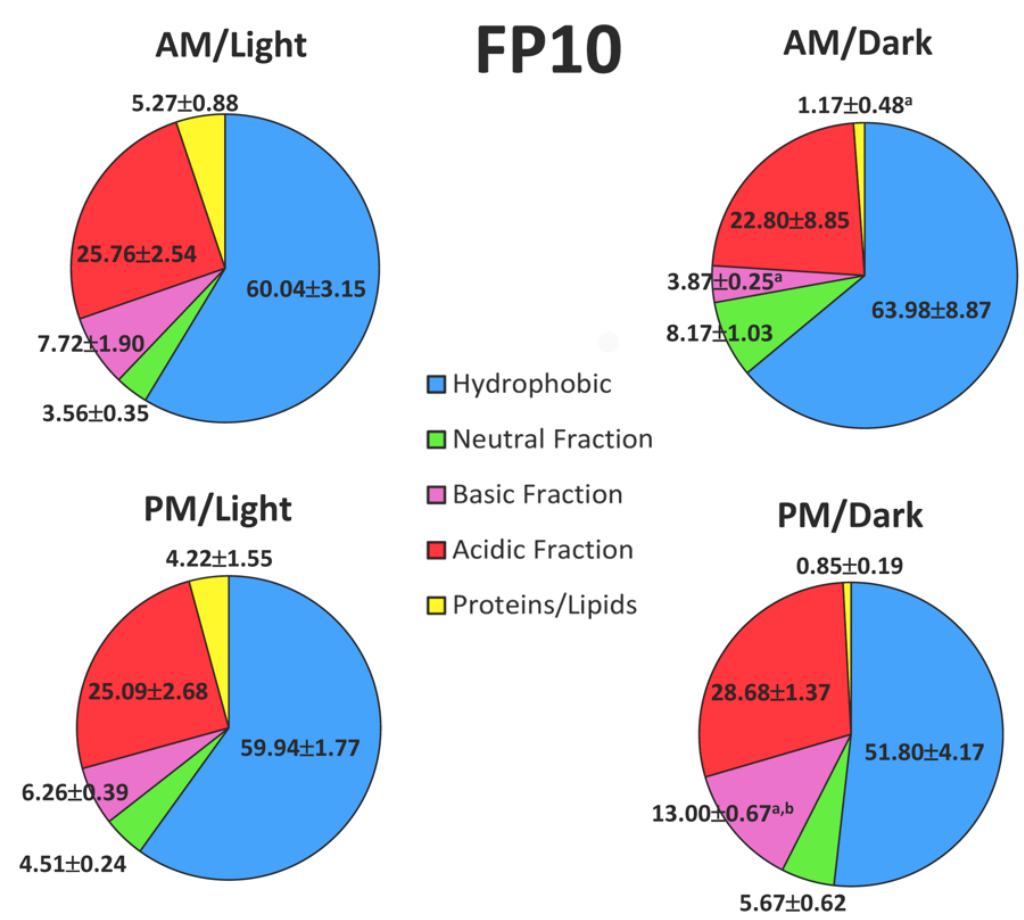

Figure 6.2.9.- ${ }^{11} \mathrm{CO}_{2}$ Metabolism Charts for the FP10

functional mutant of $\boldsymbol{A}$. brasilense. Statistical significance is shown on the figure $(\mathrm{p}>0.05)$ and is designated with an ' $\mathrm{a}$ ' when compared across functional mutants and with a ' $b$ ' when compared within the same mutant but between light exposure and time of day.

indicate once again that they are sensitive, or responsive, to light stimulus.

\subsection{Conclusion}

As the increased implementation of bacterial inoculants for the enhancement of agricultural crop yield and plant nutrition continues, it becomes more important to fully understand the biological functions and mechanisms of action behind their usefulness. Here, we have investigated the iron uptake of these bacteria functional mutants as ferric and ferrous iron over time and as a function of light exposure. Iron uptake correlated strongly with BNF capacity of the functional mutants, with HM053 taking up the most iron (especially of ferric) and FP10 taking up the least (including both ferric and ferrous). Additionally, the mutants with BNF capacity showed a stronger light response, taking up more iron overall when exposed to white 
light relative to darkness. Iron is directly involved in the BNF capacity as a cofactor of the nitrogenase enzyme. Indeed, when assessing the activity of the nitrogenase enzymes of the bacteria mutant strains when exposed to light vs dark with ARA, the HM053 showed greatest nitrogenase activity under exposure to white light relative to darkness, with ipdC showing the same overall trend but with lower levels of nitrogenase activity. FP10 did not show activity nor a light vs dark change in response. When a similar experiment was run for HM053 and ipdC in red and blue light conditions, both of these mutants showed a much higher response to the red light than blue. This indicates the functional mutants, especially those with BNF activity, are influenced by light, especially red light. Such knowledge can become relevant across many crop enhancement applications; for example, scientists developing $\mathrm{N}_{2}$ fixation capabilities in grain crops could consider uncovering more about the process of light piping and look to promote light piping for elevated BNF activity.

Our work with the $\left[{ }^{11} \mathrm{C}\right] \mathrm{CO}_{2}$ radiotracer shows that these functional mutants of Azospirillum brasilense do assimilate carbon dioxide and metabolize it into various biologically relevant metabolite classes. Though only identified by class of metabolite based on overarching chemical properties, it is also clear that metabolism of carbon dioxide in these mutants can be influenced by presence of light, which indicates a level of light response shared across the bacteria functional mutants. While this sensitivity to light can be noted to some degree in all mutants, it is most pronounced in the HM053 mutant which has the greatest capacity for BNF and auxin production. Our group has begun the process of radiosynthesizing $\left[{ }^{11} \mathrm{C}\right]$-lactic acid, a more common carbon source for $A$. brasilense bacteria, and in future work it would be illuminating to repeat these metabolite profiles using that radiotracer to investigate how differently these strains utilize that carbon source under the differing light growth conditions. It 
is possible new trends could emerge that aid in an explanation of the enhancement of iron uptake in HM053 and ipdC in white light while no enhancement is noted for FP10.

\subsection{Materials and Methods}

Light piping investigations with Zea mays- In order to investigate whether light conduction occurs in maize a young maize plant was set up in a black box as previously described (Figure 6.2.3.). The upper stem was cut and inserted it into an aluminum lined black light tight plastic tube which helped conduct light from a fiber optic halogen light source through an infrared filter, cutting out wavelengths below $720 \mathrm{~nm}$. Plumbers putty attached the stem to the dark box, making for a light tight seal. One of the crown roots was chosen for examination and the end of the root was cleanly cut with a razor blade. That cut root end was mounted facing a digital single lens reflex (DSLR) EOS 3D Mark III Canon camera brought to focus with the camera's front macro lens (MP-E $65 \mathrm{~mm} \mathrm{f/2.8} \mathrm{1-5x)} \mathrm{by} \mathrm{allowing} \mathrm{ambient} \mathrm{light} \mathrm{to} \mathrm{leak} \mathrm{into} \mathrm{the} \mathrm{dark} \mathrm{box} \mathrm{to}$ produce the brightfield root end image (Figure 6.2.1.). Then the box was sealed with light tight tape and the halogen light was turned on. The exposure setting was 300 seconds with an iso setting of 100. IR light conducted through the stem and roots was picked up by the optical system, which included an AstroScope night vision image intensifier tube for DSLR camera (Figure 6.4.1.).

Plant growth for light piping studies- Maize kernels from Elk Mound Seed Co. (Hybrid 8100) were dark germinated at room temperature for two days on sterilized paper towels wetted with sterile water in a petri-dish. Seeds were transplanted to a growth pouch wetted with sterile Hoagland's basal salt solution for approximately one week. They were then transferred to eightinch plastic cones filled with Turface ${ }^{\mathrm{TM}}$ (expanded clay matrix) where the bottom portion of the cone was immersed in de-ionized water. Nutrient was introduced as Hoagland's solution every 
three days. Growth conditions consisted of 12-hour photoperiods, $500 \mu \mathrm{mol} \mathrm{m}^{-2} \mathrm{~s}^{-1}$ light intensity, and temperatures of $25^{\circ} \mathrm{C} / 20^{\circ} \mathrm{C}$ (light/dark) with humidity at $70-80 \%$ for two weeks.

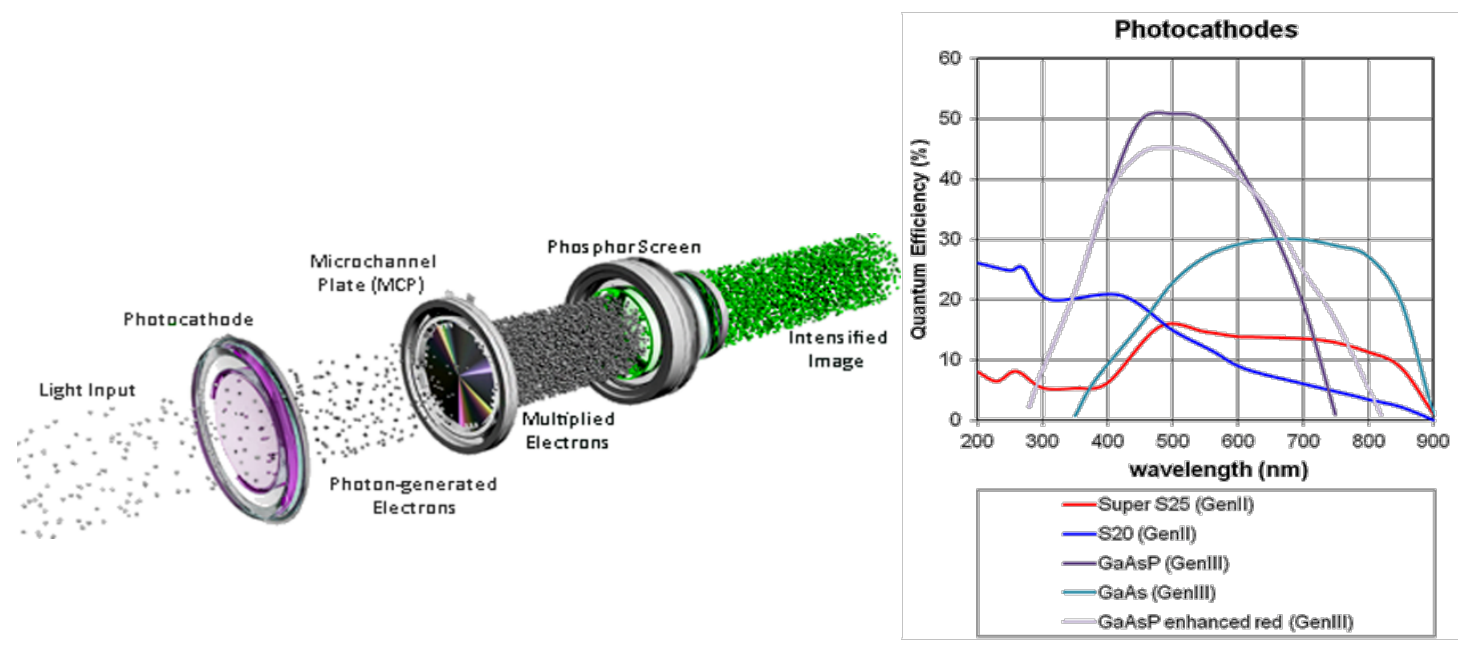

Figure 6.4.1. Image Intensifier for capturing light piping in maize. Our image intensifier was Sofradir EC9350-CCD-PRO Image Intensifier marketed by B\&H Photo under the Astroscope brand. This tube was configured to operate on Canon DSLR camera and inserted between the Canon lens and the camera. The photocathode was the GaAs (Gen III) type sensitive to light mapping the turquoise curve. Hence it was sensitive to the IR light that the plant conducted. At the core of the intensifier tube is a photocathode that converts photons to electrons which can be multiplied while maintaining the 2D spatial position with the microchannel plate. These electrons strike a phosphor screen giving off green light at a huge boost in output signal that the camera sensor can record.

Bacteria Growth- Three functional mutant strains of Azospirillum brasilense (HM053, ipdC, and FP10) were used in the study and obtained through a material transfer agreement between the Federal University of Paraña (UFPR, Curitiba, PR 174CEP 81531-980, Brazil) and the corresponding author's institution. The HM053 mutant is a natural mutant of the wild-type strain of $A$. brasilense FP2 (Sp7 ATCC $29145 \mathrm{Nif}^{+} \mathrm{Sm}^{\mathrm{r}} \mathrm{Nal}^{\mathrm{r}}$ ) screened through its resistance to ethylenediamine $\left(\mathrm{EDA}^{\mathrm{r}}\right)^{35} 36$. For the ipdC mutant, the indole-3-pyruvate decarboxylase gene (ipdC), coding for a key enzyme of the indole-3-pyruvic acid pathway of auxin (indole-3acetic acid) biosynthesis in Azospirillum brasilense, was functionally disrupted in a site-specific manner using a SacB-cassette insertion into the ipdC gene of wild-type FP2 (Sp7 ATCC 29145) followed by homologous recombination. The method allowed for the construction of 
the ipdC mutation strain without unwanted sequence changes and relied on the $\lambda$ Red recombineering method (Direct and Inverted Repeat stimulated excision; DIRex) which works well for generating single point mutations, small insertions or replacements as well as deletions of any size, in a bacterial gene ${ }^{37}$. The resultant knock-out strain exhibited significant reduction in auxin biosynthesis to a level of $10 \%$ that of the wild-type strain (Personal communication). The FP10 mutant was obtained by N-nitrosoguanidine mutagenesis of the FP2 wild-type strain of A. brasilense and isolated by growth on glutamate medium ${ }^{38}$.

The functional mutants were grown in liquid NFbHP-lactate medium following published procedures ${ }^{22}$. The medium contained $20 \mathrm{mM}$ ammonium chloride $\left(\mathrm{NH}_{4} \mathrm{Cl}\right)$ as a nitrogen source and streptomycin antibiotic $\left(80 \mu \mathrm{gL}^{-1}\right)$. The cultures were grown in a shaking incubator at $30^{\circ} \mathrm{C}$ and $130 \mathrm{rpm}$ in $10 \mathrm{~mL}$ volume, then re-propagated into $40 \mathrm{~mL}$ volume approximately 24 hrs prior to starting the tracer administration. The bacteria were grown under two treatment types: 24-hr light and 24-hr dark. During the light experiments, bacteria were propagated directly under a 12'x12' red-blue LED light panel intended for indoor plant growth $\left(141.50 \mu \mathrm{mol} \mathrm{m}{ }^{-2} \mathrm{~s}^{-1}\right.$ light intensity) that was positioned over the shaking incubator. Lights were left on for the entirety of the bacteria growth period. Dark experiments involved propagation of the bacteria in the dark, handled under indirect light, and grown in the shaking incubator wrapped completely in aluminum foil to shield from any ambient light. Acetylene Reduction Assay Studies- Acetylene reduction assays were completed to assess functionality of the nitrogenase enzyme under light and dark growing conditions and exposure. Calcium carbide $(1 \mathrm{~g})$ was placed in a clean and dry $50 \mathrm{~mL}$ volume Falcon Tube. The cap to the Falcon Tube was modified to house a Burrell seal port for connecting a $60 \mathrm{~mL}$ volume syringe filled with $30 \mathrm{~mL}$ DI water and backfilled with $30 \mathrm{~mL}$ air for pushing the acetylene out of the 
Falcon Tube. The cap also held another port for connecting soft silicon rubber tubing connected to the collection bag (Figure 6.4.2). The plastic collection pouch was equipped with a plastic stem that, when pushed in, acted like a valve and closed. The pouch also had a silicon rubber septum built in for syringe sampling of the gas.

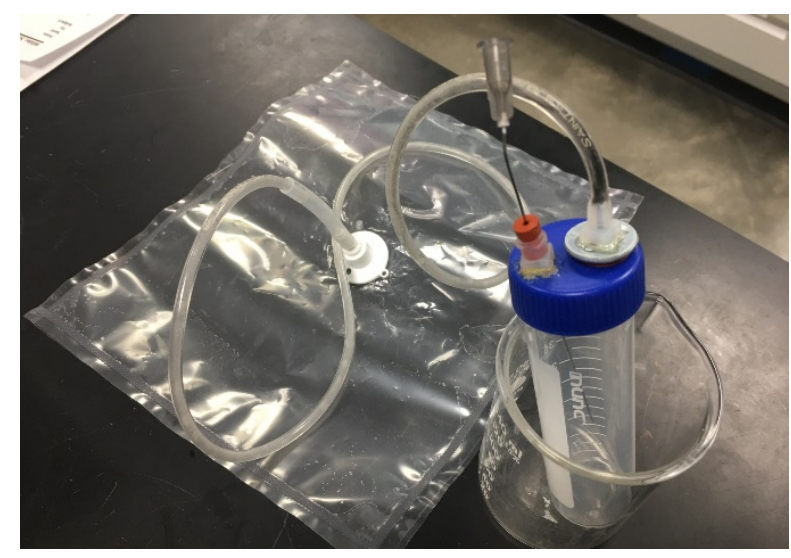

Figure 6.4.2- Experimental set up for Acetylene Reduction Assay.

There was a tendency to puncture the plastic bag, thus acquisition of syringe samples of acetylene gas occurred using another small section of silicon rubber tubing with a Burrell seal connected to the end.

The bacteria were grown in association with maize roots, which were harvested, weighed (typically $1 \mathrm{gfw}$ ) and placed in a 500$\mathrm{mL}$ mason jar where the top was modified with a gas sampling port and an acetylene addition port (Figure 6.4.3.). The latter had inside tubing (1/16" Teflon) that extended to the bottom of the jar. Once the roots were sealed inside, $30 \mathrm{~mL}$ of acetylene was withdrawn from the plastic pouch using a gas tight syringe and injected into the Mason jar through the inlet port. A syringe needle was temporarily placed in the other port to vent the jar of excess gas while loading with acetylene and later removed during incubation. Samples $(0.1 \mathrm{~mL})$ were removed at different incubation times for analysis of ethylene production using gas chromatography and were injected

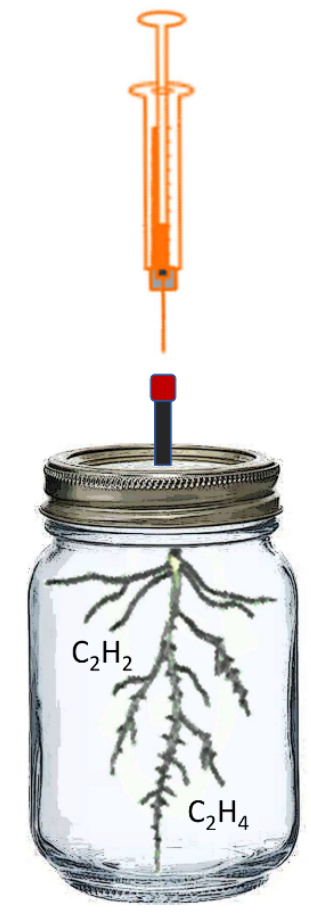

Figure 6.4.3.Experimental set up for Acetylene Reduction Assay root incubation chamber and sampling port. into a Hewlett Packard 5890A gas chromatograph equipped with a 2-m long, 1-mm inner 
diameter ShinCarbon ST packed column and flame ionization detector. Column temperature was programmed as follows: the program started with a two-minute hold at $40{ }^{\circ} \mathrm{C}$ and then increased $10{ }^{\circ} \mathrm{C} \mathrm{min}-1$ to $250{ }^{\circ} \mathrm{C}$. The injector temperature was maintained at $250{ }^{\circ} \mathrm{C}$ and the flame ionization detector temperature was maintained at $300^{\circ} \mathrm{C}$. Chromatographic peaks for ethylene were measured using PeakSimple ${ }^{\mathrm{TM}}$ chromatography software and quantified against ethylene standards. Ethylene elutes after acetylene at a retention time of around 13 minutes. Bacteria ${ }^{59} \mathrm{Fe}$ Assimilation Studies- Cultures were incubated for Fe-59 studies 24 hours prior to start of study. On the day of the experiment, the cultures were centrifuged, washed with water, and combined to yield a concentrated pellet of bacteria that was resuspended in $11 \mathrm{~mL}$ of DI water. A UV reading was taken at $600 \mathrm{~nm}$ using $2 \mathrm{~mL}$ of the $11 \mathrm{~mL}$ sample. Of the $9 \mathrm{~mL}$ bacteria sample remaining, 9 aliquots of $1 \mathrm{~mL}$ were placed into $15-\mathrm{mL}$ labeled falcon tubes. The ${ }^{59} \mathrm{Fe}^{2+/ 3+}$ radiotracer was added to each falcon tube $\left(3.6 \mathrm{~mL}, 20 \mu \mathrm{Ci}\right.$ or $\left.7.4 \times 10^{5} \mathrm{~Bq}\right)$ and start times were recorded. A $36.0 \mu \mathrm{L}$ sample of the ${ }^{59} \mathrm{Fe}$ radiotracer used was counted in the $\mathrm{NaI}(\mathrm{Tl})$ gamma well counter and corrected for volume and detector efficiency back to a value for activity administered to each falcon tube. Samples were incubated on a rocking platform for 1,3 , and 5 hr time points either under LED lights or wrapped in aluminum foil for dark studies.

At each time point, the appropriate samples were centrifuged, washed with water three times, and re-suspended in $1 \mathrm{~mL}$ of $1 \mathrm{M} \mathrm{HCl}$. An aliquot $(100 \mu \mathrm{L})$ of each were removed for activity measurements in the $\mathrm{NaI}(\mathrm{Tl})$ gamma well counter for a measure of activity before extraction. The remainder of the washed bacteria were cell disrupted for 2 minutes under $100 \%$ amplitude to burst cells for Fe-59 extraction. Once complete, samples were centrifuged 2 minutes and the supernatant was removed; $100 \mu \mathrm{L}$ of the supernatant was removed for immediate counting in the $\mathrm{NaI}(\mathrm{Tl})$ gamma well detector while the remaining $900 \mu \mathrm{L}$ was used 
for iron oxidation state speciation via ion chromatography (describe system). The ${ }^{59} \mathrm{Fe}^{3+}$ and ${ }^{59} \mathrm{Fe}^{2+}$ peaks were caught after separation on the analytical column and distribution of activity in each oxidation was measured with the $\mathrm{NaI}(\mathrm{Tl})$ gamma well detector. The percent tracer uptake was calculated for each bacterial functional mutant under light and dark conditions at 1,3 and 5hour intervals. Additionally, the ratio between ${ }^{59} \mathrm{Fe}^{3+}$ and ${ }^{59} \mathrm{Fe}^{2+}$ was calculated to determine if conversion of the oxidation state of the tracer administered remained the same or was altered by the system or by the bacteria functional mutants. Bacteria $\left[{ }^{11} \mathrm{C}\right] \mathrm{CO}_{2}$ Assimilation and Metabolism Studies- $\left[{ }^{11} \mathrm{C}\right] \mathrm{CO}_{2}\left({ }^{11} \mathrm{C} \mathrm{t} / 2=20.4 \mathrm{~min}\right)$ was produced on the GE PETrace Cyclotron at the Missouri Research Reactor Center using a highpressure research grade ${ }^{14} \mathrm{~N}_{2}$ gas target irradiated using a $16.4 \mathrm{MeV}$ proton beam to generate ${ }^{11} \mathrm{C}$ via the ${ }^{14} \mathrm{~N}(\mathrm{p}, \alpha){ }^{11} \mathrm{C}$ nuclear reaction. The generated $\left[{ }^{11} \mathrm{C}\right] \mathrm{CO}_{2}\left(\sim 100 \mathrm{mCi}\right.$ or $\left.3.7 \times 10^{9} \mathrm{~Bq}\right)$ was trapped on a $4 \AA$ molecular sieve at room temperature then desorbed above approximately $250{ }^{\circ} \mathrm{C}$ into an air stream at $200 \mathrm{~mL} \mathrm{~min}^{-1}$ as a discrete pulse for labeling. Each bacterial sample was pulse-fed $\left[{ }^{11} \mathrm{C}\right] \mathrm{CO}_{2}$ until a maximum activity was detected, then the flow was stopped for 10 minutes to allow incubation of the bacteria with tracer. Following the incubation, the sample was chased with auxiliary air for 10 minutes before the air flow was stopped again for sample extraction.

The bacteria were harvested by pouring the culture into a $50 \mathrm{~mL}$ falcon tube and centrifuging for 5 minutes at $4.4 \mathrm{rpm}$ until a bacteria pellet formed in the tube. Bacteria grown under dark conditions had their falcon tubes wrapped in aluminum foil. The supernatant was removed and discarded, and sterile water was used to re-suspend and wash the pellet of leftover culture. Each culture of bacteria was washed twice prior to absorbance readings in the Azura UVD 2.15 Knauer UV-VIS spectrometer. At the final wash step, the pellet was suspended in 
only $10 \mathrm{~mL}$ of sterile water and a $2-\mathrm{mL}$ representative was placed in a cuvette for absorption measurement at $600 \mathrm{~nm}$. The measured bacteria were returned to the falcon tube and the bacteria solution was diluted to $40 \mathrm{~mL}$ total volume with sterile water. A cap was placed on the falcon tube, which allowed air flow into the tube through an aquarium bubbler and outflow toward an ascarite trap (Figure 6.4.4.).

The sample tube was placed in a lead lined radiotracer administration stall and was suspended in front of a PIN diode

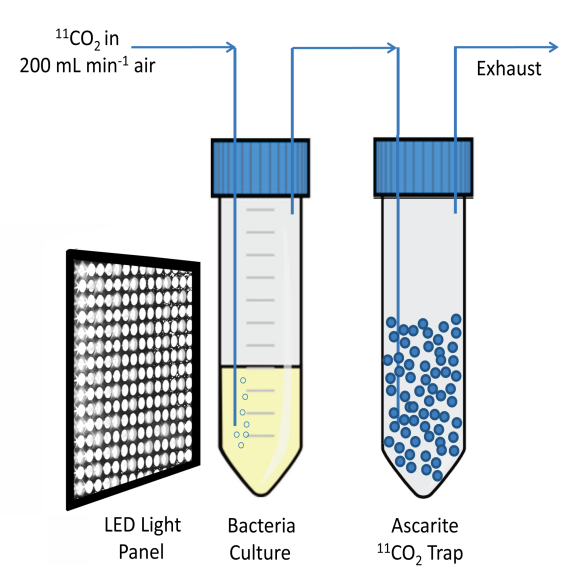

Figure 6.4.4. Experimental set-up for $\left[{ }^{11} \mathrm{C}\right] \mathrm{CO}_{2}$ assimilation studies.

radiation detector (Carroll Ramsey Associates, Berkeley, CA 94710, USA) enabling continuous measurement of radioactivity levels within the cell during the initial pulse and in the minutes after administration. The bacteria sample falcon tube was wrapped in aluminum foil if a dark treatment was being performed, but if a light treatment was being performed, LED lights (550 $\mu \mathrm{mol} \mathrm{m} \mathrm{m}^{-2} \mathrm{~s}^{-1}$ light intensity) lining the radiotracer stall ceiling as well as an additional LED light panel angled directly at the tube were utilized. The ascarite trap connected by tubing to the bacterial sample was placed within a lead pig and buried beneath lead bricks to reduce dose and background measured by the detector attached to the sample.

After $\left[{ }^{11} \mathrm{C}\right] \mathrm{CO}_{2}$ pulsing, incubation, and clearing the bacteria sample was removed from the set up and the bubbler cap was replaced with a solid cap. The sample was moved in a lead pig for personnel safety. The bacteria were first centrifuged for 5 minutes and moved to a radiation hood where the supernatant was removed and stored in a $100 \mathrm{~mL}$ plastic bottle. An 
additional $25 \mathrm{~mL}$ of sterile water was added and the pellet was resuspended before a second centrifugation step. The supernatant was removed as previously describe and stored in the same plastic bottle. Radiation measurements of both the pellet and the supernatant water were taken in the Capintec Dose Calibrator for subsequent C-11 carbon fixation calculations. All data was decay corrected back to the time of the $\left[{ }^{11} \mathrm{C}\right] \mathrm{CO}_{2}$ tracer pulse.

A 60:40 methanol: water solvent $(1 \mathrm{~mL})$ was then added to the bacteria pellet and transferred to an Eppendorf ${ }^{\mathrm{TM}}$ tube using a glass pipet. The content was vortexed (VWR analog vortex mixer; Sigma-Aldrich Corp., St. Louis, MO, USA) then sonicated (Branson Bransonic 32; Sigma-Aldrich Corp. St. Louis, MO, USA) for 2 min. The Eppendorf ${ }^{\mathrm{TM}}$ tube was centrifuged 2 $\min$ at $15,000 \mathrm{rpm}$ to separate insoluble from soluble portions. The supernatant (soluble fraction) was removed to a separate tube. Both the insoluble fraction and a $20 \mu \mathrm{L}$ aliquot of the soluble fraction were measured for ${ }^{11} \mathrm{C}$-activity using a $\mathrm{NaI}(\mathrm{Tl})$ gamma counter to relate back to total fixed $\left[{ }^{11} \mathrm{C}\right] \mathrm{CO}_{2}$.

The supernatant was further separated for Sep-Pak ${ }^{\mathrm{TM}}$ analysis. A $300 \mu \mathrm{L}$ aliquot was processed through a QMA Light Sep-Pak ${ }^{\mathrm{TM}}$ and attached SCX cartridge for quantification of acidic, basic, and neutral components. The QMA and SCX were prepared with a $10 \mathrm{~mL}$ rinse of DI water followed by $10 \mathrm{~mL}$ of air. Following addition of the $300 \mu \mathrm{L}$ supernatant, the cartridges are eluted with $10 \mathrm{~mL}$ of DI water which is collected upon elution. The QMA represents the acidic components as it captures positively charged species, while the SCX captures the negatively charged, or basic, chemical species. A $1 \mathrm{~mL}$ aliquot of the $10 \mathrm{~mL}$ DI water rinse is taken and counted to represent the non-charged, or neutral, chemical species.

A second $300 \mu \mathrm{L}$ aliquot of supernatant was processed through a C-18 Sep-Pak for quantification of lipid and protein components. The C-18 Sep-Pak was prepared with $5 \mathrm{~mL}$ of a 
$0.1 \%$ trifluoroacetic acid (TFA) in DI water solution, followed by $5 \mathrm{~mL}$ of an $80: 200.1 \%$ TFA: methanol solution, and then $10 \mathrm{~mL}$ of air. After the addition of the $300 \mu \mathrm{L}$ aliquot of soluble supernatant, the Sep-Pak was eluted with $1 \mathrm{~mL}$ of a 70: 30 0.1\%TFA: methanol solution into waste. All the cartridges were placed in individual plastic bags and measured in the $\mathrm{NaI}(\mathrm{Tl})$ gamma counter and all radiation measurements were decay corrected back to the time of the $\left[{ }^{11} \mathrm{C}\right] \mathrm{CO}_{2}$ tracer pulse.

\subsection{Abbreviations}

PGPB Plant Growth Promoting Bacteria

GA Giberillic Acid

PHY Photoreceptor

CA Carbonic Anhydrases

BNF Biological Nitrogen Fixation

ARA Acetylene Reduction Assay 


\section{References}

(1) Kumar, S.; Kateriya, S.; Singh, V. S.; Tanwar, M.; Agarwal, S.; Singh, H.; Khurana, J. P.; Amla, D. V.; Tripathi, A. K. Bacteriophytochrome Controls Carotenoid-Independent Response to Photodynamic Stress in a Non-Photosynthetic Rhizobacterium, Azospirillum Brasilense Sp7. Scientific Reports 2012, 2 (1), 872.

(2) Lee, H.-J.; Ha, J.-H.; Kim, S.-G.; Choi, H.-K.; Kim, Z. H.; Han, Y.-J.; Kim, J.-I.; Oh, Y.; Fragoso, V.; Shin, K.; Hyeon, T.; Choi, H.-G.; Oh, K.-H.; Baldwin, I. T.; Park, C.-M. StemPiped Light Activates Phytochrome B to Trigger Light Responses in Arabidopsis Thaliana Roots. Sci. Signal. 2016, 9 (452), ra106-ra106.

(3) Gelderen, K. van; Kang, C.; Pierik, R. Light Signaling, Root Development, and Plasticity. Plant Physiology 2018, 176 (2), 1049-1060.

(4) Kircher, S.; Schopfer, P. Photosynthetic Sucrose Acts as Cotyledon-Derived Long-Distance Signal to Control Root Growth during Early Seedling Development in Arabidopsis. PNAS 2012, 109 (28), 11217-11221.

(5) Regnault, T.; Davière, J.-M.; Wild, M.; Sakvarelidze-Achard, L.; Heintz, D.; Carrera Bergua, E.; Lopez Diaz, I.; Gong, F.; Hedden, P.; Achard, P. The Gibberellin Precursor GA 12 Acts as a Long-Distance Growth Signal in Arabidopsis. Nature Plants 2015, 1 (6), 1-6.

(6) Chen, X.; Yao, Q.; Gao, X.; Jiang, C.; Harberd, N. P.; Fu, X. Shoot-to-Root Mobile Transcription Factor HY5 Coordinates Plant Carbon and Nitrogen Acquisition. Current Biology 2016, 26 (5), 640-646.

(7) Kiss, J. Z.; Correll, M. J.; Mullen, J. L.; Hangarter, R. P.; Edelmann, R. E. Root Phototropism: How Light and Gravity Interact in Shaping Plant Form. Gravit Space Biol Bull 2003, $16(2), 55-60$.

(8) Kakuszi, A.; Sárvári, É.; Solti, Á.; Czégény, G.; Hideg, É.; Hunyadi-Gulyás, É.; Bóka, K.; Böddi, B. Light Piping Driven Photosynthesis in the Soil: Low-Light Adapted Active Photosynthetic Apparatus in the under-Soil Hypocotyl Segments of Bean (Phaseolus Vulgaris). Journal of Photochemistry and Photobiology B: Biology 2016, 161, 422-429.

(9) Salisbury, F. J.; Hall, A.; Grierson, C. S.; Halliday, K. J. Phytochrome Coordinates Arabidopsis Shoot and Root Development. Plant J 2007, 50 (3), 429-438.

(10) Okon, Y. Azospirillum/Plant Associations; CRC Press, 1993.

(11) Okon, Y.; Vanderleyden, J. Root-Associated Azospirillum Species Can Stimulate Plants. ASM News 1997, 63 (7), 366-370.

(12) Steenhoudt, O.; Vanderleyden, J. Azospirillum, a Free-Living Nitrogen-Fixing Bacterium Closely Associated with Grasses: Genetic, Biochemical and Ecological Aspects. FEMS Microbiol. Rev. 2000, 24 (4), 487-506. 
(13) James, E.; Baldani, J. The Role of Biological Nitrogen Fixation by Non-Legumes in the Sustainable Production of Food and Biofuels. Plant and Soil 2012, 356, 1-3.

(14) Richardson, A.; Barea, J.; Mcneill, A.; Prigent-Combaret, C. Richardson AE, Barea JM, McNeill AM, Prigent-Combaret C.. Acquisition of Phosphorus and Nitrogen in the Rhizosphere and Plant Growth Promotion by Microorganisms. Plant Soil 321: 305-339. Plant and Soil 2009, 321.

(15) Y, O.; Ca, L.-G. Agronomic Applications of Azospirillum: An Evaluation of 20 Years Worldwide Field Inoculation. Soil Biol Biochem 1994, 26 (12), 1591-1601.

(16) Pedraza, R. O.; Bellone, C. H.; Carrizo de Bellone, S.; Boa Sorte, P. M. F.; Teixeira, K. R. dos S. Azospirillum Inoculation and Nitrogen Fertilization Effect on Grain Yield and on the Diversity of Endophytic Bacteria in the Phyllosphere of Rice Rainfed Crop. European Journal of Soil Biology 2009, 45 (1), 36-43.

(17) Dobbelaere, S.; Croonenborghs, A.; Thys, A.; Ptacek, D.; Vanderleyden, J.; Dutto, P.; Labandera-Gonzalez, C.; Caballero-Mellado, J.; Aguirre, J. F.; Kapulnik, Y.; Brener, S.; Burdman, S.; Kadouri, D.; Sarig, S.; Okon, Y. Responses of Agronomically Important Crops to Inoculation with Azospirillum. Functional Plant Biol. 2001, 28 (9), 871-879.

(18) Giraud, E.; Lavergne, J.; Verméglio, A. Chapter 9 - Characterization of Bacteriophytochromes from Photosynthetic Bacteria: Histidine Kinase Signaling Triggered by Light and Redox Sensing. In Methods in Enzymology; Methods in Enzymology: TwoComponent Signaling Systems, Part C; Academic Press, 2010; Vol. 471, pp 135-159.

(19) Westby, C. A.; Cutshall, D. S.; Vigil, G. V. Metabolism of Various Carbon Sources by Azospirillum Brasilense. Journal of Bacteriology 1983, 156 (3), 1369-1372.

(20) Goebel, E. M.; Krieg, N. R. Fructose Catabolism in Azospirillum Brasilense and Azospirillum Lipoferum. J Bacteriol 1984, 159 (1), 86-92.

(21) Alexandre, G.; Greer, S.; Zhulin, I. Energy Taxis Is the Dominant Behavior in Azospirillum Brasilense. Journal of bacteriology 2000, 182, 6042-6048.

(22) Pankievicz, V. C. S.; Amaral, F. P. do; Santos, K. F. D. N.; Agtuca, B.; Xu, Y.; Schueller, M. J.; Arisi, A. C. M.; Steffens, M. B. R.; Souza, E. M. de; Pedrosa, F. O.; Stacey, G.; Ferrieri, R. A. Robust Biological Nitrogen Fixation in a Model Grass-Bacterial Association. The Plant Journal 2015, 81 (6), 907-919.

(23) Braus-Stromeyer, S. A.; Schnappauf, G.; Braus, G. H.; Gössner, A. S.; Drake, H. L. Carbonic Anhydrase in Acetobacterium Woodii and Other Acetogenic Bacteria. J Bacteriol 1997, 179 (22), 7197-7200.

(24) Smith, K. S.; Ferry, J. G. A Plant-Type ( $\beta$-Class) Carbonic Anhydrase in the Thermophilic Methanoarchaeon Methanobacterium Thermoautotrophicum. Journal of Bacteriology 1999, 181 (20), 6247-6253. 
(25) Kaur, S.; Mishra, M. N.; Tripathi, A. K. Regulation of Expression and Biochemical Characterization of a Beta-Class Carbonic Anhydrase from the Plant Growth-Promoting Rhizobacterium, Azospirillum Brasilense Sp7. FEMS Microbiol Lett 2009, 299 (2), 149-158.

(26) Wenke, B. B.; Spatzal, T.; Rees, D. C. Site-Specific Oxidation State Assignments of the Iron Atoms in the [4Fe:4S]2+/1+/0 States of the Nitrogenase Fe-Protein. Angewandte Chemie International Edition 2019, 58 (12), 3894-3897.

(27) Zhang, Y.; Burris, R. H.; Ludden, P. W.; Roberts, G. P. Regulation of Nitrogen Fixation in Azospirillum Brasilense. FEMS Microbiol Lett 1997, 152 (2), 195-204.

(28) Cejudo, F. J.; Paneque, A. Short-Term Nitrate (Nitrite) Inhibition of Nitrogen Fixation in Azotobacter Chroococcum. Journal of Bacteriology 1986, 165 (1), 240-243.

(29) Carithers, R. P.; Yoch, D. C.; Arnon, D. I. Two Forms of Nitrogenase from the Photosynthetic Bacterium Rhodospirillum Rubrum. J Bacteriol 1979, 137 (2), 779-789.

(30) Sun, Q.; Yoda, K.; Suzuki, H. Internal Axial Light Conduction in the Stems and Roots of Herbaceous Plants. Journal of Experimental Botany 2005, 56 (409), 191-203.

(31) Housh, A. B.; Powell, G.; Scott, S.; Anstaett, A.; Gerheart, A.; Benoit, M.; Waller, S.; Powell, A.; Guthrie, J. M.; Higgins, B.; Wilder, S. L.; Schueller, M. J.; Ferrieri, R. A. Functional Mutants of Azospirillum Brasilense Elicit Beneficial Physiological and Metabolic Responses in Zea Mays Contributing to Increased Host Iron Assimilation. The ISME Journal 2021, 1-18.

(32) Inomura, K.; Deutsch, C.; Wilson, S. T.; Masuda, T.; Lawrenz, E.; Bučinská, L.; Sobotka, R.; Gauglitz, J. M.; Saito, M. A.; Prášil, O.; Follows, M. J. Quantifying Oxygen Management and Temperature and Light Dependencies of Nitrogen Fixation by Crocosphaera Watsonii. mSphere 2019, 4 (6).

(33) Fixen, K. R.; Zheng, Y.; Harris, D. F.; Shaw, S.; Yang, Z.-Y.; Dean, D. R.; Seefeldt, L. C.; Harwood, C. S. Light-Driven Carbon Dioxide Reduction to Methane by Nitrogenase in a Photosynthetic Bacterium. PNAS 2016, 113 (36), 10163-10167.

(34) Staley, C.; Ferrieri, A. P.; Tfaily, M. M.; Cui, Y.; Chu, R. K.; Wang, P.; Shaw, J. B.; Ansong, C. K.; Brewer, H.; Norbeck, A. D.; Markillie, M.; do Amaral, F.; Tuleski, T.; Pellizzaro, T.; Agtuca, B.; Ferrieri, R.; Tringe, S. G.; Paša-Tolić, L.; Stacey, G.; Sadowsky, M. J. Diurnal Cycling of Rhizosphere Bacterial Communities Is Associated with Shifts in Carbon Metabolism. Microbiome 2017, 5 (1), 65.

(35) Machado, H. B.; Funayama, S.; Rigo, L. U.; Pedrosa, F. O. Excretion of Ammonium by Azospirillum Brasilense Mutants Resistant to Ethylenediamine. Can. J. Microbiol. 1991, 37 (7), 549-553.

(36) Santos, A. R. S.; Etto, R. M.; Furmam, R. W.; Freitas, D. L. de; Santos, K. F. d'Eça N.; Souza, E. M. de; Pedrosa, F. de O.; Ayub, R. A.; Steffens, M. B. R.; Galvão, C. W. Labeled Azospirillum Brasilense Wild Type and Excretion-Ammonium Strains in Association with Barley Roots. Plant Physiology and Biochemistry 2017, 118, 422-426. 
(37) Näsvall, J.; Knöppel, A.; Andersson, D. I. Duplication-Insertion Recombineering: A Fast and Scar-Free Method for Efficient Transfer of Multiple Mutations in Bacteria. Nucleic Acids Res. 2017, 45 (5), e33.

(38) Pedrosa, F. O.; Yates, M. G. Regulation of Nitrogen Fixation (Nif) Genes of Azospirillum Brasilense by NifA and Ntr (Gln) Type Gene Products. FEMS Microbiology Letters 1984, 23 (1), 95-101. 


\section{Chapter Seven- Concluding Remarks: Global Concerns with Future Sustainable}

\section{Agriculture and Future Use of Radiotracers in the Real-World}

\subsection{A Recap of Challenges Facing Sustainable Agriculture}

In thirty years, the current global food production will be insufficient to feed the projected population of 9.1 billion people ${ }^{1}$. Maintenance of food security requires the global agricultural industry to increase production by $60 \%$ while combating water scarcity, climate change, and soil micronutrient deficiencies. Cereal grains like rice and corn are particularly important as they provide one-third of all food calories to more than 4.5 billion people living in 94 developing countries ${ }^{2}$. These grains are also vital as feed for the animal livestock portion of the world's food ${ }^{1}$. Progress in increased crop yield has been established by research networks via crop breeding methods, but increasing the quantity of food is not the only considerable issue ${ }^{3}$. In order to meet growing population demands agriculture needs to increase both quantity and quality crop products produced. Micronutrient deficiencies of the soil or crop product can lead to human undernutrition, especially in developing countries.

Iron deficiency is the most common nutritional deficiency in the world ${ }^{4}$. It is found in red blood cells for oxygen and carbon dioxide transport through the human body ${ }^{4}$. Additionally, deficiencies in iron and zinc have been identified as major underlying causes of multiple human diseases $^{5}$. In fact, according to the World Health Organization (WHO), $45 \%$ of all child deaths under five years of age are due to malnutrition ${ }^{5}$. Counteracting nutritional deficiency is necessary, but post-harvest biofortification is costly ${ }^{6}$. Pre-harvest in-field biofortification can be applied to improve crop micronutrient assimilation from the soil, transportation throughout the plant, and storage in seeds for later human consumption. Current solutions to crop micronutrient deficiency, such as supplemental fertilizers and genetic modification, are not easy to implement and can be difficult to garner public approval for. High-micronutrient fertilizers are financially 
burdensome to resource-poor farming communities and not enough is currently understood about the micronutrient needs of developing plants for this method to be efficient ${ }^{7}$. Genetic modification of cropping systems can be effective but is not generally accepted by the public. An alternative solution is needed.

\subsection{How Far We Have Come}

The application of radiotracers in plants began nearly 100 years ago in 1923, when George de Hevesy followed ${ }^{212} \mathrm{~Pb}$ absorption and allocation in horse-bean (Vicia faba) plants ${ }^{8}$. At the time, his administration technique of the radiometal to the plants was passive ${ }^{8}$, as we have done in our ${ }^{59} \mathrm{Fe}$ and ${ }^{65} \mathrm{Zn}$ work, and the uptake and allocation measurements were performed on separated tissues as we have also done. Modern radiation detection, such as $\mathrm{NaI}(\mathrm{Tl})$, are more easily utilized for dynamic tracer translocation measurements than de Hevesy's electroscope ${ }^{8}$ due to possible positioning and shielding of multiple detectors along the length of the plant stem to calculate transport speeds. Modern autoradiography also allows not only quantification of radioactivity in the various plant tissues, but also imaging of spatial patterning of the radioisotope within the tissue, adding more information. This measurement would not have been possible for de Hevesy as he ignited his plant tissues and measured the activity of their ash ${ }^{8}$.

Although this is solely a comparison between the earliest work with radiotracers in plants, the use of radiotracers is in this work is more dynamic, and includes combinations of radiation detection and imaging, and sometimes tracing more than one micronutrient within a project for the understanding of a problem or metabolic phenomenon. Here, radiotracers have been applied to understand micronutrient uptake and allocation patterns in maize for iron, zinc, and even boron. We have explored differences in uptake and allocation based on oxidation state of iron and have noted increases in its uptake and allocation to leaf tissue in the presence of plant growth promoting bacteria (PGPB) Azospirillum brasilense functional mutants including a 
promotion of ferrous iron uptake, which is not the natural strategy of graminaceous plants like maize, while confirming with autoradiography imaging showing difference spatial patterning in the shoots that the bacteria do not alter oxidation state of the radiotracer. The mechanisms of this iron promotion were explored using $\left[{ }^{11} \mathrm{C}\right] \mathrm{CO}_{2}$ to quantify metabolism of various metabolites and iron chelating molecules adding depth to the analysis. We were also able to determine leaf and root localization patterns of boron in maize based on a radiotracer designed to mimic a B binding in plantae using ${ }^{18} \mathrm{~F}$-labeled phenylboronic acid, which is the first time a radiotracer has been used to study boron nutrition in maize. While it is clear from these examples that radiotracing technologies have come a long way from their historic use, we still have a ways to go in implementing this technology in more "real-world" studies and applications.

\subsection{Rhizospheric Microorganisms as a Community- Not Isolates}

Increased interest in understanding plant-microbe interactions involving PGPB from the perspective of how they successfully colonize their host and influence its health has prompted extensive studies using cultures of microbial isolates, as seen in this dissertation work. While such interactions occur on minute spatiotemporal scales, the effects of PGPB can be more far-reaching, extending to the whole plant and spanning minutes-to-days over the host's life cycle. At the same time, the use of radionuclides, especially of short-lived positron emitting radionuclides, in fundamental plant-microbe research is rapidly expanding with increased availability of medical cyclotrons around the world and researchers in plant biology becoming trained in radiochemical skills and techniques.

This dissertation work has been completed on isolated strains of bacteria, but this is not representative of the environment in which crop plants actually exist. In fact, in recent years it has become obvious that the communities of microorganisms that occupy the rhizosphere surrounding 
the root-soil interface are complex ${ }^{9,10}$. We know little about the competitiveness of PGPB when inoculum isolates are administered into such communities. There are various analytical instruments and techniques, which allow insight into the complexity of these microbial community interactions with one another and the plant host such as integrated microscopy ${ }^{10}$, in which exchange of fluorescently labeled compounds in microbial communities can be measured live, as well as various 'omics techniques such as metabolomics, genomics, and proteomics- to name a few $^{10}$. There are some compounds of critical interest, which influence microbial colonization of their host plant and interactions with one another such as sugars, organic acids, amino acids, and phytohormones. Radiotracers would be perfectly suited for investigating microbial community responses to changes in these molecules because many are present in plants or as root exudates in very low concentrations where other analytical methods of detection might not be sensitive enough.

\subsection{Real-world Radiotracing}

Another aspect to "real-world" applications is growth in a field, in which there are complex conditions at play including rhizospheric microbial communities, varying soil characteristics and nutrient content, temperature, water availability, heat and salt stresses, pathogen exposure, and much more. The best way to begin understanding the complex relations involved in field growth of cropping system is to analyze plant growth and responses in outdoor field settings. One might think the use of radiotracers is a lab-only tool, but we can administer radiotracers to plant systems in a controlled setting allowing manipulation of the tracers and the plants for safety and better insight. In fact, this has already been done to some degree for the administration and detection of

$\left[{ }^{11} \mathrm{C}\right] \mathrm{CO}_{2}$ in an outdoor setting. A hand-held $\left[{ }^{11} \mathrm{C}\right] \mathrm{CO}_{2}$ delivery system was designed by Dohyun Kim, et al. in 2014 at Brookhaven National Laboratory in which $\left[{ }^{11} \mathrm{C}\right] \mathrm{CO}_{2}$ was trapped from the 
cyclotron production site on the portable device and transported to another site to administer to plants with $\sim 82 \%$ delivery efficiency ${ }^{11}$. This technology allows for the careful administration to live plants (and mature plants, which is often a limitation to examining large plants in traditional laboratory-based radiotracer studies) outdoors in a controlled manner. Ultimately, this allows detection or imaging of the administered radiotracer assuming portable technologies are adapted as well. Another study demonstrated the ability to adapt commercial PET scanners to quantitatively image ${ }^{11} \mathrm{C}$-assimilates in large plants- and was successful in measuring the allocation of these labeled assimilated from leaf tissue to roots still within soil without damaging or disturbing the plant system ${ }^{12}$. Such innovations have been accomplished for administration of $\left[{ }^{11} \mathrm{C}\right] \mathrm{CO}_{2}$ and PET imaging- there is much room for development of this technology into various other radiotracers and macro-/micronutrient tracing in living plants. The future of plant research in "realworld" radiotracing studies is already here and if we can take advantage of the technologies available and add innovation to create new and more useful tools, this could be a very valuable approach to overcoming many of our agricultural challenges to come. 


\section{References}

(1) Food security and why it matters https://www.weforum.org/agenda/2016/01/food-securityand-why-it-matters/ (accessed Mar 9, 2021).

(2) Shiferaw, B.; Prasanna, B. M.; Hellin, J.; Bänziger, M. Crops That Feed the World 6. Past Successes and Future Challenges to the Role Played by Maize in Global Food Security. Food Sec. 2011, 3 (3), 307.

(3) Cakmak, I. Plant Nutrition Research: Priorities to Meet Human Needs for Food in Sustainable Ways. Plant and Soil 2002, 247 (1), 3-24.

(4) Fanzo, J. Ethical Issues for Human Nutrition in the Context of Global Food Security and Sustainable Development. Global Food Security 2015, 7.

(5) Fact sheets - Malnutrition https://www.who.int/news-room/fact-sheets/detail/malnutrition (accessed Mar 9, 2021).

(6) Xue, Y.; Yue, S.; Zhang, W.; Liu, D.; Cui, Z.; Chen, X.; Ye, Y.; Zou, C. Zinc, Iron, Manganese and Copper Uptake Requirement in Response to Nitrogen Supply and the Increased Grain Yield of Summer Maize. PLOS ONE 2014, 9 (4), e93895.

(7) Hider, R. C.; Yoshimura, E.; Khodr, H.; Wirén, N. V. Competition or Complementation: The Iron-Chelating Abilities of Nicotianamine and Phytosiderophores. New Phytologist 2004, 164 (2), 204-208.

(8) Hevesy, G. The Absorption and Translocation of Lead by Plants. Biochem J 1923, 17 (4-5), 439-445.

(9) Staley, C.; Ferrieri, A. P.; Tfaily, M. M.; Cui, Y.; Chu, R. K.; Wang, P.; Shaw, J. B.; Ansong, C. K.; Brewer, H.; Norbeck, A. D.; Markillie, M.; do Amaral, F.; Tuleski, T.; Pellizzaro, T.; Agtuca, B.; Ferrieri, R.; Tringe, S. G.; Paša-Tolić, L.; Stacey, G.; Sadowsky, M. J. Diurnal Cycling of Rhizosphere Bacterial Communities Is Associated with Shifts in Carbon Metabolism. Microbiome 2017, 5 (1), 65.

(10) O’Banion, B. S.; O’Neal, L.; Alexandre, G.; Lebeis, S. L. Bridging the Gap Between Single-Strain and Community-Level Plant-Microbe Chemical Interactions. MPMI 2020, 33 (2), 124-134.

(11) Kim, D.; Alexoff, D. L.; Schueller, M.; Babst, B.; Ferrieri, R.; Fowler, J. S.; Schlyer, D. J. The Design and Performance of a Portable Handheld 11CO2 Delivery System. Applied Radiation and Isotopes 2014, 94, 338-343.

(12) Karve, A. A.; Alexoff, D.; Kim, D.; Schueller, M. J.; Ferrieri, R. A.; Babst, B. A. In Vivo Quantitative Imaging of Photoassimilate Transport Dynamics and Allocation in Large Plants Using a Commercial Positron Emission Tomography (PET) Scanner. BMC Plant Biol 2015, 15, 273. 


\section{Appendix: Correlating Bacterial Biological Function to Plant Carbon Allocation and Exudation}

A.1 Preface

This project was completed as a U.S. Department of Energy (DOE) Office of Science Graduate Student Research (SCGSR) award. That award program allows U.S. graduate students to complete thesis research in DOE national laboratories on research aligned with the DOE priority research areas. The study was carried out in two portions; 1) plant growth and inoculation with GFP-containing Herbaspirillum seropedicae (RAM10) as well as ${ }^{13} \mathrm{C}$ enrichment in labeling boxes at the University of Missouri Research Reactor (MURR) and 2) transport of the samples from the University of Missouri to Pacific Northwest National Lab (PNNL) where sampling and laser ablation isotope ratio mass spectrometry (LAIRMS) analysis was completed. Data was collected and compiled while a collaboration with a computational biologist will allow high quality quantitative and qualitative figures to be created for conclusions to be drawn. This appendix describes the work completed to date.

\section{A.2 Introduction}

Plant growth and productivity relies on nutrients available in the soil. Commonly, certain nutrients are not bioavailable in quantities for optimal growth and production. Nitrogen $(\mathrm{N})$ is a commonly limited macronutrient in soil systems and large amounts of $\mathrm{N}$ fertilizers are routinely applied in agriculture although they are often less than 50\% efficient. An alternative to enhance $\mathrm{N}$ bioavailability to plants is biological nitrogen fixation (BNF). Some plant growth promoting bacteria (PGPB) have been shown to provide $\mathrm{N}$ to plants and induce significant growth, but substantial knowledge gaps remain regarding the mechanisms of these interactions. Many BNF PGPB naturally associate with plant root systems, are found in high numbers $\left(10^{8} \mathrm{cfu} / \mathrm{gfw}\right)$, and 
yet do not induce noticeable plant stress responses ${ }^{1}$. Of particular interest to grass species is the endophyte Herbaspirillum seropedicae (SMR1), which has been shown to provide $\mathrm{N}$ to the host plant as well as enhance growth $(\text { S. veridis })^{2}$, iron assimilation, and iron allocation to shoot tissue (Z. mays $)^{3}$. These studies demonstrate SMR1 can have significant effects on the plant metabolic processes- including carbon $(\mathrm{C})$ allocation and exudation.

Plants provide ample organic $\mathrm{C}$ to the rhizosphere through root exudation, which can stimulate microbial activity. As plants provide $\mathrm{C}$ to the soil, microbes provide nutrients to the plant in a dynamic exchange resulting from complex, multi-kingdom interactions. These interactions exist heterogeneously in space and time as pockets of greater exudation and microbial activity are related to factors such as soil characteristics, root growth, and root differentiation. Spatial investigation, based on areas of known root association with SMR1, can be accomplished with a stable isotope tracer $\left({ }^{13} \mathrm{CO}_{2}\right)$ using Laser Ablation Isotope Ratio Mass Spectrometry (LA-IRMS) ${ }^{4}$. These methods allows for spatial selection across the rhizosphere, ablation of the particulates along the laser track, and analysis of changes in isotopic ratios. Tracking plant-derived $\mathrm{C}$ through the rhizosphere to microbial consumers, such as SMR1, will provide information about this complex nutrient exchange, better understanding how this particular PGPB can benefit the growth and bioenergy characteristics of Panicum virgatum (switchgrass).

\section{A.3 Materials and Methods}

GFP-reporting mutant strain of SMR1 (RAM10) Herbaspirillum seropedicae was grown in liquid NFbHP-malate medium following published procedures ${ }^{2}$. The medium contains $20 \mathrm{mM}$ ammonium chloride as a nitrogen source and streptomycin antibiotic $\left(80 \mu \mathrm{gL}^{-1}\right)$. The cultures were grown in a shaking incubator at $30^{\circ} \mathrm{C}$ and $130 \mathrm{rpm}$ for 24 hours before harvest and 
inoculation. Switchgrass seeds (Panicum virgatum L. var. Cave-in-rock; USDA-NRCS, 2011) were obtained from the Environmental and Molecular Sciences Laboratory (EMSL) at PNNL and germinated at the University of Missouri Research Reactor (MURR) by placing an Eppendorf containing seeds in a $-80^{\circ} \mathrm{C}$ freezer for 24 hours followed by placement of the seeds individually into $15-\mathrm{mL}$ falcon tubes containing gel growth media. The gel growth media was prepared in $500 \mathrm{~mL}$ bulk and aliquots were made into sterile falcon tubes while hot and liquid. The media contained $500 \mathrm{~mL}$ deionized (DI) water, $1.75 \mathrm{~g}$ Gel-Rite, $0.813 \mathrm{~g}$ of Hoagland's, $0.275 \mathrm{~g}$ MES, and was $\mathrm{pH}$ adjusted to 6.00 with $30 \% \mathrm{KOH}$. The seedlings were inoculated as appropriate to treatment type with the RAM10 culture, consisting of adding a washed and condensed $1 \mathrm{~mL}$ bacteria pellet to a petri dish of $\sim 10$ seeds and rocking in the shaking incubator

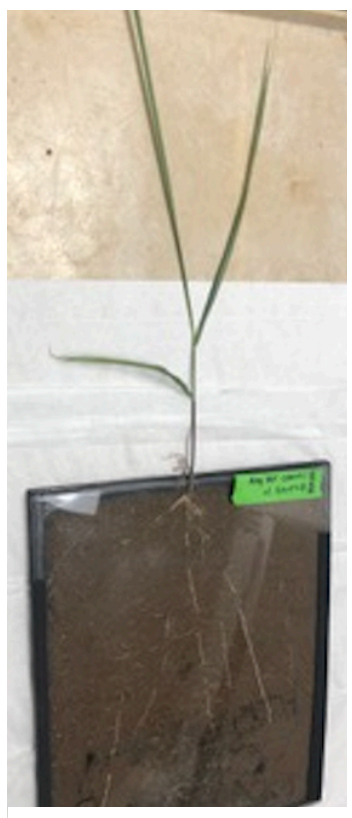

Figure A.3.1. Switchgrass in rhizobox setup. for two hours before seedling placement into plastic soil-containing rhizoboxes (Figure A.3.1). The soil (sandy loam Alfisol) used in this study was collected from 0-30 cm depth from plots in the W.K. Kellogg Biological Station associated with Michigan State University located in Hickory Corners, Michigan, USA. These soil plots were under continuous switchgrass cultivation and provide an authentic switchgrass microbiome. Before packing the rhizoboxes with soil, it was sifted through a fine mesh to support uniform packing into the thin rhizoboxes. Rhizoboxes were clear 8 " by 11 " inch plastic on the front face and black plastic on the back portion. The rhizobox had a small hole cut out for the shoots to grow outside of the box, and the front and back portions of the box were sealed 
and held tightly together by multiple clips

(Figure A.3.1.). Once assembled with plant inside, the rhizoboxes were wrapped in aluminum foil to shield the roots from light exposure.

Growth occurred in indoor growth chambers for approximately 10 weeks in the rhizoboxes (slanted at an approximate $45^{\circ}$ angle so root would grow against clear front plate) prior to exposure to labeled ${ }^{13} \mathrm{CO}_{2}$. Nutrient and water were provided via a paper towel inserted through the bottom of the rhizobox wicking up moisture. Water was constantly available and Hoagland's growth nutrient was administered through the top where the shoots emerged via syringe every 3 days. Growth conditions consisted of 12-hour photoperiods, $500 \mu \mathrm{mol} \mathrm{m}^{-2} \mathrm{~s}^{-1}$ light intensity, and

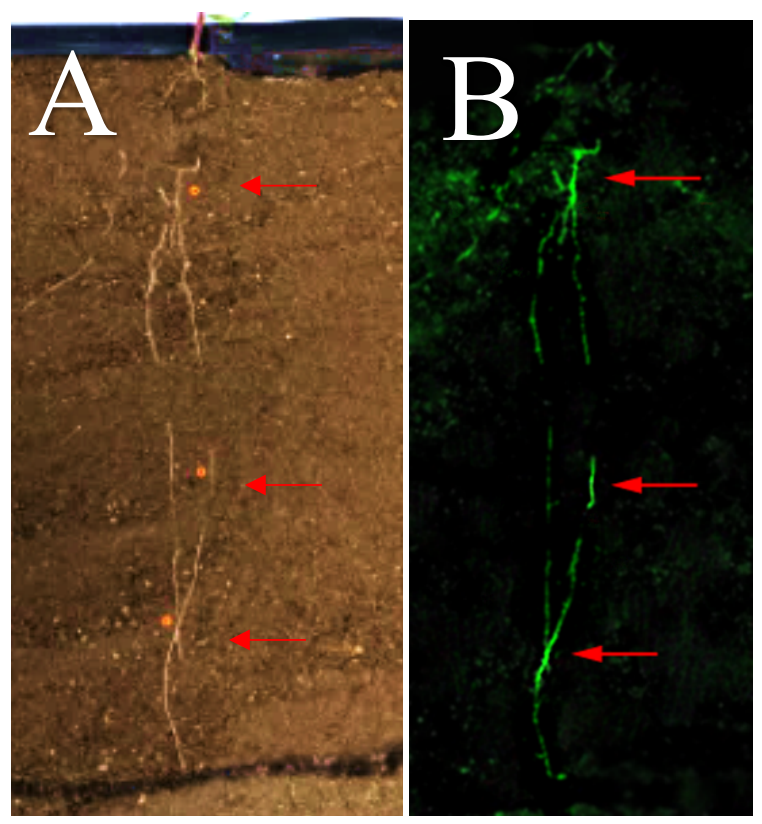

Figure A.3.2. Localization of high colonization of roots by RAM10 $\mathrm{H}$. seropedicae by fluorescence imaging. The localization of root locations with greater colonization of PGPB, noted by high fluorescence (Panel B), allowed marking zones of interest for laser ablation studies as well as locations near which to place $\left({ }^{15} \mathrm{NH}_{4}\right)_{3} \mathrm{PO}_{4}$ spots (not pictured here). (Panel A) Locations of high bacteria colonization were marked with orange beads (here shown in red circles) while locations of added nutrient patch were marked with white beads. temperatures of $25^{\circ} \mathrm{C} / 20^{\circ} \mathrm{C}$ (light/dark) with humidity at $70-80 \%$. There were four treatment types ( $\mathrm{n}=3$ each, 12 plants total): non-inoculated control switchgrass in Kellogg soil, RAM10 inoculated switchgrass in Kellogg soil, non-inoculated control switchgrass in Kellogg soil with an enriched $\left({ }^{15} \mathrm{NH}_{4}\right)_{3} \mathrm{PO}_{4}$ chemical spot treatment, and RAM10 inoculated switchgrass in Kellogg soil with the enriched $\left({ }^{15} \mathrm{NH}_{4}\right)_{3} \mathrm{PO}_{4}$ chemical spot treatment. 
Plant propagation, bacteria inoculation, and fluorescence localization of the bacteria in the roots with a Typhoon 9000 imager was completed at MURR prior to full-time research at PNNL. This imaging (fluorescence detection with a $473 \mathrm{~nm}$ blue incidence laser and green fluorescence filter) confirmed bacterial inoculation and aided in specifying locations for placement of the chemical spot treatment and later laser ablation in inoculated plant treatments (Figure A.3.2.).

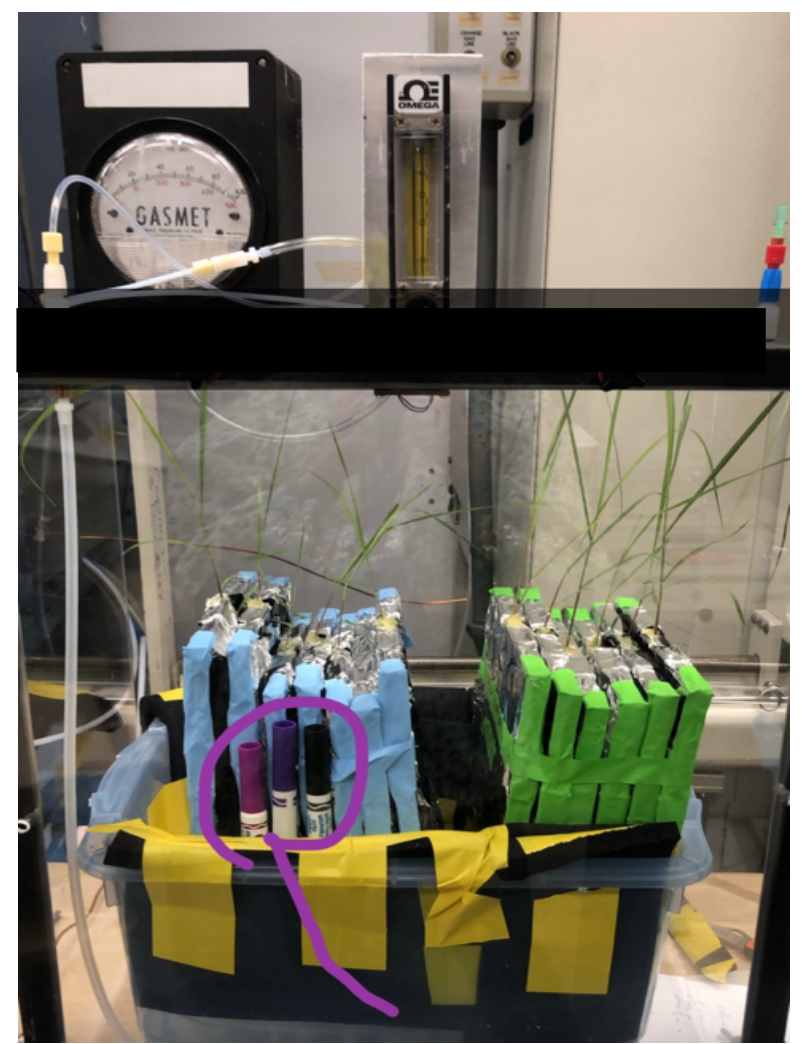

Figure A.3.3. ${ }^{13} \mathrm{CO}_{2}$ incubation chamber setup for switchgrass plants. Both inoculated and non-inoculated plants were placed within the same plexiglass, airtight incubation chamber as described. The noninoculated treatment plants are pictured in blue, while inoculated treatment plants are in green. Plants wicked up water constantly from a paper towel immersed in a basin of water and enclosed inside the bottom of the rhizobox. Chance of cross contamination was minimized by using two separate water basins; one per treatment type.
Once a preferable location is determined close to any roots of interest, two separate 50 $\mu \mathrm{L}(20 \mu \mathrm{M})\left({ }^{15} \mathrm{NH}_{4}\right)_{3} \mathrm{PO}_{4}$ aliquots were applied as needed by pipet to soil near the roots. Plants were returned to their growth chamber but placed within a gas-tight stable isotope labeling chamber to grow exposed to ${ }^{13} \mathrm{CO}_{2}$ for a week before plant harvesting occurred (Figure A.3.3.).

Stable isotope treatment was delivered directly into the gas-tight chamber from the zero air and ${ }^{13} \mathrm{CO}_{2}$ reference gas $(99.8 \%$ enriched) cylinders with a circulation fan within the chamber to maintain even distribution of the labeled carbon dioxide to a level of $400 \mathrm{ppm}$ in the air. This was confirmed daily by removal of chamber air and measurement of $\mathrm{CO}_{2}$ on the gas 
chromatograph (GC). The GC method included a flame ionization detector (FID) for quantification of $\mathrm{CO}_{2}$ as methane. $\mathrm{CO}_{2}$ was reduced on line to methane using reduced nickel catalyst at $400^{\circ} \mathrm{C}$. The column used was a packed column and the temperature gradient of the method began at $40^{\circ} \mathrm{C}$ with a two-minute hold and then increased $10^{\circ} \mathrm{C}$ min$^{-1}$ until reaching the final temperature of $200^{\circ} \mathrm{C}$. The peak of interest occurred at approximately 8.48 minutes. Following a one-week incubation with $\left[{ }^{13} \mathrm{C}\right] \mathrm{CO}_{2}$, the shoots were removed and rhizoboxes were dried in an oven to kill existing microbes and preserve samples for shipping to EMSL in Richland, WA.

For the investigation of how SMR1 association and biological function affects carbon allocation and exudation in switchgrass, $\left({ }^{13} \mathrm{C} /{ }^{12} \mathrm{C}\right)$ was measured in the root, immediate rhizosphere and across the soil to the $\left({ }^{15} \mathrm{NH}_{4}\right)_{3} \mathrm{PO}_{4}$ chemical nutrient spot (as appropriate) to infer plant trafficking of carbon sources. To accomplish this, cylindrical samples were punched out of the soil matrices (Figure A.3.4. A\&B) for analysis in the laser ablation isotope ratio mass spectrometer (LAIRMS) (Sercon Innovators in Isotopes 20-22 Stable Isotope Ratio Mass Spectrometer, Crewe, U.K.). This was completed with a copper punch placed over the soil location of interest (approximately $1.4 \mathrm{~cm}$ diameter) which was twisted gently into soil until reaching the back plate. A scalpel was used to cut roots away from the rhizobox along the edges of the punch. Two drops of DI water were added to the dry soil area within the copper punch and a plastic press was used to compress the sample into a firm, uniform puck shape. Lifting the copper tool allowed the soil sample to lift cleanly from the rhizobox and it was transferred to a sheet of aluminum foil for storage within a plastic petri dish for hydraulic pressing (Figure A.3.4 B). 
The samples were frozen in a $-80{ }^{\circ} \mathrm{C}$ freezer overnight before being hydraulically pressed on top of free soil from the same rhizobox the sample was from. This was done to enhance the stability of the thin soil samples while manually manipulated during sample loading into the laser chamber. Once pressed, the samples were photographed under a microscope (Olympus Corporation stereomicroscope with Highlight 3000 fiber optic light source illuminator, Massachusetts, USA) for ablation mapping (Figure A.3.4. C). Prior to loading the soil samples into the laser chamber, a piece of fishing line (Zebco Omniflex $12 \mathrm{lb}$ fishing line, Oklahoma, USA) was cut to approximately $0.5 \mathrm{~cm}$ length and stuck to the surface of the sample with a piece of double-sided tape of appropriate size. The fishing line served as a non-enriched carbon standard and was placed out of the way of the root(s) on the surface on the soil sample.
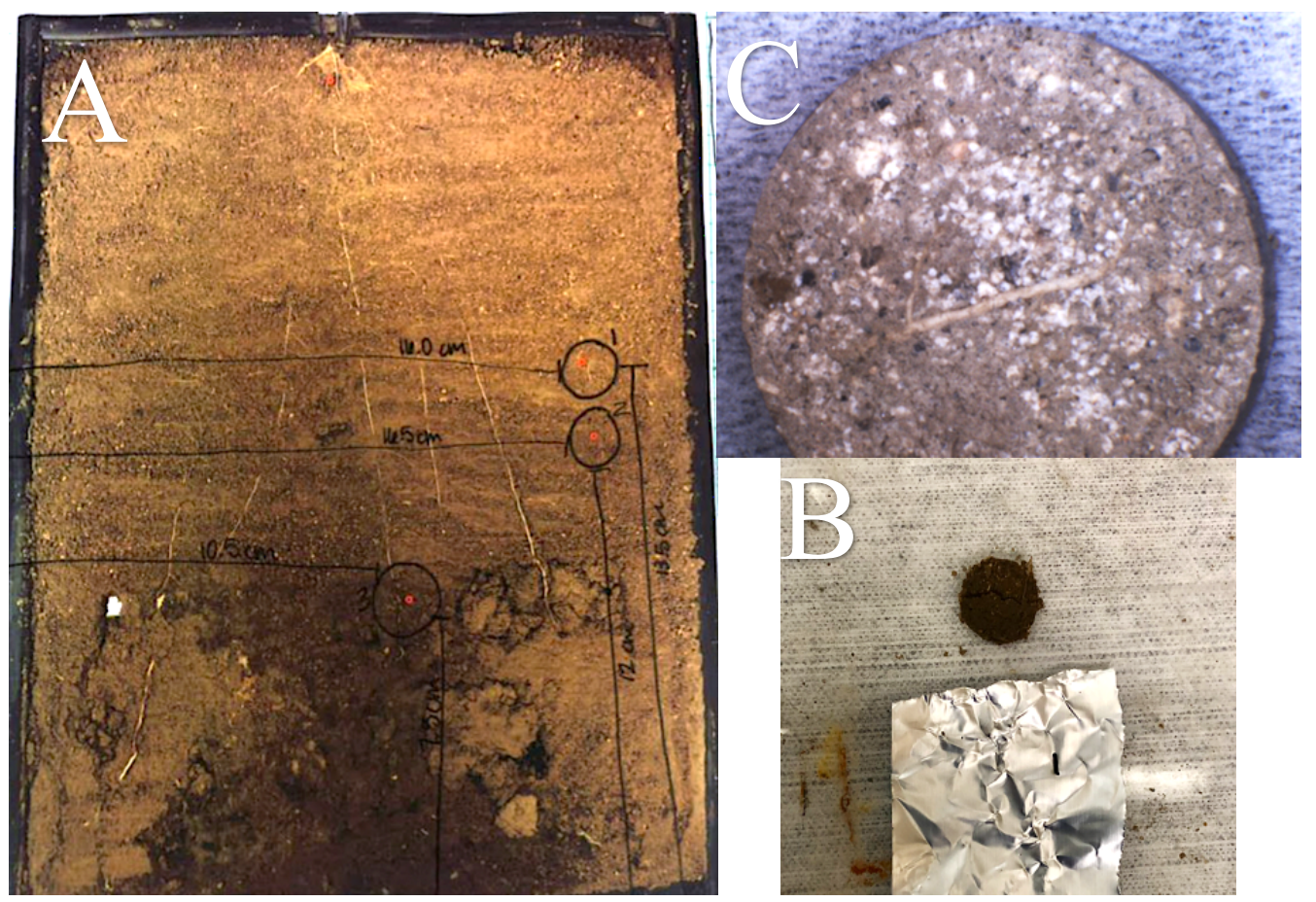

Figure A.3.4. Sample preparation for LAIRMS analysis. Panel A: Rhizoboxes were sampled based on root samples indicating high bacteria localization (as appropriate) and the samples taken were marked and mapped by distance from the rhizobox edges (in $\mathrm{cm}$ ). Panel B: Upon pressing and removal of the soil sample from the rhizobox, the sample was placed on marked $\mathrm{Al}$ foil for storage until ready for freezing at $-80{ }^{\circ} \mathrm{C}$ freezer and subsequent hydraulic pressing. Panel C: After pressing, samples were photographed under a light microscope to aid in ablation mapping of the sample. 
For each individual soil sample ablated, the fishing line standard was ablated first and last on a day where sample analysis was completed to set a background. In general, each ablation track had 7-9 individual ablations with laser settings at $80 \%$ power for fishing line, and $100 \%$ power for all soil ablations. The laser frequency was set to $20 \mathrm{~Hz}$ with a $50 \mu \mathrm{m}$ diameter per ablation for all ablations. The first ablation track was always completed on a space of soil set as far from the root as possible, and subsequent ablation tracks were completed from near the root $(\sim 250 \mu \mathrm{m}$ from the root edge) and away from the root, with $\sim 400 \mu \mathrm{m}$ between individual ablations. Generally, between ablation tracks there was $1200 \mu \mathrm{m}$ spacing. Ablations of the root tissue were always completed last and followed the shape of the root keeping $700 \mu \mathrm{m}$ between each ablation spot. Fishing line ablations generally required 8 laser shots, soil ablations ranged between 200440 shots, and root ablations required 10 shots. Coordinates (X, Y) were recorded for each ablation spot for figure formation as they relate to three fiducial markings on each sample. For each sample ablated, the image of the pressed sample prior to ablation was used to manually map the individual ablation tracks (Figure A.3.5 A). For ultimate data analysis and production of figures, root masking was completed in which the root within the sample photograph was filled in with white to make it easily distinguishable from non-root portions of the image (Figure A.3.5 B). Then, measurements of the distance from manual mapped ablation track distances to the center of the root were measured in pixels and scaled to sample diameter $(1.4 \mathrm{~cm})$ (Figure A.3.5. C).

The ${ }^{13} \mathrm{C} /{ }^{12} \mathrm{C}$ ratios measured in the soil of the samples will be normalized to the ${ }^{13} \mathrm{C} /{ }^{12} \mathrm{C}$ values measured in the root tissues and this value will be plotted by distance from root to determine if there is an appreciable difference in root exudation patterns between the treatment 
types analyzed. Additionally, isotope ratio heat mapping will be completed in order to visualize these patterns.

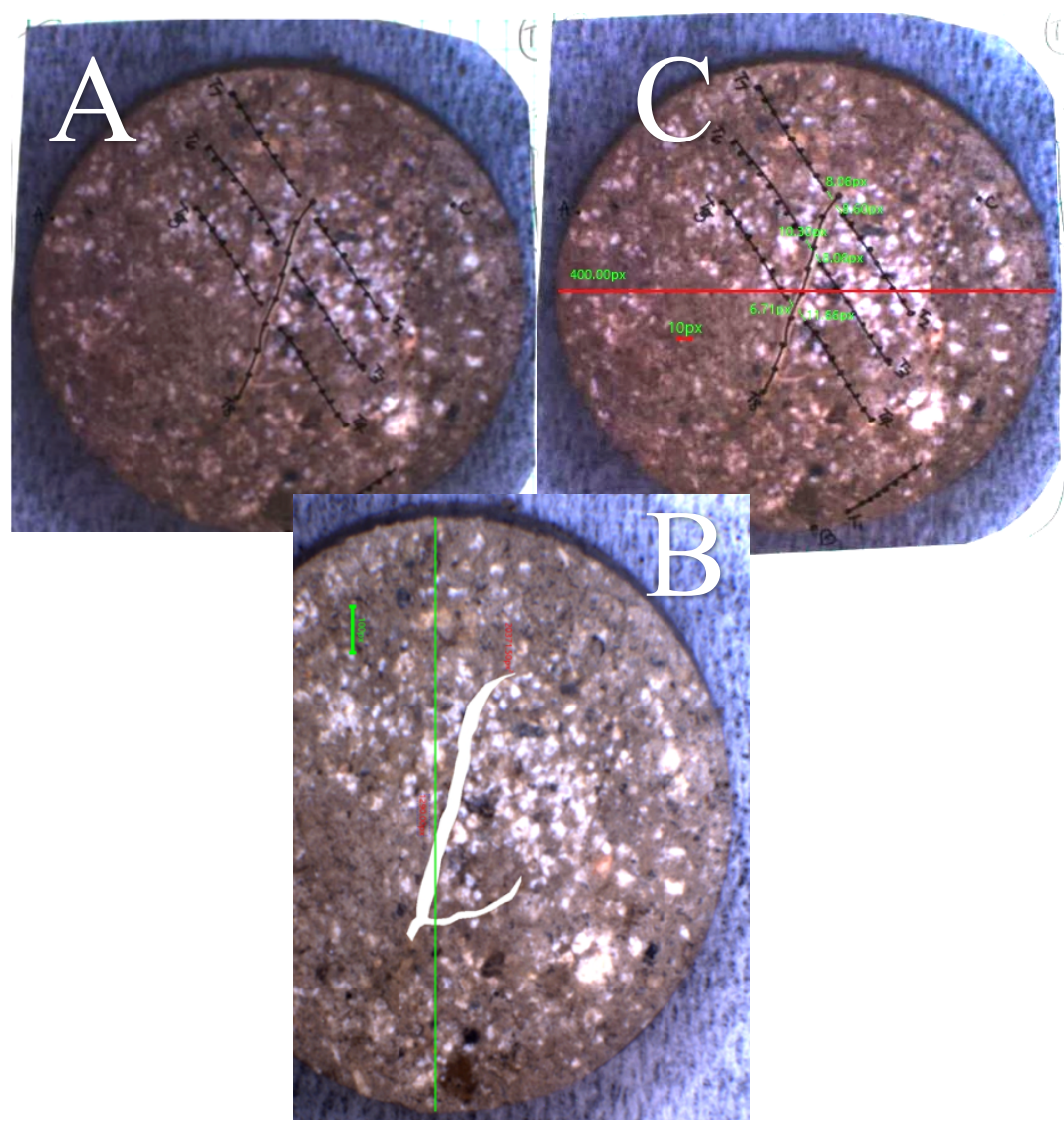

Figure A.3.5. Root mapping of ablations and data collection for analysis. Panel A: Manual mapping of the individual ablations (dots) connected through ablation tracks (lines connecting dots). Panel B: Root masked in white shape fill completed on AmScope microscope camera software. Here the image has been rotated to match the orientation of the sample in Panels A\&C. Distances were scaled in green and reference bars were added. Panel C: Measurements were completed in pixels on the AmScope software from the closest edge of the ablation track to the center of the root. The distances measured appear on the image in green. The image was scaled (in red) and allows relation of pixels to physical distance (diameter of the sample in $\mathrm{cm}$ ).

\section{A.4 Results}

This project is located in the appendix due to pending data analysis. Due to personnel transfer in and out of Pacific Northwest National Laboratory, collaboration on the results of the study was delayed. The Isotope Analysis Lab within the Environmental and Molecular Science Laboratory building at Pacific Northwest National Laboratory provides unique stable isotope technology needed to answer our research questions. Spatial distribution of carbon isotope ratios as ${ }^{13} \mathrm{CO}_{2}$ will be used to understand the influences of RAM10 on carbon utilization, allocation to roots, and exudation in switchgrass. Laser Ablation Isotope Ratio Mass 
Spectrometry (LAIRMS) allows ablation of soil particulates along the laser track, quantification of carbon isotopic ratios, and will provide information about the C-13 enrichment patterns of roots and immediate rhizosphere under non-inoculated and RAM10 inoculated conditions, as well as under circumstances of elevated soil ammonium content. 


\section{References}

(1) Agtuca, B.; Stopka, S. A.; Tuleski, T. R.; Amaral, F. P. do; Evans, S.; Liu, Y.; Xu, D.; Adele Monteiro, R.; Koppenaal, D. W.; Paša-Tolić, L.; Anderton, C. R.; Vertes, A.; Stacey, G. In Situ Metabolomic Analysis of Setaria Viridis Roots Colonized by Beneficial Endophytic Bacteria. Mol. Plant Microbe Interact. 2019.

(2) Pankievicz, V. C. S.; do Amaral, F. P.; Santos, K. F. D. N.; Agtuca, B.; Xu, Y.; Schueller, M. J.; Arisi, A. C. M.; Steffens, M. B. R.; de Souza, E. M.; Pedrosa, F. O.; Stacey, G.; Ferrieri, R. A. Robust Biological Nitrogen Fixation in a Model Grass-Bacterial Association. Plant J 2015, 81 (6), 907-919.

(3) Housh, A. B.; Powell, G.; Scott, S.; Anstaett, A.; Gerheart, A.; Benoit, M.; Waller, S.; Powell, A.; Guthrie, J. M.; Higgins, B.; Wilder, S. L.; Schueller, M. J.; Ferrieri, R. A. Functional Mutants of Azospirillum Brasilense Elicit Beneficial Physiological and Metabolic Responses in Zea Mays Contributing to Increased Host Iron Assimilation. The ISME Journal 2021, 1-18.

(4) Denis, E. H.; Ilhardt, P. D.; Tucker, A. E.; Huggett, N. L.; Rosnow, J. J.; Moran, J. J. Spatially Tracking Carbon through the Root-Rhizosphere-Soil System Using Laser AblationIRMS. Journal of Plant Nutrition and Soil Science 2019, 182 (3), 401-410. 


\section{VITA}

Alexandra B. Housh was born and raised in northeastern Ohio. She later moved to Tiffin, Ohio where she earned a bachelor's degree in chemistry and biology from Heidelberg University in 2017. During her undergraduate experience, she completed research on analytical methods for water quality investigation and participated in the Nuclear Chemistry Summer School program in California, sponsored by the Department of Energy, where she gained a keen interest in research, especially in the field of radiochemistry. In the fall of 2017, Alexandra joined the University of Missouri's Department of Chemistry and Dr. Ferrieri's research group. As a graduate student, she gained knowledge of radio-analytical and traditional analytical techniques, including ${ }^{18} \mathrm{~F}$ radiotracer synthesis, ICP-MS, LA-ICP-MS, radio-HPLC, ion chromatography, phosphor imaging, and various other analytical techniques applying them toward investigating the biological functions of plants and plant-microbial interactions. She obtained her Ph.D. in 2021, after which she took a position as a National Nuclear Security Administration Fellow in Washington, DC. 